\title{
Doppler ultrasound tests in the diagnosis of chronic aortoiliac obstruction
}

Citation for published version (APA):

Kitslaar, P. J. E. H. M. (1982). Doppler ultrasound tests in the diagnosis of chronic aortoiliac obstruction. [Doctoral Thesis, Maastricht University]. Uitgeversmaatschappij Huisartsenpers B.V. https://doi.org/10.26481/dis.19820326pk

Document status and date:

Published: 01/01/1982

DOI:

10.26481/dis.19820326pk

Document Version:

Publisher's PDF, also known as Version of record

\section{Please check the document version of this publication:}

- A submitted manuscript is the version of the article upon submission and before peer-review. There can be important differences between the submitted version and the official published version of record.

People interested in the research are advised to contact the author for the final version of the publication, or visit the DOI to the publisher's website.

- The final author version and the galley proof are versions of the publication after peer review.

- The final published version features the final layout of the paper including the volume, issue and page numbers.

Link to publication

\footnotetext{
General rights rights.

- You may freely distribute the URL identifying the publication in the public portal. please follow below link for the End User Agreement:

www.umlib.nl/taverne-license

Take down policy

If you believe that this document breaches copyright please contact us at:

repository@maastrichtuniversity.nl

providing details and we will investigate your claim.
}

Copyright and moral rights for the publications made accessible in the public portal are retained by the authors and/or other copyright owners and it is a condition of accessing publications that users recognise and abide by the legal requirements associated with these

- Users may download and print one copy of any publication from the public portal for the purpose of private study or research.

- You may not further distribute the material or use it for any profit-making activity or commercial gain

If the publication is distributed under the terms of Article $25 \mathrm{fa}$ of the Dutch Copyright Act, indicated by the "Taverne" license above, 
Doppler ultrasound tests

in the diagnosis of

chronic aortoiliac obstruction 
The study reported in this thesis was subsidiced by the Netherlands Heart Foundation (Grant-in-aild 79,004).

The publication of the thesis was financially supported by the Netherlands Heart Foundation and the St. Antonius Hospital in Utrecht. 


\section{Doppler ultrasound tests in the diagnosis of chronic aortoiliac obstruction}

\section{PROEFSCHRIFT}

Ter verkrijging van de graad van doctor in de geneeskunde aan de Rijksuniversiteit Limburg te Maastricht op gezag van de rector magnificus Prof. Dr. H.C. Hemker, volgens besluit van het College van Dekanen in het openbaar te verdedigen in de aula van de universiteit op vrijdag 26 mart 1982 des namiddags te vier uur

door

PETRUS JOHANNES EVERARDUS HENRICUS MARIA KITSLAAR geboren te Roermond 
Promotores: $\quad$ Prof. Dr. R.S. Reneman

Prof. Dr. Ir. J.E.W. Beneken

Referenten: Prof. Dr. Ir. N. Bom

Prof. Dr. J.M.F. Mol

Prof. Dr. J.L. Terpstra 
To all those who taught me surgery 
"The fundamental problems in the circulation derive from that fact that the supply of adequate amounts of blood to the organs of the body is the main purpose of the circulation and the pressures that are necessary to achieve it are of secondary importance; but the measurement of flow is difficult while that of pressure is easy so that our knowledge of flow is usually derivatory."

\section{D.A. MCDONALD, 1974, quoting his teacher Prof. K. FRANKLIN}

"There are few areas in medicine in which the conditions encountered lend themselves so readily to diagnosis solely on the basis of thoughtful history and careful physical examination as do vascular diseases." 


\section{PREFACE}

This thesis is the result of a study which I performed along with my daily work in the department of surgery of the St. Antonius Hospital in Utrecht. It, therefore, could only be completed thanks to the sympathetic attitude of staff and colleagues of that department towards my efforts. I gratefully acknowledge the special support of the senior vascular surgeon F.E.E. Vermeulen, M.D., who was always ready for discussion and who actively participated in the study by reviewing the angiograms in his characteristic meticulous way.

The very nature of this study entailed a considerable contribution of engineers and non-medical technicians to it. This, in turn, gave me the opportunity to pursue activities outside my own occupation in their specific fields of interest. This was an exiting and fruitful experience. It taught me a lot about the potential of computers and other electronic devices in the medical field and something about their limitations.

I owe a great deal to A.P.G. Hoeks, M.Sc., and C.J. Ruissen for introducing me in Doppler technology and audio frequency spectrum analysis and for the hospitality, the facilities and the assistance they gave me in their ultrasound laboratory at the University of Maastricht.

It is not too much to say that the most important contribution to this study certainly was made by J.L. Talmon, M.Sc.. He developed the various computer programs for signal analysis and data processing. He also performed all computerized analyses, including the statistical ones, often continuing that work far into the late evening hours.

Finally, I wish to thank the following persons which have been of great assistance to me in different stages of the study: Mr. S. van den Molen who built or modified the electronic equipment for this study, Mr. R. van Staden who made my escapades on the computer possible and Mrs. A. Blok-Stronkhorst who did all the secretarial work and type-writing for this thesis. 


\section{CONTENTS}

1. INTRODUCTION 13

1.1. Reason to perform the study

1.2. Aim of the study 14

1.3. Contents and division of the thesis 15

2. CHRONIC AORTOILIAC OBLITERATIVE DISEASE 17

2.1. General aspects of chronic aortoiliac obliterative disease $\quad 17$

$\begin{array}{ll}\text { 2.1.1. Terminology and definitions } & 17\end{array}$

2.1.2. Pathology and etiology 17

2.1.3. Anatomic patterns of the disease 17

2.1.4. Occurrence and frequency distribution of aortoiliac

2.2. The diagnosis of chronic aortoiliac obstruction 19

$\begin{array}{ll}\text { 2.2.1. Symptoms and signs } & 20\end{array}$

2.2.2. Angiography 2]

2.2.3. Indirect blood pressure measurements 22

2.2.4. Direct arterial blood pressure measurements 22

$\begin{array}{ll}\text { 2.2.5. Pulse waveform analysis } & 23\end{array}$

2.2.6. Blood flow measurements 24

2.2.7. Measurement of aorto-femoral transit times 25

3. HEMODYNAMIC AND PATHOPHYSIOLOGIC ASPECTS OF THE ARTERIAL CIRCULATION OF THE LIMBS 27

3.1. Basic principles $\quad 27$

3.2. Normal pulsatile arterial blood pressures 28

3.3. Normal pulsatile arterial velocities $\quad 29$

3.3.1. The relation between pulsatile arterial pressure and flow $\quad 29$

$\begin{array}{ll}3.3 .2 \text {. Flow velocity pulse wave } & 30\end{array}$

3.3.3. Flow velocity profiles 30

3.3.4. Reverse flow $\quad 32$

3.4. Hemodynamic resistance and impedance 33

3.5. Influences of vessel geometry on pulsatile pressure and flow
complexes

3.5.1. Normal vessel geometry 34

3.5.2. Stenosis 34

3.5.2.1. Fluid energy losses due to stenosis 34

3.5.2.2. Critical stenosis, length of stenosis and multiple
stenoses

3.5.2.3. Pressure and flow pulse wave changes due to
stenosis

3.5.3. Aneurysms 38

4. ULTRASONIC DOPPLER BLOOD FLOW VELOCIMETRY 39

4.1. Principles of ultrasonic Doppler flow velocity detection 39

4.2. Doppler blood flow velocity detectors 41 
4.2.1. Continuous wave (CW) Doppler systems 41

4.2.2. Pulsed Doppler (PD) meters $\quad 42$

4.2.3. Directional information $\quad 42$

4.3. The Doppler frequency spectrum

4.4. Factors influencing the Doppler frequency spectrum

4.5. Methods of Doppler audiosignal processing 44

4.5.1. Acoustic presentation $\quad 45$

4.5.2. Zero Crossing Detection (ZCD) 45

4.5.3. Audio frequency spectrum analysis $\quad 46$

5. SEGMENTAL SYSTOLIC PRESSURE MEASUREMENTS IN THE LEGS

5.1. Methods of measurements 49

5.2. The use of hyperemia in pressure measurements 50

5.3. Normal pressures at rest and during hyperemia 52

5.4. Correlation with the clinical stage of the disease 52

5.5. Relation between segmental pressures and angiographic findings

5.5.1. Discrimination between limbs with obstructions and limbs without obstruction at angiography

5.5.2. Differentiation between single level and multiple level angiographic obstructions

5.5.3. Segmental pressures with well-defined angiographic obstructions

5.5.4. Anatomic diagnosis of arterial obstructions by segmental pressure measurements in the leg.

5.6. Conclusions

6. ANALYSIS OF DOPPLER SIGNALS OF THE ARTERIES OF THE LEG

6.1. Introduction 59

6.2. Qualitative signal analysis $\quad 59$

6.2.1. Acoustic classification $\quad 59$

6.2.2. Classifications by pattern recognition 60

$\begin{array}{ll}\text { 6.3. Quantitative signal analysis } & 62\end{array}$

6.3.1. Signal amplitude parameters $\quad 63$

6.3.2. Time-related parameters 65

6.3.3. Pulsatility index 65

6.3.4. New developments in Doppler signal analysis 69

7. SEGMENTAL DOPPLER PARAMETERS OF THE LOWER EXTREMITIES

$\begin{array}{lr}\text { 7.1. Introduction } & 71 \\ \text { 7.2. Transit time measurement } & 72\end{array}$

7.3. Damping factor 73

7.4. Combined use of transit time and damping factor 74

8. THE EVALUATION OF THE AORTOILLAC PATHWAY BY SEGMENTAL SYSTOLIC PRESSURE MEASUREMENT, DOPPLER SIGNAL ANALYSIS AND SEGMENTAL DOPPLER PARAMETER DETERMINATION 
8.1. Segmental pressure measurement 77

8.2. Doppler signal analysis 78

8.3. Segmental Doppler parameters 79

8.4. Combined use of pressure and Doppler signal or segmental Doppler parameters

8.5. Conclusions

9. METHODS.

EXAMINATIONS, MATERIAL, INSTRUMENTATION AND DATA ANALYSIS

9.1. General set-up of the study 83

9.2. Selected noninvasive ultrasonic Doppler tests 83

$\begin{array}{lr}\text { 9.3. Population studied } & 84\end{array}$

9.4. Equipment and instruments $\quad 87$

9.4.1. Doppler signal registration system $\quad 87$

9.4.2. Doppler signal display system 88

9.4.3. Channel separation of the Doppler system 89

9.4.4. Sphygmomanometric equipment 90

9.5. Examination protocol and description of tests 90

9.6. Angiography 92

9.7. Data processing 92

9.7.1. Numerical description of the angiogram (Angioscore) 92

9.7.2. Pressure data analysis 95

9.7.3. Analysis of post-exercise pressure indices 95

9.7.4. Transit time measurement 96

9.7.5. Doppler sonagram waveform analysis 97

9.7.5.1. Qualitative analysis of the Doppler sonagram 97

9.7.5.2. Quantitative or numerate analysis of the Doppler sonagram

9.7.5.2.a Processing of the sonagram 98

9.8. Statistical analysis methods

9.7.5.2.b Selection and calculation of numerate

$\begin{array}{ll}\text { 10. RESULTS } & 103\end{array}$

10.1. Survey of the results and discussion 103

10.1.1. Angiograms 103

10.1.2. Clinical examination $\quad 105$

10.1.3. Systolic blood pressures in the limb at rest 106

10.1.4. Walking test 109

10.1.5. Ankle pressure response to exercise $\quad 110$

10.1.6. Aorto-femoral transit time measurement 111

10.1.7. Doppler signals 113

10.1.7.1. Characteristics and quality of the Doppler sonagrams 113

10.1.7.2. Qualitative Doppler scores 117

10.1.7.3. Quantitative or numerate Doppler signal parameters

$\begin{array}{ll}\text { 10.3. Diagnostic results with the selected parameters } & 129 \\ \text { 10.3.1. General diagnosis of arterial obstruction of the legs } & 129\end{array}$ 
10.3.2. Segmental diagnosis of arterial obstructions

10.3.3. Diagnosis of aortoiliac obstructive disease

10.3.3.1. Classifying parameters, classification rules and diagnostic accuracy, specificity and sensitivity

10.3.3.2. Discussion of false-positive and falsenegative classifications

10.3.4. Non-recordable Doppler signals

10.3.5. General remarks and conclusions on the use of the selected parameters in the diagnosis of arterial obstructions

11. SUMMARIZING DISCUSSION OF RESULTS, RECOMMENDATIONS AND CONCLUSIONS

12. SUMMARY - SAMENVATTING

APPENDIX

REFER ENCES 


\section{INTRODUCTION}

\subsection{REASON TO PERFORM THE STUDY}

The immediate and longterm results of aortofemoral reconstructive operations in patients with isolated aortoiliac obstruction are uniformly reported as good and more or less the same for the different surgical techniques in use (De BAKEX et al 1958; IMPARATO et al 1970 ; MOZERSKY et al 1972; GARRETT et al 1977; DARLING et al 1979). Equally satisfactory results are possible with transluminal angioplasty ("Dotter procedure") in cases of subtotal iliac obstructions (van ANDEL and KREPEL 1979; van ANDEL 1980). Subjectively the results are only good if the elliminated obstruction was of hemodynamic importance. Reports can be found about patients not improving after reconstruction of angiographically abnormal iliac segments where the preoperative hemodynamic parameters could not confirm the X-ray findings (BRENER et al 1974; LEVESON et al 1978). The reverse is also occuring. Good improvement of symptoms and parameters of limb circulation was reported if a proximal reconstruction was performed in patients with insignificant disease of the iliac segments on angiographic grounds, but whose physiological parameters for aortoiliac obstruction were positive (BRENER et al 1974). The vascular operations most frequently performed are peripheral arterial reconstructions (COUNCIL FOR HEALTH RESEARCH TNO 1980), usually femoro-popliteal bypass operations. Although many other factors can be held responsible for the patency rates of femoro-popliteal and femoro-crural reconstructions, it is generally accepted that they are at jeopardy if aortoiliac stenotic lesions are not suspected and left untouched (SUMNER and STRANDNESS 1978; TAKS 1978). CHARLESWORTH and colleagues (1975) could show that a functional parameter of the circulation like the pulsatility index of the femoral artery (a parameter to be discussed in section 6.3.3.) was of more prognostic value for the early patency rate of saphenous vein femoro-popliteal bypasses than the angiographic evaluation of the aortoiliac segment. Consequently they explored the iliac artery prior to femoro-popliteal grafting when the functional parameter indicated aortoiliac obstruction even if the iliac angiogram seemed normal.: They invariably found significant iliac obstruction in those cases. Others regularly performed succesful femoropopliteal grafting in legs of which the aortoiliac segments were considered to be moderately to severely obstructed on the angiogram, if hemodynamic tests (direct arterial blood pressure measurements) suggested non-significant proximal lesions (BRENER et al 1974; LEVESON et al 1978). In patients with multilevel obstructive disease the elimination of a significant iliac stenosis alone can relieve the symptoms by improving the circulation to an acceptable level. This is also the case if occlusions of the superficial femoral artery remain as was shown in series of so called "profunda revascularizations" (WESOLOWSKI et al 1966; GARRETT et al 1977; WARD and MORRIS - JONES 1978). On the other hand aortofemoral reconstruction is less likely te be beneficial when the aortoiliac stenosis is of minor significance compared with the coexisting distal obstruction and consequently such cases should be recognized preoperatively (BONE et al 1976; GARRETT et al 1977). In the light 
of this knowledge, obstruction in the aortoiliac pathway appears to be a very important lesion to be indentified from a vascular surgical point of view.

Until one decade ago obstructive disease in the arteries of the lower extremities was diagnosed nearly exlusively by physical examination and angiography. In recent years the evaluation of patients with vascular diseases has been improved by the introduction of a variety of noninvasive functional lesting procedures wich usually are performed in the setting of so-called "vascular laboratories" (STRANDNESS 1974; BERNSTEIN 1978; BUTH 1978). These laboratories are rapidly increasing in number and some discussion has already started about a too liberal and indiscriminate use of vascular laboratories. Their legitimate position in larger vascular clinics, however, is well established (RAINES et al 1976; STRANDNESS 1979). One of the developments that can be held responsible for the major break-through of physiologic testing in vascular patients was the widespread marketing of handy ultrasonic Doppler devices for the transcutaneous detection of blood flow velocities. Certainly, nowadays Doppler equipment occupies the first place among the instruments used in modern noninvasive arterial evaluation procedures due to its simplicity and versatility (DEAN and YAO 1976).

In view of the importance of a correct diagnosis of aortoiliac obstruction and of ultrasonic Doppler examinations in noninvasive arterial testing procedures, the present study was undertaken.

\subsection{AIM OF THE STUDY}

The aim of the present study was to determine the diagnostic value of various noninvasive ultrasonic Doppler examinations of the lower extremities in the assessment of the degree of chronic aortoiliac obstructive disease. Angiography was selected as the standard to which the results of these tests were compared. In spite of all objections to angiography as a poor method to determine the anatomical degree of arterial obstruction, particularly in the pelvic region, no better method is available in the daily clinical practice until now. In most recent studies on noninvasive vascular examination methods comparable to the present study, angiography had been used as the "gold standard" (e.g. HYLKEMA 1975; BRUIJNINCKX 1976; BUTH 1978; BERNINK 1978; BRUINS SLOT 1981). The tests performed in this study were partly established examinations, like systolic pressure measurements in the limb, using Doppler equipment for flow detection and partly some newer examinations, the value of which in the assessment of arterial disease is still controversial. The latter ones were analysis of the waveform of Doppler signal recordings (Doppler signal analysis) and the determiniation of time delays between Doppler signals (transit time measurements).

The study was designed in such a way that answers to the following questions night be expected.

- Which characteristics of the signal (signal parameters) are the most valuable ones and from which sites should the signals be recorded to diagnose the degree of aortoiliac obstruction?

- Is it worthwhile to perform Doppler signal analysis under conditions of hyperemia?

- Is the transit time measurement from the aorta to the common femoral artery of vallue for the diagnosis of aortoiliac obstructions? 
- How does the diagnostic value of Doppler waveform analysis and/or transit time measurements compare to that of normal physical examination and to systolic pressure measurements in the leg?

- Which practical combination of noninvasive ultrasonic Doppler tests allows the best agreement with angiography in the diagnosis of aortoiliac obstruction?

\subsection{CONTENTS AND DIVISION OF THE THESIS}

The first part of this thesis contains reviews from literature dealing with general aspects of chronic aortoiliac disease (Chapter 2), hemodynamics of the arterial circulation of the limbs in relation to the tests performed in the present study with special emphasis on pressures and flow velocities (Chapter 3) and principles of ultrasonic Doppler equipment and techniques (Chapter 4). This part further includes chapters about the various test modes used in this study, namely systolic pressure measurements in the limb (Chapter 5), Doppler signal analysis (Chapter 6), as well as transit time measurements and determination of parameters of Doppler waveform damping, used both alone and in combination with each other (Chapter 7). The first part of the thesis ends with a review of the value of the various Doppler examinations in the diagnosis of aortoiliac obstruction (Chapter 8). In the second part of this thesis the own study is described. Instrumentation, test methods and analytical techniques of the study are described and discussed in Chapter 9. In Chapter 10 all results are presented in detail and compared to the findings of other investigators. Chapter 11 contains a general discussion on the final results of the study and conclusions.

The clinical research for the present study was performed in the surgical department of the St. Antonius Hospital in Utrecht. Technical assistance and guidance in the spectrum analysis of Doppler signals was received from the department of Biophysics of the University of Limburg in Maastricht. (A.P.G. HOEKS, M. Sc., C.J. RUISSEN). Computerized signal analysis and statistical analyses were performed in the Institute of Medical Physics (MFI-TNO) in Utrecht and the department of Medical Informatics of the Free University in Amsterdam (both by J.L. TALMON, M.Sc). 


\section{CHRONIC AORTOILIAC OBLITERATIVE DISEASE}

In this chapter general aspects of the aortoiliac obstructive syndrome as well as the value of some methods to diagnose aortoiliac obstruction will be discussed.

\subsection{GENERAL ASPECTS OF CHRONIC AORTOILIAC OBLITERATIVE DISEASE}

\subsubsection{Terminology and definitions}

Chronic aortoiliac obstructions are, with a few exceptions, part of the clinical disease atherosclerosis obliterans (ASO), known also by such established names as peripheral arteriosclerosis or arteriosclerosis obliterans. Atherosclerosis obliterans is used here as a general diagnostic term for a chronic arterial disease of the aorta, its major branches and the large and medium-sized arteries of the extremities, particularly of the legs. As a result of the disease the lumina of these vessels may be partially or totally obliterated. A variety of pathologic findings and clinical syndromes are included in this diagnostic term (JUERGENS and BERNATZ 1980; VOLLMAR 1975). In a strict sense arteriosclerosis and atherosclerosis are definitions used in pathology. Arteriosclerosis denotes the "normal" changes in the arteries due to aging and the chronic strain of arterial blood pressure, characterized by an increase of collagen fibers, loss of elasticity and deposit of calcium in the wall. Atherosclerosis is the combination of a deposit of lipoids and other blood products in the intimal layers (in the form of atheromatous plaques) and local fibromuscular proliferations (HAIMOVICI 1977; FUSTER et al 1980).

In the term "atherosclerosis obliterans" the word atherosclerosis does not have this strict histo-pathological meaning.

\subsubsection{Pathology and etiology}

In over 95 percent of cases of chronic aortoiliac obstruction the underlying diagnosis is ASO and the primary pathologic lesions are nearly always atherosclerotic plaques, with of without mural thrombi, which cause localized stenosis or acclusion. Only rarely other degenerative arterial diseases are invalved (VOLLMAR 1975). A discussion about the probably multifactorial etiology of atherosclerosis (HAIMOVICI 1977) is beyond the scope of this study. Other, wery rarely occurring, causes of chronic aortoiliac obstruction are primary thrombosis, post-embolic occlusions, posttraumatic thrombosis, congenital aplasia, fibromuscular hyperplasia and external compression (VOLLMAR 1975).

\subsubsection{Anatomic patterns of the disease}

ASO is not evenly distributed along the vascular tree of the legs. Typical primary sites of occurrence are the distal abdominal aorta and its bifurcation, the iliac and common femoral arteries and their bifurcations, the superficial femoral 
artery at the level of Hunter"s canal, the distal popliteal artery and in diabetic patients the lower leg arteries. This unequal distribution of localization of the disease has given rise to different morphological classifications of ASO of the legs and within it to a variety of sub-classifications for the aortoiliac segment.

Usually the aortoliac segment is considered to include the infrarenal abdominal aorta, the common and external iliac arteries and the common femoral artery, down to its bifurcation in superficial and deep femoral artery.

One frequently used classification of ASO of the lower extremities distinguishes between:

- disease mainly confined to the aortoiliac region,

- disease limited to the arterial segments distal to it,

- and the cases where disease is diffusely found at both levels ("combined" of "multilevel disease").

(DeBAKEY et al 1958; IMPARATO et al 1970; HYLKEMA 1975; BRUIJNINCKX 1976; BUTH 1978; BERNINK 1978\%.

For the aortoiliac ASO a variety of sub-classifications are in use. Quite long ago DeBAKEY et al (1958) made a subdivision in complete and incomplete occlusions, which is no longer considered of much practical value by others (VOLLMAR 1975). More important is the often used differentiation between aortoiliac obstructions with and without distal arterial involvement (IMPARATO et all 1970; DARLING et al 1979). These two types seem to occur in two distinct patients' populations with a different frequency of concomitant other cardiovascular diseases and diabetes as well as differences in age and sex distribution, disease progression and life expectancy (WESOLOWSKI tet al 1966; MOZERSKY et al 1972; DARLING et al 1979).

About 75-80 percent of all patients with aortoiliac obstructive disease belong to the group with peripheral involvement (VOLLMAR 1975; DARLINGet al 1979).

Within the aortoilliac segment one can distinguish between a morphologic type with lesions confined to the proximal part of it down to the iliac bifurcation, and the type where lesions extend to the groin. The first group is probably only a less far progressed stage of the same disease process as indicated by a younger mean age of this group (IMPARATO et al 1970; DARLING et al 1979). Some authors separately classify the total aortic occlusion which is of ten considered to evolve out of bilateral occlusion of the common iliac arteries (DeBAKEY et al 1958; IMPARATO et al 1970; VOLLMAR 1975; DARLING et al 1979). This sub-classification is justified because this disease, which gives the classical LER ICHE syndrome, carries the risk of ascending thrombosis up to the level of the renal arteries which makes the indication to vascular surgery a very stringent one. Vollmar finally distinguishes between short segmental occlusions of the distal aorta or iliac arteries and the "bifurcation" type where the origin of the common iliac arteries is severely obstructed or occluded. The practical point is that the segmental type is usually better tolerated by the patient than the bifurcation type due to better collateral possibilities (VOLLMAR 1975).

Aortoiliac obstructions very often ( 70 percent) occur bilaterally (VOLLMAR 1975). No publications could be found mentioning anything about a preference of the disease for one of the two sides of the body.

Obstructive aortoiliac disease may be associated with aneurysmal disease of the abdominal aorta or iliac arteries. In 27 percent of their patients with occlusive processes at the aortic bifurcation DeBAKEY et al (1958) also found aneurysms proximal to it, whereas DARLING et al (1979) diagnosed associated narrowing of the iliac vessels in $\$ 5$ percent of their patients undergoing elective 


\subsubsection{Occurrence and frequency distribution of aortoiliac obstruction}

No data are available about the absolute incidence $(=$ number of new disease cases observed during a certain period) and prevalence (-total number of disease cases at a given time) of ASO among the total population, much less about that of aortoiliac obstruction. It may be illustrative that in a recently published report about the importance of diseases of the circulatory system in the Netherlands, which is quite detailed about some diseases, one will look in vain for specified figures about atherosclerosis of the legs (COUNCIL FOR HEALTH RESEARCH TNO 1980). Only indirect indications of the frequency are found. In the sixties van BUCHEM (1967) did a follow-up study on 918 randomly selected men, aged $40-59$ years, from one Dutch town. During a six years period 25 of them developed symptoms of intermittent claudication, whereas 4 men already had claudication at the onset of the study. This corresponds to a yearly incidence of about 45 per 10.000 in this group of men. About the same incidence figures for intermittent claudication in men were found in the well known Framingham study; women lagged behind men by 10 years to reach the same incidence (KANNEL et al 1970).

ASO occurs predominantly among men between $50-70$ years (JUERGENS and BERNATZ 1980). The overall percentage of females among patients with ASO coming to vascular surgery in greater patient's series was between 5 and 10 percent (DeBAKEY et al 1958; VOLLMAR 1975). In these series the women were particularly underrepresented among the patients with multilevel ASO. For disease only confined to the aortoiliac region a quite equal distribution among the sexes was found. Many of the female patients with aortoiliac ASO appear to have had an artificially induced menopause (DARLING et al 1979). Van VROONHOVEN and MULLER (1978) considered ASO in pre-menopausal women less rare than is often believed; they described a characteristic group of young female patients, all having used oral contraceptives during longer periods of time with localized stenosis in the distal abdominal aorta.

Among patients with diabetes mellitus both the prevalence and the severity of ASO are higher than in the rest of the population, whereas diabetics develop symptoms of ASO on an average 10 years earlier than non-diabetic ASO patients (JUERGENS and BERNATZ 1980).

In about one-third of cases with ASO of the lower extremities, seen by physicians, the aortoiliac segments are affected. Threefourth of these patients with aortoiliac ASO are between 40-59 years of age (VOLLMAR 1975).

\subsection{THE DIAGNOSIS OF CHRONIC AORTOILIAC OBSTRUCTION}

If a patient is suspected of arterial obstruction the following questions are raised:

- Is there really arterial obstruction?

- How are the obstructive lesions localized?

- How serious are the functional impairments caused by the obstruction(s)? A complete physical examination will usually answer the first two questions in patients with severe aortoiliac obstruction. With less severe disease and particularly in patients with multilevel disease the mere physical examination will leave the second question unanswered. In those cases complementary tests 
are needed to complete the picture of the disease. Neither routine physical examination nor angiography will give a good impression of the functional disturbances caused by the disease (MOORE and HALL 1971; BRENER et al 1974). Hence supplementary tests are also required to cover this aspect.

Angiography is rarely necessary to establish the diagnosis and should not be considered as a routine diagnostic procedure (JUERGENS and BERNATZ 1980). Its aim is to provide an anatomic "map", after the decision to perform a vascular operation in principle has already been taken (HYLKEMA 1975; BRUIJNINCKX 1976). This map will confirm the presence of lesions suitable for surgery or interventional radiology (Dotter's angioplasty), allow a direct approach to the involved segment and help in the selection of the therapeutic method to be used.

\subsubsection{Symptoms and signs}

Sometimes aortoiliac obstruction can be diagnosed in patients without symptoms if they have isolated segmental occlusions of the common or external iliac artery with a good collateral circulation (VOLLMAR 1975). In all others a predominant symptom of the disease is pain sensation of intermittent (intermittent claudication) or persistent (rest pain) character.

Intermittent claudication is usually the earliest and one of the most specific symptoms of the disease and manifest in nearly all symptomatic patients (WESOLOWSKI et al 1966; VOLLMAR 1975; DARLING et al 1979; JUERGENS and BERNATZ 1980). In general, there is no direct correlation between the level of occlusive disease and the localization of the pain of intermittent claudication. A history of only proximal, femoro-gluteal, pain is nearly always an indication of aortoiliac obstruction in the absence of significant occlusive disease below the inguinal ligament. On the other hand, quite some patients with isolated aortoiliac disease will only complain of calf claudication (WESOLOWSKI et al 1966; VOLLMAR 1975; DARLING et al 1979; FAIRBAIRN 1980).

Rest pain is a symptom of severe obstructive disease, only occurring in patients with aortoiliac and peripheral obstructions in series (WESOLOWSKI et al 1966; DARLING et al 1979; FAIRBAIRN 1980). It may occur rarely in patients with isolated aortoiliac occlusions of acute origin (VOLLMAR 1975). The pain is usually experienced in the digits, but also in the foot and the lower leg, often at night and thus interfering with sleep.

A next grade of severity of obstructions is indicated by the presence of gangrene and/or ulcerations, often associated with severe pain except in patients with advanced neuropathy (diabetes). Further details about ischemic rest pain and the characteristics of ischemic ulceration can be found in standard textbooks (FAIRBAIRN 1980; JUERGENS and BERNATZ 1980).

A symptom considered to have a specific relation to aortoiliac occlusive disease is male impotence. This is found to occur in about 20-29 percent of patients with aortoiliac obstructions (VOLLMAR 1975; van VROONHOVEN 1977), and is suggestive of an impediment to inflow into the hypogastric arteries due to proximal aortoiliac obstructions. It should, however, be recognized that impotence is also related to the age of the patient (DARLING et al 1979), whereas the lack of return of potency after succesful revascularizations of the internal iliac arteries suggests that also other factors are reponsible for it (VOLLMAR 1975). In women, suffering from aortoiliac obstruction, no symptoms corresponding to the male impotence have as yet been recognized. 
Apart from various trophic changes - which will not be discussed bere and for which the reader is referred to the textbooks (FAIRBAIRN 1980; JUERGENS and BERNATZ 1980) - the most important signs to the clinician in the diagnosis of obstructive arterial disease are the character of arterial pulsations on palpation and the presence and the character of bruits as heard with the stethoscope. It can be stated in general that arterial pulsations of the femoral, popliteal and posterior tibial artery are never absent in a resting patient in a warm environment, except as a result of occlusive arterial disease (JUERGENS and BERNATZ 1980). Absence of pulsations of one or both dorsallis pedis arteries is possible on a congenital base and cannot be considered, therefore, as a sign of obstruction (BARNHORST and BARNER 1968; JUERGENS and BERNATZ 1980). A weak or absent femoral pulse under resting conditions certainly denotes severe proximal obstruction. The reverse, however, does not hold: with well palpable femoral pulsations a considerable degree of proximal obstructive disease may be present in cases associated with severe occlusive disease of the superficial and deep femoral artery (BLAISDELL and GAUDER 1961; WESOLOWSKI et al 1966; JUERGENS and BERNATZ 1980). This phenomenon can be explained by an augmentation of the otherwise damped pulse pressure by the peripheral occlusions. Therefore, a palpable femoral pulse in association with severe occlusive disease of the femoral bifurcation does not necessarily signify lack of proximal disease. A femoral pulse which disappears after a period of exercise or cuff occlusion at the thigh is an indication of stenosis of a major proximal artery (KEITZER et al 1965; JUERGENS and BERNATZ 1980). During the period of arrest of distal flow by the cuff in the test just mentioned often a marked increase in the strength of the femoral pulse may be noticed (KEITZER et al 1965). Because considerable aortoiliac obstructions are not always suspected on the basis of femoral pulse palpation this test is not a sensitive indicator of disease (BRUIJNINCKX 1976). Another disadvantage is the fact that grading of femoral pulses is very subjective and unreliable, particularly in obese patients. Even experienced observers will rarely agree about the quality of a pulse (BRENER et al 1974).

A bruit is the result of turbulences in the bloodstream and usually occurs urder resting conditions only if a diameter stenosis of at least 50 percent is present in the artery. Therefore, a bruit is a good indication of significant stenosis and at the same time of the existence of a residual lumen in the artery. In healthy persons bruits are never heard over the arteries of the leg. A bruit also indicates with great certainty the localization of the stenosis as the bruit originates at the site of constriction and is only propagated downstream and never upstream (FRANSEN et al 1969). The intensity of the murmur is not strictly proportionate to the magnitude of the constriction, although a very intensive bruit probably indicates a tight stenosis (HAIMOVICI and ESCHER 1955). With less severe stenoses in the iliac arteries, bruits may be absent at rest but be provoked by some exercise. Such cases very often have aortoiliac abnormalities which show-up on angiograms (WESOLOWSKI et al 1966). The search for aortic, illiac and femoral bruits at rest and after exercise is an indispensable part of the physical examination of patients suspected of aortoiliac obstructions.

\subsubsection{Angiography}

At present no serious discussions exist about the necessity of an angiographical 
imaging of the pelvic and limb arteries, once the decision to perform operative treatment has been taken in principle (BONE et al 1976).

Usually a translumbar aortography (TLA) or a catheterization method (transfemoral retrograde or transaxillar orthograde) will be performed. After a TLA additional direct needle contrast injections into the femoral artery (femoral angiography) may be needed for a good definition of the lower leg arteries. In many cases of aortoiliac obstructions the use of a transfemoral catheter technique (Seldinger) is less advisable because of the chance of injuring the pathologic arterial wall, dislodging thrombi or plaques or aggravating thrombus formation. This often makes TLA the primary choice in aortoiliac angiography (MADDISON 1977; ROBICSEK 1978; FULTON et al 1980).

Radiological signs of stenosis are a reduction in the width of the lumen image, increased local transparency of the contrast film and the appearance of collaterals where an obliteration is apparently absent (MANSJOER et al 1976). In the single plane projections of aorto-angiography for the aortoiliac system, high grade luminal reductions in the dorso-ventral direction may not be visible on the pictures if the transverse vessel diameter is not reduced or if a high dose of contrast is used (MOORE and HALL 1971; KÖHLER 1973; MANSJOER et al 1976; WATERS et al 1977; CRUMMY et al 1978). Other reasons for the nonvisualisation of stenoses are superimposition of other arteries and the fact that the iliac arteries are seen foreshortened (CASTANEDA-ZUNIGA et al 1976). Unfortunately the majority of plaques are localized on the dorsal and ventral side of the aorta and iliac arteries, as could be shown in threedirectional angiographic (KÖHLER 1973) and pathological studies (MOORE and HALL 1971).

It can, therefore, be almost considered and axion that disease in the aortoiliac segments is more severe at surgical exploration than indicated by the (routine) angiography (WESOLOWSKI et al 1966; MOORE and HALL 1971; BRENER et al 1974; FARIS and JAMIESON 1975). To partially overcome this disadvantage, multiplane projections can be used. For the iliac vessels, these should be oblique to prevent superimposition of the illiac arteries. A major disadvantage of this approach is the need for a catheter technique and multiple contrast injections if no special X-ray equipment is available (KÖHLER 1973; SETHI et al 1975; CASTANEDA-ZUNIGA et al 1976; CRUMMY et al 1978).

A drawback of angiography is the absence of functional information offered by it. Interpretation of angiographic data in a physiologic sense, particularly for the iliac segments, can lead to surgical errors (MOORE and HALL 1971; BRENER et al 1974; CASTANEDA-ZUNIGA et al 1976). In case of multillevel disease of the aortoiliac and the femoral arteries it is difficult to decide on the evidence of the angiogram alone which segment should be operated upon (HARRIS et al 1974).

\subsubsection{Indirect blood pressure measurements}

These measurements constitute an important part of the examinations used for this study and are discussed in detail in Chapter 5 and in the sections 8.1,8.4 and 8.5 .

\subsubsection{Direct arterial blood pressure measurements}

A valuable test of the functional importance of angiographically equivocal 
aortoiliac abnormalities is the measurement of the blood pressure drop which may occur across it. The theoretical backgrounds of the pressure fall across stenotic lesions will be given in section 3.5.2. In such a test intraarterial pressures are measured both proximal to the aortoiliac segment, for example, in the abdominal aorta or in the brachial artery and at the distal end of the segment. for instance, in the common femoral artery. Frequently measurements are performed during the angiography procedure through the catheters or needles already positioned for the contrast injections (CASTANEDA-ZUNIGA et al 1976; BARDACH 1978;UDOFF et al 1979). Two aspects are considered of importance in these measurements: the simultaneous measurement of both pressures and the employment of a hyperemic reaction, induced, for instance, by the intraarterial administration of a vasodilator drug.

In the case of significant aortoiliac obstruction a systolic pressure gradient of at least $20 \mathrm{mmHg}$ is found during hyperemia (CASTANEDA-ZUNIGA et al 1976; UDOFF et al 1979 ).

Alternative methods are determination of the common femoral artery pressures alone and comparison of these pressure before and after calf exercise (MOORE and HALL 1971) or before and after a period of circulatory arrest by thigh cuff occlusion (BRENER et al 1974).

These direct pressure measurement tests are always positive in case of demonstrable angiographic aortoiliac obstructions. In cases where the angiography seems normal but the pressure measurements are indicative of obstruction the pressure data should be preferred to rely on (WESOLOWSKI et a] 1966; BRENER et al 1974). The tests are also of value in the presence of distal obstructive lesions (BRENER et al 1974).

\subsubsection{Pulse waveform analysis}

The pulsatile inflow of blood into the leg causes synchronous changes in segmental volume and electric impedance of the limb as well as cyclic flow velocity changes in the arteries.

The volume changes can be recorded plethysmographically as a volume pulse wave (BUTH 1978). A general survey of available plethysmographic techniques and their fields of application are to be found in the theses of HYLKEMA (1975), BUTH (1978) and BERNINK (1978).

The electric impedance changes of a limb segment are recorded with the aid of an impedance plethysmograph (or rheograph) (SCOTT 1977); this instrument has not received wide acceptance in the vascular laboratories as yet (Van De WATER et al 1971).

Flow velocity changes in the greater arteries of the leg can be recorded as flow velocity curves with Doppler ultrasound equipment (see Chapter 4).

The volume pulse waves, the impedance curves and the velocity pulse waves all have contours or patterns which are comparable to the pressure curves obtained by direct arterial cannulation of the greater arteries or are strongly related to them (RAINES 1977 and section 3.3.2.); they show typical deviations from the normal in cases of obstructive arterial disease. The different methods for analysis of the Doppler velocity waveforms will be discussed extensively in Chapter 6; they are not fundamentally different from the methods used for the analysis of the other pulse waves. The place of Doppler signal analysis in the diagnosis of aortoiliac disease will be discussed in Chapter 6 and in the sections 8.2, 8.4. and 8.5 . 
An important plethysmograph from a clinical point of view is the mercury-insilastic (rubber) strain gauge plethysmograph (STRANDNESS 1969; BARENDSEN 1973; SUMNER 1977). However, this is more frequently used in calf flow measurements (section 2.2.6.) than in pulse wave analysis. An exception is the digital plethysmography (STRANDNESS 1969; SUMNER 1977) in which the waveform is an important parameter. Other groups use photo-electric scatter eyes for this digital plethysmography with similar results (HYLKEMA 1975; BERNINK 1978). For the diagnosis of very peripheral arterial obstructions and for vasos pastic disorders, digital plethysmography is a good technique (SUMNER 1977) but for the specific functional evaluation of aortoiliac obstructions it is hardly of any use as was shown by HYLKEMA (1975).

Another plethysmographic device which came into widespread use is the Pulse Volume Recorder (PVR) developed by Darling and Raines, which is an air filled rubber cuff plethysmograph (DARLING et al 1972; RAINES et al 1976; RAINES 1977; BUTH 1978). The primary waveform parameter in PVR reading is the well reproducible amplitude of the pulse wave (RAINES 1977; BUTH 1978). Using this PVR amplitude, obtained at various levels of the leg, in combination with segmental systolic pressure measurements both at rest and post-exercise (necessitating the use of multivariance analysis with a classification rule with 9 different parameters!) an overall correct anatomic diagnosis of aortoiliac obstructive disease could be made in 70-80 percent of the cases; in the case of iliac stenoses the score was considerably lower (BUTH 1978).

Since in many hospitals mechanic oscillography (HYLKEMA 1975; BERNINK 1978 ) is still part of the work-up of patients with peripheral arterial disease, some words should be spent on this method. It is a non-standardized method, the recordings of which are not suitable for waveform analysis. It allows only some conclusions about left-right differences and gives a rough indication about the level of the obstructions, information also obtainable by a good clinical examination (JUERGENS and BERNATZ 1980; RAINES 1977). It is impossible to determine on the basis of the upper leg oscillogram, whether there is a hemodynamically significant aortoiliac obstruction (HYLKEMA 1975).

\subsubsection{Blood flow measurements}

In clinical practice (semi-)noninvasive blood flow measurements to evaluate the leg"s circulation are nearly exclusively performed at the level of the calf. At present two techniques are most frquently employed: venous occlusion plethysmography (BARENDSEN 1973; HILLESTAD 1977) and isotope clearance methods (1.ARSEN 1972; LASSEN and HOLSTEIN 1974). Both these techniques give their results in $\mathrm{ml}$ bloodflow/100 gram tissue/minute. It is a fully accepted opinion, found in all publications, that flow measurements at rest have no value in the diagnosis of arterial obliterative disease, as they are equal in normal and in ischemic limbs (see e.g. HILLESTAD 1963a, 1977; BARENDSEN 1973; STRANDNESS and SUMNER 1975, p. 233-234; HYLKEMA 1975). Differentiation between normals and patients only occurs during hyperemia caused by muscle exercise or temporary circulatory arrest in the limb.

Important plethysmographic hyperemic flow parameters, indicative of arterial obstruction, are a low peak blood flow, a delay in the timing of this peak flow, and a prolonged hyperemic response (HILLESTAD 1963a; STRANDNESS and SUMNER 1975, p. 234; HYLKEMA 1975; BERNINK 1978). A considerable overlap of values for various morphologic groups of obstructive arterial disease, 
however, makes these parameters useless for the anatomic diagnosis of the obstructions (HYLKEMA 1975; BERNINK 1978). The absolute now values during and after exercise or after release of an occlusion as calculated by the isotope clearance method are presenting such an overlap and poor reproducibility that they are of no value for individual patient evaluation (TONNESEN 1968; LASSEN and HOLSTEIN 1974; KITSLAAR and van VEENENDAAL 1977). For this reason the shape of the isotope washout curve became the more relevant parameter to evaluate the functional status of the arterial system (LEWIS et al 1972a, 1972b; LASSEN and HOLSTEIN 1974; SMITH et al 1977).

Quite in general, the types of hyperemic flow response curves are correlated to the angiographic and clinical severity of obstructive arterial disease, but due to a substantial overlap they do not allow an anatomic diagnosis of the disease (SUMNER and STRANDNESS 1969; LEWIS et al 1972a, 1972b; SMITH et al 1977). Interesting is the close inverse relationship between the postexercise systolic blood pressures at the ankle and the calf flow values found by SUMNER and STRANDNESS (1969) and confirmed in their studies by LEWIS and coworkers (1972a, 1972b). They considered these pressure measurements just as valuable or even better for patients evaluation as the more cumbersome and expensive flow measurements.

It may be concluded that flow studies during hyperemia allow a very general estimation of the functional importance of obstructive arterial disease but lack specific information about anatomic or functional involvement of the aortoiliac segment.

\subsubsection{Measurement of aorto-femoral transit times}

The short time for a pressure or velocity pulse to travel along the aortoiliac segment - which normally is in the order of 20 milliseconds - may be changed by obstructive disease (see Chapter 7).

Practically the only way to measure noninvasively the aorto-femoral pulse propagation time is by the use of Doppler equipment. In peripheral limb segments also other pulse-sensing devices could be used for this purpose. In the sections 7.2,7.4,7.5, 8.3,8.4 and 8.5 diagnostic and other aspects of pulse propagation or pulse transit times determined by the Doppler method will be discussed.

Different is the measurement of the time needed for a fluid solution, injected into the bloodstream, to be transported from the abdominal aorta to the groin, which normally takes about $0.5-1$ second. HURLOW et al (1978) described a "dynamic isotope angiography", in which an intravenously injected isotope bolus was followed during its passage through the aortoiliac segment. Significant differences were found in these transit times for various grades of severity of aortoiliac obstruction as checked by routine angiography. This method consequently might be of value in the diagnosis of aortolliac obstruction. 



\section{HEMODYNAMIC AND PATHOPHYSIOLOGICASPECTS OF THE ARTERIAL CIRCULATION OF THE LIMBS}

\subsection{BASIC PRINCIPLES}

Blood is moving in the arterial system in response to local differences in total fluid energy. This energy content is made up of the kinetic energy ( $K$ ) imparted to the blood by the contraction of the heart and of potential energy which is composed of the intra-vascular pressure (P) and the gravitational potential energy (G) (KEITZER et al 1965; SUMNER 1977). Kinetic energy (K) is a function of the specific density of the blood ( $\rho)$ and its velocity (v) formulated as,

$$
K=1 / 2 \cdot e \cdot v^{2}
$$

Putting all components together, the total fluid energy per volume of blood (E) can be expressed as,

$$
\mathrm{E}=\mathrm{P}+\mathrm{G}+\mathrm{K}
$$

Potential energy $(P+G)$ can be converted to kinetic energy $(\mathrm{K})$ and vice versa. For frictionless, steady, fluid flows through straight pipes this relation between $P, G$ and $K$ is governed by the classical BERNOULLI's Principle of continuity of total energy content along the conduit (see e.g. KEITZER et al 1965; McDONALD 1974 p. 35; de BOER 1978). In physiologic systems the BERNOULLI's theorem is not valid because the flow is not steady but pulsatile, the fluid is not frictionless but approximates the characteristics of a Newtonian fluid (STRANDNESS and SUMNER 1975, p. 81-84; de BOER 1978, p. 66) and the bloodvessels are not straight pipes. Yet, the principle provides some approximation of the relationship between pressure and flow velocity if the energy dissipation of the circulation is taken into account (KEITZER et al 1965; SUMNER 1977). When comparing the energy content of a volume of blood at a proximal piont (Prox) in the circulation with that at a more downstream site (Dist) the following equation is valid (SUMNER 1977):

$$
\mathbb{E}_{\text {(Prox })}=\mathbb{E}_{(\text {Dist })}+\text { "Lost"energy }
$$

In the vascular system lost energy of moving blood is dissipated as heat (STRANDNESS and SUMNER 1975, p. 78; SUMNER 1977). In supine subjects the gravitational term (G) in equation (2) may be cancelled. This allows equation (3) to be written as:

$$
P_{\text {(Prox })}+1 / 2 e \cdot v_{(\text {Prox })}{ }^{2}=P_{(\text {Disl })}+1 / 2 e \cdot v_{(\text {Dist })}{ }^{2}+\text { heat }
$$

It means that increase of velocity of flow at a given place due to any factor is accompanied by a drop of the arterial pressure at that very place and vice versa.

The energy losses associated with the blood flow in the normal, healthy, arterial system are due to viscosity of the blood, and disturbance of flow profilles (SUMNER 1977).

The main cause for depletion of total fluid energy is turbulence of flow, which can be defined as an irregular flow condition in which the velocity vectors of small blood volumes show a random distribution both in time and in space. Turbulence is unlikely to occur in normal larger arteries except as short-lived 
flasthes during peak systole, although in large portions of the circulatory system the blood flow is on the verge of turbulence. Energy losses resulting from flow disturbances or turbulence are a function of the square of the flow velocity (STRANDNESS and SUMNER 1975).

The total fluid energy in the greater conduit arteries - measuring over $0.4 \mathrm{~cm}$ diameter (STRANDNESS and SUMNER 1975, p. 74) - is mainly made up of the potential energy of intraarterial pressure, whereas the kinetic energy is only a negligible fraction of it (SUMNER 1977; see also Figure 3.3). The total loss of fuid energy in the normal arterial system is quite small as illustrated by the mean pressure drop along the entire arterial system from the heart to the arterioles which is only about $10 \mathrm{mmHg}$ (KEELE and NEIL 1963, STRANDNESS and SUMNER 1975, p. 74, SUMNER 1977).

In the next sections the two most important components of hemodynamic energy, pressure and flow velocity, and their relationship will be discussed both for the normal arterial system and for pathological conditions like stenosis.

\subsection{NORMAL PULSATILE ARTERIAL BLOOD PRESSURES}

In the normal arteria» system the intravascular pressure increases with each cardiac contraction from around $80 \mathrm{mmHg}$ in diastole to $120 \mathrm{mmHg}$ or more in systole. At any place in the arterial system the instantaneous pressure values can be plotted against time. This results in a characteristic pressure pulse wave (see e.g. Figure 3.1). Its basic features are described in standard textbooks of physiology and in many publications about hemodynamics (e.g. KEELE and NEIL 1963; MCDONALD 1974; STRANDNESS and SUMNER 1975; de BOER 1978).

This pressure wave - like many other periodic phenomena in biological systems - may be decomposed into a number of sine or cosine waves, called harmonics, by a so called Fourier analysis (ATTINGER et al 1966; O'ROURKE and TAYLOR 1966; MCDONALD 1974; STRANDNESS and SUMNER 1975; CAVE et al 1976, FARRAR et al 1977). The harmonics' frequencies are integral multiples of the fundamental frequency or the heart rate. The amplitude of each harmonic is called modulus. Generally the various sinusoids of harmonics are not in phase with each other. Considering a complete cycle to occupy 360 degrees, the phase shift of each harmonic can be expressed as an angle of a certain number of degrees. Each pressure pulse (and the same applies to flow pulses) can thus be described in Fourier analysis by both a certain limited number of harmonics, each with their modulus and phase angle, and a mean level. Knowledge about Fourier series of the pressure pulse wave facilitates the understanding of the several changes which occur in its general form as it travels from the heart to the periphery.

The factors influencing the shape of the arterial pressure curve are the force and frequency of the cardiac contractions, the elasticity and dimensions of the major and medium-sized arteries and the status of the peripheral arterioles, determining the peripheral resistance. The predominant cause of distortion of the pressure pulse wave shape is reflection of the travelling pulse wave against bifurcations, branching points and the peripheral small vessels or the arterioles (vessels below $1 \mathrm{~mm}$ in diameter) (O'ROURKE and TAYLOR 1966, WESTERHOF et al 1972; McDONALD (chapter 12) 1974; STRANDNESS and SUMNER 1975 , p. 87; de BOER 1978). At any point in the arterial tree the amplitude of the pressure pulsation is the sum of forward travelling and of reflected waves, which 
- by interference - results in an overall increase of the pulse wave amplitude towards the periphery. A second cause for the changes is the amplification especially of the modulus of the first two harmonics of the pressure wave as a reaction to increasing vessel impedance (section 3.4) towards the periphery determined by lumen area as well as vessel wall thickness and elasticity (STRANDNESS and SUMNER 1975). On the other hand all waveform moduli are subject to damping due to the visco-elastic character of the wall and the viscosity of the blood. Especially the high frequency harmonics disappear due to damping. Finally, minor changes of the waveform are caused by differences in travelling speed between the successive harmonics. The higher harmonics travel faster than the fundamental wave, a phenomenon know as dispersion (McDONALD 1974, p. 313; STRANDNESS and SUMNER 1975, p. 87). Due to all these mechanisms the following changes of the pressure pulse wave may be noticed on its way from the thoracic aorta to the lower leg arteries: an increase of the maximum amplitude and pulse pressure, a steepening of the systolic upstroke and disappearance of its anacrotic notch, disappearance of the incisura on the downslope side and the appearance of a diastolic dip followed by a second positive deflection known as the diastolic wave in the femoral artery and beyond.

\subsection{NORMAL PULSATILE ARTERIAL VELOCITIES}

\subsubsection{The relation between pulsatile arterial pressure and flow}

The mathematical backgrounds of the following section can be found in McDONALD, chapter 6 and 13, (1974) and in STRANDNESS and SUMNER (1975).

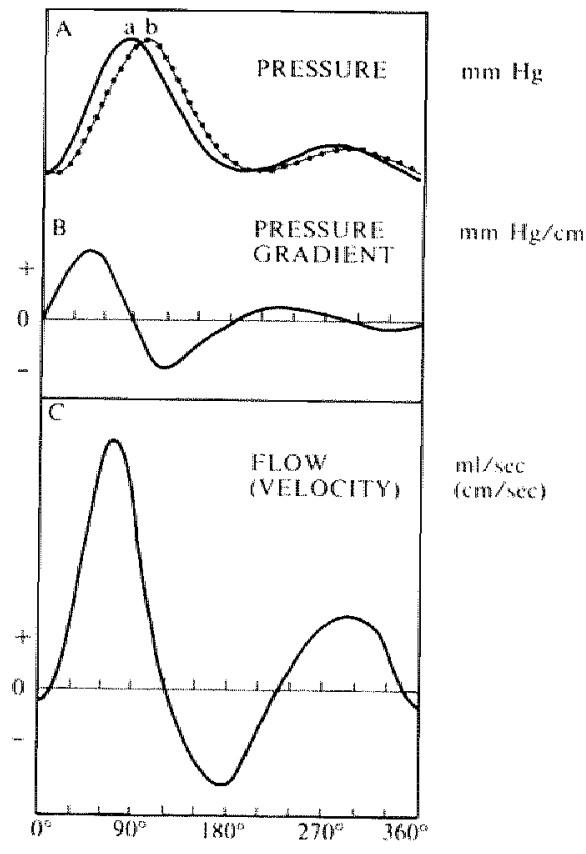

\section{FIGURE 3.1}

Phasic relation between the pressure pulse wave and the velocity pulse wave.

The figure represents one cardiac cycle $\left(360^{\circ}\right)$ in a normal femoral artery.

A. Two pressure waves recorded a short distance apart (a. upstream, b. downstream).

B. The pressure gradient derived by substracting the pressure at b from that at a divided by the distance $a-b$.

C. Flow (velocity) pulse wave resulting from the phasic positive and negative pressure gradients.

Notice the phase difference between pressure and flow.

(Adapted and redrawn from McDONALD. D. A.: "Blood Flow in Arteries", 2nd Edition. Arnold (Publishers) Lid, London, 1974. Figures 6.2, 6.11). 
In Figure 3.1 A two pressure waveforms as recorded in the femoral artery a short distance apart allong the artery, are represented. Since the mean pressure drop over this short distance is negligible the pressure waves are indentical. But because of a finite period of time between the arrival of the pressure wave at the proximal and at the distal site, the curves are out of phase, resulting in an instantaneous pressure gradient between these sites (Figure 3.IB). The direction and magnitude of blood flow (or flow velocity) are a function of this pressure gradient. Therefore, the volume flow and flow velocity versus time curves (Figure $3.1 \mathrm{C}$ ) resemble the pressure gradient curve. As may seen from Figure 3.1 rapid changes in pressure gradient from positive to negative occur during one cardiac cycle. A negative pressure gradient may result in llow reversal. Indeed, flow reversal in diastole is a common aspect of blood flow in peripheral arteries. The fact that not allways a reverse flow component is found is attributable to the inertia of the moving blood volume. In case of a normal peripheral resistance a negative pressure-gradient causes a rapid deceleration of blood flow, but only if the gradient remains reversed after the forward flow has been brought to a stand-still it leads to a reversal of flow. This usually is the case in the arteries of the leg.

\subsubsection{Flow velocity pulse wave}

A flow (velocity) versus time or flow pulse wave can be derived at any site along the arterial system on the analogy of the pressure pulse wave on which it depends (section 3.3.1). The same mechanisms which change the shape of the pressure pulse wave (section 3.2) can be considered to change the shape of the flow pulse curve on its way along the arteries. Marked differences, however, exist between the flow pulse wave and the pressure pulse wave. While the pressure pulse wave increases, the flow pulse wave progressively decreases in apmlitude towards the periphery (MCDONALD 1974, STRANDNESS and SUMNER 1975). The normal flow pulse waveform as recorded in most of the arteries of the leg shows a small steady component and large cyclic fluctuations or, in other words, its oscillates with large sweeps around a low mean, usually even swinging from positive to negative. The pressure pulse wave on the contrary oscillates around a high mean with amplitudes which are small compared to that mean. A very characteristic feature of most flow pulse waves of healthy peripheral arteries under resting conditions is the presence of a reverse flow component during part of the cycle (section 3.3 .4 and Figures 3.1 and 3.2 ).

\subsubsection{Flow velocity profiles}

The just given description of the flow velocity pulse (section 3.3.2) is a simplification of the matter in so far as it does not take into account the fact that a range of different flow velocity waveforms might be recorded along the cross section of an artery. The instantaneous flow velocity vectors of small fluid volumes in the centre of the vessel differ in magnitude and sometimes even in direction from the vectors near the wall or at intermediate position. The shape of the front of all the velocity vectors across any diameter of the vessel is called the velocity profile and presents the velocity distribution over the crosssectional area of the blood vessel. For steady flow of a viscous fluid through a long straight and rigid cylindric pipe the velocity profile has a parabolic shape, 
the liquid in the axis of the tube moving faster than that near the wall. Theoretically the "particles" of fluid can be considered to mowe in cylindrical laminae of indentical velocity, the laminae in contact with the wall being stationary and the successive layers more to the central axis slipping with an increasing velocity against the viscous friction of the laminae outside it (MCDONALD 1974, chapter 2; STRANDNESS and SUMNER 1975, chapter 1). Hence this flow type, obeying Poiseuille's Law, is called "laminar". With pulsatile flow a parabolic profile is never really attained and certainly not in the complicated, branching and tapering, conduit which the human arterial system is (MCDONALD 1974, p. 105, SUMNER 1977). There the profiles are continuously changing shape during each cardiac cycle, approaching at one moment a parabolic configuration and at other moments becoming nearly flat. Also changes of direction do occur during one cycle in many arteries and the profiles may assume skewed or otherwise asymmetric forms.

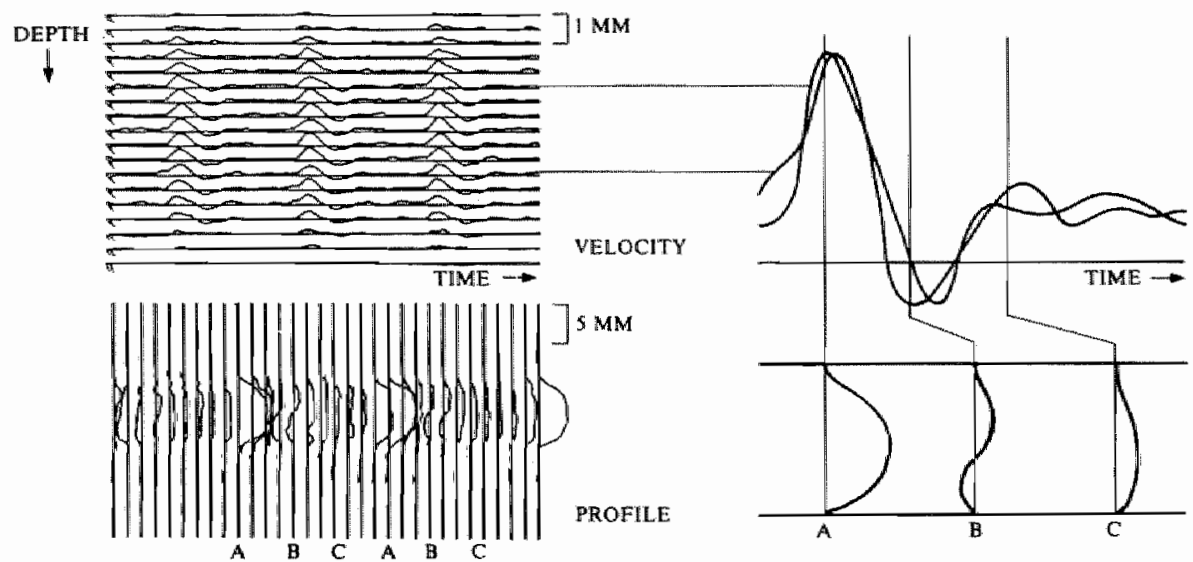

FIGURE 3.2

Relationship between flow velocity as a function of time and the flow profile.

In the upper left of the figure the "velocity mode" output and in the lower left the "profile mode" output of a multigate pulsed Doppler recording of a normal common femoral artery are shown. At a larger scale two velocity-versus-time waveforms of the velocity output are redrawn in the upper right of the figure with the corresponding profiles at times $A, B$ and $C$ in the right lower part. Notice the asymmetric, skewed, aspect of the profile in this case.

Examples of femoral artery velocity profiles are shown in Figure 3.2. They were obtained with multigate pulsed Doppler systems (HOEKS et al 1981; HOEKS 1982). In the inst rument two output modes are available: one of velocily distribution as a continuous function of depth at discrete time-intervals (profilemode) and one of velocities in a number of adjacent sample volumes as a continuous function of time (velocity pulse mode). In Figure 3.2 both outputs are shown. 


\subsubsection{Reverse flow}

For several reasons the reverse flow phase of the cardiac cycle deserves some special discussion. Since the determination of blood now velocities could develop to a routine method due to the widespread introduction of simple ultrasonic Doppler equipment, many clinicians have become aware of this phenomenon which was already familiar to physiologist for a long time. As will be shown later (section 6.2.2) the presence or absence of reverse flow is an important criterion in the classification of Doppler signals and hence of arterial pathology. Besides, the knowledge of what is happening in the vessel regions where forward and reverse flow are quickly alternating may give insight in the pathogenesis of atherosclerosis. High shear rates of blood flow have been held responsible for endothelial surface damage and may play a role in atherogenesis (quotations from literature by STRANDNESS and SUMNER (1975) and GOSL.ING (1976)).

Due to the effect of viscosity the fluid layers near the wall always have the lowest momentum. Therefore, they easily reverse when the pressure gradient reverses. Indeed, flow reversal during each cardiac cycle appears to start near the wall while the central fluid volumes still move forward (STRANDNESS et al 1969; RITTENHOUSE and STRANDNESS 1971; YAO and BERGAN 1974; MCDONALD 1974; RITTENHOUSE et al 1976). Forward and reverse components of flow transiently co-exist in one cross-section of an artery at the time of flow conversion. By simultaneous registration of forward and reverse flow at successive sites along the arteries it could be shown experimentally that the reverse flow phase was propagated from proximal to distal after the preceding forward flow phase and hence cannot be considered as a simple rebound phenomenon from the periphery (MATSUO et al 1973; NIMURA et al 1974). The reversed flow which starts near the vessel wall does not reach its highest velocity there. Maximum retrograde velocities occur in the centralfluid core(McDONALD

1974; Figure 3.2). The magnitude of reverse flow components is not evenly distributed along peripheral arteries. In larger arteries, they are generally greater in the middle section than in the proximal or distal parts. For instance a greater degree of reverse flow velocity js found in the iliac arteries than in the terminal abdominal aorta (RITTENHOUSE et al 1976). Also in the brachial artery it appears more often than in the radial artery (RITTENHOUSE and STRANDNESS 197॥; MATSUO et al 1973; NIMURA et al 1974).

Certainly the most important factor influencing the degree of flow reversal is the wave reflection against bifurcations and the arterioles. Consequently the level of peripheral vascular resistance or impedance (section 3.4) and its changes appear to be the most important determinants of the degree of flow reversal. In healthy, normal, extremity arteries where flow reversal is present during basal conditions, the reverse component can be greatly accentuated by induction of vasoconstriction, for instance by low environmental temperature, emotional status or administration of vasoconstricting agents (RITTENHOUSE et al 1976; $D E A N$ and YAO 1976). Induction of peripheral vasodilatation for instance by exercise, lumbar sympathectomy or injection of vasodilating agents causes the reverse flow phase to disappear (STRANDNESS et al 1969; YAO 1970; YAO and BERGAN 1974; RITTENHOUSE et al 1976; DEAN and YAO 1976). In patients with aortic valve incompetence regurgitation of flow may be responsible for a high reverse flow component in the femoral arteries (RITTENHOUSE and STRANDNESS 1971). 
Pressure and flow pulse waves are closely related (section 3.3.1.). A special relation between these waves is found by determination of the ratio of the modulus of each pressure wave harmonic to the modulus of the corresponding flow wave harmonic after Fourier analysis. This ratio is called the impedance and represents the opposition to pulsatile flow offered by the vascular bed distal to the place of wave recording (O'ROURKE and TAYLOR 1966; MCDONALD 1974, p. 142; STRANDNESS and SUMNER 1975, p. 88; CAVE et al 1976; FARRAR et al 1977). For each harmonic a corresponding impedance can be calculated. The ratio of mean pressure to mean flow is sometimes referred to as the impedance at zero frequency and answers the definition of resistance (STRANDNESS and SUMNER 1975; SUMNER 1977; de BOER 1978). This terminology now used in hemodynamics has been borrowed from the language of electricity. In the strict sense the term hemodynamic "resistance" should be confined to steady flow under which condition the analogy to Ohm's Law for d.c. electric current is valid. The term "impedance" ought then be reserved for pulsatile flow conditions on the analogy of a.c. electric current (MCDONALD 1974, p. 142). As mentioned above it is common use to speak about resistance to blood flow also under pulsatile physiologic flow conditions when comparing the means of arterial pressure and flow.

Studies on the magnitude of the peripheral vascular resistance of the legs show the following (LUDBROOK 1966; STRANDNESS 1969; SUMNER 1977). The total limb resistance is composed of the segmental resistance and the runoff resistance. Segmental resistance refers to the resistance of iliac, femoral and crural conduit arteries in the healthy legs whereas in obstructive disease it primarily is the resistance offered by the collateral arteries bypassing the obstructions. Run-off or "calf"resistance is found in the intramuscular arterioles, capillaries and venules and to a lesser extent in the veins draining the legs. In normal legs at rest the segmental resistance contributes only about 3-10 percent to the total extremity resistance; the remainder of the resistance is offered by the run-off vessels of muscle and skin (LUDBROOK 1966; STRANDNESS 1969; SUMNER 1977). Muscular exercise or temporary ischemia (e.g. by cuff occlusion) decreases the total resistance of the leg considerably to around 10 percent of the resting values. The effect is mainly attributable to dilatation of intramuscular arterioles, ihus markedly reducing the run-off resistance (LUDBROOK 1966; SUMNER 1977). The segmental resistance in healthy legs can be considered as more or less fixed and not to be influenced, whereas the callresistance is extremely variable and under the influence of sympathetic innervation, emotion, posture and locally produced metabolites that accumulate during exercise or ischemia (SUMNER 1977). The collaterals around obstructions which constitute the segmental resistance in blocked arterial segments are considered by some authors as "fixed" resistances, whereas others could find a small fall of this resistance during hyperemia (LUDBROOK 1966; SUMNER 1977). The increase of large vessel or segmental resistance in patients with arterial obstructions - which is directly related to the severity of stenotic atherosclerosis (FARRAR et al 1977) - is greatly compensated for by a reduction of the run-off resistance (KEITZER et al 1965; LUDBROOK 1966; SUMNER 1977). This explains why resting blood flow levels are equal in normal and diseased limbs. The drop of total peripheral resistance caused by exercise or induction of ischemia is less in legs with arterial. obstructive disease than in 
normal limbs (about $40-70$ percent as compared to 90 percent in healthy legs) (LUDBROOK 1966).

\subsection{INFLUENCES OF VESSEL GEOMETRY ON PULSATILE PRESSURE AND FLOW COMPLEXES}

\subsubsection{Normal vessel geometry}

The normal arterial tree is a conduit system containing a wide variety of geometrical configurations which all by themselves are most interesting from a hemodynamic point of view as they are accompanied by disturbances of flow profiles and may act as sources of fluid energy loss. Among them are tapering, curvatures, angulations, bifurcations and junctions, transition from circular to elliptical cross-section and tortuosity. A good survey of their hemodynamics, including many literature references, is to be found in the monograph "Hemodynamics for surgeons" by STRANDNESS and SUMNER, (1975). As indicated by the negligible energy loss along the greater healthy arteries (section 3.1) these configurations do not contribute much to energy losses. Their influence on the shape of the normal pressure and flow pulse waves was discussed in previous sections (sections 3.2 and 3.3.2).

Two abnormalities of vessel geometry are of practical importance to the vascular surgeon as they constitute the majority of lesions he is dealing with: obstructions, mainly of atherosclerotic origin, and aneurysms. Their hemodynamic implications will be discussed below.

\subsubsection{Stenosis}

A stenosis is a more or less abrupt decrease of the cross-sectional area of an artery occupying some length of that artery followed by a more or less abrupt increase of luminal cross-sectional area.

\subsubsection{Fluid energy losses due to stenosis}

The blood passing from an unobstructed artery into the narrowed segment is forced to accelerate which means an increase of kinetic energy. This is associated with a drop in potential energy or pressure and some loss of energy as heat (section 3.1). At the distal end of the stenosis where the lumen again widens, kinetic energy is transformed back into potential energy and the pressure rises, though not to its pre-stenotic level. A third factor contributing to the loss of energy is the viscous loss in the stenotic segment due to the high flow velocities at that site. Figure 3.3 gives a schematic and simplified representation of the three mechanisms reponsible for the loss of fluid energy casu quo pressure. The figure applies only to the situation of steady flow through an ideal stenosis. The viscous energy or pressure losses within the stenosis are small compared to those caused by the sudden contraction (entrance effect) and even smaller in comparison to the losses due to the sudden expansion of the vessel lumen (exit effect). (STRANDNESS and SUMNER 1975, chapter 6; SUMNER 1977).

For oscillatory flow through stenoses the matter is much more complicated because flow reversal and nubulence develop especially at the exit of the stenosis which is accompanied by extra energy losses (STRANDNESS and SUMNER 1975, chapter 6; FLANIGAN et al 1977). MAY et al (1963) and later 


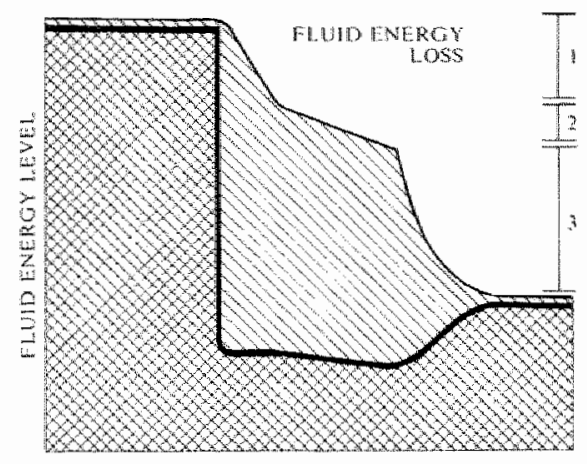

FIGURE 3.3

Energy losses of blood flow through a stenosis.

The total fluid energy level along the course of a constricted artery for steady flow is represented by the shaded area. The double shaded area represents the potential energy (= pressure) and the single shaded area represents kinetic energy. I = energy loss due to contraction of the vessel lumen, $2=$ viscous energy losses, $3=$ energy loss due to the sudden expansion of the vessel lumen. The heavy solid line represents the level of the intra arterial pressure along the artery.

(Redrawn from RUTHERFORD, R.B: "Vascular surgery", W.B. Saunders Company, Philadelphia, London, Toronto, 1977, Figure 4.6.).

YOUNG et al (1977) and FLANIGAN et al (1977) were able to formulate nearly identical mathematical equations which allowed to predict the pressure drop obtained across experimental stenoses in animal studies. The degree of pressure drop in these equations is related to the severity of the stenosis and the flow velocity in the prestenotic artery. The higher the prestenotic flow velocity is, the greater the pressure drop will be.

The differences in peak flow velocities in systole and in diastole explain why poststenotic systolic blood pressures are more reduced than mean or diastolic pressures (MAY et al 1963; FLANIGAN et al 1977).

If in an experimental setting the pressure drop across stenoses of variable severity can be kept constant, the flow through the stenoses diminishes with increasing luminal reduction. The exponential curve representing this relationship is an identically shaped mirror image of the curve which relates the pressure drop to the degree of stenosis at constant flow rates (Figure 3.4) (MAY et al 1963; STRANDNESS and SUMNER 1975; SUMNER 1977; FLANIGAN et al 1977).

Under natural circumstances stenotic lesions do not exist in isolation but constitute the "fixed" part of a variable hemodynamic system also comprising collaterals and the peripheral outflow bed. As mentioned in section 3.4, the increased segmental resistance in the larger stenotic vessels is compensated for by a reduction of the outflow resistance which in turn enhances the flow (velocity) through the stenosis. Up to a certain degree of stenosis this "vascular bed reserve (YOUNG et al 1977), "can keep the volume flow through the stenosed arterial segment constant, but further diameter reduction will lead to a lall in now and flow velocity.

\subsubsection{Critical stenosis, length of stenosis and multiple stenoses}

A "critical stenosis" is defined as the percentage by which the cross-sectional area of a vessel must be reduced in order to produce a measurable drop in blood flow and pressure or as the stenosis for which a further increase in the severity will cause a significant reduction in blood flow and pressure (MAY et al 1963; 


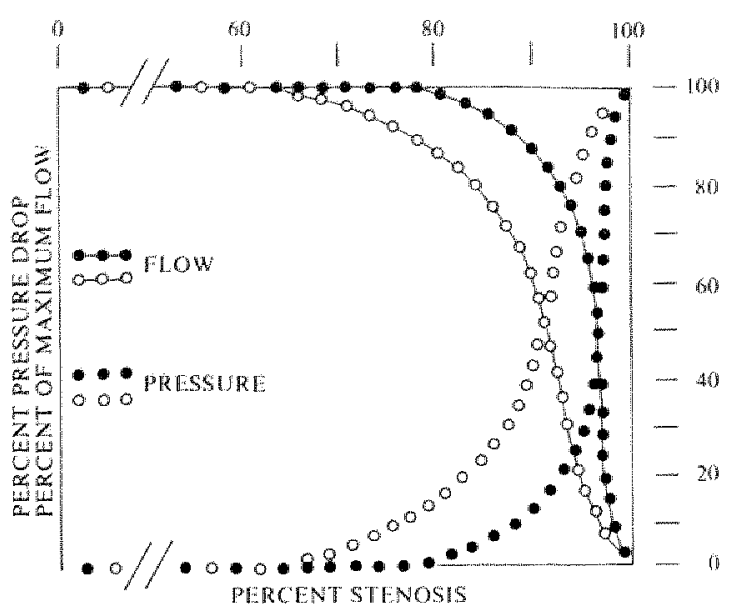

FIGURE 3.4

Critical stenosis curves.

The figure shows the relationship between the percentages of pressure fall (interrupted curves) and flow reduction (drawn curves) and percentage of stenosis (cross sectional area reduction). Curves are given for resting flow conditions in dots and for a condition of accelerated flow in circles. It should be noticed that the figure represents an experimental situation in which flow rate was artificially maintained at a constant level during determination of the pressure drops whereas pressure drops across the various stenoses were kept constant during determination of the percentages of flow reduction. (Adapted and redrawn from RUTHERFORD, R.B.: "Vascular surgery", W.B. Saunders Company, Philadelphia, London, Toronto, 1977, Figure 4.7.).

STRANDNESS and SUMNER 1975, p. 108; FLANIGAN et al 1977, YOUNG et al 1977).

The curves of Figure 3.4 are therefore sometimes referred to as "critical stenosis curves". They show that the critical area is the same for flow and pressure. However, a fixed critical stenosis does not exist because the breaking point depends on the flow velocity. If this increases, for example by peripheral vasodilatation due to exercise, the critical area of Figure 3.4 shifts to the side of lower stenosis percentages. At the same time the curves get a less sharp inflection which makes it difficult to determine the corresponding critical area (FLANIGAN et al 1977). Even for systolic and diastolic flow in the same heart beat the stenosis is critical at a different percentage. Calling a certain value of a stenosis "critical" is meaningless unless the flow velocity circumstances - which are primarily determined by the resistance of the vascular bed distal to the stenosis - are specified. Further determinants of the critical character of a stenosis are its length and the degree of collateral flow around it (YOUNG et al 1977). It has, therefore, been proposed to grade the severity of a stenosis after its effect on maximum flow rather than on the resting flow. For this purpose the term "vascular bed reserve" was introduced which is defined as the quotient of maximal flow to a peripheral bed over the resting flow (YOUNG et al 1977).

The so-called critical stenosis for various organ systems or tissue beds will be different as their normal resting flow velocities and vascular bed reserves are different. For the arteries to the leg the critical stenosis percentage under basa] resting conditions is generally accepted to be around $75-80$ percent cross- 
sectional area reduction. (HAIMOVICI and ESCHER 1955; MAY et al 1963; STRANDNESS and SUMNER 1975; SUMNER 1977). Under conditions of exercise so called "subcritical stenoses" far below this percentage may cause circulatory deprevation (SCHULTZ et al 1967; STRANDNESS and SUMNER 1975, p. 116). Changes in body position from head-up to head-down did not after the critical behaviour of iliac stenoses in animals (MAY et al 1963).

The length of a stenosis is of relatively minor importance for the energy loss across it. It does not affect the important entrance and exit effects and only at a minor scale the viscous losses (section 3.5.2.1). KINDT and YOUMANS (1969) determined the relation between stenosis percentage, length of the stenosis and the resulting proportional reduction of llow. Below 65 percent diameter stenosis its length bardly influenced the flow. In the range of 70-85 percent stenosis the influence of stenosis length on flow reduction was quite important, e.g. extending a 80 percent constriction from $2 \mathrm{~cm}$ length to $4 \mathrm{~cm}$ caused a flow reduction of 30 percent. This dependency of flow reduction on the stricture length was most marked in the shorter stenoses; further flow reductions with increasing length became smaller above $4 \mathrm{~cm}$ length of stenosis. At high stenosis percentages, short constrictions already caused such considerable flow reductions that any increase of the length hardly further reduced the flow. This means that the length of a stenosis has its most marked effect in a narrow stenosispercentage range around 75 percent diameter stenosis (MAY et al 1963; KINDT and YOUMANS 1969; STRANDNESS and SUMNER 1975).

The effect of multiple stenoses in a single unbranched artery is not a simple addition of the effects which each stenosis would have if isolated. In the case of identical stenoses each additional stenosis has less an effect on distal blood pressure than each previous stenosis (FLANIGAN et al 1977) When two stenoses of unequal diameter are in series the tighter of the two determines the resistance of the segment. The sequence of the constrictions is of no importance. If a so called "critical" and a "subcritical" stenosis are in series the effect on pressure and flow is almost entirely attributable to the critical stenosis (STRANDNESS and SUMNER 1975; SUMNER 1977; FLANIGAN et al 1977). Evidence has been produced that multiple so-called subcritical stenoses in series may produce a hemodynamically significant decrease in poststenotic pressure and flow (FLANIGAN et al 1977). If a collateral branch goes of between sequential stenoses these principles are no longer valid (STRANDNESS and SUMNER 1975, chapter 6; SUMNER 1977). The peripheral effects of the multiple stenoses is then mainly determined by the collateral capacity of that branch (BLAISDELL and GAUDER 1961).

\subsubsection{Pressure and flow pulse wave changes due to stenosis}

The pressure pulse waves and the flow pulse waves distal to stenotic lesions, as recorded through an intraarterial needle connected to a pressure transducer and an electro-magnetic flowmeter respectively, show characteristic features. The differences with the normal waves (sections 3.2 and 3.3.2) are a result of the stenosis itself and to a high degree also of the state of the peripheral outflow bed

* The relation between percentage cross-sectional area redtetion (A) and percentage dianeter reduction (D) for an ixisymmetric stenosis is given by equation $A=\left(2-\frac{D}{16}\right)$. D See also SPENCER and REID (1979). A cross-sectional area reduction of 75 perent thus corresponds to a diameter reduction of 50 percent. 
which usually has a lower resistance or impedance in case of proximal stenosis (section 3.4).

The poststenotic pressure pulse wave is damped, the peaks become rounded, the upslope is delayed, any notches on the downslope disappear and the downstroke becomes bowed away from the baseline. The pulse pressure is markedly reduced contrary to the mean pressure level which only shows a moderate decrease as long as the vessel is not totally occluded (BLAISDELL and GAUDER 1961; KEITZER et al 1965; HAIMOVICI and ESCHER 1975; BOLLINGER et al 1976; SUMNER 1977; HEINTZ et al 1978). If distal to the pressure monitoring site a second constriction is applied the pulse pressure and mean pressure level may be restored to normal unless a major collateral sidebranch goes of in between (BLAISDELL and GAUDER 1961; KEITZER et al 1965).

In the same way the poststenotic flow pulse wave is characterized by a roundening and more gradual rise and decline of the systolic peak as well as an attenuation of the amplitude. The most important feature is the disappearance of the reverse flow phase due to the fall in peripheral resistance. The mean flow as a result is less influenced. Occasionally a d.c. flow component appears throughout the cardiac cycle (KEITZER et al 1965; DEAN and YAO 1.976; YOUNG et al 1977; SUMNER 1977; INOKUCHI 1979).

\subsubsection{Aneurysms}

Hardly any published data are available about the hemodynamic effect of fusiform and saccular atherosclerotic aneurysms on peripheral pressure and flow. Most of these aneurysms contain laminated mural thrombi thick enough to leave only a residual lumen of approximately normal caliber through the aneurysm (SPITTELL and WALLACE 1980). The deposit of these thrombi is likely to be caused by boundary fluid layer separation at the entrance of the luminal expansion. Thus, the llow disturbances caused by an expanding aneurysm at the same time counteract their origin.

Flow reversal along the wall of a femoral aneurysm was found by YAO and BERGAN (1974). BÖHMER et al (1977) described enhanced amplitudes of all components of the (Doppler) flow velocity pulse wave derived from the aneurysm itself and a subnormal amplitude in the vessels distal to the aneurysm. Apart from some slowing down of the blood flow due to the aneurysmatic increased vessel diameter the hemodynamic effects of an aneurysm on distal perfusion are probably rather small (STRANDNESS and SUMNER 1975, chapter $6)$. 


\section{ULTRASONIC DOPPLER BLOOD FLOW VELOCIMETRY}

\section{1. PRINCIPLES OF ULTRASONIC DOPPLER FLOW VELOCITY DETECTION}

During its passage through body tissues ultrasound is absorbed at an approximate rate of $1 \mathrm{~dB} / \mathrm{cm} / \mathrm{MHz}$. However, the absorption varies substantially from tissue to tissue. In blood, for instance, the absorption rate is much lower then in bony tissue (WELLS 1974a). Apart from absorption, two other factors influence the course of the ultrasound waves: reflection by large surfaces and re-radiation by small elastic particles. Whether a particle is "small" depends on its size relative to the sound wavelength. Erythrocytes, for instance, with a largest diameter of about $8.10^{-3} \mathrm{~mm}$ are small when compared to a wavelength of $0.3 \mathrm{~mm}$ of a sound beam of $5 \mathrm{MHz}$. Therefore, the blood cells behave like elastic point scatterers for the incident ultrasound (FLAX et al 1974; GOSLING 1976). The degree of reflection from tissue boundaries depends on the ratio of the acoustic impedances of the materials on either side of the boundary. It is high for air/tissue and soft tissue/bone interfaces. Considerable reflection may occur from the blood vessel wall/blood boundary. The intensity or amplitude of the sound waves reflected from such boundaries is considerably higher than that of the sound backscattered from blood cells (MAURIZI 1974; GOSLING 1976).

When the reflecting interface or scatterer is moving, the returning ultrasound is shifted in frequency from the incident wave. This effect is known as the Doppler effect after the Austrian mathematician and physicist Christian DOPPLER (1803-1853), who first described the phenomenon for star-light in 1842. In 1845 the Dutch physicist Christophorus BUYS BALLOT (1817-1890) could prove that the "Doppler" effect also occurred with sound waves. In cases where the source of the emitted sound and the receiver for reflected waves are located at the same place, the observable Doppler frequency shift $(\Delta f)$ is given by the formula:

$$
\Delta \mathrm{f}=\frac{2 \cdot \mathrm{f}_{0} \cdot \mathrm{v} \cdot \cos \alpha}{\mathrm{c}}
$$

where $f_{b}$ is the frequency of the emitted sound beam, $v$ is the velocity of the moving scatterer or reflector, $\alpha$ is the angle between the incident sound beam and the direction of the movement and $c$ is the velocity of the sound in the insonated material (see Figure 4.1). If the movement is towards the source of the sound the frequency of the returning sound is increased (positive frequency shift) and if the movement is away from it, the reflected or backscattered sound becomes lower in lrequency (negative frequency shift).

Nowadays this Doppler effect is widely used in the transcutaneous, noninvasive, detection of blood flow vellocities. The ultrasonic frequencies usually employed in physiological tests for medical diagnosis are in the range from 1 to $10 \mathrm{MHz}$. The sound waves are of low intensity and hence biologically harmless (WELLS 1974a). The anatomic region where a blood vessel to be studied can be expected is insonated with a beam of ultrasonic waves directed at the moving targets (red blood cells) within that vessel. The backscattered sounds are 


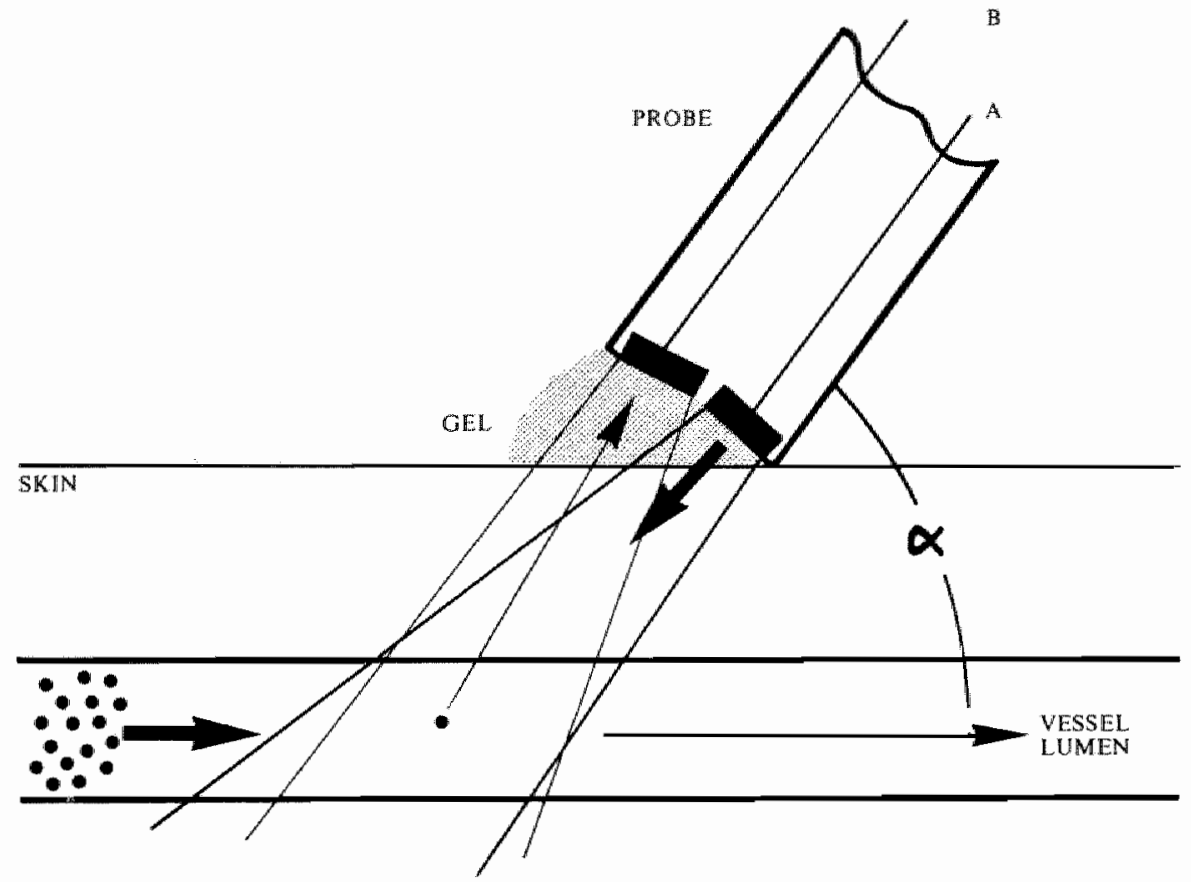

FIGURE 4.I

The principle of Doppler flow velocity determination.

$A$ is the ultrasound transmitting crystal, $B$ is the ultrasound receiving crystal, $\alpha$ is the angle between the flow direction (indicated by the arrow in the vessel lumen) and the transducer or probe axis. The dots in the lumen represent the red blood cells reflecting the incident sound waves emitted by A.

received with appropriate equipment. Ultrasound reflected from stationary tissue surfaces is not shifted in frequency. In contract, the ultrasound waves backscattered from the moving blood cells in the vessels are shifted in frequency by an amount proportional to the velocity of the cells (STRANDNESS et a 1966 ; ROBERTS 1976; GOSLING 1976; RENEMAN and HOEKS 1977; RENEMAN et al 1979). The received ultrasonic frequencies are compared with the frequency of the emitted sound, using various demodulation techniques. As a result of this weighing process, the Doppler frequency shifts are obtained. In the examination of bloodvessels these Doppler signals are usually in a range below $10 \mathrm{KHz}$, if emission frequencies of up to $10 \mathrm{MHz}$ are employed. Such signals are perceivable by the human ear if properly amplified. They further may be used in different processing methods as will be discussed in section 4.5. In principle the Doppler frequency shift detection allows the determination of the velocity of the insonated red blood cells.

The above described behaviour of the ultrasound waves explains the limitations for ultrasound Doppler examinations. Air/tissue boundaries reflect nearly all soundwaves. This makes the examination of blood vessels positioned behind air-containing organs, like lungs or bowels, difficult or impossible. The same applies to vessels hidden behind bony structures like the skull. A good, airfree, acoustic coupling between the ulltrasound probe and the insonated tissues 
is mandatory. This is usually accomplished by maintaining a layer of watery gel between the surface of the probe and the skin (see Figure 4.1). Another limitation set to Doppler examinations is the depth of sound penetration into the tissues. Both the amount of backscatter and the quantity of absorption, and thus the attenuation of the incident sound beam, increases at higher transmitter frequencies, causing a relatively shallow tissue penetration (WELLS 1974a; GOSLING 1976; RENEMAN and HOEKS 1977; RENEMAN et al 1979). For the detection of low flow velocities a high transmitter frequency is necessary as will be evident from the Doppler formula. This conflict between the optimal frequency for a good Doppler shift and a deep tissue penetration necessitates a compromise choice of the frequency (MAURIZI 1974; RENEMAN and HOEKS 1977). For peripheral arteries of the leg frequencies up to $10 \mathrm{MHz}$ are used. whereas for the study of deeper situated vessels like the aorta or the iliac vessels 5 $\mathrm{MHz}$ or less is required (WELLS 1974b; YAO and BERGAN 1974; GOSLING 1976).

\subsection{DOPPLER BLOOD FLOW VELOCITY DETECTORS}

A great choice of instruments to detect the Doppler shift due to the blood flow is commercially available. The devices are often referred to as Doppler flow meters or simply Doppler meters. Most Doppler meters not only contain the circuitry to detect the Doppler frequency shift but also a part which processes the Doppler signal to a form suitable for further analysis. Doppler meters may have the capacity to distinguish between positive and negative Doppler shifts and are then called directional meters. If they lack this feature they are of the non-directional type. According to their mode of operation continuous wave (CW) and pullsed Doppler (PD) systems can be distinguished.

\subsubsection{Continuous wave (CW) Doppler systems}

At present the continuous wave (CW) system is the most widely used type of Doppler device. In these systems an ultrasonic sound beam is produced by continuously exciting a piezoelectric transducer crystal at the desired megahertz frequency. The back-scattered ultrasound is continuously received by another identical piezoelectric crystal, mounted adjacent to the transmitting crystal in the same transducer or probe (ROBERTS 1976; RENEMAN and HOEKS 1977; RENEMAN et al 1979) (see Figure 4.1). The CW Doppler meters have the advantage of being easy to build and to operate (RENEMAN and HOEKS 1977; RENEMAN et al 1979). Two disadvantages are inherent to the CW technique. At the first place the ultrasound $C W$ beam, which has a width of a few millimeters and penetrates the tissues for some centimeters, may not only insonate the blood vessel under examination but also other vessels in the neighbourhood (see Figures 4.2 .2 and 4.2.4). This causes Doppler shift frequencies which may be misinterpreted as originating from the vessel at which the beam purposely was directed. Another problem is that of lateral vessel wall movements within the ultrasound beam due to the expansile nature of the pulsations. These cause lowfrequency Doppler sounds of high amplitude. The intensity (or power) of these signals is approximately 30 times higher than that of the signals induced by the moving blood cells (GOSLING 1976; RENEMAN and HOEKS 1977; RENEMAN et al 1979; RENEMAN 1981). These vessel wall artifacts can mask the presence of high-frequency components in the Doppler signal. In the common CW devices, 
therefore, frequencies below $200 \mathrm{~Hz}$ often are eliminated by filtering the signal which however causes a loss of low-frequency (equivalent with low velocity) information (STRANDNESS et al 1966).

\subsubsection{Pulsed Doppler (PD) meters}

In pulsed Doppler (PD) systems a transducer crystal emits at a high repetition frequency bursts of ultrasound. During the intervals between two pulses the same crystal receives the returning soundwaves. The limited time when the crystal operates in the receiving mode is dictated by an electronic gate (ROBERTS 1976; WOODCOCK 1977; RENEMAN and HOEKS 1977; RENEMAN et al 1979; RENEMAN 1981). Thus only Doppler signals generated at a specific distance from the transducer are received. The $\mathrm{PD}$ combines the action of an echo sounder and a flow velocity detector and takes, as it were, small-volume Doppler samples from the insonated region. Usually the so-called single-gate PD systems allow the selection of various time delays by which Doppler signals can be sampled from various depths. Sometimes the gate-width is also variable. These characteristics of the PD meters make it possible to assess the vessel diameter and give insight in the distribution of blood flow velocities across the vessel diameter and hence of the flow velocity profiles (section 3.3.3.) (DEAN and YAO 1976; RENEMAN and HOEKS 1977; RENEMAN et al 1979). Although this in principle would permit noninvasive volume flow measurements, this ideal is not yet reached due to errors inherent to the circuitry, which make the determination of the vessel diameter inaccurate (RENEMAN et al 1979). The main disadvantage of $\mathrm{PD}$ systems is that the maximum velocity which can be detected is limited, among other things by the repetition frequency of the pulses (WOODCOCK 1977; RENEMAN and HOEKS 1977; HOEKS et al 1981). Another drawback of the single-gate PD meters is the difficulty to find the blood vessels with them (RENEMAN et al 1979).

Recently multi-channel PD systems have been developed with the ability to analyze, simultaneously and instantaneously, velocities in adjacent volume samples. With these systems the velocity profile can be recorded on-line at discrete time intervals during a few cardiac cycles (PERONNEAU et al 1974; MCLEOD 1974; HOEKS et al 1981). In the present generation of these systems samples can be taken along the vessel diameter at distances of $0.5 \mathrm{~mm}$ (RENEMAN $\llbracket 981$; HOEKS 1982).

\subsubsection{Directional information}

In the sections 3.3.3. 3.3.4. and 3.5.2. the presence of flow in opposing directions during part of the cycle was discussed as it exists in the larger arteries of the extremities. The importance in hemodynamic studies of simultaneously measuring the co-existing two-directional flow velocities may follow from it. Doppler systems which exhibit the feature of reliably resolving such mixed velocities are called directional systems. Both $\mathrm{CW}$ and PD systems can be of the directional type. Non-directional meters are still frequently used in studies where merely the presence or absence of flow should be established, like in segmental systolic pressure measurements (to be discussed in section 5.1 ). The separation of positive Doppler frequency shifts from negative ones can be obtained by three different techniques presently incorporated into commercially available equipment. These techniques, as summarized by GOSLING 
(1976), WOODCOCK (1977) and GOGHLAN and TAYLOR (1978), are the single side-band demodulation technique, heterodyne techniques and phase-rotation or phase-quadrature techniques (see also NIPPA et al 1975). The principles of these methods of detection of flow direction and references giving access to detailed information about the electronics involved, can be found in the mentioned publications. A more extensive discussion of the techniques is considered to be outside the scope of this studly.

\subsection{THE DOPPLER FREQUENCY SPECTRUM}

The red blood cells at various sites in a transverse section of a blood vessel are not moving all at the same speed (section 3.3.3). Therefore, the Doppler signal caused by the movement of these erythrocytes does not contain one single frequency but a spectrum of frequencies, the so-called Doppler frequency spectrum. This spectrum is then available for further processing (to be discussed in section 4.5.). Apart from this - most important - cause of the occurrence of a frequency spectrum in Doppler velocimetry, some instrument related factors are responsible for a broadening of the band of frequencies received (WELLS 1974b; RENEMAN and HOEKS 1977;BLACKSHEAR et al 1979; RENEMAN et al 1979). The divergence and dimensions of the ultrasound beam cause minor variations in the incident Doppler angle between red blood cells at different points along the course of the vessel, which in turn causes variations in the frequency of the reflected sound beam even for red blood cells moving at the same speed. Also the emitted ultrasound spectrum contains more than just one central frequency. This explains why in pulsed Doppler examinations of stable flows, when the velocity of most erythrocytes within the sample volume are nearly identical a band of Doppler frequencies rather than a single discrete frequency is present (BLACKSHEAR et al 1979).

\subsection{FACTORS INFLUENCING THE DOPPLER FREQUENCY SPECTRUM}

The spectrum of Doppler frequencies is in the first place determined by the elements constituting the Doppler formula as given in section 4.1 . of which the flow velocity (v) of the blood cells is the most important one and the variable usually sought for in the examinations. Increasing the emission frequency of the Doppler meter, while leaving all other circumstances the same, will broaden the range of frequencies present in the received Doppler signal. Also alterations of the angle between the sound beam and the main direction of the blood stream will change the Doppler frequency spectrum. For each insonation frequency a beam/vessel angulation exists which gives the highest frequencies in the spectrum (ROBERTS 1976). Higher frequencies will appear in the spectrum when the angle approaches to zero. However, this is in conflict with the requirement of maintaining a good skin contact and probe/vessel distance. Practically, the optimum angle between the probe and the vessel is between 30 and 60 degrees (RAM and BHMAN1 1971; JOHNSTON et al 1977b). Theoretically, the Doppler spectrum ought to disappear when the ultrasound beam is perpendicular to the vessel axis since the cosine of $90^{\circ}=0$. Due to beam divergence and the dimensions of the beam (section $4.3_{\text {. }}$ ) as well as the fact that not all red blood cells move parallel to the vessel axis, a Doppler spectrum will be received even when the vessel is insonated perpendicularly. It could be shown experimentally that over a wide scale of erythrocyte concentrations (Hemato- 

1971).

Because the receiving and the transmitting crystal in CW Doppler probes are usually placed under an angle of $20-30^{\circ}$ with respect to each other (WELLS $1974 \mathrm{~b}$ ), the receiving and transmitting beam axes converge and give a narrow area of overlap of both beams (see Figures 4.1. and 4.2.). Outside this focussed

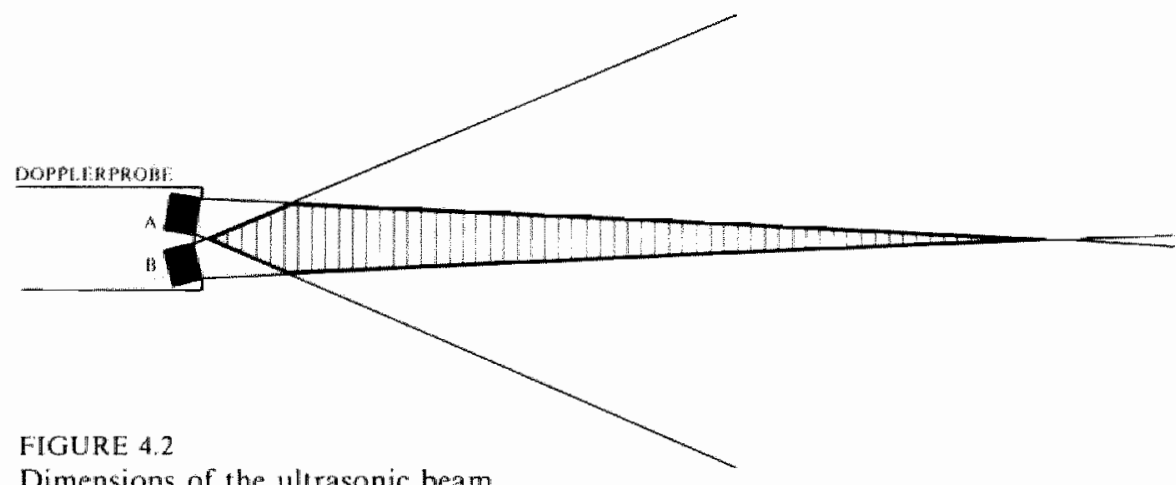

Dimensions of the ultrasonic beam.

$A$ and $B$ represent the emitting and receiving crystals mounted in the Doppler probe. Only the area of overlap (shaded) of the receiving and transmitting beam is effective in Doppler measurements. The length and width of the beam is determined by the emission frequency, the convergence angle of the crystals and the distance between the crystals. The distance from the probe to the place where the beam has its greatest width is called the focus distance of the probe.

area the signals are severely attenuated and contribute less to the Doppler spectrum. The overlapping region can be considered for practical purposes as "the ultrasonic beam". The distance from where this "beam" has its greatest diameter to the probe is called the focus distance of that probe. Depending on the distance between the transducer and the blood vessel under examination and/or the relation between the width of the focussed beam and the vessel diameter various Doppler frequency spectra may be obtained from the same vessel. (Figure 4.3.).

For instance, if the beam is narrow compared to the vessel lumen the Doppler spectrum will contain high frequencies if the beam intersects the vessel axis (Figure $4.3^{2}$ ) and mainly lower frequencies if only the periphery of the luminal cross-section is insonated (Figure $4.3^{3}$ ) (WOODCOCK et al 1972 b; RENEMAN and SPENCER 1974; ROBERTS 1976; RENEMAN and HOEKS 1977; RENEMAN et al 1979).

\subsection{METHODS OF DOPPLER AUDIOSIGNAL PROCESSING}

The type of data pursued in Doppler examinations may range from the mere establishment of the presence of flow to information about the instantaneous velocity profile in a vessel. Often the investigator is interested in the instantaneous maximum or instantaneous mean frequency of the signal as a representation of the maximum or the mean of the blood flow velocity in the examined blood vessel respectively. The processing techniques to obtain this information from the Doppler signal will be discussed in the following sections. 

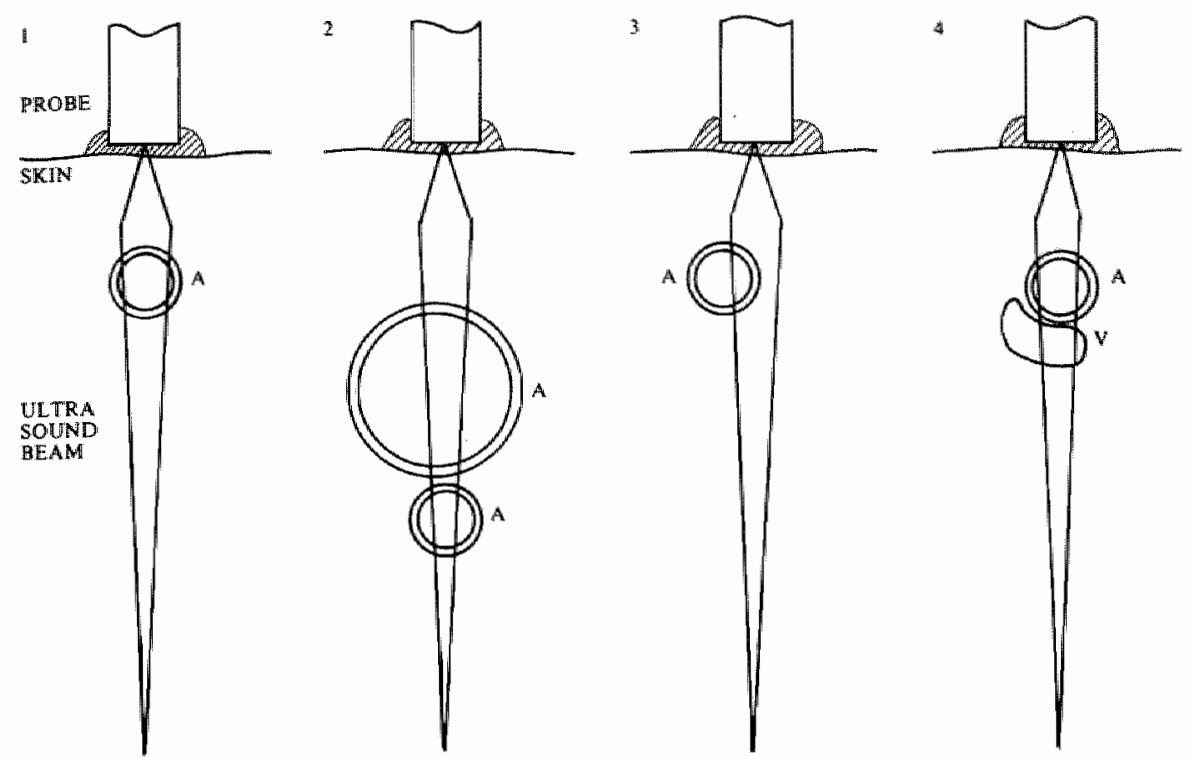

FIGURE 4.3

Various situations of continuous wave ultrasound beam/vessel intersection.

The probe is coupled to the skin by a gel.

$A=$ cross section of an artery, $V=$ cross section of a vein.

1. Artery cross section fully insonated by ultrasound beam.

2. Wide artery and adjacent side branch both partially insonated. Frequencies from both arteries will be present in the Doppler spectrum.

3. Artery only partially insonated. Only low frequenches generated at the periphery of the lumen, where flow velocities are low, will appear in the Doppler spectrum.

4. Simultaneous insonation of an artery and a vein, causing mixed frequencies in the Doppler spectrum.

\subsubsection{Acoustic presentation}

Amplification of the Doppler signal allows the study of its pitch (frequency) and loudness (amplitude). The sound can be heard from earphones or loudspeakers. By the use of stereophonic equipment directional information in the original Doppler signal can be preserved. This most simple way of processing the Doppler signal is mainly used in systolic blood pressure measurements (to be described in section 5.1.). Other applications will be described in section 6.2.1.

\subsubsection{Zero Crossing Detection (ZCD)}

Certainly the most common method currently used for determination of the average frequency of the Doppler spectrum and conversion of this spectrum into a visible signal is by means of the so-called zero crossing detector ( $Z C D)$. The composite mixture of Doppler frequencies fed into this device - which under limited circumstances can be considered a frequency to voltage converter - is reduced to a single analogue voltage. The output, which is related but certainly not equal to the instantaneous mean frequency of the introduced Doppler 
frequency spectrum, can be visualized on an oscilloscope or strip chart recorder. The method has the advantage of being inexpensive and giving on-line a signal which is easily understood by technicians and physicians. It certainly is sufficient for simple velocity pattern studies of the type to be discussed in section 6.2.2. A great disadvantage in more profound Doppler signalanalysis is the fact that the output of the $Z C D$ is far from a true representation of the average flow velocity across the vessel (RENEMAN and SPENCER 1974; ROBERTS 1976; RENEMAN and HOEKS 1977; JOHNSTON et al 1977b). In animal experiments it could be shown that the instantaneous flow velocity tracings from a Doppler/ ZCD meter were similar to the tracings obtained with an electromagnetic flow meter under resting conditions. At peak flow conditions high-frequency information was lost with the ZCD processing as demonstrated by a flattening of the curwes (RENEMAN et al 1973; RENEMAN and SPENCER 1974). A ZCD only represents the average velocity across a vessel if the velocity profile is an ideal parabolic one with evenly distributed scatterers and the ultrasound beam bathes the whole cross sectional area of the lumen. Under these theoretical and nonphysiological conditions the ZCD overestimates the mean frequency (velocity) with about 15 percent (WOODCOCK et al 1972b; ROBERTS 1976; RENEMAN and HOEKS 1977; WOODCOCK 1977b). With the normal pulsatile change of the velocity profile much greater errors in the mean frequency (velocity) determination occur (ROBERTS 1976; JOHNSTON et al 1977b). Other regularly reported disadvantages of the ZCD are the dependency of its output of the amplitude of the Doppler signal. At high signal intensity this is not a great problem, but with low amplitude sounds the poor signal-to-noise ratio becomes an important factor in the distortion of the output (FLAX et al 1974; RENEMAN and SPENCER 1974; ROBERTS 1976; WOODCOCK 1977; RENEMAN and HOEKS 1977; JOHNSTON et al 1977b). Also the high amplitude-low frequency components of the Doppler signal due to wall movements ("wall thumps") are seriously disfiguring the analogue trace produced by the ZCD (GOSLING and KING 1974b; RENEMAN et al 1973; RENEMAN and SPENCER 1974; JOHNSTON et al 1977b). Other disadvantages of the ZCD for the processing of Doppler sound were discussed by the group of Gosling: the interference of radio-frequencies with the signal; the impossibility to distinguish from each other signal components caused by the intended insonation of an artery and the accidental inclusion of a neigh bouring vein in the sound beam (see Figure $4.3^{4}$ ); and the damping of the output signal due to the averaging process within the ZCD, causing loss of information on transient velocity changes (GOSLING and KING 1974b). As a result of the mentioned drawbacks of the ZCD in Doppler signals processing, the change is real that distorted waveforms may be generated and interpreted physiologically, since the artifacts may not be self-evident (GOSLING 1976).

\subsubsection{Audio frequency spectrum analysis}

According to GOSLING (1976) an ideal processing and displaying system for Doppler shift data should have the ability

- to display all frequencies present at any instant;

- to indicate the amplitude of such signals over the entire frequency range;

- to display both positive and negative Doppler shifts simultaneously;

- to perform its task over any required length of time and in real time.

These demands are met by a variety of commercially available audio frequency 
spectrum analyzers. The instruments are capable of presenting all the frequencies present in the Doppler audio spectrum in smaller or wider contiguous frequency steps versus either the power or the amplitude of each of those frequency steps. In real-time spectrum analyzers this is accomplished by time compression analysis techniques or some variant of digital Fast Fourier Transform (FFT) algorithm. Each scanning or "sweep" through the frequency spectrum takes only a few milliseconds and is repeated immediately after the previous one. In this way the Doppler signal is continuously sampled by the successive analysis sweeps. The number of samples which can be analyzed per second depends on the instrument characteristics and on the selected width of the frequency steps within the sweep and may amount to over $200 /$ second. The analy sis results of each of these sweeps can then be displayed on a CRT screen on as a hard copy by means of an UV recorder. In this way a three dimensional picture can be developed, usually with time along one axis (x-axis), the frequency along the other axis ( $y$-axis) and the amplitude of each of the small frequency steps either as lateral deviations from the sweep line or as variations in the intensity or gray scale of the display ( 2 -axis). The resulting display often is referred to as a sonagram or spectrogram (WOODCOCK et al 1972b; DEAN and YAO 1976; GOSLING 1976; RENEMAN and SPENCER 1979). An example of a sonagram is given in Figure 4.4.

FREQUENCY (KH)

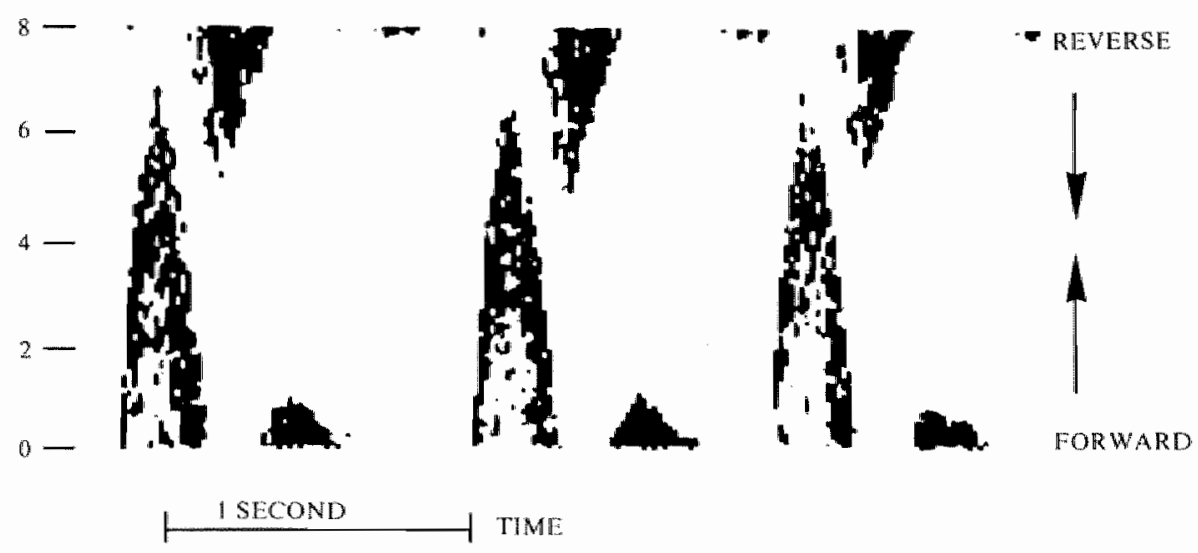

FIOURE 4.4

Sonagram of a Doppler signal.

Example of a sonagraphic display or "sonagram" of a directional Doppler signal of a normal common femoral artery, displaying three successive heart beats. Time is along the horizontal axis, frequency along the vertical axis and the amplitudes of the various frequencies are indicated by the gray scale of the figure. Notice the "window" of low amplitude frequencies in the first (systolic) forward component of the signal.

It is possible to obtain some information about the velocity profile in a vessel from the sonagram. If there is an even distribution of the signal amplitude over the displayed frequencies the profile must be parabolic. If the velocity profile is flat, only a narrow frequency band will show-up in the sonagram. With turbulence the spectrum outline becomes irregular and tattered, the normal 
windows of low or zero-amplitude frequencies in the systolic peaks (see Figure 4.4.) disappear and the spectrum broadens (WOODCOCK et al $1972 \mathrm{~b}$; ROBERTS 1976; RENEMAN and SPENCER 1979).

The practical advantages of a sonagraphic display of the Doppler data may be summarized as follows:

- The system has a favourable signal-tomoise ratio because noise and artifacts usually have an intensity or grayscale and temporal distribution which differ from the velocity signals (GOSLING 1976; ROBERTS 1976; WOODCOCK 1977).

- Differentiation is possible between the velocities detected from adjacent vessels in CW examinations (ROBERTS 1976; WOODCOCK 1977).

- The display process has no significant time constants (filtering which might distort the velocity-versus-time waveform and thus all time intervals are preserved in true register (GOSLING 1976).

The two main disadvantages of spectrographic analysis are the high purchase and running costs of the equipment and the fact that additional processing equipment is required to determine on-line the instantaneous average or maximum frequency of the spectrum.

For the sake of completeness a promising other technique should be mentioned, which more simply would give this information directly, namely the analogue Doppler signal display with the aid of a Phase-Locked-Loop (PLL) technique (ROBERTS 1976; SAINZ et al 1976, 1977). This technique makes it possible to detect continuously the peak frequencies and the minimum frequencies of the Doppler spectrum and display them as a simple analogue trace. It possibly could also be used to obtain an approximation of the instantaneous mean frequency of the signal. The electronic components needed for such a PLL analysis are low priced and readily available. 


\section{SEGMENTAL SYSTOLIC PRESSURE MEASUREMENTS IN THE LEGS}

Arterial obstructions usually cause a disturbance of hemodynamics with loss of energy of the blood flow at the site of the obstruction. This results in an abnormal pressure fall across the affected vascular segment (section 3.5.2.1). Thus, a relative hypotension is to be found downstream from hemodynamically significant arterial obstructive lesions. The degree of hypotension can be measured indirectly with a sphygmomanometer and an appropriate flow detector, like a Doppler ultrasonic device or pulse sensing equipment. Measurements at various levels of the legs are possible and are called segmental blood pressure measurements.

In this chapter aspects of the methodology of segmental pressure measurements, including the use of hyperemia, will be considered. Normal values and values found at various clinical stages of obstructive arterial disease will be discussed as well as the relation between angiogr aphical findings and segmental blood pressures.

\subsection{METHODS OF MEASUREMENT}

Basically the indirect blood pressure measurement in the leg is performed in the same way as in the arm: distal to a sphygmomanometer cuff the disappearance and recovery of the blood flow is observed during inflation and deflation of the cuff. At the leg the pulses often are not palpable and Korotkoff's vessel wall sounds are seldomly perceivable. In the near past plethysmographic techniques were generally used to detect pulsations downstream from the sphygmomanometer cuff. Most of the present knowledge about segmental limb pressures was gathered with these techniques (STRANDNESS 1969). Only after the introduction of Doppler equipment for the transcutaneous detection of blood flow, the segmental blood pressure measurements in the legs could become a routine clinical examination. The usual Doppler apparatus only permits the detection of systolic blood pressures. This hardly is a disadvantage since obstructive lesions in the arteries are more sensitively reflected in changes of the systolic pressures than in changes of diastolic or mean pressures (section 3.5.2.1.). The pressures mentioned in the following sections are systolic arterial pressures. Pressures are usually determined during deflation of the cuff(THULESIUS 1971; BRUIJNINCKX 1976). The most common levell of measurement is at the ankle immediately proximal of the malleoli (ankle blood pressure). Measurements are also performed at various levels of the upper part of the leg (thigh pressures) or about the knee. The level at which the pressure is determined is given by the position of the cuff and not by that of the Doppler probe. In thigh pressure measurements as well as in ankle pressure measurements the probe can be placed at one of the ankle arteries (HYLKEMA 1975; GOSLING 1976; BRUIJNINCKX 1976). For correct measurements a cuff having a width equal to 120 percent of the diameter or 38 percent of the circumference of the limb at the place of measurement is recommended (KIRKENDALL et al 1967; GEDDES and TIVEY 1976). A cuff which is too narrow for the limb gives fallse-high pressure readings. In the measurement of the ankle pressure the standard arm-cuff (12 
crn width) is suitable (BRUIJNINCKX 1976). Generally, it hardly makes a difference whether the probe is placed at the anterior tibial (dorsalis pedis) artery or at the posterior tibial artery (CARTER 1969; BOLLINGER et al 1970). The measurements are well reproducible both in healthy people and in patients (SIGGAARD-ANDERSEN et al 1972; GRUNTZIG 1975; BRUIJNINCKX 1976; SMITH et al 1977). A good correlation exists between the indirect (cuff) ankle pressure values and direct intraarterial measurements (GRUNTZIG et al 1971; BOLLINGER et al 1976). However, the possibility of poorly compressible arteries resulting in apparently very high ankle blood pressure values should be kept in mind. In large series of patients this accurs in 0.6-1 percent of the cases (CARTER 1968; HOBBS et al 1974) and in patients suffering from advanced leg ischemia or gangrene even in 6 percent of the cases (THULESIUS 1971; THULESIUS and GJÖRES 1971). It mostly concerns patients with calcifying medial sclerosis in diabetes mellitus (RAINES et al 1976). Edema of the lower legs may be another cause of false-high pressure values (BOLLINGER et all 1976). In the determination of the thighpressure the chosen cuff-width is of critical importance. The intraarterial pressure in the common femoral artery normally is equal to the pressure in the brachial artery (PASCARELLI and BERTRAND 1964). In indirect pressure measurements high at the thigh this equality to armpressure is only achieved by the use of $17-19 \mathrm{~cm}$ broad cuffs (FARIS and JAMIESON 1975; HEINTZ et al 1978; HURLOW et al 1978). Sometimes - for instance for a more accurate identification of aortoiliac obstruction - narrow thigh cuffs are used on purpose, accepting the discrepancy between the intra-arterial pressure and the indirectly measured values (HEINTZ et al 1978). Determination of segmental limb pressure at the level of the calf of the knee is less frequently used and unnecessary for the answer to most clinical problems (CARTER 1968; SIGGAARD-ANDERSEN 1972; CUTAJAR et al 1973). In detailed studies of the lower leg vessels the combination of measurements at these levels with ankle pressure measurements may be useful (SIGGAARDANDERSEN 1972; BRUIJNINCKX 1976).

It is common practice to relate segmental leg pressures to the arm blood pressure. The mere determination of absolute leg pressures hardly allows comparison between individuals because of the differences in systemic blood pressures. Only in patients with ischemic necrosis or pre-gangrene knowledge of the absolute values of the distal perfusion pressure of the tissues of the limb is important (RAINES et al 1976). The segmental leg pressure may be expressed as a gradient between the arm and the leg pressure, as a percentage of the arm pressure or as the ratio of leg ower arm pressure (pressure index). Whether pressure gradients of pressure-indices are employed makes no difference for the usefulness of the results (BRUININCKX 1976; BUTH 1978; GAY and ROBERTS 1979). In literature, index values are more frequently used than gradients.

\subsection{THE USE OF HYPEREMIA IN PRESSURE MEASUREMENTS}

Muscular activity of an extremity accelerates the bloodflow in that limb. This effect continues for some period of time after stopping the exercise ("postexercise hyperemia"). Also the temporary interruption of arterial inflow of the limb - e.g. with an inflated bloodpressure cuff - induces a reactive ("postocclusion") hyperemia (HILLESTAD 1963a, 1963b, 1963c; MAHLER et al 1976). Under the influence of the increased blood flow velocity hemodynamic changes due to abnormalities of vessel geometry are better manifested than under resting 
conditions. Particularly, an (additional) blood pressure fall can be elicited distal to arterial stenoses or occlusions by the hyperemia. Muscular exercise may be performed while walking, preferably during a standardized walking test on a treadmill, or as an isolated leg exercise on a calf, foot, or thigh ergometer. In patients a walking test causes a more profound drop in distal leg pressures than isolated leg exercise of equal duration (BAKER 1978). An additional advantage of the walking test above isolated leg exercise is the opportunity to assess the degree of disability of the patient caused by claudication. It also gives an idea about the patient's general condition (cardiorespiratory and locomotory system). This information is essential if considering vascular surgery. $A$ disadvantage is the poor reproducibility of the walking test (BAKER 1978). The post-occlusion hyperemia is not influenced by the cooperation of the patient and therefore better reproducible. The test does not demand special equipment like a treadmill or ergometer. The degree of ankle pressure fall after a thigh occlusion of 7 minutes duration is equal to that caused by a walking test of 5 minutes both in normals and in patients (BAKER 1978). However, the duration of any pressure fall after the occlusion test is much shorter, mostly less than two minutes (BAKER 1978; HUMMEL et al 1978). This may interfere with a correct performance of repeated pressure measurements during the hyperemic phase. Although the hyperemic effects are increasing with occlusions of longer duration, five minutes are sufficient for normal clinical testing (HILLE STAD 1963a; EHRINGER 1971). After such a period of (thigh) occlusion no effects on systemic blood pressure were noticed after release of the cuffs (BRENER et al 1974). The more proximal at the leg an occluding cuff is applied, the more pronounced the hyperemic reaction will be (EHRINGER 1971). A combination of circulatory arrest and legexercise ("ischemic exercise") has been employed in calf blood flow studies but this approach was not more effective in provoking hyperemia than the occlusion alone (HILLESTAD 1963c). A study comparable to this last one but using pressure as a parameter of the changes in circulation could not be found. The magnitude and the duration of the peripheral pressure fall during hyperemia is generally considered to be a reflection of the severity of the obstruction and the degree of collateral bridging (DEAN and YAO 1976).

Based on the duration and the degree of the pressure drop various "pressure response curves" can be distinguished. Already in the sixties - still using the strain gauge as a pulse detector - fundamental studies were undertaken to find the relation between ankle pressure changes and treadmill exercises of increasing workloads in normals and in patients with atherosclerosis (STRANDNESS 1969; SUMNER and STRANDNESS 1969). Three types of abnormal ankle pressure responses were distinguished: In type I the pressure was unrecordable immediately after exercise but recovered within 2-6 minutes; in type II the pressure remained unrecordable for as long as 12 minutes, whereas in type III the recovery time lasted even longer. This classification was only based on the duration of the pressure fall. In all types the pressures apparently were unrecordable for some time. Maybe this was due to the technique of pressure measurement with a strain gauge placed around the toe. According to YAO usually four types of abnormal pressure response curves (or pressure patterns) determined with the Doppler method - are distinguished: In the type I curve there is no fall in pressure although the resting pressure may be below normal; in type II the pressure drops less than 50 percent and it returns to a pre-exercise level within 5 minutes; in type III the pressure fall exceeds 50 percent and it requires $5-15$ minutes to return to the pre-exercise value; in the type IV response 
the pressure becomes unrecordable for some time and it takes 15 minutes or more to reach the pre-exercise level (YAO 1970, 1973; LEWIS et al 1972a; LEWIS and YAO 1974: NAYMAN 1974; CHAMBERLAINet al 1975). A further extended classification in 9 classes of ank le pressure response curves was devised by $G A Y$ and ROBERTS. Their analysis of the pre-and post-exercise pressure values was computerized and was based on four main criteria: whether the resting ankle pressure was below or above the arm pressure; whether the maximum postexercise pressure was below or above resting pressure; the ratio of lowest postexercise pressure and resting pressure; and whether 5 and 10 minutes after exercise the pressures had reached the pre-exercise level. From their findings they concluded that only three post-exercise measurements within the first $\| 0$ minutes - the first with in two minutes of cessation of exercise - were adequate to uniquely classify the response curve (GAY and ROBERTS 1979).

The question whether pressure measurements during hyperemia give more practical information to the clinician than measurements only at rest has not yet been answered unanimously. In contrast to a majority of authors emphasizing the importance of stress testing (MACKERETH and LENNIHAN 1970; THULESIUS and GJÖRES 1971; CARTER 1972; YAO 1973; CHAMBERLAIN et al 1975; BUTH 1975, 1978; FRONEK et all 1978), a small group of others could not find it of additional value above resting pressure measurement in their studies (DELIUS 1971; HYLKEMA 1975; BRUIJNINCKX 1976). It remains to be seen whether the stress tests employed in these last mentioned studies were adequate.

\subsection{NORMAL PRESSURES AT REST AND DURING HYPEREMIA}

In healthy, non-atherosclerotic, subjects the segmental pressure indices considered as normal, depend on the position and width of the sphygmomanometer cuff employed. The normal values reported in literature range from 1.09 to 1.57 for upper thigh measurements, from 1.18 to 1.32 for measurement just proximal or distal of the knee joint and from 1.07 to 1.20 for the ankle level. Some normal average values for various cuff widths and segmental levels of measurements are summarized in Tabel 5.1. During hyperemia following a walking test no changes in segmental pressure at the upper leg levels could be found compared to resting values (FARIS and JAMIESON 1975; BERNINK 1978). Also at the ankle no substantial changes in pressure occurred in healthy persons after a standard walking test of five minutes (CARTER 1972; SMITH et al 1977; BERNINK 1978). Strenuous exercise, however, caused a short-lasting pressure drop of 25-35 percent of the rest value even in healthy individuals (KING et al 1965; STAHLER and STRANDNESS 1967). Immediately after release of an arterial thigh occlusion in healthy persons, an ankle pressure drop of 17-62 percent of the pre-occlusion value was found with a recovery to normal within 40 seconds (MAHLER et al 1976; HUMMEL et al 1978).

\subsection{CORRELATION WITH THE CLINICAL STAGE OF THE DISEASE}

It is common use to distinguish in atherosclerotic vascular disease the stages of a-symptomatic, intermittent claudication, rest pain and ischemia with necrosis or impending gangrene. Studies correlating thigh pressures with this staging were not found in literature. For ankle pressure indices significant differences between their averages were found for the different clinical stages by YAO (1970, 1973) and THULESIUS (1971). Also the arm-ankle pressure gradient appeared 
TABLE S.I.

Average normal segmental pressure indices ( \pm SD) at the leg, reported in various studies. using different types of sphygmomanometen cuffs.

\begin{tabular}{|c|c|c|c|}
\hline CUFF POSITION & CUFF WIDTH & $\begin{array}{c}\text { NORMAL } \\
\text { PRESSURE } \\
\text { INDEX ( } \pm \text { SD) }\end{array}$ & AUTHORS \\
\hline Upper thigh & $\begin{array}{l}8 \mathrm{~cm} \\
8 \mathrm{~cm} \\
10 \mathrm{~cm} \\
10 \mathrm{~cm} \\
12 \mathrm{~cm} \\
19 \mathrm{~cm}\end{array}$ & $\begin{array}{l}1.57 \pm 0.23 \\
1.51 \pm 0.20 \\
1.53 \pm 0.16 \\
1.37 \pm 0.20 \\
1.34 \pm 0.27 \\
1.09 \pm 0.11\end{array}$ & $\begin{array}{l}\text { HEINTZ et al } 1978 \\
\text { BONE et al } 1976 \\
\text { CUTAJAR et al } 1973 \\
\text { SUMNER and STRANDNESS } 1978 \\
\text { FRONEK et al } 1973 \mathrm{~b} \\
\text { HEINTZ et al } 1978\end{array}$ \\
\hline Above knee & $\begin{array}{l}12 \mathrm{~cm} \\
15 \mathrm{~cm}\end{array}$ & $\begin{array}{l}1.32 \pm 0.23 \\
1.18 \pm 0.10\end{array}$ & $\begin{array}{l}\text { FRONEK et al } 1973 \text { b } \\
\text { BERNINK } 1978\end{array}$ \\
\hline Below knee & $12 \mathrm{~cm}$ & $1.26 \pm 0.24$ & FRONEK et al $1973 \mathrm{~b}$ \\
\hline Ankle & $\begin{array}{l}10 \mathrm{~cm} \\
12 \mathrm{~cm} \\
12 \mathrm{~cm} \\
12 \mathrm{~cm} \\
12 \mathrm{~cm}\end{array}$ & $\begin{array}{l}1.15 \pm 0.08 \\
1.20 \pm 0.09 \\
1.13 \pm 0.09 \\
1.11 \pm 0.10 \\
1.07 \pm 0.15\end{array}$ & $\begin{array}{l}\text { HUMMEL et al } 1978 \\
\text { BERNINK } 1978 \\
\text { SMITH et al } 1977 \\
\text { YAO } 1970,1973 \\
\text { POLLAK et al } 1976\end{array}$ \\
\hline
\end{tabular}

to correlate rather well with the clinical stage of the disease (RAINES et al 1976; Van De WATER 1977; SCHŨTZ 1978). Nevertheless, an individual patient cannot be assigned to one of these clinical groups merely on the basis of the ankle pressure due to the overlap of values for the various stages. The resting ankle pressure index in moderate to severe claudication averages about 0.6 . Significant differences in resting ankle pressure and in pressure fall after a walking test could be found for different degrees of claudication (SMITH et al 1977). Others, however , denied the existence of a simple relation between resting or post-exercise ankle pressures and the severity of the claudication complaints (HYLKEMA 1971; JAUSSERAN et al 1976). Patients suffering from rest pain and ischemic trophic disturbances in general have ankle pressure indices below 0.5 (HYLKEMA 1975; BUTH 1975). The converse is not necessarily true. Regularly patients are seen with ankle pressure indices below 0.5 , yet without rest pain (HYLKEMA 1976). Interesting is the finding of RAINES and co-investigators (1976) that the average ankle pressures of patients with rest pain and ischemic foot lesions with or without diabetes mellitus are different. In diabetics they are significantly higher than in non-diabetics. The authors ascribed this to the high rate of non-compressible arteries in diabetics.

\subsection{RELATION BETWEEN SEGMENTAL PRESSURES AND ANGIOGRAPHIC FINDINGS}

5.5.1. Discrimination between limbs with obstructions and limbs without obstruction at angiography

Discrimination between subjects or limbs with and without angiographic obstructions is very well possible on the basis of ankle pressure criteria 
(FRONEK el al 1973b; HYLKEMA 1976; BRUIJNINCKX 1976; BUTH 1978). In the case of only slight angiographic abnormalities the resting ankle pressure may be normal and the mere employment of this criterion might result in a false (negative) cllassification of the examined subject. In a survey of studies dealing with classification on the basis of resting ankle pressures, false-negatives were found to occur in 2-15 percent of the examined cases (BRULJNINCKX 1976). The existence of an obstructive lesion may be discovered as yet since in those cases the ankle pressure after exercise is abnormal (THULESIUS and GJÖRES 1971; CARTER 1972; BUTH 1978).

5.5.2. Differentiation between single level and multiple level angiographic obstructions

Significant differences were found between the average ankle pressure indices of patients with angiographically prowen total occlusions at one level and those with total occlusions at two levels. But between the average pressure indices of these two occlusion groups and the group of patients with only stenotic lesions no significant differences were found (CARTER 1968, 1969; THULESIUS and GJÖRES 1971; BUTH 1975). Also between the various types of ankle pressure response curves after exercise and the absence or presence of one or more occlusions in the vascular tree a correlation was demonstrated (SUMNER and STRANDNESS 1969; YAO 1973; CHAMBERLAIN et al 1975; GAY and ROBERTS 1979). The considerable overlap of values, however, makes it difficult to assign an individual patient to the group of single level or multilevel obstructive arterial disease on the basis of resting or post-exercise ankle pressures.

\subsubsection{Segmental pressures with well-defined angiographic obstructions}

Isolated total occlusions in the iliac arteries were associated with an average thigh pressure index of 0.6 (normal 1.1) (FARIS and JAMIESON 1975) or 0.51 (normal 0.93) (HURLOW et al 1978) if measured with a broad cuff and of 0.68 (normal 1.53) when determined with a narrow cuff (CUTAJAR et al 1973). This equals a reduction of 45-55 percent compared to the normal resting thigh pressures. In these three publications just mentioned the thigh pressure values for cases with stenoses were found to be in between those of totally occluded and of normal healthy segments. Thigh pressure measurements after a walking test were not significantly different from the values measured at rest both in normals and in patients with aortoiliac occlusions or stenoses (FARIS and JAMIESON 1975; BERNINK 1978). In this relation the observations of FA RIS and JAMIESON (1975) and of EVANS et al (1980) are important. These investigators showed that the inflated blood pressure cuff at the thigh induces hemodynamic changes which nullify the pressure- reducing effect of iliac obstructions. This phenomenon can occur if the stenosis and the cuff are close to each other without open arteries branching off in between to keep the blood flowing through the stenosis during distal cuff occlusion. This allows the pressures proximal and distal to the obstruction to equilibrate. This situation actually never occurs in ankle pressure measurements. A definite increase of pressure fall across aortoiliac obstructions could be found during hyperemia by direct arterial pressure measurements in the common femoral artery. (BLAISDELL and GAUDER 1961; MOORE and HALL 1971; BRENER et al 1974; FARIS and JAMIESON 1975; CASTANEDAZUNIGA et al 1976). 
The resting ankle pressure index in patients with isolated aortoiliac occlusions is diminished down to average values of about 0.5 (NEEDHAM and YAO 1972; CHAMBERLAIN et al 1975; JOHNSON 1975), whereas a walking test induces an average drop of the ankle pressure of $70-90$ percent from the resting level (STRANDNESS and BELL 1964; DEAN and YAO 1976).

Isolated occlusions of the femoropopliteal segment result in a decrease of the resting ankle pressure index to about 0.5 (NEEDHAM and YAO 1973; CHAMBERLAIN et al 1975; JOHNSON 1975). The whole range of values between those of occlusion and the ones of an unaffected vessel is found in stenoses. The ankle pressure fall after exercise caused by a superficial femoral artery occlusion depends on the duration of existence of occlusion. In occlusions of recent onset the pressure still may drop 50-70 percent as compared to the resting level but in occlusions of longer existence, where collaterals could develop, pressure falls of only about 10 percent were found (STRANDNESS and BELL 1964; YAO 1973; LEWIS et al $1972 \mathrm{a}$ ).

According to SIGGAARD-ANDERSEN et al (1972) and BRUIJNINCKX (1976) normal ankle pressures will be found - measured both on the posterior tibial and the dorsalis pedis artery - in case of an isolated occlusion of only one of the lower leg arteries. Such an isolated stop in one of the three below-knee arteries hardly gives any ankle pressure fall during exercise (STRANDNESS and BELL 1964).

Only a few data are available in literature about the influence of disease of the profunda femoris or deep femoral artery on distal segmental pressures. CHAMBERLAIN et al (1975) consistently found a lower resting ankle pressure and a deeper and longer-lasting fall of the pressure after exercise in patients with a concomitant deep femoral artery occlusion in comparison to patients with simillar angiographic abnormalities without such profunda femoris artery obstruction.

The combination of an aortoiliac and a femoropopliteal occlusion causes a reduction of the resting ankle pressure index to 0.15-0.30(CHAMBERLAIN et al 1975; JOHNSON 1975) and it reduces the ankle pressures at exercise tests to unrecordable levels (YAO 1970).

5.5.4. Anatomic diagnosis of arterial obstructions by segmental pressure measurements in the leg

Many investigators have tried to answer the questions whether segmental pressure measurements would allow anatomical localization of arterial obstruction and secondly whether they would permit the differentiation of obstructions of hemodynamic importance in the aortoiliac segment from abnormalities in the leg arteries. This last question is of great importance to the surgeon in the treatment of multilevel arterial disease (see Chapter 1).

Identification of significant aortoiliac obstruction by indirect pressure determination just below the groin appears to be not as simple as might be expected theoretically. Cuffs applied as proximal as possible to the thigh yet lie distal to the femoral artery bifurcation. Therefore, the pressure measurement will be influenced by occlusions at the origin of the superficial and/or profunda fiemoris artery (FARIS and JAMIESON 1975; HYLKEMA 1976; HURLOW et al 1978). By thigh pressure measurement with a broad cuff HEINTZ et al (1978) and HURLOW et al (1978) could not discriminate between stenosis and total occlusion of the aortoiliac pathway. Also the distinction between artoiliac 
occlusion or stenosis on the one hand and occlusions of the superficial femoral artery on the other was impossible with the aid of thigh pressure measurement with a broad cuff (FARIS and JAMIESON 1975). This differentiation could however be made by measuring the thigh pressures with one single narrow cuff (CUTAJAR et al 1973; FRONEK et al 1973 b). Employing a combination of two narrow cuff $s$, one proximally and one distally around the thigh, HEINTZ et al (1978) were able to classify the aortoiliac segments correctly in 79 percent of the cases, also if combined with obstructions in the upper leg arteries.

Anatomical localization of obstructions with the aid of ankle pressure measurement at rest is not possible. It does not permit a differentiation between obstructions in the aortoiliac and the femoropopliteal segment (SUMNER and STRANDNESS 1969; NEEDHAM and YAO 1972; FRONEK et al 1973b). Determination of the ankle pressure indices after exercise did not improve the ability to localize obstructive lesions (BRUIJNINCKX 1976; BERNINK 1978), although STRANDNESS and BELL (1964), YAO (1970) and GAY and ROBERTS (1979) did find a correlation between different ankle pressure response curves and certain anatomical levels of obstruction. Overlapping of response types, however, made their use as a sole parameter unsuitable for the diagnosis in the individual patient.

Combined segmental pressure measurements at various levels allow a reasonably accurate localization of arterial obstruction. In patients with hemodynamically significant obstructions proximal and distal to the groin, both isolated and in combination, BRUIJNINCKX (1976) could predict the abnormality in 88 percent of the cases with the aid of a criterion which was composed of an arm-ankle and arm-thigh pressure gradient "first resting. pressure criterion"). In 73 percent of the cases he was able to distinguish between femoropopliteal and below-the-knee obstructions or their combinations on the basis of the arm-knee and arm-ankle pressure gradients ("second resting pressure criterion"). His method, however, requires a complicated graphic processing which makes it less suitable for routine clinical application. Obstructions in the lower leg arteries correlated reasonably well with the gradients between the suprapatelar and ankle pressure (SIGGAARD - ANDERSEN 1972).

\subsection{CONCLUSIONS}

The heights of the segmental systolic pressures determined in the leg depend on the severity of the obstructive lesions proximal to the cuff, the width of the sphygmomanometer cuffs used and the functional state of the peripheral circulation, i.e. whet her the pressures are measured at rest or during hyperemia. In healthy subjects the segmental leg pressure - measured with appropriate cuffs - will always be at least equal to brachial pressure and usually slightly higher. Ankle pressure levels are correlating with the degree of arterial obstructive disease of the limb, either represented by the clinical severity of the disease or the angiographic appearance of the arteries. The ankle pressure determinations at rest and probably even more reliably after exercise separate subjects without arterial obstructive lesions from vascular patients and thus can serve as a selection or exclusion criterion. However, they do not allow an anatomical diagnosis of obstructive lesions nor a differentiation between aortoillac or femoropopliteal lesions. Thigh pressures are lowered in aortoiliac obstructions and in many cases of (proximal) obstructions of the upper leg arteries. 
Therefore, they have only a limited diagnostic specificity in the identification of aortoiliac obstruction, certainly in multilevel disease. Combined segmental pressure determinations at various levels of the leg allow a reasonable delineation of the distribution of its obstructive lesions. Measurement of systolic thigh pressures under hyperemic conditions appears to have no additional value above determination at rest since no change of these pressures could be found during hyperemia, neither in patients, nor in healthy persons. 


\section{ANALYSIS OF DOPPLER SIGNALS OF THE ARTERIES OF THE LEG}

\subsection{INTRODUCTION}

In peripheral arteries the blood flow velocity changes during the cardiac cycle can be detected by ultrasound Doppler examinations of that vessel. Under normal circumstances these vellocity changes and consequently the character of the Doppler signal, depend on the cardiac function, the compliance of the vascular tree, the site of measurement and the vasomotor tone of the vascular bed supplied by the artery and the rheological properties of the blood (see Chapter 3). Thus normal Doppler signals can be recognized which are characteristic of the arterial site (GOSLING and KING 1974b; GOSLING 1976). The velocity signals of a particular arterial site may be disturbed by such factors as occlusive or other atherosclerotic changes in the vessel wall at the site of measurement and/or its inflow vessels as well as changes in the resistance of the vascular bed supplied by the artery.

The Doppler signals can be submitted to a qualitative or a quantitative analysis. Quantitative analysis requires additional processing of the Doppler sound. This implies for instance a graphic representation of the instantaneous sound frequency spectrum or its derivatives like instantaneous mean or maximum frequency. Elements of the thus recorded signal can then be measured which allows an objective numerical characterization. In the near future direct interfacing of Doppler equipment with computers will probably eliminate the need for a hard copy record of the signal for quantitative analysis. For a qualitative description of the Doppler signal a hard copy record of the signal is also often used. The different signal patterns may then be classified at sight. The most simple qualitative classification however can be achieved by merely listening to the Doppler sound. Some authors distinguished between quantitative and so-called semi-quantitative description of the signals (BERNSTEIN et al 1970; DILLEY and FRONEK 1978). As real quantitative parameters they only considered the ones representing absolute peak or mean velocities of the blood. For this purpose the equipment and its operation have to be standardized and calibrated in animal or in vitro experiments. Analysis which only results in relative values was considered as semi-quantitative by these authors. The latter is usually the case in Doppler examinations where the exact angle between vessel and ultrasound beam is unknown. In this study this differentiation will not be used. A signal analysis is defined as quantitative whenever it gives one or more numerical parameters of the signal or parts of it.

In this chapter a survey is given of the different methods of signal analysis and their diagnostic value is discussed.

\subsection{QUALITATIVE SIGNAL ANALYSIS}

\subsubsection{Acoustic classification}

Arterial Doppler sounds have a pulsatile character which is synchronous with the heart beat. Venous sounds, in contrast, are respiration dependent. The 
Doppler sounds cycle of the main extremity arteries, e.g. the brachial, the femoral, the popliteal or the ankle arteries, consists of two or three high pitched bursts of sound followed by a pause. The first sound directly follows the systolic phase of the heart and the one or two following coincide with the diastolic phase. The second sound burst appears to represent reverse flow. Blood flow in an artery with an inflow obstruction will be damped, resulting in one single systolic Doppler sound, which is also of lower frequency in case of severe proximal obstruction. Flow in an artery which is only filled by collateral circulation around a complete block produces a dull, hardly pulsatile, undulating Doppler sound. Just downstream from an important stenosis, where flow is turbulent, a cyclic high pitched sound can be heard superimposed on a continuous low frequency sound. The systolic sound generated at the level of the stenosis itself is very high pitched and hissing. All these sounds are quite familiar and recognisable to the clinician who regularly uses Doppler equipment and they are quite uniformly described in several publications (e.g. STRANDNESS et al 1967; YAO et al 1968; YAO and BERGAN 1974; DEAN and YAO 1976). One special Doppler sound still needs to be mentioned namely the harsh, pounding, high pitched sound, also described as "water-hammer" sound, which can be heard at the proximal extent of an acute arterial occlusion or a stop in a venous bypass graft (STRANDNESS et al 1967; KEITZER et al 1972).

The quality of the acoustic Doppler signal may be graded in a classifying system. Using a four points scale for the sound quality and comparing the sound scores of the femoral, popliteal and pedal arteries to angiography FELIX et al (1975) were able to predict correctly iliac, superficial femoral and crural artery lesions in 77-100 percent of the cases. THULESIUS and GJÖRES (1971) attributed a score 3 to a high pitched, strong, biphasic sound, corresponding with normal flow in large arteries; a high pitched single sound got score 2 and was considered as denoting a proximal stenosis, except at the ankle where it was assumed to be found also in healthy vessels; a low pitched, dull, sound was given score 1 and considered as belonging to collateral flow distal to an occlusion and score 0 meant no sound audible as is the case with flow velocities below 5 $\mathrm{cm} / \mathrm{sec}$. Using these scores Thulesius came to the conclusion that they were quite unreliable in the evaluation of the arterial circulation (THULESIUS 1971). Different results were obtained by BRUIJNINCKX (1976). Using the same scoring system and monitoring the femoral and pedal arteries, he was able to discriminate between normal and obstructed vessels in 95 percent of the cases. He found an overall accordance between the acoustic Doppler score (called "sonoscore") and the angiographically diagnosed severity and level of the obstructive lesions in 81 percent of his cases. He also found that, contrary to Thulesius" opinion, a score of 2 at the tibial artery indicated pathology. He considered an abnormal sonoscore at the femoral artery as a strong indication of aortoiliac obstruction.

The conclusion is permitted that the simple acoustic Doppler scanning of the peripheral arteries is a good complement to routine physical examination. especially adding to the knowledge of the distribution of the arterial disease in the patient.

\subsubsection{Classifications by pattern recognition}

These classifications are based on a graphic recording of the Doppler signal as a function of time. Both the maximum frequency envelope of a spectrum- 
analyzed Doppler signal and the result of frequency to voltage conversion by a zero-crossing detector give waveform configurations which are quite similar at first glance and which can be classified at sight. In cases of turbulent flow, however, the two processing methods produce different patterns. The spectrographic display shows a uniform increase of both high and low frequency components (spectral broadening) but most of the time a cyclic pattern is still discernable (YAO and BERGAN 1974; ROBERTS et al 1976). The zero crossing meter output becomes completely irregular and spiky in case of severe turbulence whereas in less severe flow profile disturbances the systolic deflections have no round top, but a flattened saw-tooth appearance (YAO 1970; NICOLAIDES et al 1976).

The various classifications which have been published are unanimous in their description of the normal waveform of extremity arteries. It has two or three deflections of which the first - systolic - one has a steep upslope and downslope. The second deflection represents reverse flow and is shown to be negative if directional Doppler equipment is used. Absence of a reverse flow component in the signal under basic resting conditions means in all classifications the first deviation from normal. Further signal degradation is determined by the diminished steepness and height of the remaining systolic peak and the disappearance of a zero level in between (YAO et al 1968; YAO et al 1970; YAO 1970; MATSUO et al 1973; NAYMAN 1974; NIMURA et al 1974).

Figure 6.1.A shows the qualitative Doppler signal classification after Descotes as described in the thesis of MAURIZI (1974). Type 0, the normal pattern, is triphasic and includes a reverse phase. In type 1 the second (reverse) and third (forward) deflection have disappeared. In type 2 the downslope of the remaining deflection is prolonged. In type 3 both the upstroke and the downstroke of the curves are elongated and the zero level between the

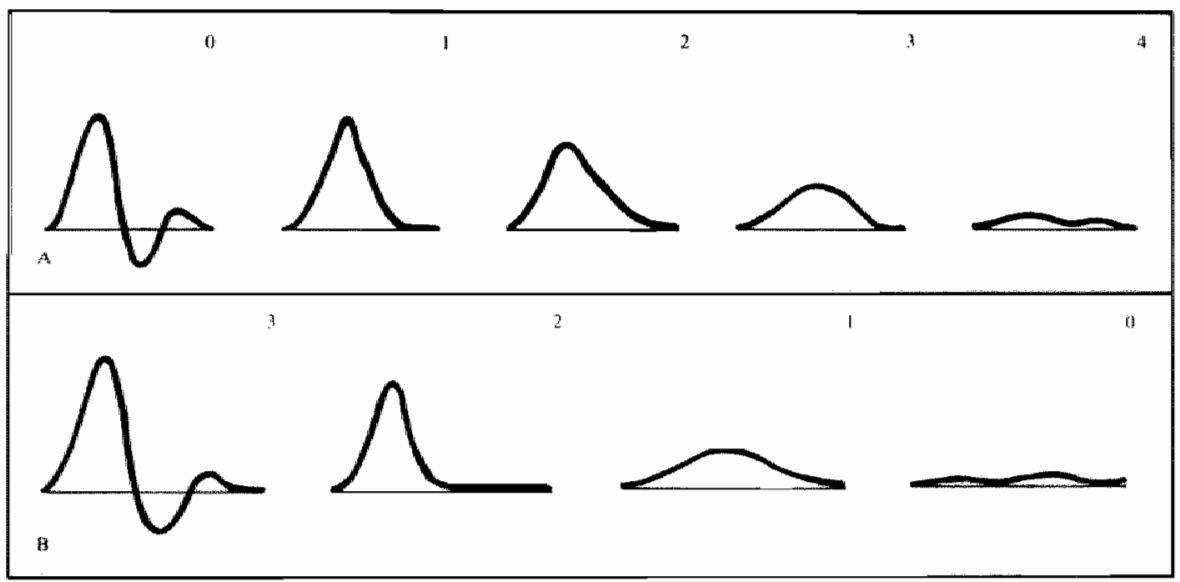

FIGURE 6.1

Classification of Doppler signal patterns.

Normal signals at the left side of the figure with successive degradation of the signats towards the right side of the figure.

A. Classification according to DESCOTES.

B. Classification according to THULESIUS.

See also the text of section 6.2 .2 . 
deflections thas disappeared. Type 4 is a nearly flat registration. Maurizi clamed that these curves contain information about the vascular pathway upstream of the monitoring site only. Curve type 1 would indicate a loss of arterial elasticity rather than a reduction of vascular lumen, whereas type 2,3 and 4 would indicate increasing degrees of proximal obstruction. Comparison of the Doppler curves at common femoral and ankle level (tibial or pedal artery) would allow a topographic diagnosis of arterial obstructions. If the femoral curve is type 0 or 1 and the peripheral classification is 2,3 or 4 this would indicate femoropopliteal lesions. If the signals from both these sites have a classification 2, this indicates iliac or aortic obstruction while a femoral signal type 2 combined with a type 4 curve at the ankle would be indicative of combined iliac and femoropopliteall lesions. This classification agreed well with angiography in over 400 patients. The main advantages of the clinical use of these registrations and their classification were considered to be the confirmation or complementation of results of routine physical and angiographic examinations and the establishment of indications for angiography.

In Figure 6.1.B the Thulesius classification is presented (THULESIUS 1971). The type 3 (normal) waveform configuration is bi- or triphasic and includes a reverse flow deflection early in diastole. Type 2 is a monophasic signal of reduced frequency, whereas type 1 is very low in frequency. Type 0 is a nearly flat trace. No definition of "low frequency" was given by Thulesius. Thulesius found his own classification un reliable in the determination of the severity of arterial occlusive disease of the legs compared to flow studies (isotope and venous occlusion plethysmography) and even more unreliable in comparison to ankle blood pressure measurements (THULESIUS 1971). There are good reasons to believe that the diagnostic results which were obtained by BRUIJ. NINCKX (1976) with a similar - but only acoustic - Thulesius classification (section 6.2.1.) would also have been possible by pattern classification of a graphic record. FARIS and JAMIESON (1975) analyzed the flow velocity patterns of the femoral artery with the purpose of identifying patency, stenosis or occlusion in the aortoiliac pathway. They distinguished between normal, borderline and abnormal patterns both before and after treadmill exercise in a quite equivocal pattern description. The results showed that a correlation existed between the angiographic classification and the type of femoral velocity pattern. However, a remarkably high number of borderline femoral velocity patterns ( 31 percent) and even abnormal signals ( 14 percent) were found in the patients with isolated superficial femoral artery obstructions. No explanation was given for it, but it was suggested that these patients would need further examinations to exclude undiagnosed significant aortolliac disease. In this study the femoral velocity patterns provided a better discrimination between the different aortoiliac lesions than thigh pressure measurements before and after exercise.

It can be concluded that the qualitative classification of Doppler signals by pattern recognition at sight is a worth while addition to routine clinical vascular examination, especially for the delineation of the segmental distribution of obstructions in the arteries of the lower limbs.

\subsection{QUANTITATIVE SIGNAL ANALYSIS}

In order to obtain numerical parameters from the periodic Doppler waveform of which the contours have been described in the previous paragraphs, a variety 
of abvious measurements and calculations can be made (see Figure 6.2.):

- determination of signal amplitudes like the height of maximum positive or negative deflections.

- measurements in the time domain like duration of upslope or downslope of the curve or intervals between curve components.

- integration of the whole curve or parts of it.

- derivatives calculated from these basic measurements like height ratios, time ratios, mean values, peak/mean ratios, acceleration and deceleration.

- Fourier analysis which can only be performed by computer after appropriate presentation of the curve coordinates. This analysis is particularly suited for the periodic phenomena of the cardiovascular system (ATTINGER et al 1966).

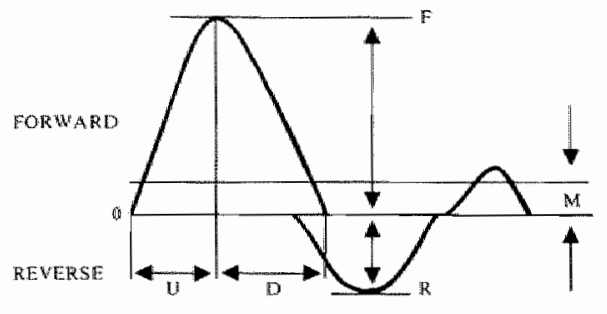

FIGURE 62

Examples of quantitative signal parameters of a schematized bi-directional Doppler registration.

$F=$ maximum height of the forward deflection (frequency or flow velocity), $R$ $=$ maximum height of reverse deflection, $\mathrm{M}=$ mean height over one cycle, $\mathrm{U}=$ duration of the upslope of the systolic deflection, $\mathrm{D}=$ duration of the downslope of the systolic deflection.

In this section a survey will be given of several important Doppler signal parameters. Their clinical and diagnostic meaning will be emphasized rather than the technical problems in obtaining them.

\subsubsection{Signal amplitude parameters}

The instantaneous height of Doppler curves represents the instantaneous peak or mean velocity of the blood flow depending on the type of signal processing used (spectrum analysis or zero-crossing techniques) (sections 4.5.2. and 4.5.3.). Obvious parameters to be derived from these curves are the maximum forward flow velocity, the maximum reverse flow velocity and the mean velocity during one cardiac cycle (see Figure 6.2.).

Especially the surgical and bio-engineering group of Fronek and Bernstein at the University of California has been working with these parameters (BERNSTEIN et al 1970; FRONEK et al 1973a, 1973b; FRONEK et al 1976; DLLLEY and FRONEK 1978). After appropriate calibration procedures (BERNSTEIN et al 1970) they calculated the above mentioned flow velocities - expressed in $\mathrm{cm} / \mathrm{sec}$. - for the femoral, the posterior tibial and dorsalis pedis artery. They found a very good reproducibility of the measurements (FRONEK et all 1976; DILLEY and FRONEK 1978). The parameters of healthy persons were compared to those of patients with isolated significant aortoiliac obstructions or femoropopliteal obstructions or combined lesions. The overall conclusion was that none of their parameters, including the acceleration and deceleration of the systolic velocity, which were calculated as well (see section 6.3.2.), allowed localization of the arterial lesions. For the differentiation between normal subjects and patients with obstructive lesions (all three groups together) the most sensitive para- 
meters appeared to be the deceleration (see section 6.3.2.), the maximum/mean velocity ratio and the maximum forward velocity, all at the common femoral level. The same three parameters together with the maximum reverse flow velocity were helpful in distinguishing multi-level from single-level disease. (DILLEY and FRONEK 1978). On the analogy of what is known about resting volume flow measurements, the resting mean flow velocity was not a reliable index of peripheral arterial occlusive disease in these studies: for this parameter no differences were found between normal subjects and patients. Combination of the Doppler mean velocity measurement in the femoral artery with a postocclusion hyperemia test - in which the relative increase in mean flow velocity and the time for mean velocity to return to half the increase over resting mean value were measured - allowed some, but no full, separation between aortoiliac, femoropopliteal and combined lesions and between normal subjects and patients (FRONEK et al 1976; DILLEY and FRONEK 1978). Others, using a quite similar hyperemia test., found the mean velocity measurement and its changes during hyperemia, measured on the pedal arteries, of little value in the differentiation of arterial acclusions at various levels (MYHRE 1975). The increase in mean velocity which is observed during hyperemia is produced by the disappearance of the reverse flow - if any - and elevation of the diastolic flow velocity level rather than by an increase in the maximum forward flow velocity (MAHLER et al 1976; BLACKSHEAR et al 1979).

The presented normal maximum velocities as found by the group of Fronek and Bernstein were obtained from continous wave Doppler signals processed by a zero-crossing circuitry. They are considerably lower than the normal velocity values in the femoral artery found by MATSUO et al (1973) and NIMURA et al (1974) using continuous wave Doppler and spectrum analysis. This can probably be explained by the fact that a zero-crossed signal approximates to some extent the instantaneous mean blood velocity in the vessel while the spectrum analyzed signal usually provides the instantaneous maximum velocity.

Another parameter which may be derived from Doppler tracings is the ratio maximum reverse/maximum forward amplitude ( $R / F$ quotient). In normal femoral waveforms - where reverse flow is always present - an average $R / F$ quotient of 0.26 ( \pm 0.11 ) was found (RITTENHOUSE and STRANDNESS 1971). In patients with aortic valve incompetence the $R / F$ values of the femoral Doppler signal were on the average much higher due to the regurgitation of blood. Surgical valve replacement restored the femoral $R / F$ quotient to normal. In another, experimental, study the same authors could prove that the $R / F$ quotient of the femoral Doppler curve was linearly related to the resistance of the outflow vessels of the femoral artery. They manipulated the peripheral resistance by induction of post-occlusion hyperemia, hypovolemic shock or int raarterial norepinephrine injections. Increased peripheral resistance caused a reduction of the maximum and mean forward velocity amplitudes of the curves and an increase of peak and mean reverse flow velocities and consequently an increase of both peak and mean $R$. F quotients (RITTENHOUSE et al 1976). In a recent study in which pulsed Doppler equipment was used, the $R / F$ quotients in the common femoral, the superficial femoral and the profound femoral artery near the bifurcation were determined (BLACKSHEAR et al 1979). A significant difference was found between the (lower) $R / F$ quotient in the profound femoral artery and the $R / F$ values in the two other arteries. The authors attributed this to the relatively lower resistance in the arterial bed of the profound femoral artery. Clinical studies relating the $\mathrm{R} / \mathrm{F}$ quotient in peripheral arteries to the 
severity of obstructive lesions could not be found in literature.

The conclusion may be derived that absolute Doppler signal parameters were up till now of only limited value in the evaluation of the vascular patient because they do not allow differentiation due to calibration and basic instrumentation factors. An exception may probably be made for the quotient maximum reverse velocity/maximum forward velocity. This quotient can be related to definite pathological and physiological flow conditions.

\subsubsection{Time-related signal parameters}

An important part of decision making in the process of pattern recognition is based on the steepness of the systolic deflection. This can also be measured objectively and expressed in terms of duration of the upslope period (or pulse rise time) and of the downstroke (or pulse decay time) or in acceleration defined as peak or maximum forward velocity divided by the pulse rise time and deceleration - defined as peak or maximum forward velocity divided by pulse decay time - both expressed in $\mathrm{cm} / \mathrm{sec}^{2}$. It could be observed that isolated proximal obstructions caused a prolongation of both the upstroke and downslope time of the femoral Doppler curve and that isolated obstructions in the superficial femoral artery caused a longer downstroke period of the systolic curve only (NICOLAIDES et al 1976). However, in the same study no significant difference could be found between the measured acceleration and deceleration times of the femoral velocity waveforms in four groups of patients with increasing degress of proximal obstruction. FRONEK et al (1976) and DILLEY and FRONEK (1978), whose amplitude parameters were discussed in the previous section also studied acceleration and deceleration of blood flow velocity in the femoral and ankle arteries. Neither these parameters, nor the ratio acceleration/deceleration could distinguish between patients with isolated aortoiliac or femoropopliteal lesions or combinations of both. The deceleration of the femoral velocity trace allowed a discrimination between the patients and the normals. Because deceleration decreased earlier than acceleration with increasing flow obstruction, the acceleration/deceleration ratio also gave some indication of hemodynamically significant inflow obstruction. The average normal pulse rise times in the femoral, the posterior tibial and dorsalis pedis artery - as recalculated from the tables in a study of DILLEY and FRONEK (1978) - were about 115,110 and $104 \mathrm{~ms}$ respectively. The corresponding pulse decay times were respectively $162, \downarrow 23$ and $121 \mathrm{~ms}$.

The parameters acceleration and deceleration suffer from the same drawbacks as most absolute signal amplitude parameters. They are influenced by such factors as calibration and probe vessed angle, because the value of maximum signal deflection is needed for its calculation. Therefore it seems to be more logical to use the pulse rise time and the pulse decay time as parameters which can hardly be influenced by the position of the Doppler probe or instrument related factors. (HUMPHRIES et al 1980).

It can be concluded that time-interval parameters of the systolic part of the Doppler signal of certain arteries may contain information about the intlow and outflow condition of that artery, but no quantitative data are avallable yet.

\subsubsection{Pulsatility index}

In order to compare the blood flow velocity waveforms obtained at different 
sites in the same patient or from different patients it would be necessary to eliminate the calibration factor which influences the amplitude of each Doppler curve. To this purpose some form of normalization is required. It could be shown - by computing the percentage energy in each Fourier harmonic of the waveform - that the basic shape of the maximum frequency envelope of a spectrum analyzed Doppler signal was uninfluenced by the angle between the Doppler probe and the direction of flow or by the flow profile (WOODCOCK et al $1972 \mathrm{~b}$ ). Fourier analysis is a simple and elegant way of mathematically describing the complex periodic waveforms originating from the oscillatory system of the peripheral arterial tree. This holds for pressure/time, volume flow/time (STRANDNESS and SUMNER 1975, p. 75-78) and flow velocity/time waveforms (WOODCOCK et al 1971). The instantaneous velocity/time waveform may be regarded as a steady forward flow velocity on which is superimposed a stationary oscillatory flow consisting of a number of harmonic components. The first component has the same period as the cardiac cycle, the second one has half of this period, the third harmonic has one-third of it and so on (section 3.2.). Using this Fourier analysis a normalization of the flow velocity/time waveform could be introduced in which the whole waveform was characterized by one single dimensionless figure, the pulsatility index (WOODCOCK et al 1971, 1972a; GOSLING et al 1972). This index is independent of calibration influences like probe-vessel distance or angle between the ultrasound beam and the flow direction.

The pulsatility index (PI) is defined as the sum of the maximum oscillatory energy of the Fourier harmonics of the waveform divided by the mean energy (mean flow velocity) over a cycle.

To obtain the Fourier harmonics the waveform needs to be digitized in $x$ and $y$ coordinates to allow computerized analysis.

The described (Fourier) PI was used in a number of studies in which mostly the PI proximal to a vascular segment was compared to the distal PI, thus allowing the calculation of a "damping factor". This segmental parameter is described separately in Chapter 7 . The normal values of the Fourter PI obtained from young, healthy men and women for different vessel sites are: abdominal aorta 2-6; common femoral artery 4-13; popliteal artery 6-18; posterior tibial artery 8-26 (WOODCOCK et al 1971). No essential differences were found between the values for male and female subjects. The PI value clearly increased progressively from the aorta to the ankle. In a study of 45 angiographed patients with intermittent claudication and 30 asymptomatic normal subjects (HARR IS et al 1974), the femoral PI values differed significantly between patients with various degrees of aortoiliac obstruction. In case of an unaffected aortoiliac segment the femoral PI averaged 7. $1 \pm 1.8$ and gradually decreased to $1.6 \pm 0.9$ in case of a complete occlusion. With only few exceptions, the PI in the common femoral artery was below the lower limit of normal in all patients who had angiographic evidence of stenosis in the aortoiliac segment. In cases of isolated femoropopliteal obstruction the average popliteal PI's did not differ significantly for the three classes of obstruction which were distinguished in this study, although the decrease in popliteal PI paralleled the severity of the femoropopliteal lesions. There was however a significant difference in popliteal PI between "normal" and affected femoropopliteal segments. The PI of the posterior tibial artery did not correlate with the angiographic condition of the lower leg vessels but allowed a differentiation between normals and patients. Johnston and co-investigators performed a comparable study in which similar 
figures were found. Their normal common femoral artery PI averaged $9.9 \pm 5.7$ and the pedal PI had a normal average of $17.1 \pm 7.8$. In their study the femoral PI correlated well with the severity of aortoiliac arteriographic grading (JOHNSTON et al $1977 \mathrm{a}$ ).

The complexity of the Fourier PI determination - necessitating the use of a computer - would probably have precluded its further clinical application. It could however be shown that a more easily obtained pulsatility index - called the "peak-to-peak" pulsatility index - bears a close relation to the Fourier PI (GOSLING and KING 1974b; JOHNSTON et al 1977a). This peak-to-peak PI (Figure 6.3.) is defined as the ratio of the peak-to-peak distance of the curve
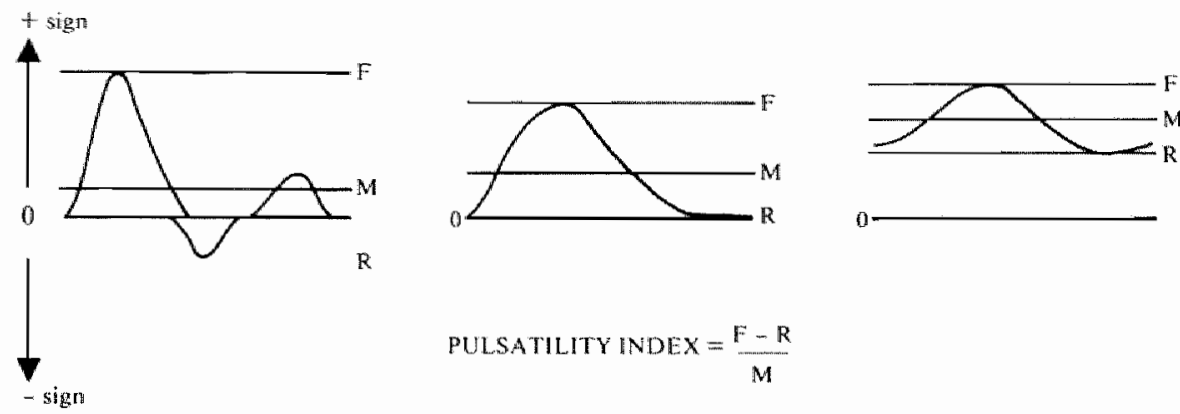

PULSATILITY INDEX $=\frac{F-\mathbb{R}}{M}$

\section{FIGURE 6.3}

Definition of the Pulsatility Index

$\mathrm{F}=$ maximum forward deflection of the curve, $\mathrm{M}=$ mean level of the curve over one cycle calculated by subtracting the area under the reverse curve, if any, from the total area under the forward curve(s) and dividing it by the duration of the cardiac cycle, $R=$ maximum reverse deflection, if any, with a negative sign (left figure), or lowest positive deflection in the absence of a reverse component (right and middle figure).

(including reverse deflections) over the mean height of the curve per cycle. The peak-to-peak distance can simply be measured with a ruler. The mean height is calculated by measuring the total area under the curve (e.g. with a planimetric device), making allowance for its + or - sign and dividing it by the cycle length. Alternatively the height at equally spaced intervals throughout the cycle may be averaged (JOHNSTON et al 1977b).

A diagrammatic representation of the approximate rellationship between the simplified peak-to-peak PI and the Fourier PI, composed from the data contained in two studies in which both indices were calculated in pairs from a great number of Doppler registrations (GOSLING and KING $1974 b_{;}$JOHNSTON et al 1977a) is given in Figure 6.4. Although it is not completely clear why the two pulsalitity indices should be related to each other, it seems justifiable to regard the more simple peak-to-peak PI as an equivalent to Fourier PI.

In the following all presented PI values will be those of "peak-to-peak" calculations. In an animal study the application of progressively increasing artificial iliac artery stenoses, applied $10 \mathrm{~cm}$ proximal to the common femoral artery, caused a progressive diminution of the PI of the femoral artery. A highly significant inverse linear relationship was found between the PI and the percentage stenosis. Changes in PI occured at subcritical levels of arterial obstruction before detectable decrements in pressure or mean volumetric flow 


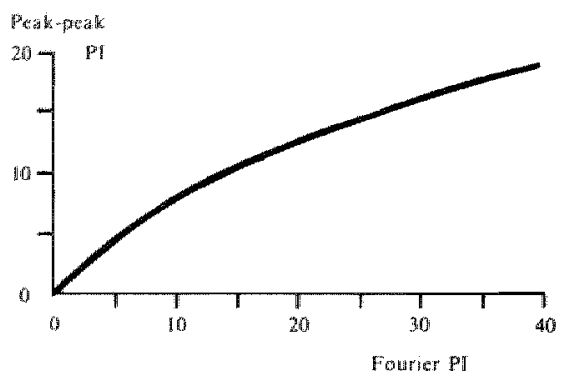

FIGURE 6.4

Correlation between the Pulsatility Index found by Fourier analysis (Fourier PI) and the peak-to-peak PI from the same Doppler curwes.

For definitions and references see section 6.3 .3$.

(BONE and AMMONS 1978). The range of normal values for the peak-to-peak PI, as presented by GOSLING and KING (1974b), is: abdominal aorta 2-6; common femoral artery $5-10$; popliteal artery $6-12$; ankle arteries $7-15$. It is obvious that these normal values differ slightly from the normal Fourier PI's as determined by the same authors (GOSLING et al 1971). A good correlation between this PI of the Doppler signal of the common femoral artery and an angiographic grading of obstructions in the aortoiliac segment - subdivided in normal, minimal irregularities, less than 50 percent diameter stenosis, more than 50 percent diameter stenosis and occlusion - was claimed in a series of publications by one group of investigators (JOHNSTON and TARASCHUK 1976; JOHNSTON et al 1977a, 1977b). The overlap of the value for the neighbouring angiographic classed did not alllow a full discrimination between these classes on a femoral PI basis, but aortoiliac segments with stenosis over 50 percent could be separated from normal segments. Comparable findings for the PI in the diagnosis and assessment of aortoiliac disease were reported by WARD and MARTIN (1980). The use of the PI at ankle level for the discrimination between increasing degrees of angiographic abnormalities, graded by a single figure for the whole vascular tree from the aorta down to the ankle level showed that separation was only possible between a normal and a severely affected vascular system (JOHNSTON et al 1977b).

A further deviation from the original concept of pulsatility index as developed by Gosling and Woodcock is found in those studies which use the waveform of a zero-crossed Doppler signal for the calculation of the peak-topeak PI. Then, an important prerequisite for the calculation of the PI is probably not fulfilled, namely, that the waveform pattern does not change over a wide range of Doppler probe/vessel angles as it is virtually the case for the maximum frequency envelope of a spectrum-analyzed signal. (WOODCOCK et all 1972b; JOHNSTON et al 1977b). In one such study a "waveform index" - of which the definition was similar to that of the peak-to-peak PI - was calculated for the femoral (zero crossed) Doppler signal. Different angiographic groups of inflow obstruction to the femoral artery could not be fully separated on the basis of this parameter, although an apparent correlation existed between this femoral waveform index and the severity of the obstruction (NICOLAIDES et al 1976). In another preliminary study on the femoral PI, as calculated from a zero-crossed Doppler signal, it was concluded that it allowed a good judgement of the iliac arteries (ALTSTAEDT et al 1979).

However, recently the value of the common femoral PI in the assessment of aortoiliac obstruction has repeatedly been relativized. WARD and MARTIN (1980) who found a trend towards progressive diminution of the femoral PI with increasing severity of iliac disease, also found a significant difference in the 
mean value of this PI between patients with an isolated superficial femonal artery occlusion and normal subjects. On the other hand they could not demonstrate a significant difference in mean femoral PI value between patients with isolated proximal disease and those with an equal grade of aortoiliac disease plus a superficial femoral artery block. Their conclusion was that the result of the common femoral PI determination might be influenced by distal as well as proximal disease, although when both coexist the effect of the latter will overshadow that of the former. In another recent study a good correlation of the femoral PI with the degree of iliac stenosis could only be found if the vessels distal to the common femoral artery were patent. If the superficial femoral artery was occluded this PI did not correlate with the presence or extent of illac disease (BAIRD et al 1980).

It seems justified to draw the conclusion that the pulsatility index of the Doppler signal is of clinical relevance. At the femoral artery level its determination may be helpful in the delineation of the severity of aortoliac obstructions but its value may be considerably reduced in cases of multilevel disease.

\subsubsection{New developments in Doppler signal analysis}

Very recent reports mention a new method of Doppler signal analysis, called Transfer Function analysis or Laplace Transfer analysis. This purely computerized signal processing technique expresses the waveform shape mathematically by a curve-fitting technique and provides three coefficients of the third order Laplace transform which can be related respectively to distal impedance. proximal lumen diameter and vessel wall elasticity (BROWN et al 1978; SKIDMORE et al 1979; BAIRD et al 1980; RENEMAN 1981). It was claimed that its application on the Doppler signal of the common femoral artery resulted in a more sensitive technique than the PI in the detection of incomplete iliac artery stenosis and that it would be less influenced by concomitant obstruction distal to the groin than the femoral PI (SKIDMORE et al 1979; BAIRD et al 1980). Although the first clinical results look promising, further clinical evaluation of this signal analysis technique is necessary to rate the preliminary findings at their true value. 


\section{SEGMENTAL DOPPLER PARAMETERS OF THE LOWER EXTREMITIES}

\subsection{INTRODUCTION}

A close phasic relation exists between the pressure pulse wave and the flow velocity waveform at a particular site as shown in Chapter 3 . The pressure pulse propagation time along an arterial segment of some length depends largely on the compliance of the vessel wall (MCDONALD 1974, p. 389-419; GOSLING and KING 1974b; GOSLING 1976). Changes in this compliance cause a change in the propagation time of both the pressure wave and the related velocity wave. Thus, the delay between arrival of the velocity wave at the entrance of an arterial segment and at the end of it - also called the transit time of that wave - is an indication of the compliance of that segment. If the pulse wave had to travel along a tortuous and long collateral pathway around a complete arterial block, the transit time over the occluded segment is prolonged. Doppler equipment is very useful in monitoring the arrival of a (velocity) pulse wave at a given site, because it registers changes in blood velocity during the cardiac cycle accurately. If two Doppler probes are operated simultaneously at the entrance and exit of vascular segment, the transit time of a pulse wave along that segment can be recorded beat-by-beat. An alternative method of transit time determination - for which only one Doppler probe is needed - is based on the measurement of the time delay between some characteristic point of an ECG signal and the moment of arrival of a corresponding velocity pulse wave in a peripheral artery. For the determination of the transit time along a specific vascular pathway these time delay values measured distally and proximally in that segment are subtracted from each other (HARPER et al 1974; BRUINS SLOT 1981).

Obstruction to flow in an arterial pathway produces a damping of the Doppler signal wave shape (section 3.5.2.3). To determine this damping the waveform at the proximal and the distal end of the vascular segment should be compared. This is only possible objectively if both waveforms can be described in a numerical way and if the waveform description will not be influenced by differences in recording circumstances at both sites. The pulsatility index (PI) discussed in the previous chapter as a numerical parameter virtually independent of the angle between the ultrasound beam and the direction of blood flow was therefore used to calculate a damping factor for arterial segments (WOODCOCK et al 1972a; GOSLING et al 1972; GOSLING and KING 1974b). This damping factor is defined by the ratio of the PI at the proximal site of the segment to the PI at the distal site. The PI distal to an arterial segment is largely determined by the degree of stenosis in that segment. However, also the proximal PI is influenced by a stenosis in the segment due to reflections from the stenosis. Both the proximal and distal PI are further determined by local vessel wall elasticity, whereas the distal PI value depends largely on the peripheral vascular resistance (RENEMAN 1981; see also section 6.3.3.). Consequently the damping factor, as defined above, can not be considered merely a parameter of obstruction in an arterial segment. Yet, in many studies the damping factor was found to give valuable information on the degree of obstruction in an arterial segment. 
Both the transit time of a velocity waveform and the damping factor of an arterial segment will be called "segmental parameters". They should be distinguished from "signal parameters" as described in Chapter 6. In this chapter some aspects of transit time measurement and damping factor determination as well as their value in the diagnosis of arterial obstructive disease will be dealt with. The reason to discuss both parameters together in one chapter is induced by the fact that from the beginning both parameters have been used in combination to describe the dynamic capability of arterial segments (FITZGERALD et al 1971; GOSLING et al 1972; WOODCOCK et all 1972a). In later publications this approach was continued.

\subsection{TRANSIT TIME MEASUREMENT}

To measure the transit time of a velocity waveform between two sites in a vascular pathway the Doppler signals from both sites are recorded and displayed simultaneously. By measuring the distance along the time axis between two identical points at the two curves the delay between the velocity waves is determined. The measurement can be performed manually with a ruler or on a graphic tablet connected to a dedicated computer (GOSLING 1976) or with a special purpose transit time computer (KING et al 1972). Quite unanimously the foot-to-foot distance of the systolic upslopes is determined (MCDONALD 1974, p. 392, 393; RENEMAN 1981), the reason for this being the fact that at the start of systole all harmonics of the curve are in phase whereas measurement of the time lag between systolic peaks might be influenced by reflections from the periphery (GOSLING and KING 1974b; GOSLING 1976; RENEMAN 1981). It could be shown that the transit time of velocity waveforms does not only depend on the age of a subject but also on the mean arterial blood pressure in the vascular segment under investigation (KING et al 1972; GOSLING 1976). For this reason frequently the observed transit time is "corrected" or "normalized" for age and blood pressure by dividing it by the transit time which is considered to be normal for a similar but patent pathway in a comparable age group at the mean blood pressure of the tested individual. (GOSLING and KING 1974a, 1974b; GOSLING 1976). Diagrams from which these normal transit times values might be extrapolated are given in the three last mentioned publications. GOSLING (1976) emphasized that the influence of respiration on the mean blood pressure would make it necessary to average the transit times over at least 20 successive heart beats, i.e. about four respiration cycles.

The range of normal foot-to-foot velocity pulse transit times for the vascular segments of the leg were found to be: for the aortofemoral segment $20-35 \mathrm{~ms}$ in young adults and 15-25 ms in normal subjects of higher average age. For the femoropopliteal pathway a transit time of $30-45 \mathrm{~ms}$ was found and for the popliteal-to-posterior tibial artery segment a transit time of $35-55 \mathrm{~ms}$ (FITZGERALD et al 1971; GOSLING et al 1971; GOSLING and KING 1974a, 1974b).

The data presented in literature on the transit times for which an ECG signal was used as reference are rather at variance whereas their comparison is hindered by a non-uniform selection of reference points at the ECG and the Doppler curves (RITTENHOUSE and STRANDNESS 1971; NIMURA et all 1974; HARPER et al 1974). One interesting application of the measurement of the time delay between the ECG and a femoral Doppler signal was published by Waters and co-investigators. They described a parameter which was called the Proximal 
Damping Quotient (PDQ). This was defined as the ratio of the time delay between the R wave of the ECG and the systolic peak of the Doppler curve and between the $R$ wave and a point halfway the systolic upslope. With this femoral PDQ a good separation was possible between pattents with proximalobstruction and patients without. It could be concluded that a PDQ below 1.4 nearly excluded significant obstruction in the aortoillac segment, which would make this PDQ a significant parameter of aortoiliac obstructive disease (WATERS et al 1977).

\subsection{DAMPING FACTOR}

The concept of the damping factor was worked out simultaneously with that of the pulsatility index by the vascular investigation group at Guy's Hospital in London (FITZGERALD et al 1971; GOSLING et al 1972; WOODCOCK et al 1972a). In most of their studies the determination of the damping factor was combined with measurements of transit times to obtain functional information about specific vascular segments (section 7.4.). On the analogy of the normalization of the transit time (section 7.2.) normalization of the damping factor is also possible. For this purpose the observed damping factor is divided by the average damping factor for the same vascular segment obtained from measurements of a group of normal persons.

The average normal damping factors for persons between 20 and 50 years of age as reported by the group of Gosling were: in the femoropopliteal segment $0.79 \pm 0.30$ and in the popliteal-to-posterior tibial segment $0.94 \pm 0.35$. (GOSLING and KING 1974a; GOSLING 1976). In normal resting persons the damping factor in successive limb segments is always less than 1.0 (FITZGERALD et al 1971; GOSLING et al 1971). Published studies about the damping factor nearly exclusively deal with the femoropopliteal or popliteal-to-ankle segments. No normal values of the damping factor could be found for the aortoiliac pathway. An indication of the difficulties encountered when attempting to calculate the damping factor for the iliac vessels is given by GOSLING (1976). He noticed short-term variations in waveform shape of Doppler signals from the abdominal aorta which were not recognized in simultaneously recorded common femoral waveforms of the same subject. Gosling suggested that these variations might be due to variable flow rates in the hypogastric arteries.

In a few studies the damping factor by its own was correlated with angiography. In one study it was found that the damping factor of the lemoralto-posterior tibial segment could reliably predict the angiographic condition of the arteries in the leg with regard to stenosis or total occlusion of the superficial femoral artery or the presence of multiple lesions in the leg. However, separation of the stenotic from the normal superficial femoral arteries was far from perfect (ANGELIDES et al 1979). In studies on the femoropopliteal pathway the segments with a partial obstruction were not consistently distinguished from patent segments with the use of the damping factor (JOHNSTON et al $1977 \mathrm{~b}$; BR UINS SLOT 1981). It could be established that both the damping factor in the femoropopliteal pathway and in the whole leg (from groin to ankle) were not influenced by concomitant aortoiliac disease in the limb (ANGELIDES et al 1979; BRUINS SLOT 1981).

An interesting new development is the calculation of a damping factor across the common femoral artery bifurcation. With the use of pullsed Doppler 
equipment it is possible to obtain selectively curves from the common femoral artery proximal to the bifurcation and of the superficial femoral and profound femoral artery behind this bifurcation (see e.g. BRUIN SLOT 1981). Comparison of the pulsatility indices of these curves can give the damping factor along the origin of the profunda femoris artery. The values of this damping factor could be compared to operative findings and definite limits were established beyond which the origin of the profound femoral artery had a certain significant stenosis and below which it could be considered normal (BAIRD et al 1979). In view of the importance of the deep femoral artery in case of a superficial femoral artery occlusion and the failure to demonstrate stenosis at its origin in routine angiography, this may be considered to be an important development.

\subsection{COMBINED USE OF TRANSIT TIME AND DAMPING FACTOR}

Gosling and co-workers plotted transit times and damping factors of upper and lower leg segments against each other in scatterdiagrams. Each of the obtained points in that diagram was labelled according to the angiographic grading of the corres ponding vascular segment. Four angiographic classes were distinguished: (I) complete block with established collaterals; (II) severe stenosis; (III) "mismatch" of the wessel which meant narrowing over most of the pathway or a diffuse dilatation of the vessel and (IV) patency without important lesions. A clear, four quadrants, division of the diagram field became apparent, each containing the majority of (damping factor/transit time) coordinates for one angiographic class. This applied to both femoropopliteal and the popliteal-toposterior tibial segment. Using this quadrant-based classification system on a material of $85 \mathrm{leg}$ segments, 72 percent of the segments could be classified correctly (considering the angiogram as the standard), 24 percent was classified incorrectly and 4 percent of the classifications were considered as borderline (GOSLING and KING 1974a). This classification could be improved considerably by employing the "normalized" damping factor and transit time (see section 7.2 and 7.3). Agreement between this ultrasound classification and angiography could in this way be improved to about 90 percent even in the presence of concomitant proximal disease (GOSLING and KING 1974a; GOSLING 1976). Using the same techniques and employing the same diagnostic levels HUMPHRIES and co-investigators (1980) could not reproduce these results in a large study of over 300 femoropopliteal segments of patients and normal subjects. Normal and totally occluded segments could be allocated to two separate quadrants. This work did neither confirm, however, the finding of separate quadrants for "mismatch" and stenosis nor could normalization of the parameters improve the separation between the groups. GOSLING and KING (1974b) also devised another classification which described the total vessel condition and which could, according to them also be extended to the aortofemoral segment. This classification allegedly allowed judgement of the luminal reduction and the degree of wall stiffness due to atheromatous involvement by comparing the observed segmental transit time with the normal transit time and by the determination of the damping factor. How vessel wall stiffness could be read from an arteriogram was not mentioned.

Another application of the combined measurement of transit time and damping factor as indicated by Gosling's group is in grading the functional capacity of collaterals around a total occlusion. This was based on the rather subjective impression that the femoropopliteal transit time had a relation to the 
length of collateral vessels around a superficial femoral artery block and that the damping factor was related to the calibre of these collaterals as seen on an angiogram (GOSLING 1976). Four classes of hemodynamic effectiveness of collaterals were distinguished after the four possible combinations of short or long and wide or narrow collaterals. It was possible to establish threshold levels for absolute and normalized transit time and damping factors in the femoropopliteal pathway allowing a discrimination between these four classes of collateral development around a total stop (FITZGERALD et al 1971; GOSLING et al 1972; GOSLING and KING 1974b; GOSLING 1976). The worse this collateral classification for a block in the superficial femoral artery was, the more the walking distance decreased and the greater the systolic pressure drop was across the segment (GOSLING 1976). In 25 patients with an occlusion of the superficial femoral artery the systolic arm-ankle pressure gradient and the maximum hyperemic calf flow (determined by strain-gauge plethysmography) were correlated with the femoropopliteal transit time and damping factor. The transit time showed a good linear correlation with the pressure and flow parameters. So did the damping factor, though to a lesser degree (WOODCOCK et al 1972a). These findings support the concept of grading collaterals around a total arterial block by measurement of damping factor and transit time. 


\section{THE EVALUATION OF THE AORTOILIAC PATHWAY BY SEGMENTAL SYSTOLIC PRESSURE MEASUREMENT, DOPPLER SIGNAL ANALYSIS AND SEGMENTAL DOPP- LER PARAMETER DETERMINATION}

In previous chapters a general review on methods and clinical relevance of segmental systolic pressure readings, analysis of the Doppler signal and determination of segmental Doppler parameters was given. In this chapter the single as well as the combined use of these tests in the assessment of obstructions in the aortoiliac segment only - as compared to angiography - will be reviewed.

The diagnostic value of a test parameter is determined by its ability to detect or exclude pathologic conditions. A usual way to express the value of any test is by calculation of its diagnostic accuracy, specificity and sensitivity, using a so called decision matrix (MCNEIL et al 1975). The sensitivity expresses the ability of the test to discover abnormalities or disease and is diminished in case of many false-negative test results. The specificity of a test is a measure of its ability to exclude the normals and it is reduced if many false-positives are found by it. Accuracy is the total percentage correct test results. In most older, otherwise valuable, publications test results are not expressed in terms of accuracy, specificity and sensitivity. However, sometimes the presentation of the data allows the calculation of these modes as yet. All publications used as sources for the chapters about segmental pressure measurements (Chapter 5), Doppler signal analysis (Chapter 6) and segmental Doppler parameters (Chapter 7) and other publications about the combined use of such tests were scrutinized for the possibility to calculate the diagnostic ability of the presented test, expressed in the described modes. The results are discussed in the following sections and presented in Tabel 8.1. The figures should be looked at very carefully because they are taken from patients series of different composition while the parameter thresholds were not always the same. Care was taken to select only those studies for this retrospective calculation in which at least aortoiliac, femoropopliteal and combined obstructions were evaluated by the test. Normal controls were excluded from the calculations but angiographically normal segments of patients were included. Only the accuracy, specificity and sensitivity in the diagnosis of aortoiliac obstructive disease were considered.

\subsection{SEGMENTAL PRESSURE MEASUREMENT}

Systolic ankle blood pressure measurement is very useful in the estimation of the functional severity of the obstructive arterial disease of the leg but does not give anatomical information. Therefore, it can not be considered a suitable parameter in the detection and quantification of aortoiliac obstructive lesions (Chapter 5).

Thigh pressure measurements have a controversial place in the evaluation of the aortoillac segment. Occlusions of the origin of the superficial femoral artery lower the thigh pressure index irrespective of the severity of the aortoiliac disease. This reduces the specificity of this test. Also the cuff width influences the discrimination between aortoiliac stenosis or occlusion and between isolated aortoiliac obstruction and combined aortoiliac/femoropopliteal obstruction (Chapter 5). Even the simple conclusion that a normal thigh pressure index excluded significant aortoiliac obstruction (CUTAJAR et al 1973) was 
TABLE \&.1.

Accuracy, specificily and senstivity in diagnosing significant aortoiliac obstruction. Retrospectively calculated from data in the mentioned publications.

\begin{tabular}{|c|c|c|c|c|}
\hline AUTHORS & PARAMETERS & ACCURACY & SPECIFICITY & SENSITIVITY \\
\hline HYLKEMA 1975 & Thigh pressure & 80 & 73 & 90 \\
\hline $\begin{array}{l}\text { FARIS and } \\
\text { JAMIESON } 1975\end{array}$ & Thigh pressure & 61 & 46 & 93 \\
\hline HEINTZ et al 1978 & $\begin{array}{l}\text { Thigh pressure } \\
\text { (cuff } 19 \mathrm{~cm} \text { ) } \\
\text { Thigh pressure } \\
\text { (cuff } 8 \mathrm{~cm} \text { ) }\end{array}$ & 83 & 74 & 95 \\
\hline $\begin{array}{l}\text { BRUUJNINCKX } \\
1976\end{array}$ & $\begin{array}{l}\text { Combined pressure } \\
\text { thigh/ankle }\end{array}$ & 94 & 97 & 82 \\
\hline HEINTZ et al 1978 & $\begin{array}{l}\text { Combined pressure } \\
\text { thigh high/low }\end{array}$ & 96 & 96 & 95 \\
\hline $\begin{array}{l}\text { BRUIJNINCKX } \\
1976\end{array}$ & $\begin{array}{l}\text { Femoral Doppler } \\
\text { "sonoscore" }\end{array}$ & 84 & 82 & 92 \\
\hline WATERS el al 1977 & $\begin{array}{l}\text { Transit time ECG - } \\
\text { femoral Doppler }\end{array}$ & 82 & 72 & 93 \\
\hline YAO 1970 & $\begin{array}{l}\text { Ankle pressure/ } \\
\text { velocity pattern }\end{array}$ & 84 & 39 & 99 \\
\hline
\end{tabular}

denied by others (FRONEK et al 1978). These facts are reflected in the diagnostic accuracy, specificity and sensitivity percentages for single-level thigh pressure determinations as given in Table 8.1. The lower specificity of the thigh pressure measurement in the series of Faris - due to an extreme high number of abnormal thigh pressures in the angiographic group of "isolated superficial femoral artery occlusion" - very likely results from an angiographic underestimation of lesions in the aortoiliac pathway. This is supported by the finding that the Doppler signals of the common femoral artery were abnormal or borderline in nearly half of these cases (FARIS and JAMIESON 1975).

By the combination of two segmental pressure measurements, one proximal at the thigh and one at a more distal position, femoropopliteal obstructions are better recognized which consequently improves the accuracy and specificity of this segmental measurement in the detection of aortoiliac disease (HEINTZ et al 1978; Table 8.1.). BRUIJNINCKX (1976) developed a quite complicated method to identify significant obstruction in the various vascular segments of the leg by comparison of the ankle - arm and the thigh - arm pressure difference. As can be seen from Table 8.1., this method allowed a very reliable diagnosis of aortoiliac disease compared to angiography.

\subsection{DOPPLER SIGNAL ANALYSIS}

A simple qualitative analysis of Doppler sounds from the femoral artery has 
been used in a few studies for the characterization of the aortoiliac pathway and for comparison with the angiographic diagnosis. (section 6.2.1. and 6.2.2.). The study of BRUIJNINCKX (1976) permitted a calculation of the diagnostic value and is presented in Table 8.1. He used a Thulesius classification (section 6.2.1.) of the femoral Doppler sound and considered significant aortoiliac obstructions present in all the cases where the score was less than 3 . The value of this parameter was very reasonable and comparable to that of pressure measurements.

Of the quantitative Doppler signal parameters the amplitude derivatives and the time-related ones (section 6.3.1. and 6.3.2.) have not yet been found of great relevance in the assessment of the severity of peripheral vascular disease. No factual data could be found about their single use in grading aortoiliac obstruction which allowed the judgment of their diagnostic value.

The pulsatility index (PI) of the Doppler signal of the common femoral artery is a parameter with a good relation to the degree of aortoiliac obstruction as shown in section 6.3 .3 . However, in none of the publications referred to in that section the authors have given exact figures as to correlation to angiography and the thresholds of the PI indicating significant stenosis. Thus, the exercise of calculating the diagnostic accuracy, specificity and sensitivity of the PI could not be undertaken.

\subsection{SEGMENTAL DOPPLER PARAMETERS}

The data about damping factor and foot-to-foot transit time measurements (sections 7.2 and 7.3 ) are nearly exclusively available for the segments of the legs and not for the aortoiliac pathway. It has been stated that the combined measurement of transit time of the velocity waveform and damping factor across the aortoiliac pathway was equally valuable as that along the femoropopliteal segment (GOSLING and KING 1974b, 1978). However, figures comparing the results of these parameters for the aortoiliac pathway with anglography are not available.

Waveform transit time values derived from the time lag between the ECG and the peripheral Doppler signal have been correlated with aortoiliac vessel conditions. One of such parameters which appeared to permit a quite correct diagnosis of aortoiliac obstructive disease was the so called Proximal Damping Quotient (WATERS et al 1977, section 7.2.). Its diagnostic accuracy, specificity and sensitivity is given in Table 8.1.

\subsection{COMBINED USE OF PRESSURE AND DOPPLER SIGNAL OR SEGMEN- TAL DOPPLER PARAMETERS}

In early publications about the use of Doppler equipment in the hemodynamic evaluation of peripheral arterial disease, measurement of systolic segmental pressure combined with analysis of the quality of the signal was already used to identify aortoiliac obstruction. YAO (1970) distinguished four types of posttreadmill-exercise pressure/flow velocity patterns at the ankle level. The pressure response curves were classified in four types and were determined by the percentage pressure drop and the recovery time of the pressure after exercise (section 5.2.). This information was complemented with that of Doppler signal characteristics at the ankle where either an increase of velocity could be noticed or a decrease with a gradual loss of pulsatile characteristics. The less disturbed 
pressure/velocity patterns (type l) were nearly all found in cases of simple single superficial femoral artery occlusions, whereas iliac stenosis and obstructions were characterized by moderately and severely disturbed, type III and III, pressure/velocity patterns. The majority of patients with combined aortoiliac and femoropopliteal lesions had type II, III or IV pressure/flow patterns at the ankle. Using a type II, III or IV post-exercise pressure/velocity pattern as criterion for aortoiliac obstruction, the diagnostic value of this criterion compared to angiography, was calculated from the data presented in Yao"s publication (see Table 8.1.). The specificity of this method is too low to make it a useful instrument in the detection of aortoliac obstruction. Yao found the presence of a disturbed pressure/flow pattern at the ankle, combined with a pulsatile turbulent flow pattern (section 6.2.2.) recorded over the common femoral artery after exercise, indicative of a critical stenotic lesion of the femoral inflow tract (YAO 1970). NAYMAN (1974) came to the same conclusion. In a comparison of thigh pressure measurement (broad cuff) and a simple qualitative femoral artery velocity pattern analysis for the noninvasive diagnosis of aortoiliac stenosis the latter was found to be preferrable. A combined use of both tests was considered as probably the most useful screening test for the assessment of the functional severity of illiac artery disease (FARIS and JAMIESON 1975).

Based on measurements of the systolic thigh pressure and the aorto-tofemoral transit time (TT) and the calculation of the damping factor (DF) for the segment, a grading system applicable to the aortoiliac pathway was developed by FITZGERALD and CARR $(1975,1977)$. It distinguished between three groups of pathology, subdivided in a total of ten subtypes. Group I indicated progressive changes in the vessel wall with diffuse reduction of the lumen but without protruding obstructions and was characterized by only slight reduction of the femoral PI and by a DF, TT and thigh pressure within normal limits. Group II, the group with varying degrees of localized stenotic lesions, was characterized by a moderate fall in distal PI and some increase of the DF and a nearly normal TT and thigh pressure. In more severe obstructions also the pressure was lower than normal. Group III stood for total occlusion with various degrees of collateralization, and was defined by a low distal PI, increased DF and TT and a subnormal segmental pressure. In 80 aortoiliac segments, thus classified, an excellent correlation was found with the angiographic appearance: only in one case the segment was considered to belong to group III whereas the angiography showed only a severe stenosis; in all other segments a total agreement was found (FITZGERALD and CARR 1977). Since it was not specified whether the examined segments were affected singularly of were accompanied by distal obstructions, no conclusions are possible regarding the overall diagnostic value of this very complicated combined use of parameters.

FRONEK and co-investigators (1978) stressed the importance of a combined use of pressure measurement and femoral Doppler signal analysis in the evaluation of the arteries proximal to the groin. In 47 of $148 \mathrm{limbs}$ ( 31.8 percent) in which angiography confirmed ilac or common femoral artery occlusive disease a normal or even elevated upper thigh segmental pressure was found. Quantitative criteria of the femoral Doppler signal-peak velocity, deceleration and ratio of peak and mean velocity (sections 6.3.1. and 6.3.2.) - were thereupon considered also and in 42 of the 47 legs with an "erroneously" normal thigh pressure these parameters were abnormal. The authors called this a 
"pressure-velocity dissociation". In only 3.4 percent of all legs with significant proximal disease both normal thigh pressures and normal femoral Doppler signal parameters were found. So the use of pressure and signal analysis together could increase the diagnostic sensitivity from 68.2 to 96.4 percent. No figures about specificity and accuracy of the combined use of these parameters in diagnosing proximal obstruction could be deduced. Also a "reversed pressure-velocity dissociation" could be found in which a low thigh pressure was associated with only slightly abnormal velocity indices of the Doppler signal. This was the case in iliac stenosis with good collateralization. The conclusion of the study was that a combination of a low segmental thigh pressure index and significantly reduced velocity indices unequivocally confirmed the presence of a significant proximal stenosis with a poor local collateral flow and/or poor vessel patency at the site of measurement (common femoral artery).

\subsection{CONCLUSIONS}

At the present state of the art in the noninvasive diagnosis of aortoiliac obstruction with Doppler methods, one can only distinguish to some extent between significant and non-significant obstruction - i.e. more or less than 50 percent diameter reduction. The identification of different degrees of obstruction is still far from possible. Thigh pressure measurements have an established role in this diagnosis but the method is not very specific because concomitant superficial femoral artery occlusions interfere with it. Single level thigh pressure measurements, therefore, will always have a reduced specificity in the identification of aortoilliac stenosis. There are good reasons to believe that combined thigh and distal pressure measurement may better identify inflow obstruction to the leg because it identifies some of these superficial femoral artery obstructions making the method more specific for proximal lesions. The simple qualitative analysis of a femoral Doppler signal seems to have a diagnostic potential similar to thigh pressure measurements, which means that in about 20 percent of the patients a wrong diagnosis will be made. Quantitative Doppler signal analysis is a new technique of which the value for the diagnosis of aortoiliac obstruction is too scarcely studied to allow a judgement at this moment. The good linear relationship which could be found between the severity of aortoiliac obstructive lesions and the pulsatility index of the femoral artery justifies further investigations of this parameter, although at this moment the complexity of its calculation will be a handicap if the method has to compete with the simpler qualitative analysis or thigh pressure measurement. Damping factor, foot-tofoot and ECG related waveform transit times are hardly studied in the aortoiliac region. The combination of thigh pressure measurement and a characterization of the femoral artery Doppler signal seems to be a reliable indicator of the presence or absence of severe aortoiliac obstruction. Because one parameter may detect pathology which is missed by the other ("pressure-velocity dissociation") the combined diagnostic value will be better than that of each of the parameters used alone. An estimation of the relative value of the various difscussed tests is hardly possible because untill now the results are not reported umiformly in literature. The retrospective calculation of diagnostic accuracy, specificity and sensitivity of a published test is anly feasible in a small number of publications and the results obtained - like those presented in this chapter - can onlly be considered as a rough approximation of its validity. 


\section{EXAMINATIONS, MATERIAL, INSTRUMENTATION AND DATA ANALYSIS}

\subsection{GENERAL SET-UP OF THE STUDY}

Three major aspects are distinguishable in the present study each with its own technical and methodological approaches. The first is that of data collection which was performed in the setting of a surgical clinic. It consisted of the performance of routine physical vascular examinations, a combination of noninvasive ultrasonic Doppler tests and angiography in a series of patients with atherosclerosis of the lower extremities. The second part of the study comprised the processing of the material recorded during the clinical research phase. It included audio frequency spectrum analysis of Doppler signals and computerized analysis of their waveforms with calculation of a number of numerical waveform parameters. The facilities and knowledge for these techniques are usually not available in general hospitals which made cooperation with specialized outside institutes mandatory for this part of the study. The third part of the study was an elaborate statistical analys is of the numerous data resulting from the numerical working up of tests and Doppler signals.

\subsection{SELECTED NONINVASIVE ULTRASONIC DOPPLER TESTS}

Basically the Doppler tests used in the study were of three kinds: various segmental systolic pressure readings in the limbs, recording of Doppler signals along the arterial tree of the legs and determination of the transit time of the velocity wave along the iliac arteries. Systolic pressure measurements were performed in the arms and at the level of thigh and ankle to get an idea about the location of arterial obstructions in this way (section 5.5.4). At rest Doppler signals were recorded from the abdominal aorta, the common femoral artery and one of the foot or ankle arteries. The concept behind the selection of these sites was also that of a segmental identification of obstructions by means of observation of signal changes from aorta to common femoral artery and/or from the common femoral artery to the distal part of the leg. Aortic and common femoral signals were also recorded simultaneously for the determination of the aorto-to-femoral transit time of the velocity waveform.

An essential part of the examinations of the subjects in this study consisted of testing during hyperemia which was induced in two different ways. Pressure measurements were combined with treadmill exercise and Dopplet signal recording with post-occlusion hyperemia. Treadmill exercise was chosen because it provokes an ankle pressure fall of sufficiently long duration to allow quietly performed pressure readings and in addition gives a good impression about the general physical condition of the patient (see section 5.2.). The treadmill test with post-exercise ankle pressure readings served two goals in this study: the evaluation of the walking test itself as a means of grading the functional impairment of vascular patients and the study of post-exercise ank le pressure response types as a means of localizing obstructions anatomically (section 5.5.3). Ideally the response of Doppler signals to exercise which may give valuable information on arterial obstructions (YAO 1970 and section 8.4) 
should also have been recorded after the treadmill test. It would, however, have necessitated the use of more than one Doppler meter and more than one operator to correctly perform multiple simultaneous pressure readings and signal recordings within a relatively short time span under non ideal test circumstances. After a tiring walking test patients are often in pain, moving their legs while trying to find a comfortable position on the examination table. For this reason post-occlusion hyperema (thigh cuff occlusion) was preferred for hyperemic Doppler signal recording. The post-occlusion hyperemic reaction in legs with arterial obstructions is dellayed and prolonged in comparison to that in normal limbs as was shown in flow studies using venous occlusion plethysmography (HILLESTAD 1963a) and isotope technique (LASSEN et al 1964) and in Doppler flow velocity studies (FRONEK et al 1973a and section 2.2.6). Doppler signals of the common femoral artery were recorded one and three minutes after release of thigh occlusion. Based on the just mentioned studies about post-occlusion hyperemia, it was expected that in normal legs any changes in the Doppler signal due to increased flow velocities would have subsided within about one minute. In patients with obstructive arterial disease some differences with the signals at rest might still be perceivable one or even three minutes after cuff release. This could then possibly be used as a criterion for the degree of arterial obstruction.

A detailed description of the various Doppler examinations employed in this study is given in section 9.5 .

\subsection{POPULATION STUDIED}

In principle all patients admitted to the hospitital for angiography of the lower extremities, including the abdominal aorta and the pelvic arteries (aortography) were included in the study. No other formal selection criterion was employed. However, some very debilitated patients who were considered to be unable to undergo or perform all the tests were excluded. Some possible candidates were not examined for such practical reasons as temporary absence of the investigator or equipment defects. At a later stage of the study also the patients with abdominal ane urysms who came for aortography were excluded since the main area of interest of this study was the obstructive arterial lesion. At the time a patient was admitted to the study the decision to angiography had already been made by one of the vascular surgeons, independently of the aims and the progress of the study. The indication for angiography was only based on history, physical examination and ankle pressure measurements at rest. No other tests, like the ones described in this study, were awailable to the surgeon who made the decision. The indications for aortography which were already in use before the study started had not been changed for the purpose of this study. They were:

- Intermittent claudication considered severe enough to justify a vascular operation. The fist-choice approach to claudication was (and still is) a nonoperative one.

- Ischemic rest pain.

- Necrosis or ulcers due to far advanced arterial obstruction or due to emboli supposed to originate from aortoiliac or femoropopliteal pathology.

- Abdominal and peripheral aneurysms for which elective operation was indicated.

If on clinical grounds the surgeon was convinced that no important pathology 
had to be expected proximal to the common femoral artery, only a femoral needle angiography was performed. In that case the patient was not included in the study.

As a reference group healthy medical students, residents, young nurses and older people were included in the study. The older normals were either hospital employees without symptoms of cardiovascular diseases at their yearly medical check-up (history, physical examination, chest X-ray, ECG and biochemistry) or older patients meeting the same demands, who had been admitted to the surgical wards for diseases not related to the cardiovascular system.

The total number of patients in the study was 96 , of which 78 males (mean age $60.9 \pm 9.9$ years) and 18 females (mean age $57.5 \pm 15.4$ years). These figures clearly reflect the higher incidence rate of symptomatic peripheral vascullar disease in men than in women (section 2.1.4.). A total of 28 normal males and females were included in the study, of both sexes 14, with an equal age distribution. The young normals were 10 men (mean age $27.1 \pm 2.5$ years) and

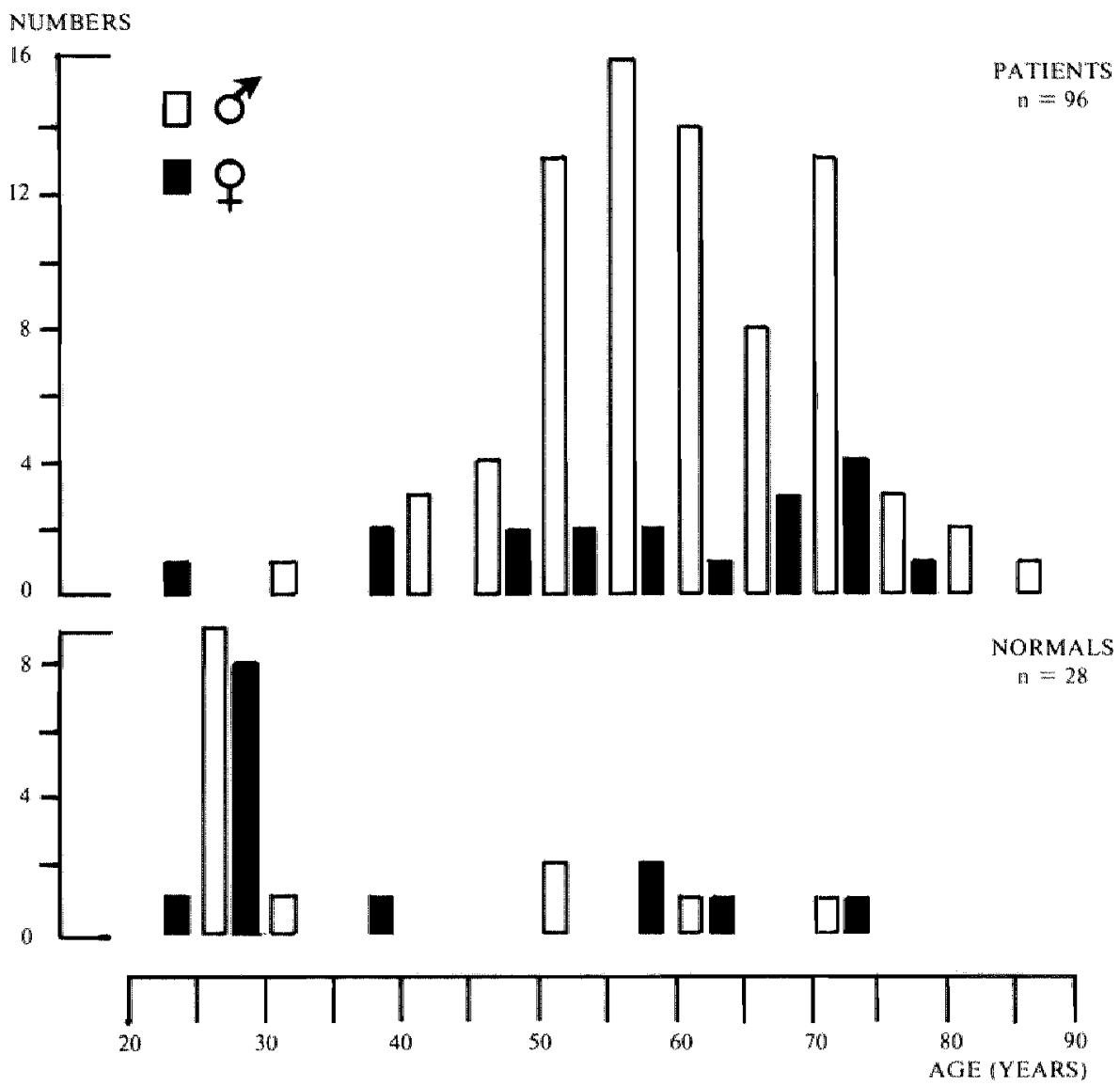

FIGURE 9.1

Age and sex distribution of the patients and the normal test subjects in the study.

The figure gives the total number of males and females in each age group of 5 years. The ages should be read as $20-24,25-29,30-34$ etc. 
10 women (mean age $27.5 \pm 3.7$ years). The older normals were 4 men (mean age $58.7 \pm 9.8$ years) and 4 women (mean age $62.2 \pm 7.3$ years). The age and sex distribution of the normals and the patients is given in Figure 9.1.

Four groups of patients could be distinguished as far as the reason for angiography and hence inclusion in the study was concerned: patients with intermittent claudication ("claudicants"); patients with ischemic rest pain in the feet ("rest pain"); patients with ischemic tissue necrosis of the lower extremities ("necrosis") and a group without spontaneous vascular complaints ("atsymptomatic"). For each of the patients' groups the total number of patients, the average age and the duration of the symptoms is given in Tabel 9.1. Twelve

TABLE 9.1.

Numbers of patients in the study with their mean ages, their symptoms and the duration of their symptoms.

\begin{tabular}{lccccc}
\hline & $\begin{array}{c}\text { SYMPTOMS } \\
\text { Claudication }\end{array}$ & Rest pain & Necrosis & A-symptomatic & TOTAL \\
\hline Number & 62 & 15 & 6 & 13 & 96 \\
\% of all patients & 64,6 & 15,6 & 6,3 & 13,5 & 100 \\
\hline Mean age (years) & 57,5 & 61,5 & 63,8 & 69,9 & 60,2 \\
$\pm \mathrm{SD}$ & 11,5 & 7,9 & 10,7 & 6,0 & 11,1 \\
\hline $\begin{array}{l}\text { Duration of the } \\
\text { symptoms }\end{array}$ & $10 \%$ & $60 \%$ & $83 \%$ & - & \\
$<1 / 2$ year & $21 \%$ & $27 \%$ & $17 \%$ & - & \\
$1 / 2-1$ year & $69 \%$ & $13 \%$ & $0 \%$ & - & \\
$>1$ year & & & & & \\
\hline
\end{tabular}

of the thirteen "a-symptomatic" patients had their examination because of aneurysmatic arterial disease (ten abdominal aneurysms, one common femoral artery aneurysm and one popliteal aneurysm). The remaining a-symptomatic patient was a woman, suspected of having anastomotic problems with her aortic bifurcation prosthesis. The majority of patients were claudicants with a long lasting history. There were no significant differences between the male and the lemale patients with regards to the relative distribution of symptoms or the average age. Of all patients 81 percent was still smoking or had stopped smoking less than half a year before; 28 percent of them were on an anti-hypertensive medical regime (dietary restrictions and/or drugs); 8 percent were on treatment for diabetes mellitus (diet and/or drugs); 55 percent had a positive family history for cardiac, cerebrovascular or peripheral vascular disease and 50 percent stated to suffer from cerebrovascular or cardiac disease concomitant with the symptoms for which they underwent the examination. Overall, these figures are in good accordance with the ones presented by JUERGENS and BERNATZ (1980) under the heading of "etiologic factors" of artherosclerosis of the extremities. 


\subsection{EQUIPMENT AND INSTRUMENTS}

\subsubsection{Doppler signal registration system}

All Doppler audio signals obtained with the intention to analyze them quantitatively or qualitatively were recorded on a stereo taperecorder(TEAC-A2300SD) without any pre-processing. The full forward and reverse Doppler frequency spectrum was thus preserved. At the moment of recording the audio signal could be listened to by the investigator by means of a stereophonic headphone or loudspeakers to check its quality. Simultaneously the sounds were processed through the two zero-crossing detectors - one for the forward and one for the reverse channel of the system - and separately displayed on an oscilloscope. If desired, the same signals could also be recorded on a chart recorder connected to the zero-crossers. The guidance of the probe-positioning hand with the help of these acoustic and visual signals proved to be very valuable throughout the tests. A diagram of the recording system is given on the left side of Figure 9.2. Two Doppler meters were used in the study (SONICAID model BV 380 (Sonicaid Ltd) one meter operating at a transmitter frequency of

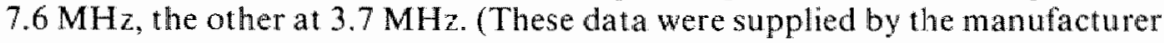
and checked in the Ultrasound Laboratory of the Biophysics Dept. of the University of Limburg. Maastricht, under supervision of A. Hoeks, M. Sc.). In these meters the separation between the forward and the reverse flow components is accomplished by phase-quadrature detection (section 4.2.3.). In the circuitry an outphaser as described by COGHLAN and TAYLOR (1978) was incorporated to allow spectrum analysis of the output. The focus-distance of the $7.6 \mathrm{MHz}$ probe as specified by the manufacturers and tested by the above mentioned Ultrasound Laboratory was $1 \mathrm{~cm}$ with an effective ultrasound beam

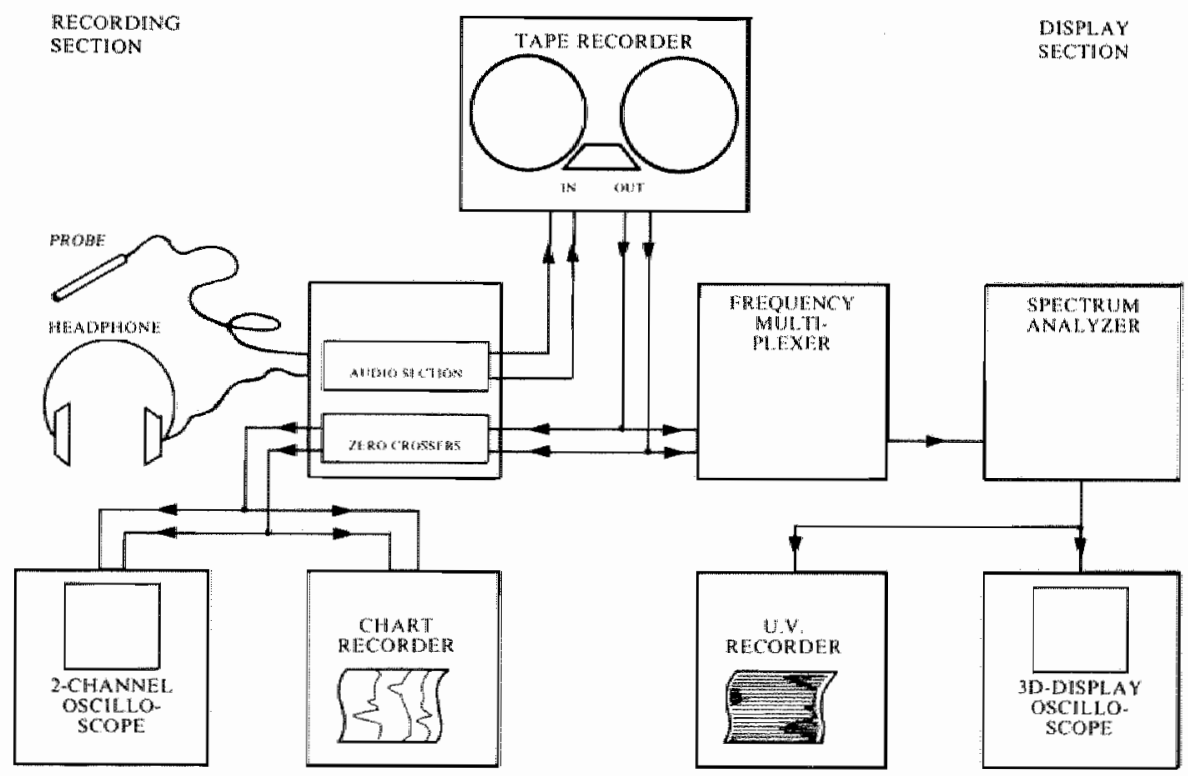

FIIURE 9.2

Equipment set-up for recording and displaying the Doppler signals and the auxiliary devices to monitor signal quality during recording. 
length of about $4 \mathrm{~cm}$ for the maximum minus $6 \mathrm{~dB}$ area (for definitions of "focus-distance" and "beam-length" see Figure 4.2). For the 3.7 MHz probe the focus-distance was $7.5 \mathrm{~cm}$. Both Doppler meters were incorporated in the recording systems, although only one meter is shown in Figure 9.2. Switches allowed any combination of forward and/or reverse signals from one or both meters to be selected for input into the taperecorder and furtheron into the display system.

\subsubsection{Doppler signal display system}

The aim of the recording and processing of the Doppler signals was to obtain a sonagraphic waveform display suitable for analysis. This was accomplished by submitting both the forward and the reverse Doppler frequency spectrum to audio spectrum analysis. It could be shown that the maximum frequency envelope of this sonagraphic display - which essentially has the same shape as the familiar waveform of electromagnetic flowmet ry - does not change its basic shape for a wide range of angles between the main flow direction and the ultrasonic beam (WOODCOCK et al $1972 \mathrm{~b}$ ). Other reasons to select audio spectrum analysis as the method of displaying the Doppler signals were the advantages of the method mentioned in section 4.5.3.

A single channel spectrum analyzer (NICOLET UA-500A) was employed. To enable the simultaneous analysis of the forward and reverse Doppler spectrum, both spectra were mixed to one single signal by means of frequency-domain multiplexing. The frequency multiplexer used for this study in conjuction with the spectrum analyzer was developed in the Ultrasound Laboratory of the University of Limburg, Maastricht, and has been described by RUISSEN (1980). (see Figure 9.3.). In this frequency multiplexer one of the Doppler spectra (e.g. the reverse) is modulated with a reference or carrier signal of a stable frequency

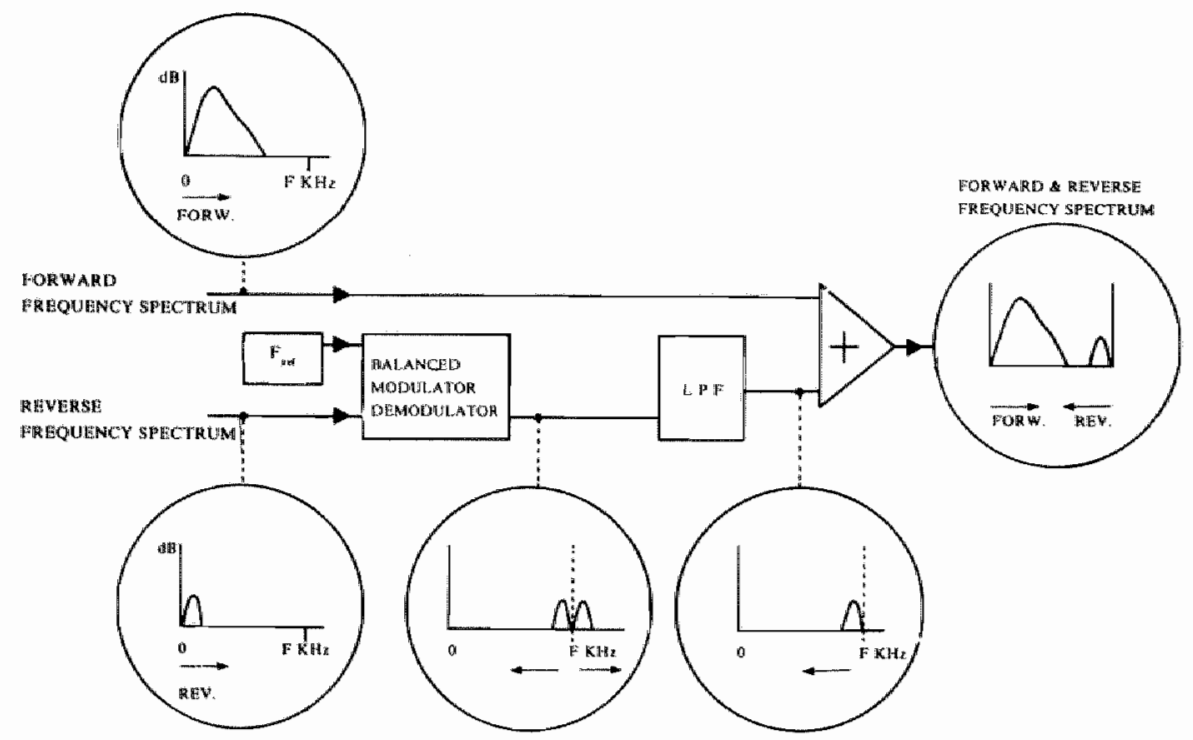

FIGURE 9.3

Principle of frequency multiplexing. 
$\left(f_{\mathrm{fef}}\right)$ in a balanced modulator-demodulator. The carrier frequency itself is suppressed in the modulator. The output of the modulator-demodulator is a frequency spectrum containing the original spectrum wich has been shifted to a higher frequency with $\mathrm{f}_{\text {ref }}$ (upper side band) plus the mirror image of the same spectrum below the $f_{r e r}$ (lower side band). The upper side band is eliminated by making the upper limit of the analysis range of the spectrum analyzer equal to $f_{\text {ref }}$ and by passing the signal through a low pass filter. The non-modulated other Doppler spectrum (e.g. the forward) is summated with the modulated signal. The resulting single signal contains all frequencies of both original signals, now projected in opposite directions. The zero scale of one signal is the maximum frequency scale of the other signal and vice versa. The selected carrier frequency determines the width of the frequency spectrum which can be passed through the multiplexer-analyzer circuit. The optional range in this study was $6,8,10$ or $12 \mathrm{KHz}$. The diagrammatic representation of the display system for the directional Doppler signals is given in Figure 9.2. It shows how the forward and reverse audio signals are channelled from the tape, on which they were recorded, through the frequency multiplexer to the spectrum analyzer. The sonagraphic result of this audio frequency analysis was displayed on a $3-D$ (three dimensional) oscilloscope and as a permanent record or sonagram via U.V. recording (HONEYWELL Visicorder) on high-speed, direct-print photographic paper (KODAK Linagraph) (see e.g. Figure 4.4). Since the system receives its input from a tape recorder which can be played-back at the very moment of recording, it can be considered to be real on-line display system.

In this study the signals were analyzed off-line some time after recording. This has the advantage that the tapes - if desired - could be played-back at one half of the speed $(9.5 \mathrm{~cm} / \mathrm{sec})$ which was used during recording $(19 \mathrm{~cm} / \mathrm{sec})$. This allowed to double the number of spectrum-analysis-runs made within the same period of physiologic signal recording.

\subsubsection{Channel separation of the Doppler system}

Since presence and characteristics of the reverse flow waveform of the Doppler signal play an important role in the qualitative and quantitative analysis of the signal, it is important that this signal is reliable. A possible cause of faulty reverse signals is cross talk of forwand signals on the reverse channel circuitry due to inadequate channel separation. A reason for this cross talk might be a deviation from the ideal $90^{\circ}$ phase difference within the phase quadrature detection system. A $5^{\circ}$ deviation may yield a $-20 \mathrm{~dB}$ cross talk (COGHLAN and TAYLOR 1978).

For the $7.6 \mathrm{MHz}$ Doppler meter used in the present study the manufacturer specified a phase error of $\pm 2^{\circ}(300 \mathrm{~Hz}-10 \mathrm{KHz})$ with a cross talk of $-30 \mathrm{~dB}$. The channel separation of the whole circuit from the Doppler meter $(7.6 \mathrm{MH} z)$ through the multiplexer down to the spectrum analyzer (see Figure 9.2) was tested electronically. To this purpose a "Doppler" signal was induced by excitation of the receiver of the Doppler meter by means of a signal from a frequency synthesizer (HEWLET PACKARD 3330B) fed to an induction coil which was wound around the Doppler probe. Differences between this signal and the oscillator frequency of the meter could be arranged in steps from 0.5 to $15 \mathrm{KHz}$. The power of the signals conveyed to the probe was chosen in such a way that the voltages measured at the audio outlet of the Doppler meter ranged from 40 to $200 \mathrm{mV}$ (rms). This range of artificial signal strengths was selected 
since outlet voltages of a variety of Doppler signals recorded under clinicall circumstances from normals and patients never exceeded $160 \mathrm{mV}$. By selecting signal frequencies above the oscillator frequency flow towards the probe was simulated. Any signal appearing on the reverse channel - the strength of which was measured with the amplitude indicator of the spectrum analyzer - was considered to be cross talk. The minimum cross talk which could be measured with this method was $-40 \mathrm{~dB}$. The maximum cross talk measured in the circuitry was $-22 \mathrm{~dB}$ for the strongest signal $(200 \mathrm{mV})$ at the lowest frequency $(0.5 \mathrm{~K} \mathrm{H} z$ ). For the more physiological signal strengths it was between $-25 \mathrm{~dB}$ and $-30 \mathrm{~dB}$ in the frequency range from $0-6 \mathrm{KHz}$ and below $-40 \mathrm{~dB}$ beyond these frequencies. This means that for the frequencies usually generated in physiological tests, the channel separation of the system is more than adequate (COGHLAN and TAYLOR 1978). Only for very strong signals of low frequency some cross talk between the channels may become visible.

\subsubsection{Sphygmomanometric equipment}

For sphygmomanometry a membrane manometer with an electrical inflation. pump and large size dial (SPEIDLER \& KELLER) was used. For a correct sphygmomanometric determination of systolic segmental pressures in the leg. cuffs should be used of which the width is related to the diameter or circumference of the limb at the site of measurement (section 5.1.). In this study a compromise solution was chosen by using cuffs of which the size was determined by the site of measurement irrespective of the individual circumference of the limb. This approach is an accepted one and adopted by all investigators whose studies were mentioned in Chapter 5 . It eliminates the need to measure the limb circumference and keep a variety of cuffs available. The widths of the cuffs in this study were such that in normal subjects the systolic pressures measured at the level of the thigh and the ankle would about equal the arm pressure (section 5.1.). Two types of non-tapered cuffs (ACCOSON) were employed. A standard arm cuff of which the textile band measured 51 by $14 \mathrm{~cm}$ and the inflatable rubber bag 22 by $12 \mathrm{~cm}$; and a broad cuff measuring 76 by $20 \mathrm{~cm}$ (band) and 51.5 by $18 \mathrm{~cm}$ (bag). The small cuffs were used in arm and ankle pressture measurements, the wide cuffs for measurements at the thigh.

\subsection{EXAMINATION PROTOCOL AND DESCRIPTION OF TESTS}

All patients underwent the various tests within three days before their angiography, with only two exceptions in which there was an interval of only a few days more. All tests were performed during a single uninterrupted session and were conducted in the same examination room which was kept at approximately the same temperature level during all cessions. The mean room temperature during the examinations was $23.0 \pm 1.5^{\circ} \mathrm{C}$. The test sequence was strictly the same in all examinations. All tests were performed by the author personally, single-handed. The patients were brought from the wards to the examination room in a wheelchair while the normals came walking. All had refrained from smoking for at least one hour. Before the start of the tests all subjects rested in a supine position on the examination table during 20 minutes, dressed only in their underwear. After that, the peripheral pulses in the groin (common femoral artery), in the knee (popliteal artery), at the dorsum of the foot (dorsalis pedis artery) and behind the medial malleolus of the ankle 
(posterior tibial artery) were palpated. The pulses were quantified using a score from 3 to 0 ( 3 = normal, straight away palpable pulse; 2 = clearly diminished, but easily discernible pulsation; $1=$ hardly palpable pulse, often only found after at prolonged search for it; $0=$ no pulsation to feel). Also the presence of bruits in the aortoiliac and upper leg region was searched for with a stethoscope and the result of this examination was recorded as "absent" or "present".

Next, the systolic and diastolic blood pressures in both arms were determined using the classical Riva-Rocci method, followed by the systolic blood pressure measurement at upper thighs and ankles using the Doppler method (section 5.1.). For the thigh pressure determination the broad cuff was applied to the upper leg as proximal as possible, while the return of the blood flow was determined with a probe above the posterior tibial or dorsalis pedis artery. Sometimes it was necessary to position the probe at knee level because of poor distal Doppler sounds. For the ankle pressure measurement the standard cuff was applied just proximal to the ankle malleoli and the pressure was measured once with the probe directed towards the posterior tibial artery and once towards the dorsalis pedis artery. Then, the directional Doppler signals from the abdominal aorta, both common femoral arteries and the arteries of the foot were recorded on the tape recorder. Either the posterior tibial or dorsalis pedis signal was recorded depending on which of the arteries gave the better signal (highest frequencies). The anatomical landmark for the aorta was a point 2 fingers proximal and to the left of the umbilicus. For the aorta the $3.7 \mathrm{MHz}$ meter was used, for all other arteries the $7.6 \mathrm{MHz}$ device. The common femoral artery was located by searching for the characteristic sound of the femoral vein in an area $1-3 \mathrm{~cm}$ distal to the inguinal ligament and then moving the probe about $1 \mathrm{~cm}$ more lateral until an arterial sound was heard. The posterior tibial artery was monitored in the groove dorsal to the medial malleolus of the ankle and the dorsalis pedis artery at the dorsum of the proximal part of the foot which was kept in a relaxed dorsiflexion. Of all signals at least ten successive pulses were recorded. To reach an optimal signal quality the probe-vessel angle was changed by moving the probe until the loudest possible signal was heard and the zero crossed signal had reached the highest amplitude on the oscilloscope. Next, followed the simultaneous recording of the forwand components of both the aortic signal and the common femoral artery signal on the tape recorder for off-line determination of the aorto-to-femoral pulse transit time. Again at least 10 successive heart beats were recorded, for the right as well as the left side. Then, hyperemia was induced in both legs by simultaneously deflating thigh cuffs which first had been kept inflated at a pressure of 250 $m m \mathbb{H g}$ during 5 minutes. After the fast release of both culfs, the signals of the common femoral arteries were recorded after 1 and 3 minutes, always the right side first; then the left side. If any of the hyperemia signals could not be recorded within half a minute after the time interval indicated on the protocol the measurement was excluded from further analysis. At the end of this hyperemia test the subject was kept 5 more minutes resting on the examination table to allow any hyperemia still present to dissapear completely (section 5.2.). In this period no tests were performed. After this procedure a standard walking test and post-exercise ankle pressure measurements were performed. Walking was done on a horizontal treadmill which was speeded up from stand still to $4 \mathrm{~km} / \mathrm{hr}$ in as short a time span as the person under investigation could tolerate. All further time measurements were started from the moment that full speed was reached. The tested person was allowed to maintain equilibrium by holding the 
two sidebars of the treadmill. Patients were not monitored electrocardiographically since in the period in which the clinical tests were performed the need to do so was not yet as generally recognized as it is nowadays. Patients were instructed to mention any angina pectoris immediately because this would be at criterium to stop the test at once. Other criteria to stop the test were severe dyspnea, severe claudication pains or any other complaint for which the patient wished to stop. If no such complaints were mentioned the test was stopped after 5 minutes (time criterium). The localization of the claudication pain, if any, was recorded, as well as its time of onset (claudication time) and the time at which walking was stopped (maximum walking time). Immediately after cessation of walking the tested person returned to the supine position on the examination table to allow first a search for the presence of arterial bruits in the aortoiliac region and upper legs. This was immediately followed by repeated brachial and ankle systolic blood pressure measurements (Doppler method). In each leg the ankle/foot artery was used for measurements which had the higher bloodpressure at rest. The arm with the highest systolic pressure at rest was used for measurement of the systemic blood pressure after exercise. The sequence of measurements was always: arm, right ankle, left ankle. Each series of measurements had to be performed within 1 minute to be accepted for further analysis. Measurements were made $1,3,5,10$ and 15 minutes after the cessation of walking.

\subsection{ANGIOGRAPHY}

The angiographic examination of the patients was usually performed as a onedirectional (p.a.) translumbar aortography (TLA) under general anesthesia or lumbar epidural block anesthesia with the patient in the prone position. Each angiography series consisted of a total of $14-16$ exposures (film size $35 / 35 \mathrm{~cm}$ ), covering the whole vascular tree from the infrarenal aorta down to the ankle or foot arteries in 4 or 5 partly overlapping fields. Frequently more than one series was taken with a different timing of the exposures to ensure an optimal delineation of the pathology. If the peripheral arteries were poorly or not visualized by the TLA, the patient was turned to the supine position and one or more additional series were made after retrograde contrast injections through a needle in the common femoral artery. The standard amount of contrast solution (Urografin $76 \%$ ), injected at a rate of $8 \mathrm{ml} / \mathrm{sec}$, was $40 \mathrm{ml}$ for a TLA series and 20 $\mathrm{ml}$ for a femoral angiography. Exceptionally, a Seldinger transfemoral or a transbrachial technique was used for special reasons.

\subsection{DATA PROCESSING}

\subsubsection{Numerical description of the angiogram (Angioscore)}

All angiograms were reviewed by the author a few months after the date of angiography. At that moment the data of the other tests had not yet been analyzed and may be considered as having been unknown to the reviewer. At a later date a second revision of all the X-rays, with the intention to confirm or correct the records of the first review, was performed by the senior surgeon of the vascular team (F.E.E. Vermeulen, M.D.) who had no knowledge of the results of the noninvasive tests.

All angiograms were scrutinized, segment by segment, for the presence of 
obstructive lesions. An obstruction could be either a stenosis or an occlusion. The definition of stenosis was as given in section 3.5.2. The degree of stenosis was expressed as a stenosis percentage. This was determined by measuring the narrowest remaining diameter within the stenosis and the pre-stenotic vessel diameter with a ruler. The difference between these two diameters was expressed as the percentage of the pre-stenotic diameter. If no real pre-stenotic segment was present - as for instance in stenoses right at the iliac bifurcation or the origin of the profunda femoris artery - the post-stenotic diameter at a site which appeared most normal was used as the reference value, preventing measurement in any post-stenotic dilatation. In the case of multiple stenoses in a single segment the severest stenosis was measured to characterize that segment. An occlusion was defined as a 100 percent stenosis or total obstruction of the vessel lumen.

The percentage diameter stenosis was separately measured for the following segments:

- abdominal aorta; from the origin of the renal arteries down to the artic bifurcation.

- common iliac artery; from the aortic bifurcation down to the iliac bifurcation or origin of the internal iliac (hypogastric) artery.

- external iliac artery; from the iliac bifurcation down to the level of the inguinal ligament.

- common femoral artery; from the inguinal ligament to the origin of the profunda femoris artery.

- origin of the profunda femoris artery; the first three centimeters of that artery.

- superficial femoral artery; from its origin at the femoral bifurcation down to the level of Hunter's adductor canal.

- popliteal artery; from Hunter's canal down to the first major branch below the knee, usually the anterior tibial artery.

- occlusions in the first few centimeters or non-visualization of any of the three lower leg arteries (anterior tibial artery, posterior tibial artery, or peroneal artery) was also recorded.

In view of the aims of this study the vascular tree was divided into an "inflow" and an "outflow" section. The inflow section included the aortic, common iliac, external iliac and common femoral artery segments. The outflow section was made up of the superficial and deep femoral artery, the popliteal artery and the crural or lower-leg arteries. The right and the left side of the arterial system were analyzed separately, which had as a consequence that the aorta was a common segment shared by both the right and the left inflow section. The inflow section and the outflow section were each described by one single figure, a so called angioscore. This angioscore was related to the degree of (angiographic) obstruction in that section. For the inflow section - equivalent to the aortoiliac pathway which is the area of interest of the study - the angioscore was equal to the highest percentage diameter stenosis of the four segments of which it was composed. It thus could get any value between zero (for an aortoiliac segment without obstruction) and 100 (in case of an occlusion somewhere in its course). For the inflow section also the segmental localization of any aneurysm was recorded for later correlative studies.

The angioscore used for the inflow section was not applicable as such to the outflow section. For the outflow section the score was based on the relative contribution to total volume flow in the stem arteries of the leg by the deep 
femoral, the superficial femoral, the popliteal and the crural arteries. In this system the superficial and the deep femoral arteries were equally important for the score. Both are of about the same calibre at their origin (VOLLMAR 1975, p. 265) and accept equal parts of the flow volume supplied by the common femoral artery (STRANDNESS and SUMNER 1975, p. 210). Others - using a different scoring system - also attributed the same weight to both these arteries in their system (CHAMBERLAIN et al 1975). The popliteal artery accomodates about one-lhird of the common femoral artery flow and each of the crural arteries about one-eighth as can be deduced from a summary of results of volume flow studies in the limb using different invasive and noninvasive techniques. (STRANDNESS and SUMNER 1975, p. 210). An occlusion of the proximal twothird of the popliteal artery in conjunction with an occlusion of the superficial femoral artery is a serious impediment to flow to the leg. The profunda femoris artery can only perform adequately as a collateral if a patent proximal popliteal artery is available as "receiver segment" ("Empfänger Segment", VOLLMAR 1975 p. 265).

On the analogy of the inflow score an obstruction score of the outflow section was developed with a scale from zero (no obstructions) to 100 (most severe degree of obstructive lesion).

In case of isolated occlusions the outflow scores were as follows:

- for the origin of the profunda femoris artery = score 50

- for the superficial femoral artery $\quad=$ score 50

- for the popliteal artery $\quad=$ score 33

- for each of the lower leg arteries at their origin $=$ score 8

In case of an "solated stenosis this was given a fraction of the corresponding occlusion score according to the following system:

- stenosis $\leqslant 50$ percent $=0$ percent of occlusion score

- stenosis $51-75$ percent $=40$ percent of occlusion score

- stemosis 76-95 percent $=80$ percent of occlusion score

- stenosis $>95$ percent = score as for an occlusion.

In the case of sequential obstructions in the superficial femoral, popliteal and crural arteries the highest score counted for the whole of that pathway. An exception was the combination of an occlusion in the superficial femoral artery with an occlusion of the proximal popliteal artery which, because of its serious character, always was attributed a score of 80 . In the case of a combination of lesions in the superficial femoral - popliteal - crural pathway on the one hand and the profunda fenoris artery on the other hand, the scores of each of these partallel systems were added to each other.

To allow a comparison of test parameters with the angiograms six angiographic classes (Figure 9.4.) were distinguished. The classification corresponds to the presence of hemodynamically significant arterial obstructions with a diameter stenosis of $>50$ percent (see 3.5.2.2.). The six different classes are:

\section{ANGIOCLASS 0}

This classification was assigned to both legs of the normals who had no angiography. It is based on a hypothetical in-and outllow score of zero.

\section{ANGIOCLASS I}

The anglograms of this class had an inflow score $\leqslant 50$ and an outflow score $\leqslant 50$. 
ANGIOCLASS 2

The angiograms of this class had an inflow score $\leqslant 50$ and an outhow scote $>50$.

\section{ANGIOCLASS 3}

The angiograms of this class had an inflow score $>50$ and an outflow score $\leqslant 50$.

\section{ANGIOCLASS 4}

The angiograms of this class had an inflow score $>50$ and an outlow score $>50$.

\section{ANGIOCLASS 5}

This classification was given to angiograms of which either the inflow score or the outflow score or both could not reliably be determined.
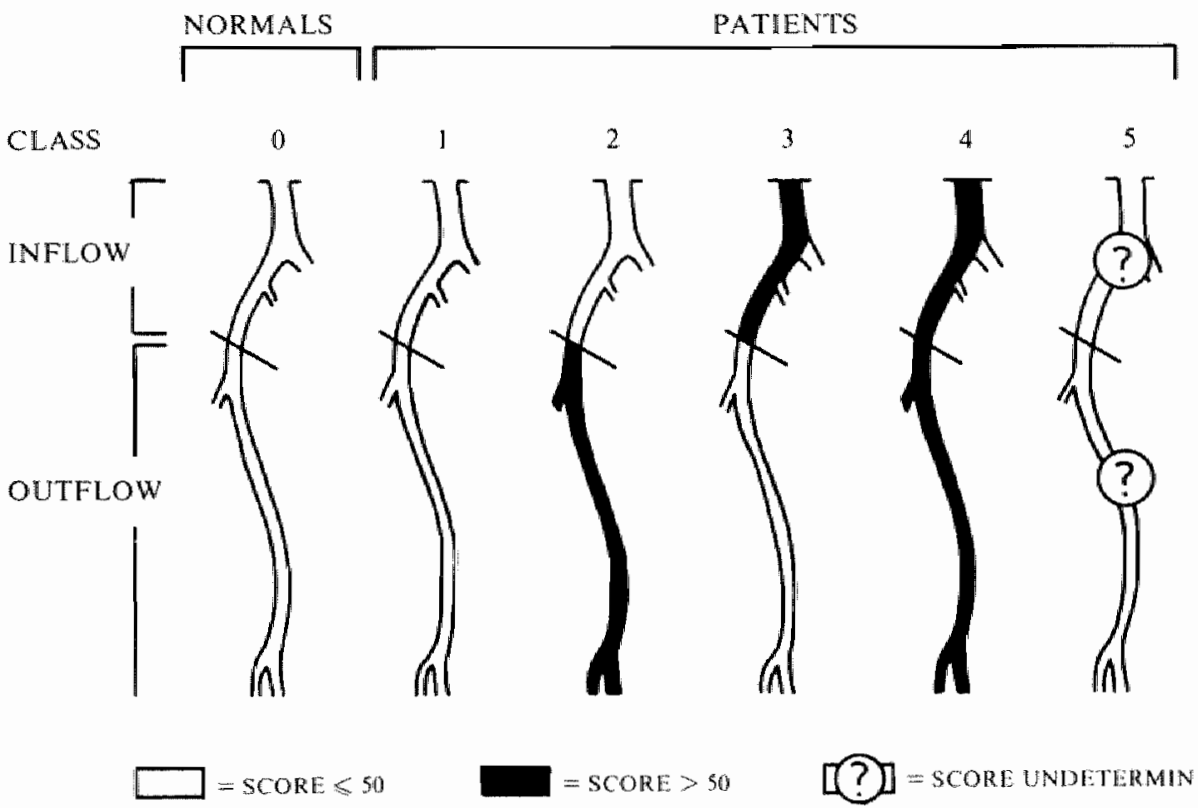

FIGURE 9.4

Angiographic classification of legs.

\subsubsection{Pressure data analysis}

All segmental systolic blood pressures at the leg, obtained at rest or after exercise, were expressed as a pressure index by dividing them by the corresponding highest arm pressure (section 9.5.).

\subsubsection{Analysis of post-exercise pressure indices}

The response of the ankle pressure indices to exercise was classified on the basis of three values: the pre-exercise index, the lowest post-exercise index and the index obtained 10 minutes after cessation of exercise. With the help of these 
criteria 6 types of ankle-index-response curves were defined, characterized by the resting level as well as profoundness and duration of the post-exercise pressure index fall, if any. This classification resembles the one published by GAY and ROBERTS (1979). The six response types are schematically represented in Figure 9.5. in which also the definition of each of the types is given.

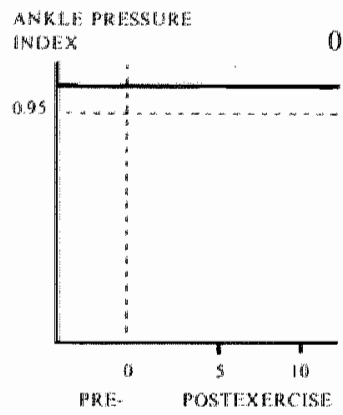

0
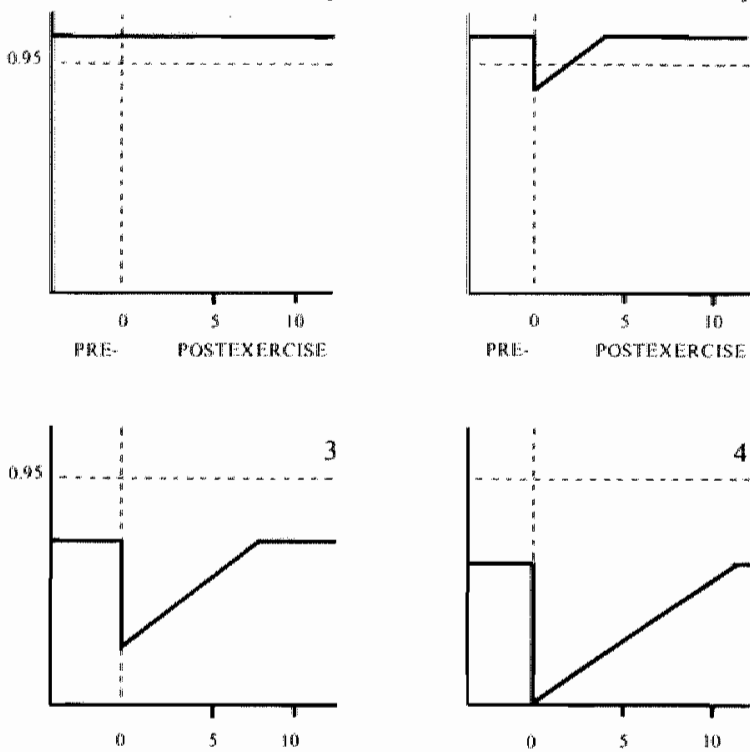

1
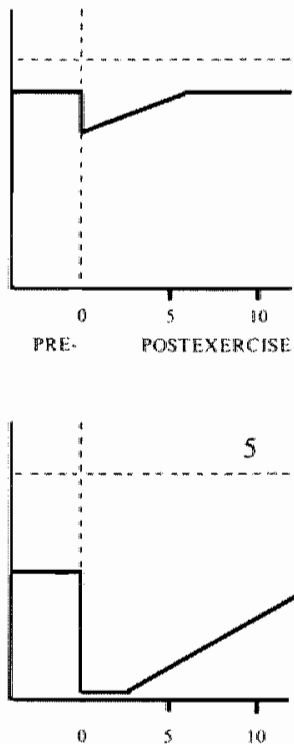

TIME IN MINUTES

FIGURE 9.5

Schematized response curves of the ankle pressure indices after treadmill exercise.

On the abscissa the time in minutes, on the ordinate the ankle pressure index. The vertical dotted line indicates the end of the exercise.

Definition of types:

Type $0=P_{p} \geqslant 0.95$ and $P_{\min } \geqslant 0.95$

Type $\mathrm{I}=\mathrm{P}_{\mathrm{p}} \geqslant 0.95$ and $\mathrm{P}_{\min }<0.95$

Type $2=\mathrm{P}_{\mathrm{p}}<0.95$ and $\mathrm{P}_{\mathrm{min}} \geqslant 0.75 \mathrm{P}_{\mathrm{p}}$

Type $3=\mathrm{P}_{\mathrm{p}}<0.95$ and $0.25 \mathrm{P}_{\mathrm{p}} \leqslant \mathrm{P}_{\min }<0.75 \mathrm{P}_{\mathrm{p}}$

Type $4=\mathrm{P}_{\mathrm{p}}<0.95$ and $\mathrm{P}_{\text {man }}<0.25 \mathrm{P}_{\mathrm{p}}$ and $\mathrm{P}_{10} \geqslant 0.8 \mathrm{P}_{\mathrm{p}}$

Type $5=P_{p}<0.95$ and $P_{\text {min }}<0.25 P_{p}$ and $P_{10}<0.8 P_{p}$

$\left(P_{p}=\right.$ Pre-exercise ankle pressure index; $P_{m i n}=$ lowest post-exercise ankle pressure index; $P_{10}=$ ankle pressure index 10 minutes post-exercise)

\subsubsection{Transit time measurement}

The time delay between the arrival of the aortic and the common femoral systolic velocity pulse was measured from the sonagrams (foot-to-foot distance) 
on which both signals were displayed simultameously (section 7.2.). This time delay or transit time was expressed in milliseconds (ms). To this purpose the tapes with the recorded signals were replayed at half the recording speed which made it possible to analyze each signal artificially at twice the normal analysis speed available in the spectrum analyzer or 250 analysis sweeps for each second of the real physiological signal. By displaying these sweeps on the UV recorder, set at a paper speed of $5 \mathrm{~cm} / \mathrm{sec}$, the distance between two successive sweeps on the obtained sonagram was 0.4 millimeter, representing $4 \mathrm{~ms}$ of physiological signal. The minimum transit time reasonably measurable with this method thus was $4 \mathrm{~ms}$. The distance between the foot of the aortic upslope and the foot of the femoral signal was measured with an instrument specially constructed for this purpose. It consisted of a transparant ruler which could be positioned on the sonagram exactly perpendicular to the time axis through the footpoint of the aortic signal and crossing the opposite zero base of the femoral signal. The distance between this crossing point and the foot of the upslope of the matching femoral signal was then measured by means of a microscale (intervals of 0.1 millimeter) read with the aid of a magnifier ( $8 x)$, all built-in in the measuring device. At least five successive beats were measured and averaged to obtain the transit time for one side.

Since it had been claimed that "normalization" of the transit time would improve the diagnostic potential of this parameter (sections 7.2. and 7.4.) also the normalized transit times were calculated for further correlative studies. For this the observed transit time was divided by the "normal" or "expected" time. This normal transit time can be read from a diagram published by GOSLING and KING (1974b), which was derived from measurements on healthy femoropopliteal segments with a length of $40 \mathrm{~cm}$. The diagram shows the relation between normal transit time, age and mean blood pressure. To obtain the "normal" aorto-femoral transit time half the value from the diagram was taken to compensate for the shorter length of that pathway (approximately 20 $\mathrm{cm})$. Since no experimental data are available on the normal aorto-femoral pulse transit times corrected for age and blood pressure, this method was used by Gosling himself and the outcome was found to be reasonable well in agreement with aortograms (GOSLING and KING 1978).

\subsubsection{Doppler sonagram waveform analysis}

\subsubsection{Qualitative analysis of the Doppler sonagram}

To describe qualutatively the waveform shape of the sonagram envelope the classification according to Thulesius, as described in section 6.2.2. and in Figure 6.1., was used. This classification is based on the presence or absence of a reverse wave and the height (or maximum frequency) of the monophasic signals. Because no definition of "high" or "low" frequency was given in the original publications of Thulesius these frequencies had to be defined. Based on the analysis of the lowest systolic peak frequencies of a small series of signals recorded from normals, the separation between high and low frequency for the Thulesius classification was established at $2 \mathrm{KHz}$ for the femoral signals and at 1 $\mathrm{KHz}$ for the ankle signals.

\subsubsection{Quantitative or numerate analysis of the Doppler sonagram}


The sonagrams were digitized for further computerized processing with the aid of a graphic tablet (TEKTRONIX 4953) connected to the computer. Separately the maximum frequency envelopes of the forward and the reverse flow waveforms of the sonagrams (see Figure 4.4) were traced one after the other by multiple touches with the special graph pen of the tablet, thus introducing the $x$ (time axis) and $y$ (frequency) coordinates of the envelopes into the computer. Of each sonagram three full pulse cycles were digitized. All x coordinates were nonuniformly sampled. The onsets of the systolic upslopes (foot points) of the three sampled pulse cycles and the foot point of the fourth systole (indicating the end of the third cycle) were indicated separately. A maximum of 245 coordinates could be sampled to describe the forward plus the reverse sonagram envelopes. In order to facilitate further calculations, a simple linear interpolation program was applied to these data to obtain a uniform sampling along the time axis at a rate of 1 sample every $10 \mathrm{~ms}$. After the process of interpolation any negative $x$ coordinate obtained during digitization of the forward curve and positive $y$ coordinate abtained during digitization of the reverse curve was made equal to zero. This made the exact tracing of zero frequency segments of the sonagramwhich surely would have introduced errors - unnecessary. Long zero-level sections could be indicated by two well separated coordinates with negative $y$ values and $x$ values indicating the beginning and the end of the segment. This procedure also lleft more coordinates from the maximum of 245 to be used for a detailed outline of the rest of the waveform. The digitized "curves" were filtered or smoothed with the aid of a 7 -points parabolic fit procedure. A plot program for the graphic reproduction of the data was available which allowed the visual comparison of the original sonagrams and the "curves" available in the computer.

\subsubsection{2b. Selection and calculation of numerate Doppler signal parameters}

The digitized sonagraphic waveforms were used for the computerized calculation of a variety of numerate signal parameters. If one considers sonagram waveforms and tries to determine which aspects make one waveform look different from another they appear to be such as: the presence or absence of reverse flow, the amplitudes of the curves, the steepness of the slopes and the fact that in some curves relatively long periods of zero level forward flow signal are found while in other curves forward flow is present during the entire pulse cycle. The numerical parameters of the Doppler signal waveform used in this study are based on these considerations. In the selection of the parameters the eventual feasability of parameter measurement and/or calculation without the use of a computer was also considered an important aspect.

The numerical Doppler signal parameters used in this study are:

MAXFF = Maximum or peak frequency of the forward flow envelope.

MEAN FF = Mean level of the forward flow envelope only, averaged over the entire pulse cycle and calculated by dividing the sum of all $y$ coordinate values within the cycle (including $y=0$ ) by the number of the coordinates.

MAX REV = Maximum level of the reverse flow envelope. This parameter has a negative sign in case a real reverse llow is present. When no 
reverse flow is present the parameter obtains the value of the minimum level of the forward curve.

PI = Pulsatility Index. See section 6.3.3. and Figure 6.3. for its definition.

$\mathrm{R} / \mathrm{E}=$ Ratio of MAX REV/MAX FF

$\operatorname{UST}(\mathrm{H})=$ Upslope time ("Hand" measurement). The time span between the onset of the systolic upslope of the forward curve as indicated by hand during digitization of the curve (section 9.7.5.2a) and the occurence of MAX FF.

UST(DER) = Upslope time ("Derivative" measurement). For the determination of this time interval the computer fitted a straight line through 5 points around the point at the systolic upslope of the forward curve where the slope was at its steepest. (=where the first derivative of the curve reached its maximum). The onset of the upslope is then defined as the point where this straight line crosses the zero axis. The UST(DER) is the time interval between this onset of the upslope and the time of MAX FF.

DST $\quad=$ Downslope time (Derivative measurement). The end of the first (systolic) forward curve is found by fitting a straight line through five points around the point at the descending $\operatorname{limb}$ of the systolic waveform where the slope is at its steepest (or where the first derivative of the downslope curve reaches a minimum). The intersection point of this line with the zero axis is taken as the end of the systolic curve. The DST is the time interval between the time of MAX FF and this systolic end point,

NULL $=$ Null fraction. The fraction of the total cycle duration for which the amplitude of the forward curve is cero.

In Chapter 6 signal parameters like PI, the R/F, the MAX FF, the MAXREV and the UST and DST or parameters very similar to these ones were discussed. The parameters DST and UST (DER) can hardly be calculated without a computer and the same applies to some extent to the calculation of the PI (see section 6.3.3). The other parameters might easily be measured by hand. For the DST it was difficult to think of a substitute suitable for hand measurement because of the often poorly defined end point of the downslopes of many waveforms. The upslope on the other hand is usually well circumscribed and allows hand measurement (UST $(H)$ ). The value of the parameter NULL indicates the degree of (dis)continuity of the Doppler signal. It should be recognized that a zero level signal does not necessarily mean the absence of flow but may also be the result of a filtering process within the Doppler equipment (section 4.2.1).

All parameters were determined for three uccessive pulse cycles and their average was used in further statistical and correlative studies.

\subsection{STATISTICAL ANALYSIS METHODS}

In order to study the dicriminating power of the numerous clinical and Doppler signal parameters, an interactive program for pattern recognition and analysis, named ISPAHAN, was used (GELSEMA 1981). This system greatly facilitates the analysis of data and provides classifiers based on a variety of classification strategies. The first step in the analysis of the data was the performance of twotailed Students' t-tests to select the object parameters which differed sig- 
nificantly in their mean values for various a priori defined groups or classes of objects. For an n-group problem this test was performed for all individual parameters and for each possibte combination of two out of the n groups. Only those parameters which had a significant difference between their mean values for any of the combinations of two a priori groups were considered for further analysis. The parameters which passed the t-test were ranked according to their discriminating value. To this purpose both the matrix of the sum of squares and sum of cross-products for the whole population of objects (W) and the so-called pooled matrix of the sum of squares and sum of cross-products of a priori groups (P) were computed. From these matrices the variance - covariance matrix as well as the pooled variance-covariance matrix could be computed by dividing the elements of matrix W and $P$ respectively by the degrees of freedom. As the most discriminating parameter the one was considered for which the ratio $F_{j}$ of the corresponding diagonal elements of the matrices $W$ and $P$ $\left(F_{j}=W(j j) / P(j j)\right)$ was maximal. Out of the remaining parameters again the most discriminating one was selected and so on until all parameters were ranked. Detaills of this procedure were described by COOLEY and LOHNES (1971). The principle of the method can be summarized as follows. Whenever a total of $a$ parameters had been ranked and a number of $b$ parameters still remain to be ranked, the residual matrices $\mathrm{Wb}$.a and $\mathrm{Pb}$.a are computed. When matrix $\mathrm{W}$ is partitioned as

$$
W=\left[\begin{array}{ll}
W a a & W a b \\
W b a & W b b
\end{array}\right] \text {, the residual matrix } W b a=W b b-W b a . \text { Waa } W a b .
$$

A similar expression is obtained for Pb.a. For each of the $b$ not yet ranked parameters again an $F_{1}$-value is computed as follows:

$$
F_{j}=\frac{(W b \cdot a(i j)-P b \cdot a(i j)) /(N-1)}{P b \cdot a(i j) / L-N-a)}
$$

where $\mathrm{N}=$ number of a priori groups and $\mathrm{L}=$ total number of objects to be classified. The parameter with the highest $F$-value explains most of the residual variance and hence had the largest additional discriminative power and is ranked accordingly.

In the present study the objects to be classified were the legs of patients and healthy subjects. The a priori groups or classes were the angiographic classes as described in section 9.7.1 or combinations of these classes. Starting with the most discriminative parameter and adding in a stepwise manner each time the next best parameter, classification rules were computed using the Fisher discriminant, which is a two-group classifier. The $c$ selected parameters used to classify the legs can be represented by a vector $X$ and the means of the parameter vectors of both a priorigroups to which the legs should be assigned as $M 1$ and $\underline{M}$ 2 respectively. One can find a vector $\underline{V}$ in the $c$-dimensional parameter space such that for the projections of the objects of the two a priori groups on that vector, the difference between their means is maximized relative to the pooled variance of the projections. In other words one has to find a vector $V$ such that the function $f(V)$ of this vector is maximal: 


$$
f(\underline{V})=\frac{\underline{\underline{V}} \cdot(\underline{M} 1-\underline{M} 2)(\underline{M} 1-\underline{M} 2)^{t} \cdot \underline{V}}{\underline{V}^{\mathrm{l}} \cdot S \cdot \underline{V}}
$$

where $S=$ the pooled variance covariance matrix in the $c$-dimensional space. $\underline{(V)}$ is maximal for $\underline{V}=S^{-1} \cdot(M 1-M 2)$ as was shown by DUDA and HEART (1973).

An object is assigned to one class (class 1 ) when $V^{6} . X<$ Vo and to the other class (class 2) when $\underline{\mathrm{V}} . \underline{\mathrm{X}}>\mathrm{Vo}$.

Vo is a threshold which is defined by

$$
V_{0}=\underline{V}^{i} \cdot M \underline{M}+\frac{\underline{V}^{i} \cdot S_{1}-\underline{V}}{\underline{V}^{i} \cdot S_{2} \cdot \underline{V}} \frac{V^{i} \cdot M 2-\underline{V}^{i} \cdot M}{2}
$$

in which $S_{1}$ and $S_{2}$ are the variance-covariance matrices for classes 1 and 2 respectively.

For the present study the Fisher discriminant analysis was also used in n-class problems $(n>2)$. To this purpose Fisher discriminants were computed for all combinations of two classes and each time the object was assigned to one of these two classes on the basis of the discriminants. After finishing this procedure for all $n$. (n-1)/2 possible combinations of a priori classes the object was finally classified in the a priori class to which the object had been directed most frequently during the intermediate classification processes.

The results of the classification procedures were expressed as percentages correct classifications. In the case of simplification of the classification procedure to a two-classes problem it was possible to calculate not only the proportion of correct classifications (accuracy) but also the diagnostic specificity and sensitivity of the noninvasive tests compared to angiography. The specificity is the proportion of true negative classifications, the sensitivity is the proportion of true positive classifications. Accuracy, specificity and sensitivity were calculated according to the use of the "decision matrix" as described by VECCHIO (1966) and MCNEIL et al (1975) and presented in Figure 9.6 (see also Chapter 8).

TEST RESULTS

ANGIOGRAPHY

$$
\begin{aligned}
& \text { ACCURACY }=\frac{a+d}{a+b+c+d} \times 100 \% \\
& \text { SPECIFICITY }=\frac{a}{a+b} \times 100 \% \\
& \text { SENSITUVITY }=\frac{d}{a+d} \times 100 \%
\end{aligned}
$$

FIGURE 9.6

Decision matrix.

In order to obtain optimal threshold values for results of noninvasive tests selected for a classification of legs in a two-classes system, a so-called Receiven Operator Characteristic (ROC) analysis was used (MCNEIL et al 1975; O"DONNELL et al 1980). In this method a range of arbitrarily chosen thresholds is used in the assigment of an object to one of the two classes. This will result in a range of corresponding specificity and sensitivity values which can be plotted as a ROC curve. That threshold value which resulted in a specificity and a sensitivity both 
nearest to 100 percent was considered the optimal one for the assignment of an object (leg) to one of the two (angiographic) classes.

In order to include already beforehand only the most discriminating parameters in the parameter selection procedures, a two-sided significance limit of $p<0.001$ was employed in ISPAHAN. If occasionally mean values or proportions are compared with other intentions than the selection of discriminators (e.g. to indicate a trend or support a hypothesis), the more lenient significance criterion of $p<0.05$ will be used in this study. In the latter case the $p$-value will allways specially be mentioned.

The test results in the present study could be either scores with a discrete probability distribution (pulsation scores, types of response of the ankle pressure to exercise and qualitative Doppler signal scores) or values of parameters with a continuous frequency distribution (pressures, qualitative signal parameters and angiographic scores).

It is realized that it is not allowed to calculate a Standard Deviation (SD) of data with a non-continuous frequency distribution. However, in following chapters the term "Standard Deviation" ("SD") will occasionally be used in the comparison of results of scores of the discrete type. In that case the term should be understood as the "square root of the sum of the squared deviations from the mean" of those scores. 


\section{RESULTS}

In the first part of this chapter (10.1) a survey is given of the results of the warious tests either by angiographic class or by inflow score of the angiogram and the findings are discussed. In addition some results are compared to each other by patients" symptoms if a relation between the results and the symptoms is likely. The selection of test parameters for further statistical analyses will be discussed in the second part of the chapter (10.2) and the chapter will be completed by a discussion on the diagnostic value of the different parameters (10.3).

Tables presenting detailed information about the results obtained are presented in an appendix (A-Tables). Part of these results will also be presented in figures, inserted into the text, for easier reference.

\subsection{SURVEY OF THE RESULTS AND DISCUSSION}

\subsubsection{Angiograms}

If in this and the following sections the term "angiogram" is used reference is made to the angiographic view of the arteries belonging to one leg (section 9.7.1). Of all patients two such angiograms - one for each side - were available for analysis. Each angiogram was characterized by two figures: a score for the proximal or inflow section and a score for the distal or outflow section, as described in section 9.7.1.

A reliable determination of the angioscore was not considered possible by the reviewers in 15 inflow and 14 outflow sections. The various combinations of inflow and outflow scores found in the 192 patients' angiograms in this study are given in Table A-1. The angiograms were classified according to the classification system described in section 9.7 .1 and Figure 9.4. The mean values $( \pm$ one SD) of the inflow and outflow scores of the angioclasses 1 through 4 are given in Table A-2; the same data are presented diagrammatically in Figure 10.1 .

These classes appear to be very well distinguishable from each other with regard to their mean inflow and outllow score. It also seems justified to consider

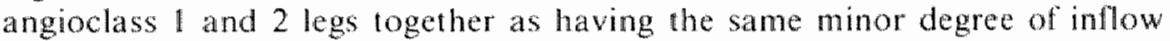
obstruction versus a group of legs with a significant inflow obstruction of equal severity consisting of the angioclass 3 and 4 limbs. Neither did the mean inllow scores of class 1 and of class 2 legs differ significantly from each other, not were the mean inflow scores of angioclass 3 and 4 legs significantly different from each other $(p>0.05)$. There was a difference between the mean inflow scores of both the class 1 and 2 legs on the one hand and the means of these scores of class 3 and 4 limbs on the other hand $(p<0.001)$. In a similar way the mean outhow scores of class 1 and class 3 legs were not different from each other $(p>0.05)$, whereas the difference between the mean outflow scores of class 2 and class 4 legs reached some significance $(0.005<\mathrm{p}<0.01)$. A highly significant difference existed between the mean outflow scores of both the class 1 and 3 limbs on the one hand and both the class 2 and 4 legs on the other hand $(p<0.001)$. It, therefore, seems to be justified to further use this classification in correlative studies. 


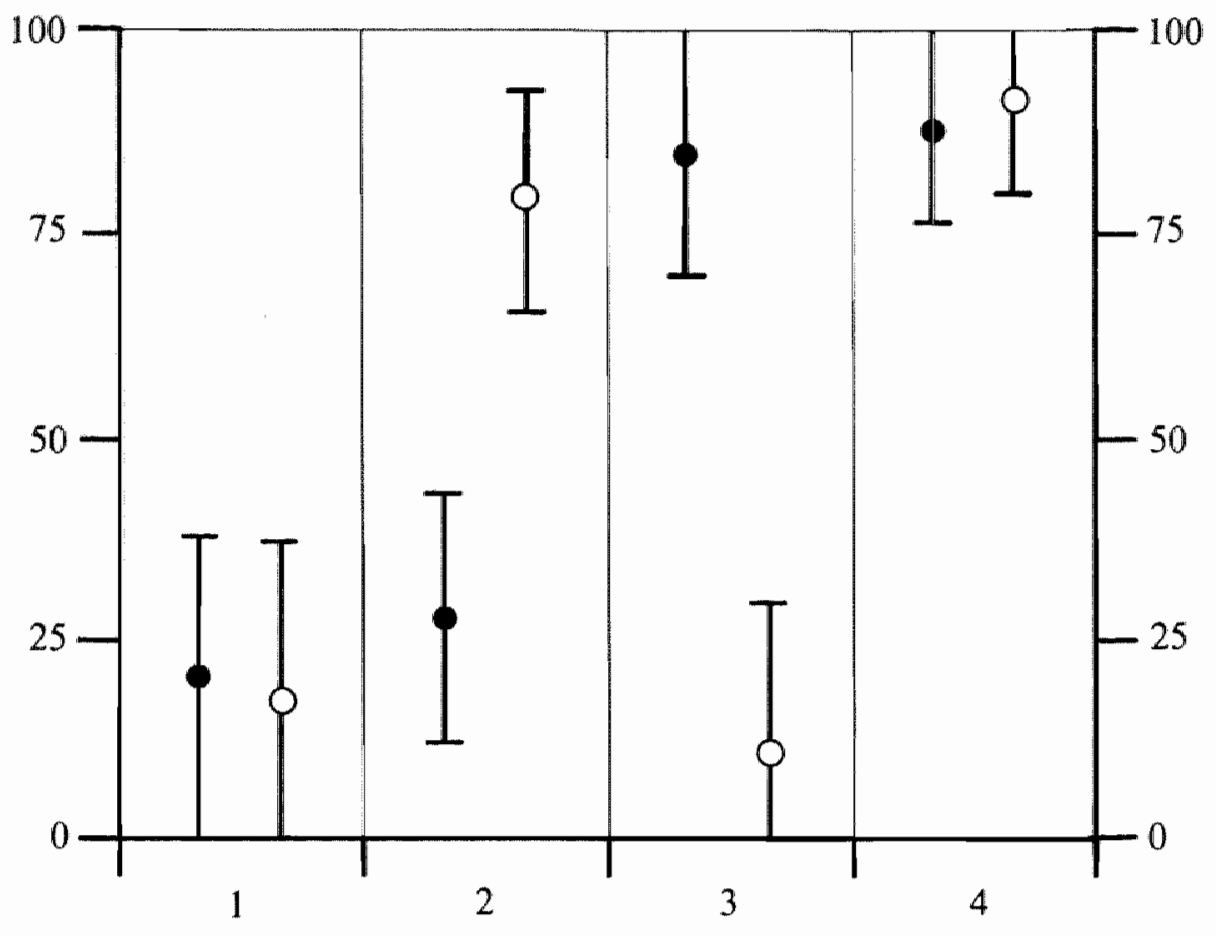

ANGIOGRAPHIC CLASS

FIGURE 10.1

Angiographic classes of patients'legs with the mean values $( \pm S D)$, of their angiographic inflow and outflow scores.

In 42 angiograms ( 21.8 percent) aneurysmatic segments were found in the inflow section. In 81 percent of the cases they were localized in the aorta. The high number of aneurysmatic angiograms can be partly explained by the fact that each aorta contributes to two angiograms. In 70 percent of the angiograms with an aneurysmatic aorta also more distal inflow segments were seen with aneurysmatic disease. The aneurysms were evenly distributed over all angiographic classes of patients' legs.

The relative occurrence of the various angiographic classes within each of the four patient groups (a-symptomatic, claudication, rest pain, necrosis) is summarized in Table A-3. In the proportional occurrence of the various angioclasses within the different patients' groups some trends may be noticed. Angioclass 1, standing for the absence of serious obstructive lesions, was relatively frequently seen in the a-symptomatic patients. The rest pain patients often had anglographic evidence of multilevel obstructive disease (angioclass 4). 


\subsubsection{Clinical examination}

The clinical data considered in this study were the pulsation scores and the results of the auscultation of the arteries for bruits.

For all patients' legs with a classifiable angiogram and for all normal legs, the scores of the pulsations of the common femoral artery, of the popliteal artery and at the ankle were compared with the angioclass of the leg. As "ankle pulsation" score the highest of the two scores - posterior tibial artery or dorsalis pedis artery - was selected for each leg. Usually (78 percent) both scores were equal; if not so, the posterior tibial artery most frequenty had the higher score. Details of pulsation scores at the groin, the knee and the ankle by angioclass are given in Table A-4. A more clear picture of the relationship between the angioclassification and the pulsation scores arises if the data are presented in a proportional manner (Figure 10.2). Theoretically, important obstructions are

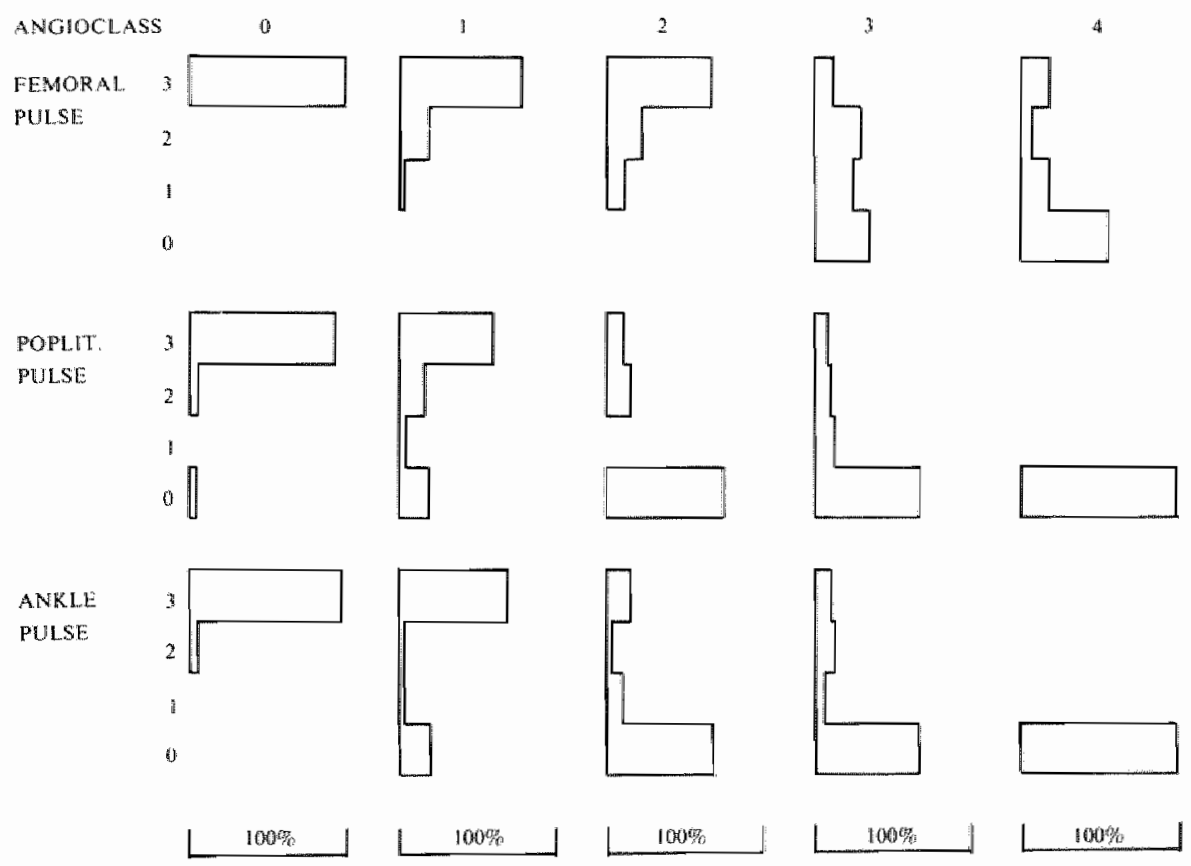

FIGURE 10.2

Proportional occurrence of the pulsation scores at three levels by angiographic classification of the limb.

not likely to be found on the angiogram if the pulsations at all three levels are strong. If an important outflow obstruction exists in the absence of serious inflow lesions, the femoral pulse may be expected to be strong and the more distal pulses weak. In cases of a proximal obstruction all pulses will be diminished to some degree, whereas in obstructions at two levels the latter phenomenon will be more pronounced. The results presented in Figure 10.2 support these considerations, which are the basis of the thinking process of the clinician when he tries to locate the site of arterial obstruction in the leg by pulse palpation. The legs with a class 1 angiogram had a good proportion of strong 
pulsations at all three levels. In the legs of angioclass 2 the femoral pulses were nearly all strong whereas the popliteal and ankle pulses were not palpable in the majority of the cases. In angioclass 3 legs the majority of the three pulses was weak or absent. In the legs scoring an angioclassification 4 , the knee and ankle pulsations were always absent.

In the evaluation of aortoiliac obstruction the femoral pulse strength and the presence of pelvic bruits are often considered to be important signs by the clinician. Their value was discussed in section 2.2 . The correlation between the femoral pulse strength and pelvic bruits on the one hand and aortoiliac obstruction on the other hand was examined in the patients" material of this study. If either at rest or after the walking test a bruit was heard over the aortic bifurcation or the corresponding pelvic or common femoral region, a leg was considered to have a pelvic bruit. In 134 of all patients" legs $(69.8$ percent $)$ such a bruit was present; in 43 of these legs ( 22.4 percent) the bruit was only heard after the walking test and not at rest. In none of the normals a bruit was found at rest and in one normal only soft bruits were heard over both femoral arteries after the treadmill test. Eight combinations are possible of the different femoral pulsation scores $(0,1,2,3)$ with presence $(+)$ of absence $(-)$ of a bruit. In Table A-5 the corresponding mean inflow scores ( \pm one SD) for the various pulsation/bruit combinations are given. If the pulsation/bruit sicores were ranked according to the increase of the corresponding inflow score, the sequence was $3-, 2-, 3+, 2+, 1+, 1-, 0-, 0+$. There existed a clear trend towards higher inflow scores in legs with weaker femoral pulsations. However, between none of the pairs of neighbouring pulsation/bruit combinations from the mentioned sequence a significant difference (criterion $p<0.001$ ) in the mean value of the inflow score was found. For this reason this detailed subdivision of clinical parameters seems not very useful, the more so since the grading of the pulsations as well as the detection of bruits are rather unreliable (section 2.2.1). Therefore, the relationship between the femoral pulse strength, disregarding bruits, on the one hand and the inflow score on the other hand was looked at (see also Table A-5). The mean inflow scores of the legs grouped by femoral pulse strength all differed significantly from each other $(p<0.001)$. The correlation between the femoral pulse strength and the inflow score of the legs, shown in Figure 10.3, appeared to be a significant and a rather good one $(r=-0.78, p<$ $0.001)$.

\subsubsection{Systolic blood pressures in the limb at rest}

The mean values of the blood pressure indices at the thigh and at the ankle were determined for the normal legs and for the patients' legs, grouped according to the main complaint of the patient. These means were also calculated separately for the symptomatic legs of the patients since it could be expected to find lower values for the symptomatic than for the a-symptomatic legs. The mean walues for the pressures at rest (土 one SD) versus the symptoms are given in Table A-6.

A correlation between the patients' symptoms and the thigh pressure index may not be expected and it has not been reported in literature (see section 5.4). In the material of this study significant differences were not found between any of the mean values of the thigh pressure indices when arranged by symptom (all $p>0.01)$. Only the means of normals and of the legs of a-symptomatic patients which themselves did not differ significantly from each other, $(p>0.4)-$ differed significantly $(\mathrm{p}<0.001)$ from the mean values of the claudication 


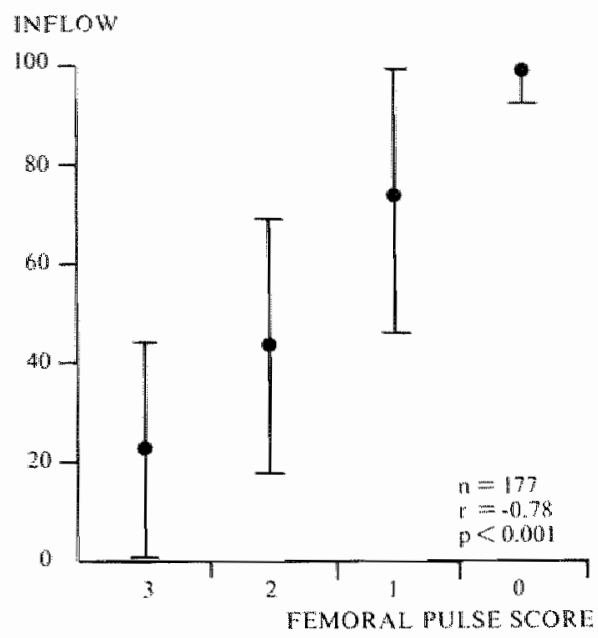

FIGURE 10.3

Correlation between the femorat pulse strength (score) and the angiographic innow score.

Dots represent the mean value of line inflow score, the bars I $1 \mathrm{SD}$.

patients and the rest pain patients, but not from the vallue of the necrosis group. It made no difference in significance whether both legs or only the symptomatic legs were compared.

The same was found for the ankle pressure indices, namely no significant difference between the values of the claudicants, the rest pain and the necrosis patients (criterium for significance $p<0.001$ ). Only the mean values of the normals and the a-symptomatic patients - which themselves did not differ significantly from each other - were different from the values of the legs of claudication patients and rest pain patients. Even if more lenient criteria for significance were applied ( $p<0.01$ after YAO $(1970,1973)$, see also section 5.4), no difference could be found. Consequently, the data in this study showed the trend of lower ankle pressure indices in the more severe clinical stages of the disease, as mentioned in section 5.4 , but no significant differences between these stages. The finding of the lowest ankle pressure indices in patients with ischemic foot lesions, as discussed in the literature review section 5.4 , could not be confirmed in this study. The necrosis patients had a mean ankle pressure index which did not differ significantly $(p>0.01)$ from the value of the other patients and even not from the normals. Probably the "necrosis patients" in this study are different from the "(impending) gangrene" patients seen by others (section 5.4) and they do not fit to the usual clinical grading system of progressive ischemia. Of the six "necrosis" patients only one had an ischemic aspect of the foot with extensive tissue necrosis and that patient had an ankle pressure index at rest of 0.25 . The other five only suffered from very localized necrosis of one toe. They otherwise had a normal aspect of the foot and palpable pulses making an emboligenic distal occlusion the most probable reason for the necrosis. This was the more likely since half the number of angiograms of the necrosis patients was without gross obstructive lesions (see Table A-3) and the ankle pressure indices of all these patients but the mentioned one were nearly or completely normal.

The average thigh and ankle pressure indices for the legs belonging to the various angiographic classes are given in Table A-7 and diagrammatically presented in Figure 10.4.

The mean thigh pressure index of legs in which either the inflow or the 

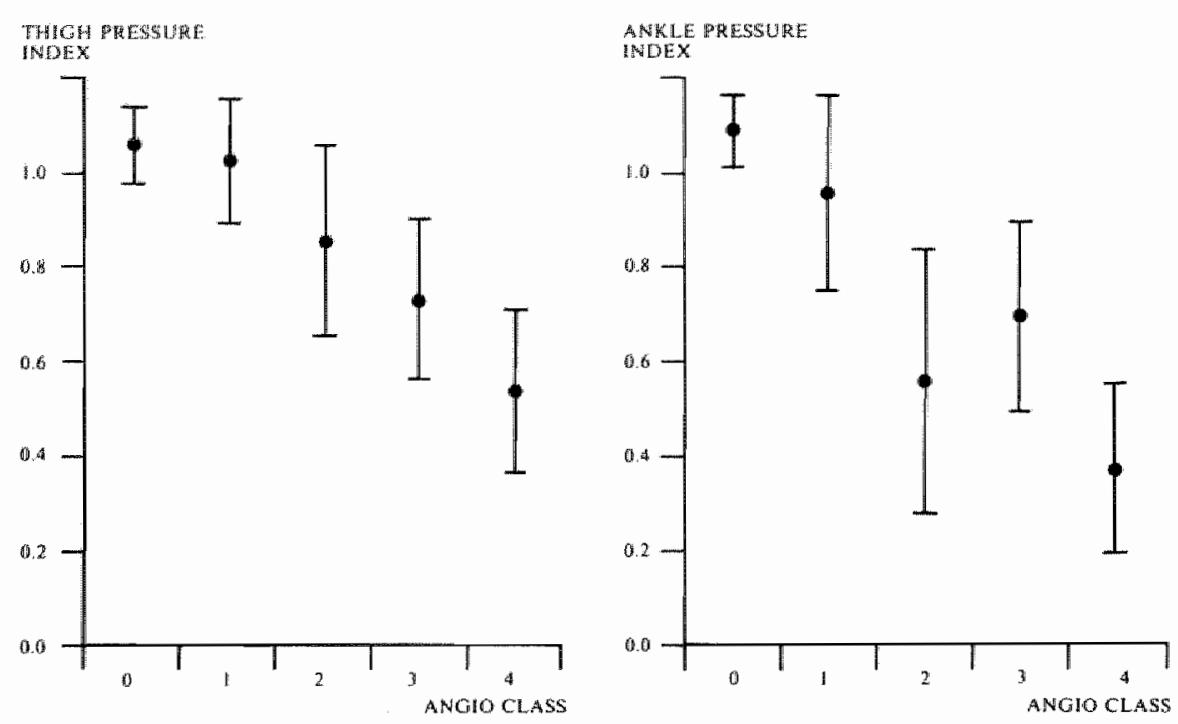

FIGURE 10.4

Mean values of the thigh pressure index and of the ankle pressure index of llegs of different angiographic classes.

Dots represent the mean value of the indices, the bars $\pm 1 \mathrm{SD}$.

outflow score or both were above 50 was significantly lower than the mean thigh pressure index of the normal legs (class 0 ) or the legs without important obstructions (class 1). This reflects the fact, know from other studies (section 5.5.3.), that on the average the thigh pressure index is lowered by obstructions proximal to the groin (class 3 ) as well as obstructions localized mainly distal to the groin (class 2). The (reduced) mean thigh pressure index of angioclass 2 legs was not significantly different from that of angioclass 3 legs ( $p>0.025$ ). A diminished thigh pressure index, therefore, is no specific indication of inflow obstruction. The same conclusion had already been drawn by others as discussed in the sections 5.5 .4 and 8.1.

The mean of the ankle pressure index of the normal control legs was significantly higher than the mean values of the legs in all the patients' angioclasses $(p<0.001)$. Also class I legs had a significantly higher mean ankle pressure index than the legs grouped in class 2,3 or $4(\mathrm{p}<0.001)$. No significant difference was observed between the mean ankle pressure index of the legs with angiographic obstructions in the inflow section (class 3) and of the legs with obstructions in their outflow section (class 2$)(\mathrm{p}>0.05)$. This is in agreement with other reported studies in which ankle pressure measurements at rest did not permit a differentiation between obstructions in the aortoiliac and the femoropopliteal pathway (see section 5.5.4). In this study the difference between the mean ankle pressure index of angioclass 2 legs and of angioclass 4 legs was not significant $(\mathrm{p}>0.025)$.

Despite the fact that the thigh pressure index is a measure of inflow obstruction of low specificity, in the own material a good correlation was found between the angiographic inflow score and this pressure index $(n=177, r=$ 
$-0.79, p<0.001)$. This is illustrated in the scatterdiagram of Figure 10.5. The figure also illustrates how obstructions in the arteries distal to the groin cause a lowering of the thigh pressure index: the legs with an outflow score above 50 (open circles in Figure 10.5) are mainly found in the lower part of the scatterdiagram. The correlation between the inflow score and the thigh pressure index, however, is hardly influenced by the presence of these legs. For, if only the legs were considered of which the outflow score is below 50 , nearly the same correlationcoefficient was found $(n=134, r=-0.80, p<0.001)$. The same applied to the correlation between the inflow score and the thigh pressure index. of the legs with a high outflow score only $(n=35, r=-0.71, p<0.001)$.

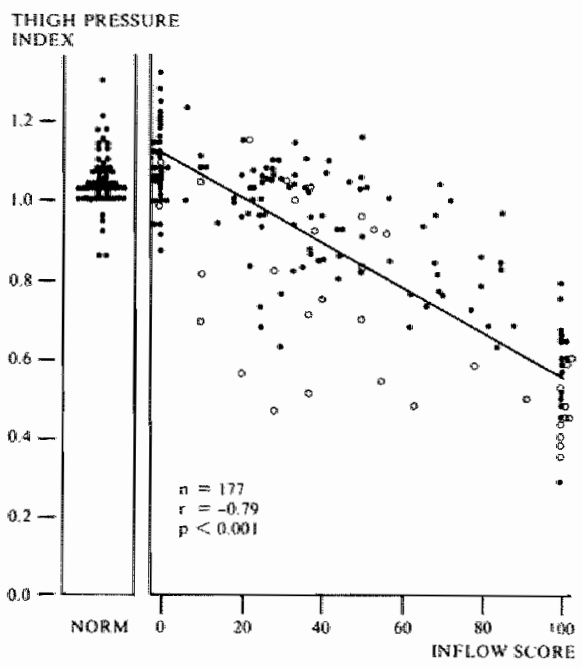

FIGURE 10.5

Correlation between inflow scores and thigh pressure indices in patients'legs.

The left column gives the thigh pressure indices of the healthy test persons.

(Dots represent the legs without significant outflow obstruction (owtflow score $\leqslant 50$ ). the circles the legs with significant outflow obstruction (outflow score $>50$ )).

10.1.4. Walking test

All normals completed a full 5 minutes walk on the treadmill without any complaint. Two patients were excluded from the test because of angiographically documented, severe coronary artery disease. The performance of the other 94 patients is summarized in Table A-8. Four patients had to stop the test within 5 minutes for complaints other than claudication. One experienced "dizziness" and abruptly stopped walking after 45 seconds, the other three developed severe dyspnea without angina pectoris within 2-3 minutes. Of the asymptomatic patients all but one completed the full 5 minutes on the treadmill. Of the 61 claudication patients 9 (14.5 percent) walked the full 5 minutes, although 4 of them developed typical claudication before that time. Remarkably, 3 of the 6 patients with necrotic lesions of the foot walked the full 5 minutes. This is another indication that the "necrosis" patients in this study did not belong to the most severe category of ischemia, as in most other series, but rather were suffering from embolic complications in only moderate obstructive arterial disease (see also section 10.1.3). The one patient with "rest pain" who yet walked for five minutes (Table A-8) must have belonged to this same category; that patient had a continuously painful bluish hallux (hence her classification in the rest pain group) probably caused by an embolus from a minor irregularity in the popliteal artery and an otherwise angiographically normal arterial system. 
For those who developed real claudication on the treadmill the average time of onset of claudication (claudication time) and the average time at which the test was stopped (maximum walking time) - expressed in seconds from the start of the test - are given by patients' category in Table A-9. On an average the claudication patients developed their complaints later than the rest pain patients and they had a longer maximum walking time. The differences, however, were not significant, neit her for the claudication time $(p>0.2)$ nor for the maximum walking time ( $p>0.025$ ). In 52 percent of the patients there was less than 100 meters difference between the pain free distance as indicated before the test by the patient and the distance actually walked on the treadmill. Only 8.5 percent had overestimated their capacity by 100 meters or more and the other 40.5 percent performed better on the treadmill than they would have thought of before. The reasons for the latter may include such factors as an inability to judge distances, a difference between treadmill walking and an unsupported gait and the wish to satisfy the examiner.

The inconclusive results of the performances during the walking test make it impossible to use the results of it for an objective grading of the functional impairment of the vascular patient. The author, however, got the impression that the test was a great help in a better judgment of the disability of the patient. Similar opinions are found in leading textbooks on diagnostic techniques in vascular disease (STRANDNESS 1978).

\subsubsection{Ankle pressure response to exercise}

For all normals and for those patients who performed a walking test, the ankle pressure response data were classified as described in section 9.7.3. A total of 240 response curves could be analyzed. In the further analysis it will be assumed that - according to what has been discussed in section 5.2. - the profoundness and the duration of the post-exercise ankle pressure fall reflect the functional severity of the obstructions in the arteries of the leg. However, the type of response curve is not only related to the vascular status of the leg but also to the workload during exercise (STRANDNESS 1969, pp. 61-91). In this study the workload was not standardized but depended on such variables as the maximum walking distance, the way the person walked and his body built. Also the fact that patients with unilateral claudication usually only stress the symptomatic leg to its maximum and not the other leg, caused variations even between the two legs of one person. Yet, it was considered worthwhile to compare the ankle pressure responses after these non-uniform workloads, since many noninvasive vascular laboratories employ a similar walking test. Various studies have been reported in which the ankle pressure response after a treadmill test, which was stopped whenever the patient experienced severe claudication or after 5 minutes if no claudication occutred, was of diagnostic value (see sections $5.5 .1,5.5 .2$ and 5.5 .4 ).

In Table A-10 the number of the various response types within each category of tested persons is given. For the symptomatic patients also the responses of the leg with the more severe complaint (or the symptomatic leg) are presented separately in that table. In some claudication patients who had bilateral complaints of equal severity both legs were counted as symptomatic. The more severely diseased patients responded to exercise with a high proportion of pathological pressure falls, whereas the a-symptomatic patients had mainly normal responses. This trend was also reflected in the mean value of the 
response for the various categories of tested persons (Table A-10). None of these values for the symptomatic patients were significantly different from each other ( $p$ always $>0.01)$. The difference between the mean score of the legs of normals and a-symptomatic patients on the one hand and the mean scores of both the two legs and/or only the a-symptomatic legs of the claudication or rest pain patients on the other hand was statistically highly significant $(\mathrm{p}<0.001)$. This might make the described ankle pressure response classification useful for the differentiation between normals and vascular patients but hardly for grading the severity of the disease.

The relationship between the angiographic classification and the ankle pressure response type of all legs with a classifiable angiogram is given in Table A-11. To facilitate their comparison these same data are also presented diagrammatically in a proportional way in Figure 10.6. The average value of the

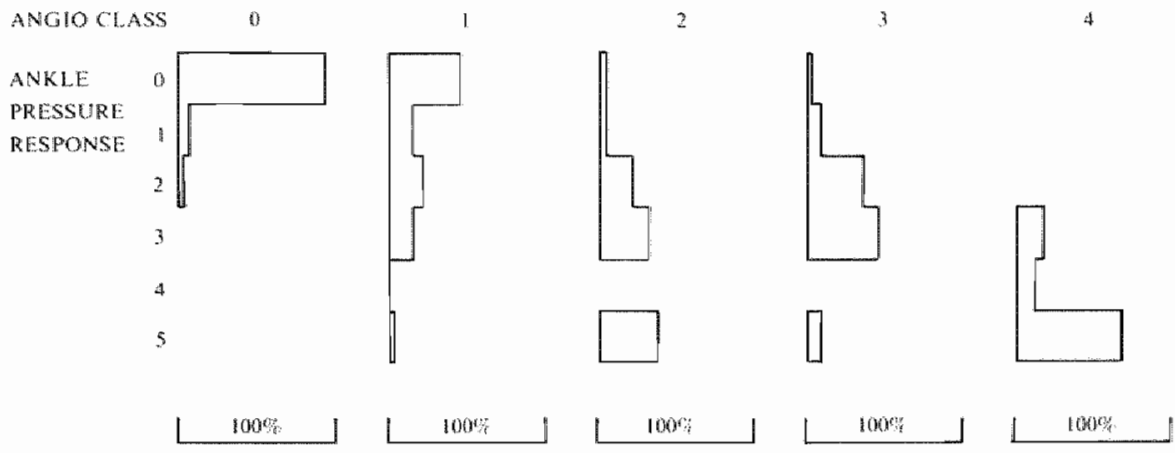

FIGURE $\| 0.6$

Proportional occurrence of the different ankle pressure response types by angiographic classification of the limbs.

response score was not significantly different between angioclass 2 and angioclass 3 legs $(p>0.05)$. Between angioclass 2 and angioclass 4 legs the mean response score value was different at a reasonably high significance level $(0.001$ $<\mathrm{p}<0.005$ ). All other mean response score values were different from each other with a high degree of significance $(p<0.001)$. This is in agreement with reports of others who found a correlation between the type of ankle pressure response and the presence or absence of angiographic obstruction (section 5.5 .1 ), the presence of either single level or multilevel disease (section 5.5.2) but overall no difference between the type of response curve in cases of proximal obstruction (like in angioclass 2 legs) and the responses in distal obstructive disease (like in angioclass 3 legs) (section 5.5.4.).

\subsubsection{Aorto-femoral transit time measurement}

A sonagram on which both the aortic and the femoral systolic upslope could be clearly distinguished was a prerequisite for a reliable determination of the aortofemoral pulse wave transit time (TT). If one or both signals could not be recorded or got "submerged" in artifacts the TT could not be calculated. This was the case in a total of 60 legs ( 24.2 percent). Nine times the femoral signal was too weak for recording, 3 times faulty recording techniques - only discovered 
during replay - were the cause and in the other 48 cases no aortic signal was obtained or only one of poor quality. If for any reason the aorta was inaccessible to Doppler investigation, the TT for both aortoiliac pathways could not be determined. The main reasons for this were severe obesity ( 6 times), extensive scars of the abdomen ( 2 times) or continuous bowel movements ( 4 times). In 7 patients only turbulent, unusable aortic signals were obtained, although all circumstances for good recording seemed present (thin patient, well palpable aorta, no scars, no bowel artifacts). Three of these patients had abdominal aneurysms. In 10 persons a recording was usable on one side but it was disturbed on the other side due to mean while interfering bowel mowements. Disregarding the three cases of faulty recording technique, the TT could not be determined due to factors related to the tested person in 23 percent of the cases. In 19.4 percent the problem was in obtaining and aortic signal. Also others have experienced these problems in recording a satisfactory aortic signal. The group of Gosling which did much work on aortic analysis, reported 5 failures to obtain an aortic signal in 33 patients (15 percent failure) (GOSLING and KING 1978).

The mean walues of the observed T'T's and of the normalized TT's (for definition see sections 7.2 and 9.7.4) for the legs within the various angioclasses are summarized in Table $\mathrm{A}-12$. The observed TT's were considerably overlapping. The lowest values were found in angioclass 1 and 2 legs. These mean values - which did not differ significantly from each other, $p>0.5$ - were significantly different from the mean values of the normals (class 0 ) and of the legs with an inflow obstruction (class 3$)(p<0.001)$. The mean value of the normals was not significantly different from that of the legs with an inflow obstruction (class 3 ) ( $p$ $>0.3$ ). The small number of class 4 legs, available for analysis, made statistical manipulations of the results of that group senseless. The explanation for these findings might be that patients without important obstructions nevertheless have stiffer arteries than the normals and hence shorter pulse wave TT's (section 7.1 and 7.2). In case of atherosclerotic inflow obstructions, among which total occlusions, the mean TT"s are increased compared to the values of unobstructed aortoiliac pathways and may reach normal levels.

Normalication of the TT's (Table A-12) did not give a better separation between the angioclasses. For all combinations of angioclasses the mean values were not significantly different from each other $(\mathrm{p}>0.025)$. The average of the normalized TT"s for the normal testpersons might be expected to be about 100 percent. In this study a value of 150 percent was found. A difference in recording and calculation techniques for 'T"T determination between the one employed by Gosling's group and the one used in this study is unlikely as a cause for this descrepancy. During personal visits to Gosling's laboratory at Guy's Hospital, London, the author could ascertain the similarity of the methods. Possibly the explanation must be sought in the fact that the normal values for the iliac segment were extrapolated from Goslings' findings in the femoropopliteal pathway (as described in section 9.7.4). It is imagineable that between these two vascular pathways differences in compliance exist which might be responsible for the discrepancy between the expected and the calculated normalized TT's in normal persons in the present study.

In Figure 10.7 the relationship between the inflow score of the legs and the aorto-femoral. TT is presented in a scatterdiagram both for the observed and for the normalized TT's. Only a very low and not significant correlation was found. The inclinations of the drawn regression lines in the scatterdiagrams of Figure 10.7 suggest that the TT increases with a higher angiographic inflowscore. The 
inclination, however, is caused by the inclusion of completely blocked inflow segments (inflow score $=100$ ) with very long TT's in this analysis as can be demonstrated by exclusion of these legs from analysis. The regressioncoefficient then approach to zero as shown by the course of the dotted regression lines in Figure 10.7 .
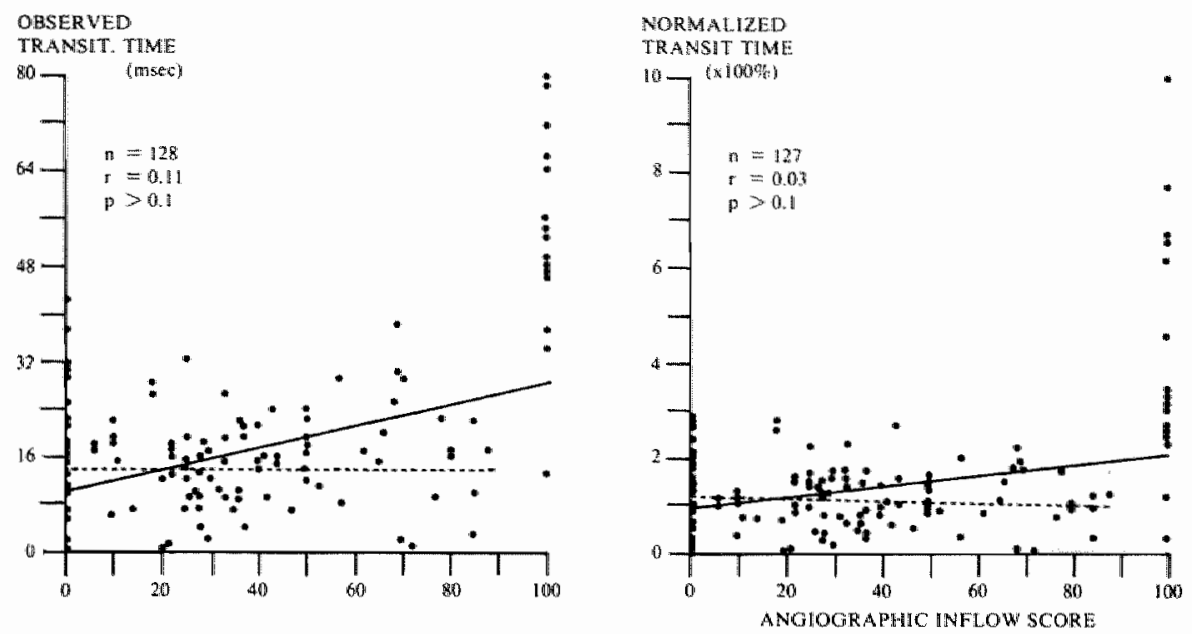

FIGURE 10.7

Correlations between the observed and the normalized transit time values and the angiographic inflow scores of the legs.

The drawn regression lines apply to the whole group of legs, the interrupted regression lines to the legs exclusive of the ones with an outflow score of 100 .

\subsubsection{Doppler signals}

\subsubsection{Characteristics and quality of the Doppler sonagrams}

In obtaining sonagrams from the three anatomic sites (aorta, femoral artery, ankle/pedal arteries) serious problems were encountered only with the signals of the abdominal aorta. In section 10.1.6 some of these difficulties were already discussed. In the full analysis of the directional aortic signals the problems were even greater. Sometimes the systolic forward deflection of the sugnals was well recognizable on a sonagram - enabling the calculation of a transit time - while at the same time the other signal components (especially the reverse ones) could not be delineated. In such cases neither a qualitative nor a quantitative signal analysis was possible. A second problem was the incertitude about the aortic origin of even good abdominal signals. It has been reported that Doppler signals from the inferior mesenteric artery can be mistaken for aortic signals (GOSLING and KING 1978). This could be suspected if on the one hand the abdominal signal either represents a continuous systolic-diastolic flow or is monophasic in character whereas on the other hand the femoral signal contains forward and reverse components (GOSLING 1978, personal communication). In cases of a pathological femoral signal this criterion cannot be used. During the period of data collection the author was not yet aware of this possible source of 
misinterpretation of the "aortic" signal. A part from the above mentioned one no reference could be found about this subject.

Retrospectively 31 aortic signals ( 25 percent of the total) had to be rejected for analysis because of poor quality. With one exception they were all of patients. The acceptable quality of all other aortic signals, both of patients and of normals, make technical factors less likely as the cause of the poor quality of these 31 signals. A review of the angiograms of the 30 patients whose aortic Doppler signals precluded further analysis showed the following: 10 had an abdominal aneurysm ( 33.3 percent), 2 had undergone prosthetic replacement of the aorta ( 6.7 percent), 2 had a high degree of stenosis at the aimed at site of recording in the aorta, whereas in the remaining 16 (53.3 percent) moderate stenotic lesions were seen on the angiogram. In normals the quality of the aortic signal, if obtainable, was good. In the light of these findings the failure to record a clearly delineated aortic signal may fasten the suspicion on pathology of the aorta. In 13 patients and 4 normals (13.7 percent of the total) no aortic signals could be recorded at all due to obesity or disturbing bowel sounds. Thus, no Doppler signal parameters could be obtained in a total of 48 aortas in this study (38.7 percent). In the patients population this was even the case in 44.8 percent. For that reason the results of the analysis of the aortic signals of the remaining persons will not be discussed since they would heardly allow any firm conclusions. Consequently, also the calculation of a "Damping factor" of the aortoiliac pathway (section 7.3 ) became impossible.

Representative normal sonagrams of aorta, common femoral artery and an ankle artery are shown in Figure 10.8. The influence of obstruction in the arteries and/or the induction of hyperemia on the sonagraphic patterns is demonstrated in Figure 10.9.

Regularly sonagraphic patterns were found which contained artifacts or which indicated flow disturbances at the recording site. Some of these patterns are shown in Figure 10.10. These patterns may provide valuable diagnostic information. For instance Figure 10.10d shows a strong forward and reverse signal simultaneously occurring during systole as an indication of turbulent flow due to the presence of a nearby stenosis upstream (GOSLING and KING 1978; BLACKSHEAR et al 1979). Venous contamination of the arterial signal (Figure 10.10a) is readily recognized and can usually be prevented during recording. The "spikes" in the reverse signal coinciding with the beginning of

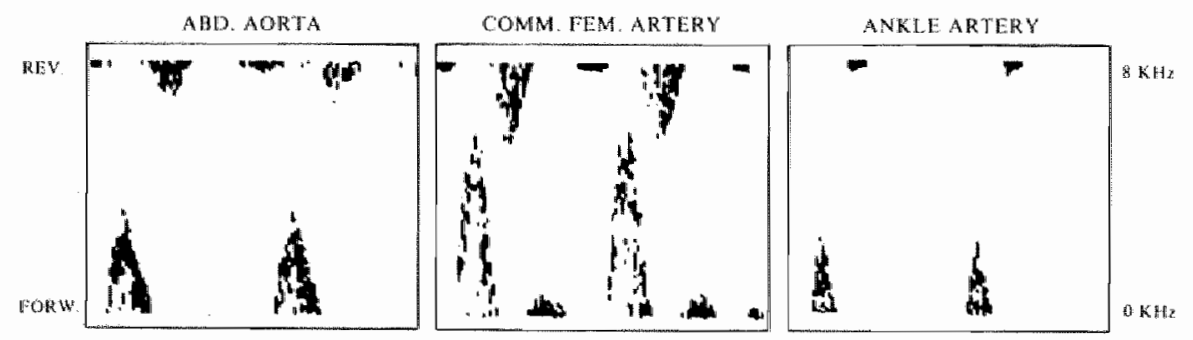

FIOURE 10.8

Representative normal sonagrams of the abdominal aorta, the common femoral artery and one of the arteries at the ankle.

For explanation of sonagrams see legends to Figure 4.4. 

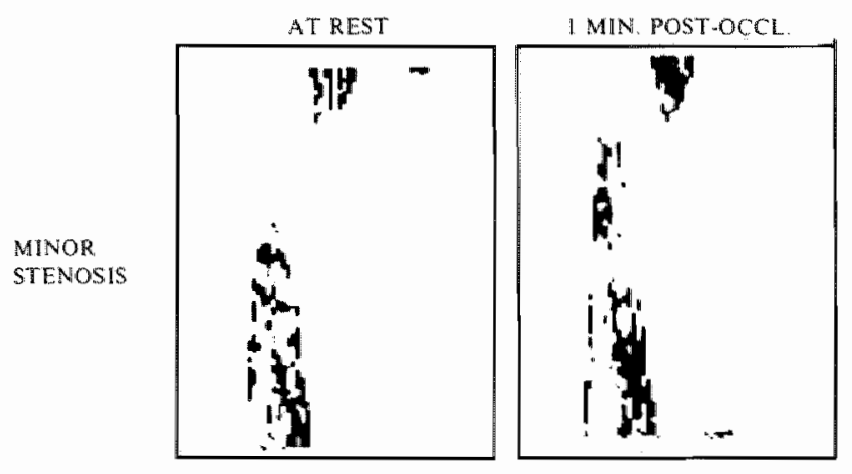

MHN.POST WOCLL
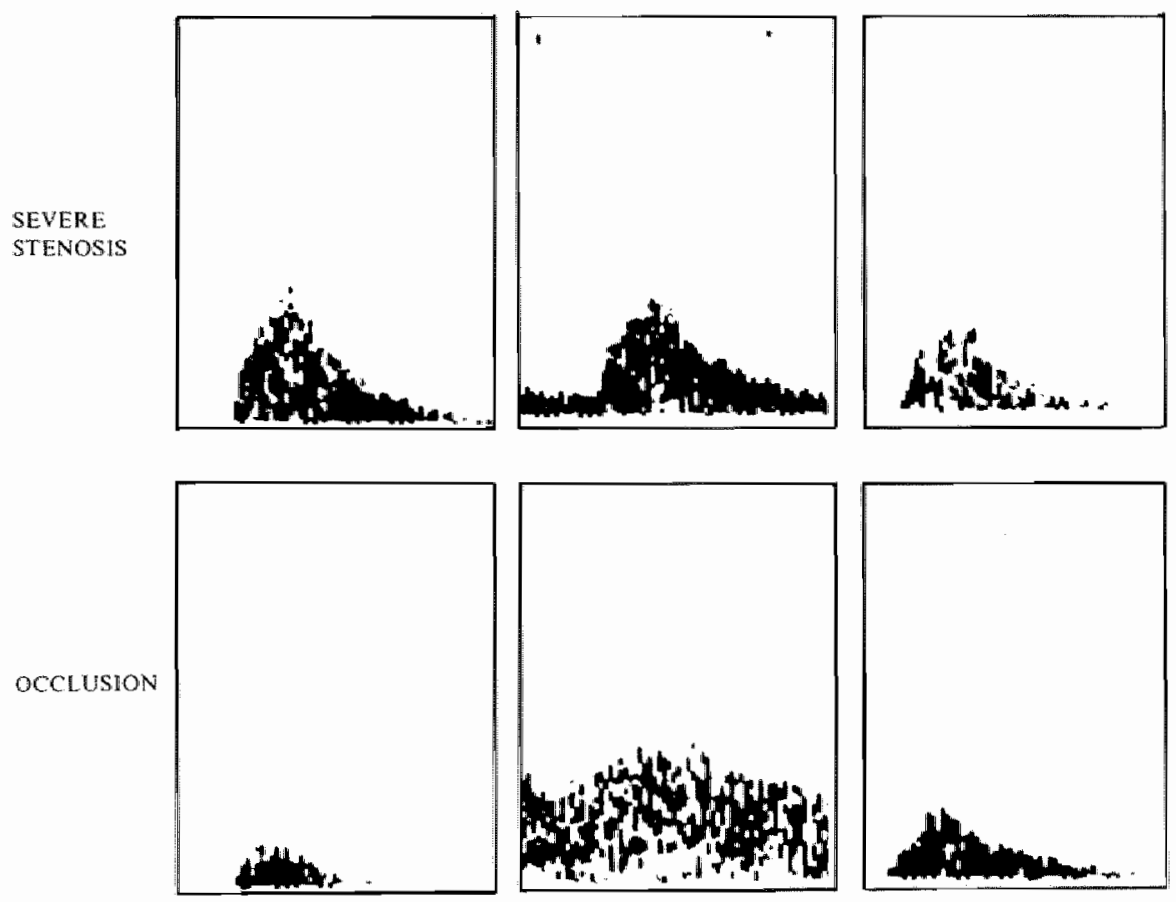

\section{FIGURE 10.9}

The influence of proximal obstruction and/or hyperemia on sonagraphic Doppler signal patterns.

Femoral sonagrams of legs with isolated minor stenosis (upper row), severe stenosis (middle row) and occlusion (bottom row) of the iliac artery. Sonagrams are given for conditions at rest (left column), I minute (middle column) and 3 minutes (fight column) after release of thigh occlusion. (For explanation of sonagrams see legends to Figure 4.4). 

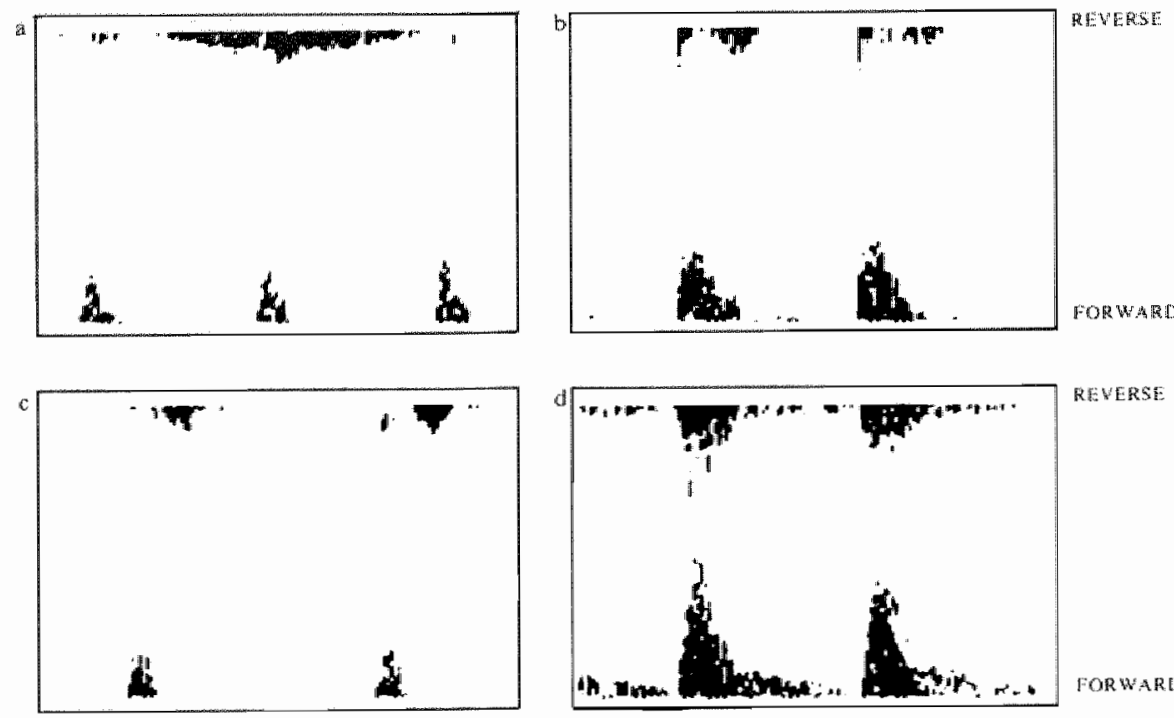

REWERE:

EFIGURE 10.10

Sonagrams displaying artifacts or flow disturbance.

a. Simultaneous display of arterial and venous (top) signal. b. Spikes in the reverse signal coinciding with the beginning of the systolic forward signal. c. Mirror images of the forward signal in the reverse signal. d. Simultaneous strong forward and reverse signal due to turbulence of llow.

For explanation of sonagrams see legends to Figure 4.4.

the systolic forward upslope (Figure 10,10b) occurred only infrequently and no good explanation for them could be found. They possibly are a result of vessel wall movements but they may be due to instrumental factors as well. Low intensity mirror images of a forward signal visible on the reverse trace (Figure $10.10 \mathrm{c})$ are possibly caused by the simultaneous insonation of several parts of a curving vessel or of a main vessel and its side branch (GOSLING and KUNG 1978). Also the possibility of cross-talk in the recording equipment and/or inadequate channel separation could not be excluded in these cases (section 9.4.3.).

Patterns like the ones just discussed and shown in Figure 10.10 raise the question on whether certain parts of the sonagram should be considered artifacts and, therefore, ought to be excluded from outlining when performing analysis of their maximum frequency envelopes. This was proposed by GOSLING and KING (1978). By doing so too liberally one takes the risk of considering certain patterns artifacts just because one cannot reasonably cxplain them yet or because they were not described in other studies. In that way important (patho)physiological information contained within the original sonagram might be concealed. In view of possible future computerized pattern analysis techniques - in which artifacts most likely will only be distinguished from real signal contents with difficulty - it neither seems appropriate to trace a smooth waveform envelope through the sonagram and leave out certain parts as artifacts too easily. For the purpose of this study the following rules were followed while digitizing the sonagram. Apparent venous contamination of the 
arterial signals (Figure 10.10a) was excluded from outlining and the same applied to the spikes (Figure $10.10 \mathrm{~b}$ ) and the faint arterial mirror images (Figure $10.10 \mathrm{c}$ ). However, the strong reverse components of a signal characteristic of turbulence (Figure 10.10d) were outlined. They fully contributed to the computer-calculated signal parameters. The specific problems caused by doing so will be discussed in the next sections. In the qualitative, at sight, scoring of the sonagram, reverse flow - prerequisite for a score 3 - was only counted as such when not occuring simultaneously with the systolic forward signal.

\subsubsection{Qualitative Doppler scores}

The qualitative classification (section 9.7.5.1) of the femoral and ankle sonagrams recorded at rest apeared to be a simple procedure usually performed at a glance. The results were analyzed along the same lines which werefollowed for the pulsation scores (section 10.1.2). The arterial pulsation and the character of the velocity pulse, as recorded with a Doppler meter, both behave in a quite similar way in case of arterial obstruction. Distal to an obstruction they become less pulsatile and thus lower in score. On the analogy of what was described for the palpable pulse strength in section 10.1.2 it could be expected that in the absence of angiographically demonstrated obstructions in a leg, normal (= score 3) Doppler signals would be found at the groin and at the ankle level. In the case of a significant aortoiliac obstruction both the femoral and the ankle signal would be degraded, whereas obstructive lesions in the periphery of the arteries of the leg would cause a fall of the ankle signal score as compared to the femoral score.

The distribution of the different qualitative (Thulesius) Doppler signal scores over the various angiographic classes of the legs is given in Table A-13. A proportional arrangement of the same data is graphically presented in Figure 10.11. These results are in accordance with the just mentioned theoretical expectations. The legs in which the inflow was not severely compromised (class 0,1 and 2) had normal femoral scores in 78-100 percent of the cases. This

ANGIO CLASS

QUALITATIVE

SIGNAL SCORE

FEMORAL

0

ANKLE
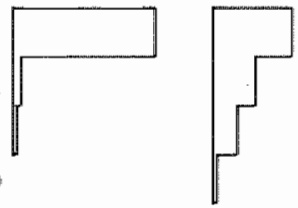

$100 \%$

0

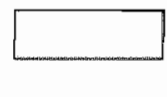

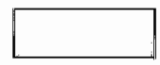
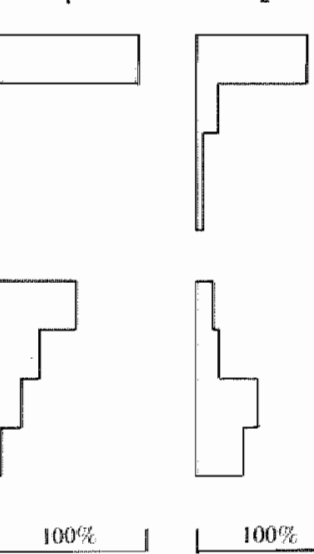

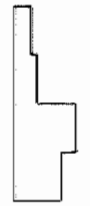

2

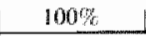

3
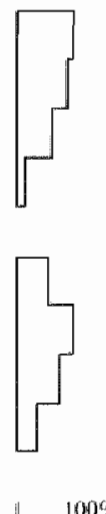

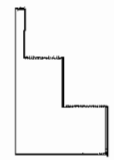

4

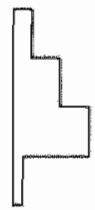

L $100 \%$

FIGURE 10.11

Proportional occurrence of the qualitative (Thulesius) Doppler signal score at the femoral and the ankle tevel by angiographic classification of legs. 
percentage dropped 10 12-36 percent in the legs with high inflow scores (class 3 and 4). Peripheral obstructions caused a shift of the signal scores at the ankle to the lower values as shows a comparison of the figures of class 1 with those of class 2 and of class 3 with those of class 4 .

Between the score of the femoral Doppler signal of the legs and their angiographic inflow score only a poor correlation was found as shown ur Figure 10.12. The mean inflow scores in patients' legs with equal femoral Doppler signal classifications - given in Tabel A-14 and also shown in Figure 10.12 -

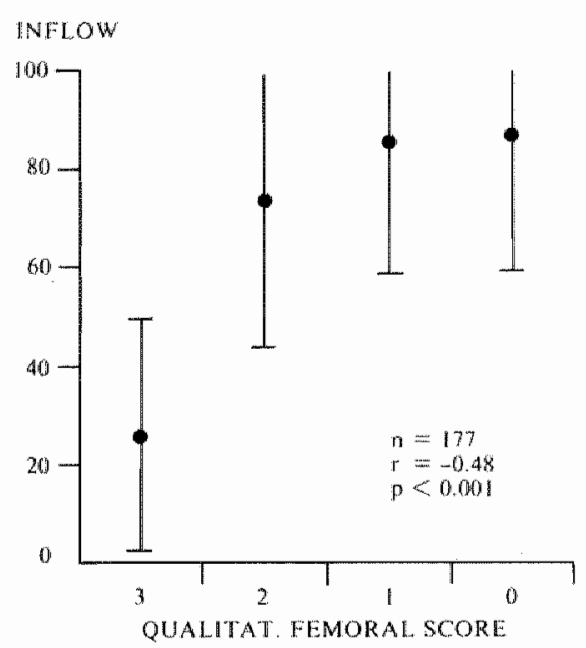

FICURE: 10.12

Correlation between qualitative (Thulesius) femoral Doppler signal score and angiographic inflow score.

Dots represent the mean value of the inflow score, the bars $\pm 1 \mathrm{SD}$.

were not significantly different from each other for the legs with a score 0,1 or 2 $(\mathrm{p}>0.1)$. Only legs with a normal $(=3)$ score had a mean angiographic inflow score which was significantly below that of all the other legs $(p<0.001)$. This separation between legs with a femoral Doppler signal score of 3 on the one hand and legs with all lower scores on the orher hand as far as their aortofemoral obstruction is concerned, is in agreement with reports in which the presence of reverse flow in the femoral Doppler signal is considered an indication of the absence of hemodynamically significant obstruction upstream (FARIS and JAMIESON 1975; BRUININCKX 1976; PERSSON et al 1979).

\subsubsection{Quantitative or numerate Doppler signal parameters}

The digitization of the sonagrams appeared to be a time consuming procedure during which errors could easily be made. Therefore, the possibility to check the digital data by comparing their computer-plotted waveform configuration to the original sonagram, proved to be an indispensable part of the procedures.

Only the calculated parameters of the femoral and the ankle signals were used for further analysis. The parameters of the available aortic signals are not considered for the reasons mentioned in section 10.1.7.1. Ideally, four sets of parameter values could become available for each leg, one set for the ankle at rest ( $=$ ANK parameters) and three for the situations under which the femoral artery had been examined, namely at rest ( = FEM parameters), one minute ( $=$ FEM-1 parameters) and three minutes (= FEM-3 parameters) after thigh occlusion (hyperemia test). For two reasons a few parameter sets were not 
avalable for some legs. One was the exclusion of sonagrams from analysis because of technical reasons. The most important of these was the failure to obtain the post-occlusion signal within the time span allowed by the rest protocol. Very rarely artifacts precluded the proper analysis. These technical reasons occured in none of the FEM signals (0 percent), in 13 FEM-1 signals (5.2 percent), in 6 FEM-3 signals ( 2.4 percent) and in 2 ANK signals (0.8 percent). The other reason why sometimes no parameters could be calculated was the impossibility to record any Doppler sound at all in the case of very low flow or the absence of flow in an artery. This was the case with 5 FEM signals $(2.0$ percent), with 6 FEM- 1 signals and 6 FEM-3 signals (each 2.4 percent) and with 23 ANK signals (9.3 percent). No diagnostic information can be obtained from the situations in which the signall had to be rejected for technical reasons. The fact that no signal could be recorded, definitely is of diagnostic importance. In this section only the results of recordable and acceptable signals will be discussed. Of a total of 992 signals theoretically obtainable from all 248 legs in the study, 931 ( 93.8 percent) were in this category. The non-recordable signals will be discussed in section 10.3.4.

The averages of the various quantitative Doppler signal parameters grouped by the angiographic classes of the legs from which the signals were derived - are given for the four situations (FEM, FEM-1, FEM-3, ANK) in the Tables A-14 to A-23. The same information is diagrammatically presented in the Figures 10.13 to 10.19 thus facilitating a quick reference.

Before proceeding with the discussion of individual parameters attention is given to some general features. A considerable overlap of parameter values for the five angioclasses was found for all the quantitative parameters. It seems impossible to tell any of the classes apart by one single signal parameter. For most paired combinations of classes, however, significant differences $(p<$ 0.001 ) could be found between the mean values of quite some parameters. The classes and the parameters for which these statistical differences were found are summarized in Table A-24 (for FEM signals), Table A-25 (for FEM-1 signals), Table A-26 (for FEM-3 signals) and Table A-27 (for ANK signals). As these tast tables show, no significant differences were attained between the mean values of any parameter for the angioclass 3 and the angioclass 4 legs. This could be an indication that the differentiation between aortoiliac obstructions with and without concomitant distal obstruction (multilevel or single-level disease) might not be possible by the sole use of either femoral or ankle signal parameters. The differences between parameters of legs with mainly important distal obstructions (class 2) and parameters of legs mainly obstructed in their inllow (class 3) did not reach significance for the ANK parameters but did so for quite some parameters of the femoral signals. The legs without any important obstruction (class 0 and 1 ) were different from the legs with obstructions somewhere (class 2 through 4) as far as nearly all ANK parameters were concerned. The majority of parameters of the femoral signals of the non- or only mildly obstructed legs (class 0 and 1) were significantly different from the femoral parameters of the legs with an inflow obstruction (class 3 and 4 ). Differences between femoral signal parameters of "unobstructed" legs (class 0 and 1) and of legs mainly obstructed distally (class 2) were only found for very few of these femoral parameters. These results could indicate that some ANK parameters might be of help in the discrimination between legs with and legs without a significant obstruction but not in the differentiation between legs with mainly aortoiliac and legs with mainly femoropopliteal obstructive disease. This would then be 
analogous to the results of ankle pressure readings (section 10.1.3). In a similar way the femoral signal analys is might then be helpful in differentiating legs with a considerable inflow (or aortoiliac) obstruction from those without such obstructions and possibly also for the discrimination between isolated signiffcant inflow and outflow obstructions. One should however realize that findirng significant differences between the average values of a certain parameter does not implicate its usefulness as a classification criterium for individual legs.

Another feature which was observed without one exception in all classes of legs was the lack of a significant difference $(p<0.001)$ between the mean values of the FEM parameters and the FEM-3 parameters within the same angioclass. The Figures 10.13 to 10.19 demonstrate the tendency of each FEM-1 parameter to be consistently either higher or lower than the corresponding FEM and/or FEM-3 values. However, these differences only reached statistical significance $(\mathrm{p}<0.001)$ for a few parameters and in a few angioclasses and without apparent relation to the classification type. These findings suggest that the alterations of the Doppler signal due to post-occlusion hyperemia may continue for over one minute, even in normals, but will have faded away after three minutes. This is in accordance with the findings of the group of Fronek and Berstein in San Diego (FRONEK et al 1973a, 1973b). Studying the increase of mean femoral flow velocity after an occlusion at the level of the calf - which causes a less severe hyperemic reaction than more proximal occlusions (EHRINGER 1971) - they

$\mathrm{kH} H$

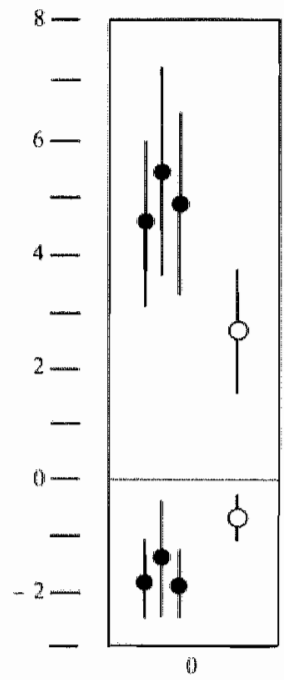

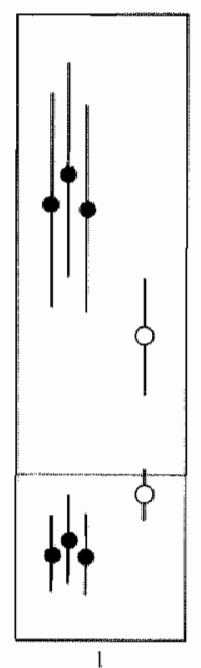

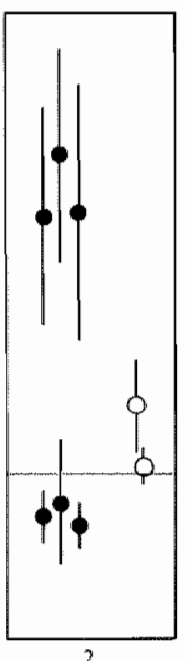

2
MAXFF MAX REW
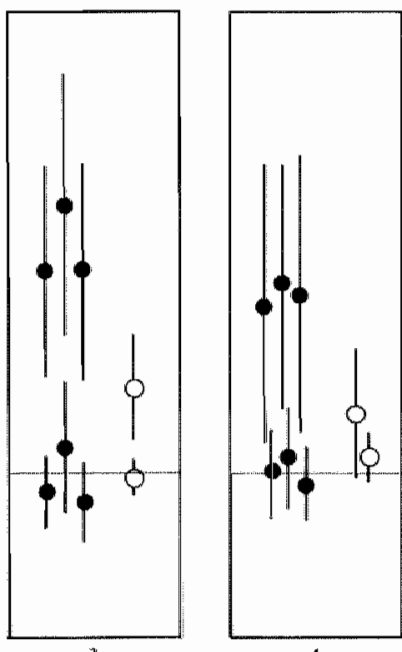

4

FIGURE 10.13

Mean values of the parameters MAX FF and MAX REV for the legs in the different angiographic classes.

The angiographic classes are indicated below the rectangles.

Dots represents the mean values of the femoral signals (left to right: at rest, I minute and 3 minutes post-occlusion), circles represent the values of the ankle signal and the bars \pm 1 SD.

In this combined figure for two parameters the upper row of dots and circles indicates the MAX FF values and the bottom row the MAX REV values. 
found that the time for mean velocity to return to half the increase over the resting value (see also section 6.3.1.) could extend to over one minute in some cases of occlusive disease. It may be logically deduced that the time for a complete return to resting values must have extended beyond one minute quite regularly in their studies.

The Maximum Forward Frequency (= MAX FF) (Table A-15, Figure 10.13)

This parameter was lowered distal to obstructed vascular segments, although only multilevel disease caused a significant reduction of this parameter at the level of the common femoral artery (see Table A-24 through 26). The MAX FF at the ankle was on the average significantly lower in legs with any type of hemodynamically important obstruction (classes $2,3,4$ ) than in legs without such obstructions (classes 0,1 ) (see Table A-27). Only few studies are available to which the obtained results of this parameter might be compared. Japanese investigators found an average maximum forward frequency in normal common femoral arteries of $3.16 \pm 0.66 \mathrm{KHz}$, using $5 \mathrm{MHz} \mathrm{CW}$ Doppler equipment and spectrum analysis of the signals (MATSUO et al 1973; NIMURA et al 1974). Extrapolated to the value which would have been obtained with a 7.6 $\mathrm{MHz}$ equipment, like the one used in this study, the maximum frequency would have been about $4.8 \pm 1.0 \mathrm{KHz}$. This is in good accordance with the result obtained in the present study, namely $4.52 \pm 1.52 \mathrm{KHz}$.

The Mean Forward Frequency (= MEAN FF) (Table A-16, Figure 10.14)
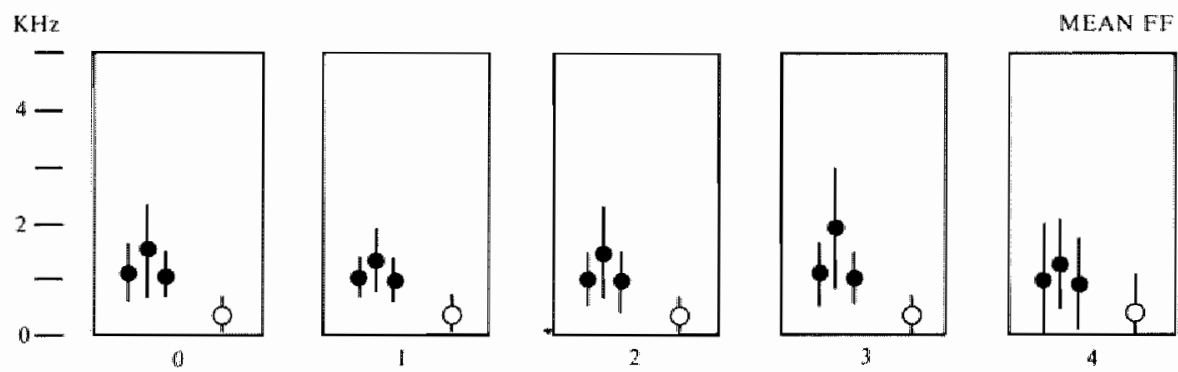

FIGURE 10.14

Mean values of the parameter MEAN FF for the legs in the different angiographic classes.

For explanation see legends to Figure $10,13$.

This parameter - which should not be confused with the mean frequency as it is used in the calculation of the Pulsatility Index (section 6.3.3) and which allows for the reverse flow component, if any - appeared to have nearly the same average value in all angioclasses. It consequently may not be expected to contribute to discrimination between the classes.

The Maximum Reverse Frequency (= MAX REV) (Table A-17, Figure 10.13)

The "highest" (negative) value of this parameter was observed in legs of which the aortoiliac or inflow section was not seriously obstructed, like in the angioclass 0,1 and 2. Severe obstruction caused the well known phenomenon of 
a nearly total disappearance of a reall reverse now component, both at the femoral and at the ankle level (see also section 3.5.2.3). The MAX REV showed of all parameters the most marked differences in mean values between the various classes of legs as demonstrated throughout the Tables A-24 to A-27. The average MAX REV obtained in this study in the normal common femoral artery $(-1.81 \pm 0.71 \mathrm{KHz})$ is slightly, though not statistically significantly, higher than the calculated corresponding value of the Japanese investigators, mentioned in the section on MAX FF, which was $-1.4 \mathrm{KHz}$.

The ratio MAX REV/MAX FF $(=\mathbb{R} / \mathrm{F}$ ) (Table $A-18$, Figure 10.15)
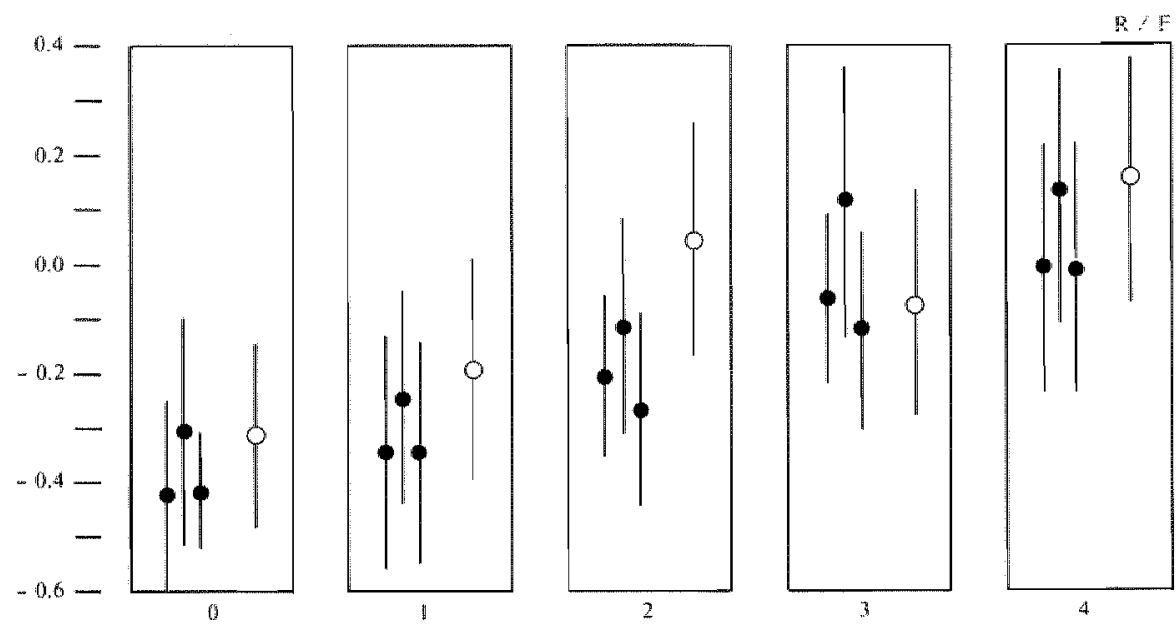

FIGURE 10.15

Mean values of the parameter $R / F$ for the legs in the different angiographic classes.

For explanation see legends to Figure 10.13.

Due to its relation to the parameters MAX REV and MAX FF this parameter reflects the relative contribution of each of these two parameters. The value of $R / F$, which in the present study normally is negative, is shifted to nearby zero or even may become positive in the case of an obstructive arterial lesion proximal to the site of signal recording, mainly rellecting the disappearance of the negative reverse component in the signal. The legs without inflow obstruction (classes $0,1,2$ ) were significantly different from the legs with an aortoiliac obstruction (classes 3,4 ) with regard to their mean $R / F$ values of the femoral signals (see Tables A-24 to A-26). The average normal $\mathbb{E E M} R / F$ value in the present study $(-0,43)$ is very well comparable to the $R / F$ value found by BLACKSHEAR et al (1979) in the common femoral artery with a Pulsed Doppler technique (0.41), the difference in sign only caused by the fact that in Blackshears* study a positive value was given to real reverse signal frequencies and in this study a negative one (section 9.7.5.2b).

The Pulsatility Index (= PI) (Table A-19, Figure 10.16)

Occasionally the calculation of the PI gave rise to a problem which was not referred to in the literature reviewed in section 6.3.3. In signals with a 

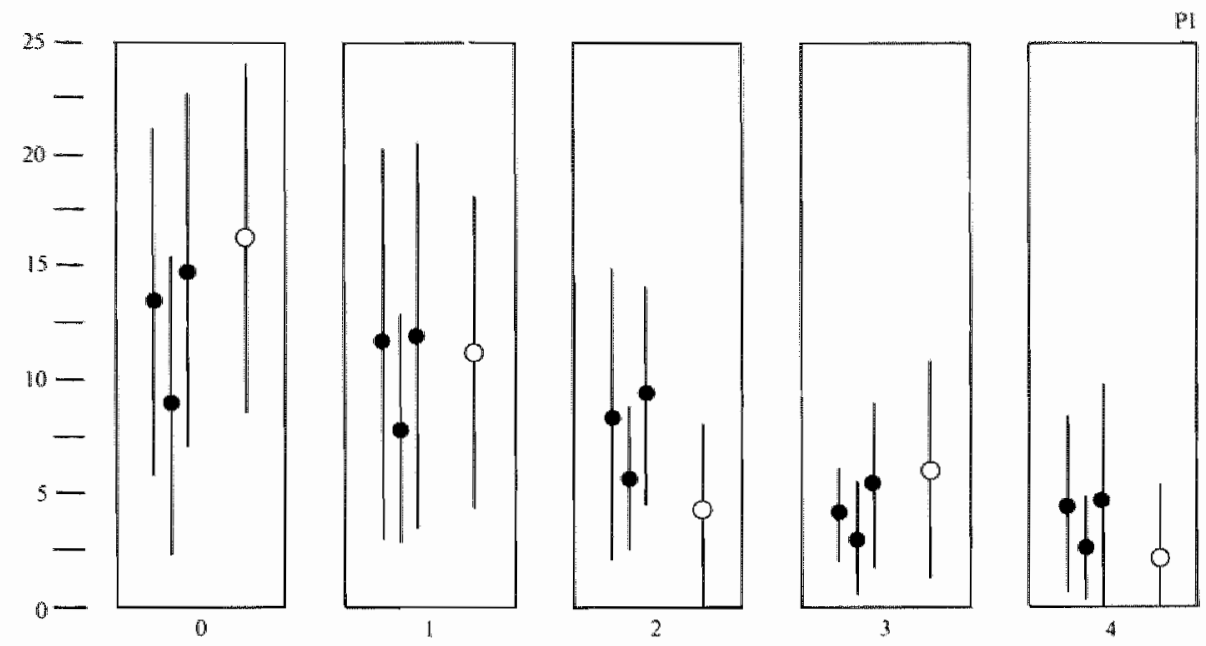

FIGURE 10.16

Mean values of the parameter PI for the legs in the different angiographic classes.

For explanation see legends to Figure 10.13.

considerable reverse component the total area under the maximum frequency envelope of this reverse signal could nearly equal or even exceed the area under the forward evelope, causing a mean signal level which approached to zero or even became negative. In the calculation of the PI the division of the peak-topeak distance by this mean value of approximately zero resulted in extraordinary high (positive or negative) PI values. The mere delineation of the frequency envelope, not allowing for differences in (gray scale) intensity of the sonagram, may lead to this problem. Based on the range of normal PI values from other publications (section 6.3.3) and the PI distribution in the own material, a value of 40 was accepted as the upper limit of a reasonable PI. On the total of 931 signals suitable for parameter calculation 42 ( 4.5 percent) had negative PI"s or a PI above 40. The angiograms of the legs with such high or negative PI's were reviewed. It was remarkable that about half of these signals (47 percent) were obtained from legs of which the angiogram showed a local aneurysm or diffusely pathologically widened arteries. Over one third of the signals ( 36 percent) was obtained in normals whereas only a few signals ( 16 percent) came from symptomatic patients and then all from the legs without significant stenosis. An explanation for the very high or negative PI values is not immediately apparent from these findings, but it is tempting to associate them with the enhanced reverse flow found in aneurysms by others (section 3.5.3). Although the occurence of the extreme PI's was relatively infrequent their values were so far outside the range of the other PI's that the calculation of means and standard deviations for the various classes of legs became meaningless. Therefore, these extreme PI"s were not included in the calculations. leading to Table A-19 and the related Figure 10.16. The very problem of an occasionally occurring extreme PI - which seems inherent to the way PI is defined - reduced its value as a signal parameter considerably. Figure 10.16 demonstrates how in the legs without important inflow obstruction (classes 0,1 , 2) the femoral PI's were on the average higher than in legs with such an 
obstruction (classes 3 and 4 ). These differences were statistically significant ( $\mathrm{p}<$ $0.001)$ (see Table A-24 to A-26). In this study the mean normal PI values ( $\pm S D$ ) for the common femoral artery $(12.6 \pm 7.5)$ and for the pedal/ankle arteries $(16.4 \pm 7.8)$ were slightly higher than comparable values obtained by GOSLING and KING (1974b) with their peak-to-peak PI (common femoral artery 5-10, pedal arteries 7-15) (see section 6.3.3). In general the figures are quite of the same magnitude as the figures mentioned in section 6.3 .3 for normal values.

The Upslope and Downslope Times (= UST(H), UST (DER), DST) (Tables A-20 to A-22, Figures $10.17,10.18$ )

$\mathrm{MSEC}$

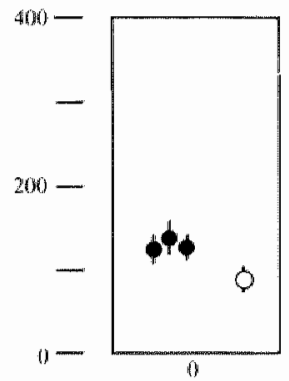

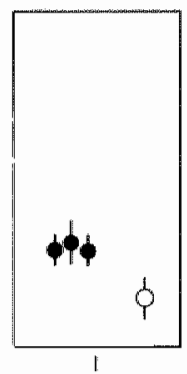

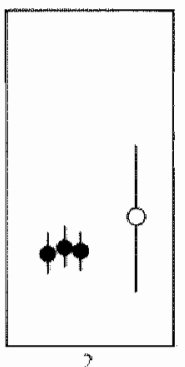

UST (DER)
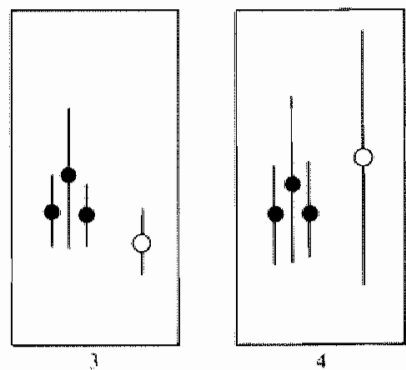

FIGURE $\| 0.17$

Mean values of the parameter UST (DER) for the legs in the different angiographic classes.

For explanation see legends to Figure 10.13.

MSEC

DST
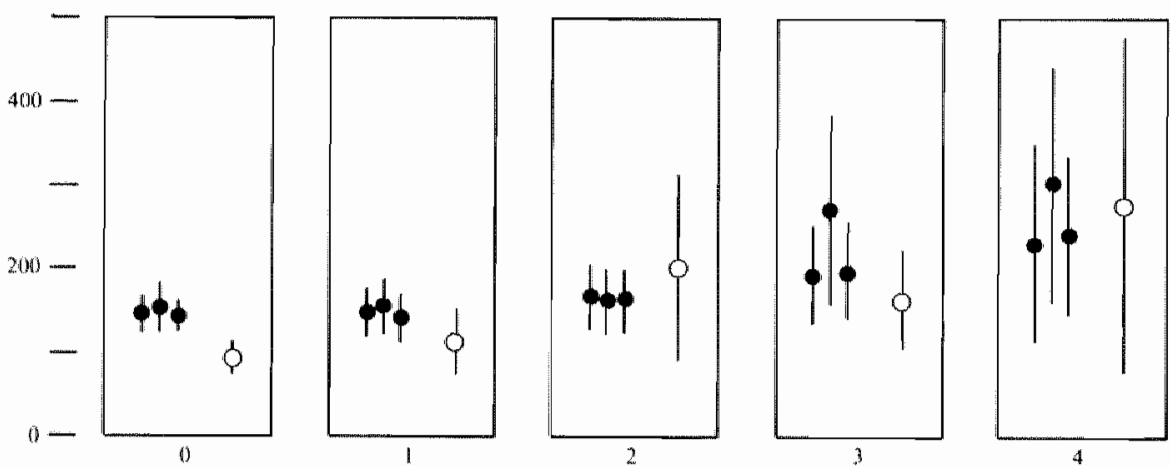

FIGURE 10.18

Mean values of the parameter DST for the legs in the different angiographic classes.

For explanation see legends to Figure 10.13.

Comparison of the Tables A-20 and A-21 makes it clear that the determination of the UST of the sonagram by hand (UST(H)) gave quite the same outcome as its determination by the more complicated computer determination (UST(DER)). 
The differences usually did not exceed a few milliseconds and were negligible in comparison to the standard deviations. Both at the common femoral and at the ankle level the mean UST(H), UST(DER) and DST of signals recorded in the presence of an important upstream obstruction were all significantly prolonged in comparison to similar time spans in signals from vessels without such obstructions. The finding by NICOLAIDES et al (1976) (section 6.3.2) that obstructive disease confined to the vessels distal to the common femoral artery caused a prolonged downstroke period of the Doppler signal curve was not confirmed in this study. No significant differences in femoral DST were found between class 0 and/or class 1 legs on the one hand and class 2 legs on the other hand. The normal FEM UST"s obtained in this study were in the same order of magnitude as the values reported by DILLEY and FRONEK (1978). A comparison of the results of the "pulse decay times" of these authors with the DST"s in the present study is not meaningful because of a difference in definition of the parameters.

The Null Fraction (= NULL) (Table A-23, Figure 10.19)
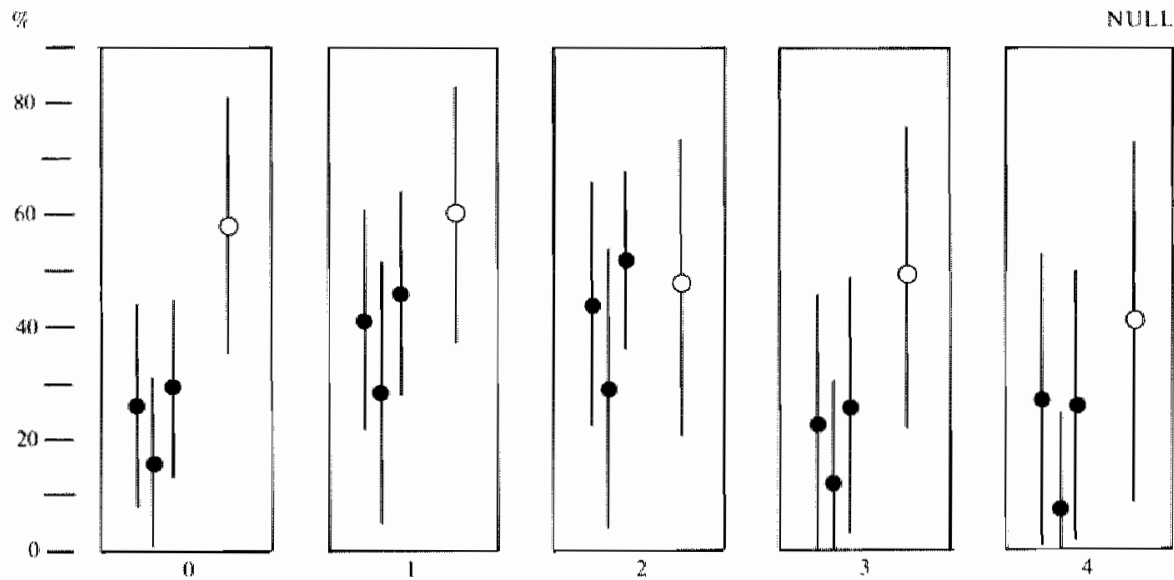

FIGURE 10.19

Mean values of the parameter NULL for the legs in the different angiographic classes.

For explanation see legends to Figure 10.13

This parameter was thought to be a measure of the degree of "briskness" of the Doppler signal. It, therefore, would ideally be low in nearly continuous (legato) signals as found downstream of obstructions, and high in the more normal (staccato) signals. Part of this concept indeed was recognized in the results of the femoral NULL measurements. The legs with important inflow obstructions had lower NULL values on the average than the unobstructed patients" legs. But in the Doppler signals of the normal test population a low NULL value was usually also found in the registrations of the common femoral artery. This was probably caused by the frequent occurence of a considerable diastolic forward component in the signal. At the ankle the Doppler signals of both patients and normals nearly always exhibited a discontinuous, staccato, character which was reflected in relatively high NULL, values without significant differences between 
the various angioclasses. Since the NULL Fraction was not reported anywhere else in literature a comparison with other studies is not possible.

\subsection{SELECTION OF PARAMETERS FOR FURTHER STATISTICAL} ANALYSES

For an assessment of the relative value of the various noninvasive tests the ranking and classification program described in section 9.8 was used. The outcome of such a procedure, of course, depends largely on the sought for classification of legs. A certain parameter, for instance, may give an optimal discrimination between legs with and without angiographical abnormality but can fail to distinguish between different locations of obstruction or fail to grade the degree of aortoiliac obstructive disease.

Three different a priori classifications were used in the evaluation of the tests. All were based on the angiographic classes described in section 9.7.1.

- The first a priori classification consisted of angiographically "normal" and "abnormal" legs. A leg was considered to be "normal" if both the inflow and outflow scores of the leg were $<50$ and "abnormal" if one or both of these scores were $>50$. Consequently the "normal" legs had either angiographic classification 0 or $\mathbb{1}$ and the "abnormal" legs had an angiographic classification of 2,3 or 4 .

- The second a prioriclassification was based on the segmental distribution of important obstructions, distinguishing between legs without important obstructions (class 0 and/or 1), with obstructions mainly confined to the aortoiliac or inflow section (class 3 ), with obstructions mainly limited to the arteries distal to the groin (class 2) or with obstructions at both levels (class 4). In this classification the legs of the healthy subjects (class 0 ) could either be considered separately, making the classification a 5-classes problem or grouped together with the class 1 legs thus reducing the problem to a 4 groups classification.

- The third classification distinguished between legs of which the inflow section appeared to have no important obstruction on angiography ("patent") and legs with an obstruction in that section ("obstructed"). The "patent" group included angioclass 0,1 and 2 legs, the "obstructed" group angioclass 3 and 4 legs. This very differentiation constituted the aim of the present study and, therefore, will be discussed in more detail (section 10.3 .3 ) than the other two classifications (sections 10.3.1 and 10.3.2).

Some parameters were excluded from the ranking procedure since no contribution to any of the classifications could be anticipated on the basis of the results presented in section 10.1 . These parameters were the presence or absence of iliacofemoral bruits at rest and after exercise (section 10.1.2), the claudication and maximum walking time during the treadmill walking test (section 10.1.4) and the aorto-femoral transit time values (10.1.6).

The quantitative Doppler signal parameters were also submitted to a preselection. As discussed in section 10.1.7.3 under "Pulsatility Index" (PI), the value of the PI in 4.5 percent of all Doppler signals accepted for analysis was far outside a reasonable range, making t-test analysis and ranking meaningless. This was shown in preliminary ranking studies in which the PI's of the Doppler signals, recorded in the four situations FEM, FEM-1, FEM-3 and ANK for details see section 10.1.7.3), consistently ranked lowest in the above discussed 5groups classification. After exclusion of the signals with extreme Prs, ranking 
was repeated. Since the program ISPAHAN was unable to cope with "missing parameters" also the corresponding other quantitative signal parameters had to be excluded from ranking which considerably reduced the total number of classifiable legs. In this new ranking the PI's of the FEM, FEM-1 and the FEM-3 again had the lowest discriminative power when used in the 5-groups classification, the "normal-abnormal" classification and the discrimination between inflow "patency" and "obstruction". Only the PI of the ankle signal scored 5th among the parameters in the "normal-abnormal" classification. It, however, added nothing to the percentage correct classifications which was already reached with the use of the 4 other, higher scoring, ankle signals parameters. For these reasons the $\mathrm{PI}$ - as a non relevant parameter - was excluded from the final ranking and classification studies in order not to reduce unnecessarily the total number of legs available for statistical analysis.

In another analysis the value of quantitative parameters of the Doppler signal of the femoral artery at rest (FEM) was compared to that of the parameters of the signals one and three minutes after release of the thigh occlusion (FEM- 1 and FEM-3). As already discussed in section 10.1.7.3 no significant differences between any of the mean values of the FEM and FEM-3 parameters and only a few differences between FEM-1 and either FEM or FEM-3 parameters were found for the various angiographic classes. This gave rise to doubt about the extra information to be gained from this type of hyperemia measurements. The quantitative Doppler signal parameters were grouped together in three different ways: FEM parameters together with ANK signal parameters, FEM together with FEM-1 and ANK parameters and in the third place ANK parameters with FEM-1 and FEM-3 parameters. All parameters of each of these three combination groups of parameters were ranked according to their discriminative power in the classification of the earlier mentioned 5 a priori groups and the proportion of correct classifications was calculated. The outcome of this analysis was that the combination of FEM and ANK parameters only scored higher percentages correct classifications than combinations containing FEM-1 and FEM-3 parameters. In the combined parameter groups of FEM and FEM-1 parameters the FEM parameters also generally scored better than the FEM-1 parameters. It, therefore, was concluded that the parameters of the femoral signals after thigh occlusion gave no improvement of the classification of the legs in comparison with the parameters obtained at rest. Hence, only the femoral and ankle signal parameters obtained at rest were used for further analyses.

The noninvasive test parameters selected for the final statistical analyses were:

- the scores of the pulsations of the common femoral, the popliteal and the ankle arteries.

- the thigh and ankle pressure indices at rest.

- the classification of the ankle pressure response to exercise.

- the qualitative (Thulesius) Doppler signal scores of the common femoral artery and (one of) the ankle arteries at rest.

- the set of quantitative parameters (PI excluded) of the Doppler signal of the common femoral artery and the ankle artery both obtained at rest.

A detailed listing of this selection of parameters is found in Table 10.1 .

In the series of parameters a hierarchical arrangement may be recognized which is related to the way these parameters are applied in daily clinical practice: Pulse palpation is practiced by all physicians. Segmental systollic pressure 
TABLE 10.1

Listing of parameters seleded for ranking and classification procedures.

\begin{tabular}{lll}
\hline PARAMETER TYPE & PARAMETER DESCRIPTION & ABBREVIATION \\
\hline Clinical parameters & Pulsation score of comm. fem. art. & PULS FEM \\
& $\begin{array}{l}\text { Pulsation score of popliteal art. } \\
\text { Pulsation score of ankle artery }\end{array}$ & PULS ANK \\
Pressure parameters & Thigh pressure index at rest & TPI \\
& Ankle pressure index at rest & API \\
& Ankle pressure response te exercise & RESPONSE \\
Qualitative signal & Thulesius signal score femoral art. & FETHU \\
parameters & Thulesius signal score ankle art. & ANTHU \\
Quantative signal & Max. frequency of forward signal & MAX FF \\
parameters* & Max frequency of reverse signal & MAX REV \\
& Mean frequency of forward signal & MEAN FF \\
& Ratio of MAX REV/MAX FF & R/F \\
& Upslope time (hand-meastrement) & UST (H) \\
& Upslope time (derivative-measurement) & UST (DER) \\
& Downslope time & DST \\
& Null or zero fraction & NULL \\
\hline
\end{tabular}

* The addition "(F)" to the parameter names means a parameter of the signal of the common femoral artery and "(A)" denotes a parameter of an ankle artery. E.g. $\operatorname{MAXFF}(F)$ and $R / F(A)$.

readings might easily be performed by every physician but will usually be considered only necessary in the examination of vascular patients by medical specialists. The ank le pressure response to exercise is generally determined only in a small group of patients on circumscribed indications; it requires equipment and technical assistance which usually is only available in specialized vascular clinics. In this last setting also the physicians interested in the quality of peripheral arterial Doppler signals will be found. Until now the performance of exact measurements and calculations on Doppler signals remained restricted to a few specialized vascular laboratories. The ultrasound section of the usual vascular laboratories will mosily provide only systolic pressure data and a simple signal registration for visual assessment.

This hierarchical structure of tests was reflected in the way in which the various parameters were grouped together in four different "sets". Throughout the further ranking and classification analyses the following arrangement of tested parameters will be maintained:

SET 1 represented the noninvasive test facilities available to a physician assisted

by a simple ultrasound laboratory. This set included as parameters: pulse scores, thigh and ankle pressure indices at rest, the type of ankle pressure response to exercise and the qualitative scores of the Doppler signals.

SET II represented the facilities of the physician who has a more sophisticated type of ultrasound laboratory at his (her) disposal with the possibility to calculate the selected numerate signal parameters.

This set consisted of all parameters of SET I plus the quantitative Doppler signal parameters. 
SET III represented the position in which a technician in a sophisticated vascular ultrasound laboratory would find him (her)self when trying to classify patients" legs on the basis of Doppler tests without knowledge of the results of physical examination performed (or still to be performed!) by the physician. This situation is no longer uncommon nowadays. This set comprised all parameters of SET II without the three pulsation scores.

SET IV consisted of the quantitative Doppler signal parameters only. It was included to allow an answer to the questions put in section 1.2 in the light of the aim of the present study.

A detailed summary of all parameters in each of these four sets is given in Table 10.2 .

TABLE 10.2

Grouping of test parameters in Cour different parameter sets.

\begin{tabular}{llll}
\hline SET I & SET II & SET III & SET IV* \\
\hline PULS FEM & Combination of all & TPI & MAX FF \\
PULS POP & parameters of & API & MAX REV \\
PULS ANK & SET I + SET IV & RESPONSE & MEAN FF \\
TPI & & FETHU & R/F \\
API & & ANTHU & UST (H) \\
RESPONSE & & + & UST (DER) \\
FETHU & & All parameters & DST \\
ANTHU & & OF SET IV & NULL \\
\hline
\end{tabular}

* SET IV includes the listed parameters for both the comm.fem.art. (F) and the ankle art. (A).

\subsection{DIAGNOSTIC RESULTS WITH THE SELECTED PARAMETERS}

Since the program ISPAHAN (section 9.8) which was used for the analyses described in the sections to follow, was unable to handle "missing parameters", only those legs were used in the computations of which both the angiographic inflow and outflow scores and the values for all parameters described in Table 10.1 were known. Exactly 200 legs fulfilled these conditions and thus were available for the analyses.

\subsubsection{General diagnosis of arterial obstruction of the legs}

In order to assess the value of the various parameters of the different sets for the noninvasive distinction between angiographically "normal" and "abnormal" legs, the parameters were submitted, set by set, to the ranking procedure while using the "normal-abnormal" a priori classification (section 10.2). The first four parameters, ranked according to discriminative power, for the SETS I. II and IV are given in Table 10.3. From this table the importance of pulse palpation and the determination of the ankle pressure index at rest for the distinction between normal and abnormal legs becomes apparent. For the recognition of obstruction in the arteries of the leg the first-ranking numerate Doppler signal parameter (MAX REV (F)) was inferior to popliteal pulse palpation and ankle pressure measurement, as shown in the analysis of the 
TABLE 10.3

Ranking of the parameters of the sets according to their power to discriminate betwen "normal" and "abrormal" legs.

\begin{tabular}{|c|c|c|}
\hline $\mathrm{SET}$ & SET II & SETIV \\
\hline PULS POP & PULS POP & MAXREV(F) \\
\hline API & $\mathrm{API}$ & $\operatorname{MAXFF}(A)$ \\
\hline PULS FEM & $M A X R E V(F)$ & $\operatorname{DST}(\mathrm{A})$ \\
\hline RESPONSE & PULS ANK & DEST $(F)$ \\
\hline
\end{tabular}

combined use of tests (SET II, Table 10.3). Using a popliteal pulsation score $\leqslant 2$ as criterion of angiographic "abnormality", 88 percent correct classifications could be reached at. This figure could not be improved substantially by combining the pulsation score with one of the lower ranked parameters in a classification rulle. About the same proportion of correct diagnoses could be achieved by the combined use of the quantitative parameters MAX REV (F) and MAX FF (A) in one classification formula. These findings seem te permit the conclusion that the mere establishment of the presence or absence of important obstructions in the arterial system of the lower limbs requires only a careful palpation of the popliteal pulse and ankle pressure readings at rest. A disadvantage of the use of the pulsation score as diagnostic criterion is its reported poor reproducibility and subjective character (section 2.2.1). The ankle pressure measurement is more objective and is generally accepted as criterion for that reason (KITSLAAR 1981 and section 5.5.1).

\subsubsection{Segmental diagnosis of arterial obstructions}

In the assignment of legs to one of the four a priori classes which represent the various types of segmental distribution of angiographic obstructions (section 10.2), an about 80 percent correct classification score could only be achieved by the combined use of 5 to 6 parameters. This implicated the use of multiple classification rules. In a similar classification in which the legs of the healthy test persons were separated from the patients' legs and grouped apart in a fifth a priori class (5-groups classification, section 10.2) a highest proportion of correct classifications of only 68 percent could be achieved by the simultaneous use of 7 parameters. In both classifications the addition of further parameters to the four first-ranking ones did not substantially increase the total number of correct classifications. Classifications based on the combined use of multiple variables may be interesting from a statistical-methodological point of view but will probably not gain acceptance in routine clinical practice. The relative importance of the four first-ranking parameters of SET I, II and IV as far as the 4and 5-groups classification was concerned can be read from Figure 10.20. In this figure also the total percentage of correct classifications for the use of either one or two, three and four parameters in combination is given. At the fourth parameter the curve representing the percentage correct classifications levels off. The examination of the peripheral pulsations which appeared to be important for the distinction between "normal" and "abnormal" limbs (section 10.3.1), also occupied the first place among the tests in the diagnosis of the segmental distribution of obstructions. These parameters were followed in 


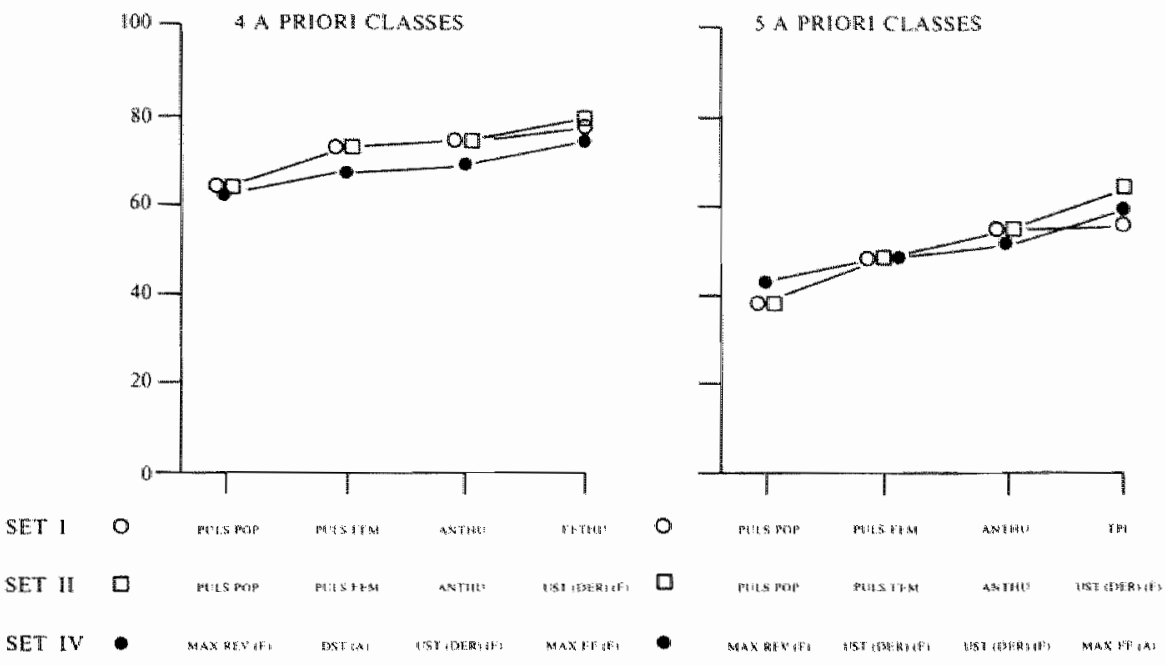

PARAMETERS EMPLOYED - RANKED ACCORDING TO IMPORTANCE

FIGURE 10.20

Results of the segmental diagnosis of arterial obstruction in the legs.

Results are given for different parameter sets and the simultaneous use of successively increasing numbers of parameters.

The parameters are ranked in order of discriminative power decreasing from left to right. For abbreviations see Table 10.1

importance by qualitative and quantitative signal parameters and not by pressure measuremenis like in the "normal-abnormal" analysis. It is also evident from Figure 10.20 that both the 4-and the 5-groups classifications were performed by about the same parameters. So, apparently none of the tested parameters seems to allow an accurate differentiation between the patients' legs with only minor obstructions (class 1) and the limbs of healthy test subjects (class 0). Further evidence for this was the fact that assignment of class 0 legs to class 1 and vice versa nearly completely accounted for the differences in correct classification percentages between the 4-groups and the 5-groups classification (see Figure 10.20).

\subsubsection{Diagnosis of aortoiliac obstructive disease}

10.3.3.1. Classifying parameters, classification rules and diagnostic accuracy, specificity and sensitivity

The result of the classification of the legs in those with a "patent" and those with an "obstructed" inflow section (section 10.2) was worked out more elaborately than the results discussed above, since it was the very question of the present study how to diagnose by noninvasive tests an important aortoiliac or inflow obstruction. The ranking order within the four sets of parameters according to their power to discriminate between "patency" and "obstruction" of the inflow 
Ranking of the parameters of four sels according to their power to discriminate between arriollac "patency" and "obstruction".

\begin{tabular}{llll}
\hline SET & SET II & SET $\| I$ & SET IV \\
\hline PULSFEM & PULS FEM & TPI & MAX REV(F) \\
TPI & TPI & UST $(H)(F)$ & UST (DER)(F) \\
PULS POP & UST (H) $(F)$ & MAXREV $(F)$ & R/F (F) \\
ANTHU & MAXREV $(F)$ & ANTHU & MAX FF (F)
\end{tabular}

section is presented in Table 10.4 in which only the first four parameters of each set are given. It is evident that clinical parameters and segmental pressure readings surpassed the qualitative and quantitative Doppler signal parameters in importance. If the choice was between qualitative and quantitative parameters of the Doppler signal (like in SET II and SET III) quantitative parameters prevailed.

For each set of parameters, classification rules - which had the form of an arithmetical formula - were formulated for the combined use of the first two, three and four parameters of the various parameter sets respectively. These classification formulas were based on the Fisher directions or vectors of the parameters (section 9.8). The outcome of such a classification formula after insertion of the parameter values of an individual leg was a single figure (Q), which might be called a "classification quotient". If solely the first-ranking parameter was used the very value of that parameter was at the same time the $Q$ value. Depending on wether this classification quotient $Q$ for a leg was above or below a chosen threshold value, the leg was allocated to the "patent" or "obstructed" group of legs. Comparison of the thus predicted classification of limbs to the actual or a priori classification (= angiographic classification), using a variety of arbitrarily selected $Q$ values, allowed the determination of an optimal threshold for $\mathrm{Q}$ by means of $\mathrm{ROC}$ analysis (see section 9.8 and Figure 10.21). The combined use of the first two parameters of the different sets in their classification rules improved the diagnosis of inflow obstruction in comparison to the diagnosis by means of the first-ranking parameter alone. It appeared that the combined use of more than two parameters of the different parameter sets did not improve diagnostic accuracy, specificity and sensitivity anymore. Therefore, only the classification rules for the first two parameters were further used.

The classification formulas for the various parameter sets, described in section 10.2 and in Table 10.2, are presented in Table 10.5. In that same table also the optimal threshold values of $Q$, as determined by ROC analysis, for the first parameter alone and the combination of the first and second-ranking parameter, are specified for each set. The diagnostic accuracy, specificity and sensitivity in the classification of individual limbs of this study, obtained by application of the classification rules with the selected $Q$ values (see Table 10.5 ) are summarized in Table 10.6. The resulting percentages for the different test combinations are of about the same magnitude and only slightly in favor of the combination of femoral pulse palpation and thigh pressure measurement. This is again an indication of the relative importance of clinical examination in the diagnosis of arterial disease (section 10.3.1 and 10.3.2). 

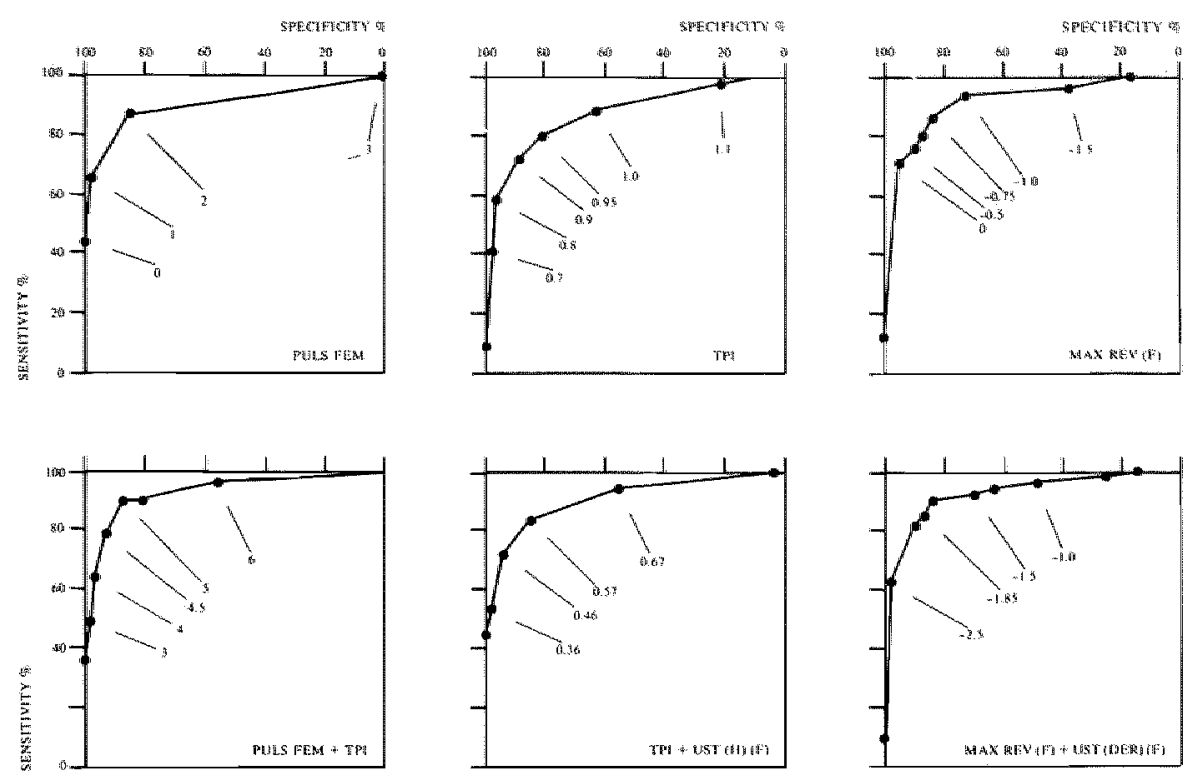

FIGURE 10.21

ROC curves for different classification formulas.

Various threshold criteria of the classification coefficients Q for the six classification rules of Table 10.5 are indicated.

TABLE 10.5

Classification rules and classification coefficients for the first two parameters of the various sets of parameters in the diagnosis of aortoiliac obstruction.

\begin{tabular}{lll}
\hline $\begin{array}{l}\text { PARAMETER } \\
\text { SET }\end{array}$ & CLASSIFICATION RULE & $\begin{array}{l}\text { CRITERION FOR INFLOW } \\
\text { SCORE }>50\end{array}$ \\
\hline $\mathrm{I}=\mathrm{II}$ & $\mathrm{Q}=$ PULS FEM & $\mathrm{Q} \leqslant 2$ \\
& $\mathrm{Q}=\mathrm{PULS}$ FEM $+3 \times$ TPI & $\mathrm{Q} \leqslant 5$ \\
$\mathrm{III}$ & $\mathrm{Q}=\mathrm{TPI}$ & $\mathrm{Q} \leqslant 0.95$ \\
& $\mathrm{Q}=\mathrm{TPI}-0.003 \times$ UST $(\mathrm{H})(\mathrm{F})$ & $\mathrm{Q} \leqslant 0.57$ \\
$\mathrm{IV}$ & $\mathrm{Q}=$ MAX REV $(\mathrm{F})$ & $\mathrm{Q} \geqslant-0.75$ \\
& $\mathrm{Q}=-\operatorname{MAX} \operatorname{REV}(\mathrm{F})-0.02 \times$ UST (DER) (F) $\mathrm{Q} \leqslant-1.85$ \\
\hline
\end{tabular}

TABLE 10.6

Accuracy, specificity and sensitivity of the first two parameters of the various parameter sets in the diagnosis of aortoiliac obstruction.

\begin{tabular}{|c|c|c|c|c|}
\hline \multicolumn{2}{|c|}{ PARAMETER(S) } & \multirow{2}{*}{$\frac{\text { ACCURACY } \%}{85.0}$} & \multirow{2}{*}{$\frac{\text { SPECIFICITY } \%}{84.3}$} & \multirow{2}{*}{$\frac{\text { SENSITIVITY of }}{87.3}$} \\
\hline $\mathrm{SET} \mathbb{\|}=\mathbb{I I}$ & first & & & \\
\hline & first two & 87.9 & 87.6 & 89.0 \\
\hline \multirow[t]{2}{*}{ SET MII } & first & 80.0 & 80.2 & 79.1 \\
\hline & first two & 84.0 & 84.2 & 83.3 \\
\hline \multirow[t]{2}{*}{ SET $\mathbb{V}$} & first & 83.2 & 82.5 & 85.4 \\
\hline & first two & 85.5 & 85.8 & 84.3 \\
\hline
\end{tabular}


In the series PULS FEM, TPI, MAX REV and UST(DER or H) the PULS FEM is a parameter which is purely subjective and strongly dependent on the examiner. Moreover, only a few discrete parameter values are possible. Scoring the pulsation strength one point higher or lower than a nother examiner would doa situation which will frequently occur, see section 2.2.1-causes an enormous shift on the ROC curves (see Figure 10.21). This will reduce the value of the first two parameters of SET I and II when used by different physicians or technicians for an objective grading of aortoiliac obstruction. Pressure measurements (TPI) are notoriously reliable, also in the hands of different investigators (section 5.1). The MAX REV of Doppler signals is operator-dependent as it will change considerably with different probe/vessel angles (section 4.4). On theoretical grounds changes of the upslope time of Doppler signals measured by hand (UST(H) due to changes of the amplitude of the Doppler signal curvecaused by probe manipulation, will remain small compared to the accuracy of the determination itself, provided the artery is completely insonated (HUMPHRIES et al 1980, see also section 6.3.2).

\subsubsection{Discussion of false-positive and false-negative classifications}

For the purpose of the following analyses the classification rules for two paramelers in conjunction with their optimal $Q$ criteria, as defined in the previous section 10.3.3.1, were applied to all legs of which the inflow score and the two parameters in question were available. Also legs with unclassifiable outflow sections and missing extraneous parameters were now included. This brought the total number of legs available for these classifications at 233,33 more than were used for the deduction of the formulas and criteria. Comparison. of the predicted with the a priori classifications learned wich legs were falsely classified as positive $(=$ predicted inflow score $>50$, a priori score $\leqslant 50)$ or negative $(=$ predicted inflow score $\leqslant 50$, a priori score $>50)$. Angiograms, pressure data, original sonagrams and physical examination of the concerned leg were reviewed in order to find reasons for these false classifications. Special attention was given to aneurysms in the inflow pathway on the anglogram. Aneurysms might cause flow disturbances (section 3.5 .3 ), influencing the results of pressure measurements or signal analysis and thus of classification. In the same way the occurrence of stenoses of $41-50$ percent diameter reduction was noted, especially if these diameter reductions were accompanted by an increased local transparancy of the contrast, an aspect not considered in the a priori classification. In view of the known angiographic underestimation of a ortoiliac disease (section 2.2.2) such stenoses mighl well be an indication of more serious obstruction than the a priori score suggested.

The classification by means of the femoral pulsation score and the thigh pressure index (PULS FEM and TPI, SET I=II) resulted in $2 \|$ legs falsely classified as positive. Of these legs 4 ( 19 percent) had an inflow score above 40 of which 2 had concomitant occlusion of the superficial femoral artery (SFA). Occlusion of the SFA together with less severe proximal stenosis (10-40 percent, average 31.5 percent) was found in 11 of these legs falsely classified positive ( 52 percent). The total proportion of legs with SFA occlusions in this specific group of legs was 61.9 percent. Among the 6 remaining false-positive legs one had a large aortic aneurysm and iliac stenosis of 25 percent, whereas the other 5 legs had aortoiliac stenoses ranging from 20 to 40 percent only. One of these legs ( 20 percent stenosis) was reexamined with oblique angiographic views some days after the 
first TLA and a very circumscribed 55 percent diameter reduction in anteroposterior direction was found in the common diac artery. Legs of healthy reference persons were not classified falsely positive by means of the SET $I=I I$ classifier.

Using the thigh pressure index and the upslope time of the femoral Doppler signal (TPI and UST(H)(F); SET III) as classifiers, 32 legs were falsely classified as positive. Eleven (34.4 percent) of these legs had an inflow stenosis between 41 and 50 percent, and of these legs again 7 had a SFA occlusion as well. An occlusion of the SFA in the presence of less severe inflow stenoses (estimated between 0 and 40 percent, average 15 percent) was 11 times found in this series of falsely classified limbs (34.4 percent). The total proportion of legs with angiographically occluded SFA's in this specific group was 56.2 percent. Of the 10 other legs 5 had angiographic evidence of stenoses in the aortoliac pathway ranging from 25 to 37 percent, in one leg in an extremely elongated iliac artery, whereas one other leg had an absent profunda femoris artery and a 60 percent origin stenosis of the SFA. The remaining 5 legs were legs of normal healthy test persons of both young and old age.

With the two qualitative Doppler signal parameters (MAX REV (F) and UST(DER) (F); SET IV) the number of false positive classifications was 25. Seven of these legs ( 28 percent) with inflow scores of $41-50$ percent might possibly be considered true positives and 3 of these legs had also an SFA occlusion. An SFA occlusion in the presence of proximal scores of less importance (25-40 percent, average 35.5 percent) was found in 4 other legs. The proportion of limbs with an SEA occlusion herewith totalled 28 percent. The remaining 14 falsely classified legs were 7 normal legs of test subjects of both sexes and age groups, 3 legs with minor inflow stenoses which were also wrongly predicted by the "SET III" classification rule, the two legs of a patient with an extremely dilated aortic bifurcation prosthesis without apparent obstructions and one leg with a huge aortic and common iliac aneurysm.

Six legs were wrongly classified positive by all three classifiers.

There were 5 false-negative classifications with the SET I=II classifier, 4 with the SET III and 7 with the SET IV classifier. Three legs were wrongly classified by all three tests: 2 legs with inflow scores of 68 and 72 percent and one leg with an aortic and common iliac aneurysm with a stenosis estimated at 69 percent. The SET I classifier had missed two other legs with stenoses of 53 and 66 percent, both with concomitant SFA occlusion. Both the SET III and the SET IV rules classified another leg with an inflow stenosis of 56 percent falsely negative. The SET IV classifier had falsely classified three legs negative which even had complete occlusions of the external illac arteries. Review of the original femoral sonagrams on which the classification was based, learned that these were probably registrations of some collaterals in the femoral region and of a poor quality. They better would have been excluded from analysis and booked as un recordable (see section 10.3.3.3).

Some conclusions can be drawn from these observations:

- Among the legs falsely classified positive by means of SET I=II and SET III classifiers, in which thigh pressure determination plays an important part, high proportions of legs with occlusion of the superficial femoral artery were found (61.9 and 56.2 percent). These proportions are significantly higher ( $p$ $<0.015$ ) than the 28 percent of such legs in the group falsely classified by means of numerate signal parameters only (SET IV). This might be an indication that the effect of superficial femoral artery occlusion, which 
reduces the specificity in the detection of aortoiliac obstructions for thigh pressure measurements as was shown again in this analysis (see also section 5.5.1,8.1 and 10.1.3), is less manifest for the Doppler signal parameters.

- In 13 percent of all false-positive and negative classifications an aneurysm was found on the angiogram of the legs concerned. Since the total proportion of aneurysmatic segments in all angiograms was 21.8 percent (section 10.1.1) it is very unlikely that aneurysms played an important part in disturbing the diagnosis of aortoiliac obstruction by the described tests. They certainly interfered with the a priori estimation of stenosis percentages.

- Classifications merely based on the Doppler signal are disturbed if based on registrations of poor quality.

- With the classifiers containing qualitative Doppler signal parameters (SET III and IV) some normal legs were wrongly classified positive. Further analysis of these cases learned that such classifications were caused by upslope times (UST) of the curves which were longer than the average normal value, although the signal itself had a quite normal appearance. No explanation was found for this phenomenon.

\subsubsection{Non-recordable Doppler signals}

All analyses up to this point were performed on data of legs for which Doppler signals could be registrated and analysed. For those few cases in which no usable signal could be reconded in spite of a thorough search for it (section 10.1.7.3), the diagnostic value of this fact was analysed. Five femoral signals were unrecordable. In 4 cases the leg concerned had angiographic evidence of total occlusion of the illac or common femoral arteries. In one leg the inflow score was 37 but the groin was severely scarred and the femoral pulse not palpable due to a previous vascular operation. Non-recordable Doppler signals at the ankle occurred in 23 legs. The angiograms of these limbs showed occlusion at two levels (both inflow and outfow scores 100) (30.4 percent), occlusions in the inflow and less severe stenosis more distally (17.4 percent), total occlusions in the outflow section and less severe obstructions in the inflow (13.1 percent) or subtotal, but hemodynamically significant, obstructions at one or two levels (39.1 percent). The very weak signals at the ankle which did not allow instrumental registration yet permitted acoustic measurement of the low ankle pressures in three quarter of the cases. The ear apparently is an excellent "spectrum anallyzer" with a most favorable signal-to-noise ratio. In view of this finding studies reporting low systolic ankle pressure values for the determination of which the moment of visible recovery of the Doppler signal was used, should be considered with suspicion.

The conclusion can be that the impossibility to record a Doppler signal at a specific location indicates severe arterial obstruction, usually an occlusion, upstream from the monitoring site. In the case of unfavorable local recording conditions - e.g. extensive scarring or hematoma - this is less certain.

10.3.5. General remarks and conclusions on the use of the selected parameters in the diagnosis of arterial obstructions

Neither the differentiation between angiographically "normal" and "abnormal" legs nor the determination of the segmental distribution of arterial obstructions were the very subject of this study. Nevertheless, some conclusions 
about these topics can be drawn from the foregoing analyses.

In this study the presence of hemodynamically important obstructions in the arteries of the lower limb could quite reliably be established by means of palpation of the popliteal artery. $\mathbb{t}$ is unlikely that this result would also have been obtained if a group of investigators in stead of one and the same would have performed the examinations. The ankle pressure index at rest came out of the analyses as the more objective parameter to be used for the differentiation between "normal" and "abnormal" legs. This is in agreement with conclusions of others (section 5.5.1). The most discriminative qualitative signal parameter appeared to be the maximum reverse frequency of the Doppler signal of the common femoral artery (MAX REV(Fi). There is, however, no need to use this parameter for the distinction between "normal" and "abnormal" legs.

For the distinction between (1) legs without important angiographic obstructions, (2) legs with obstructions mainly confined to the aortoiliac region. (3) legs with their obstructions mainly distal to the groin and (4) legs with obstructions at both these levels, it was possible to formulate complicated classification rules. Up to 6 different classifying formulas would need to be combined to approach correct classification percentages of only 70 . The most important parameters to be used for such classifications would be the score of the strength of the femoral (PULS FEM) and the popliteal pulsation (PULS POP). the qualitative classification of the ankle signal (ANTHU), the maximum reverse frequency (MAX REV (F)) and the upslope time (UST(DER) (F)) both of the common femoral Doppler signal. This complicated method, for a great deal based on poorly reproducible and subjective parameters like the estimated pulse score and resulting in only low percentages of correct diagnosis, seems no asset to the clinician and consequently can not be recommended.

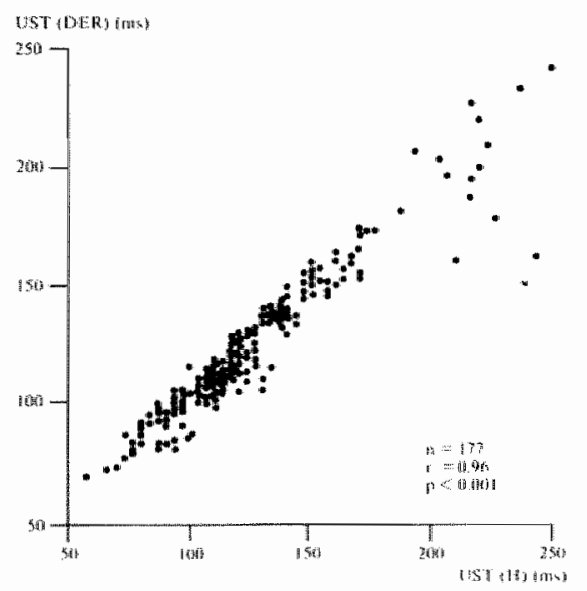

FIGURE 10.22

Correlation between UST $(H)$ and UST (DER) of the Doppler signal of the comm mon femoral artery.

The following parameters appeared to be valuable in recognizing hemodynamically important aortolliac obstructions by means of the described clinical and noninvasive Doppler examination:

- the score of the femoral pulse strength (PULS FEM).

- the thigh pressure index (TPI).

- the maximum reverse frequency of the femoral Doppler signal (MAX REV (F)). 
- the upslope time of the femoral Doppler signal determined by "hand" method (UST (H) (F)) or with the "derivative" method (UST (DER) (F)).

Between the UST (H) (F) and the UST (DER) (F) an excellent relationship was found for a wide range of values (Figure 10.22 ). It seems justified to use in classification procedures the very UST which is most easily obtained with the avallable equipment and substitute the UST (H) (F) for the UST (DER) (F) in the classification formulas and vice versa if such is more practical. The choice of the best classification rule was not possible on the ground of the obtainable diagnostic results, since these were quite similar for the various formulas (see Table 10.6). Neither did the analysis of false-positive and false-negative classifications give a clear indication about a possible superiority of one of the classifiers. On theoretical grounds (section 10.3.3.1) the parameters combined in the SET III classification formula (TPI and UST $(\mathrm{H})(\mathrm{F})$ ) will be least influenced by examiner bias or equipment operation. This classification formule, therefore, is preferred for the diagnosis of aortoiliac obstruction. 


\section{SUMMARIZING DISCUSSION OF RESULTS, RECOM- MENDATIONS AND CONCLUSIONS}

Patients with symptoms of obstructive arterial disease of the lower limbs may expect from their physicians an assessment of the pathophysiological state of the arterial system. During the last two decades a variety of so-called functional tests had come into clinical use for this purpose. The vascular surgeon has the specific additional task to establish the anatomical location of obstructions and their relative contribution to the total functional defict. The diagnosis of hemodynamically significant aortoiliac obstruction is an example of such a diagnostic problem. Because of its great practical importance in vascular surgery (section 1.1) it was selected as the subject of the present study.

The dilemma of this and similar studies is the fact that neither for the arterial system of the whole limb nor for the aortoiliac pathway in particular, a "yard stick" or standard exists to measure its "functional state". The term "functional state" or "functional capacity" is a mere abstraction. Probably the best method to define the hemodynamic significance of an aortoiliac obstruction, presently available is direct intraarterial measurement of the femoral artery pressure or of the aortolemoral pressure gradient (section 2.2.4, DARLING et al 1979). The method is not applicable on a wide scale and has until now mainly been used at the time of preoperative angiography. In the design of future studies on the value of noninvasive tests for the functional description of aortoiliac obstructions, serious consideration should be given to the inclusion of direct pressure measurements in the protocol.

Notwithstanding the term "functional" which is in vogue for the noninvasive arterial tests consisting of flow and/or pressure measurements and the observation of pulse waveform changes, in most recent studies the tests were ultimately employed to predict the anatomic extent and location of the obstructions as they appeared on angiograms (see references in section 1.2). Also in the present study functional tests were used to predict angiographic $(=$ anatomic) conditions of which might be assumed that they would have hemodynamic significance. Literature review led to the acceptance of a stenosis percentage of over 50 percent (diameter reduction) as a criterion of hemodynamic significance (section 3.5.2.2.).

In the light of the exactness suggested by measurable and calculable parameters of the circulation. like the ones used in this study, it was a surprise to see the traditional pulse palpation reach a good correlation with angiography (section 10.1.2) and emerge as the primary classifier in different classification procedures (sections 10.3.1, 10.3.2 and 10.3.3.1). This reminds one of an opening remark by the editor of a recent comprehensive textbook on vascular surgery: "There are few areas in medicine in which the conditions encountered lend themselves so readily to diagnosis solely on the basis of thoughtrul history and careful physical examination as do vascular diseases" (RUTHERFORD 1977, p. 3).

The segmental pressure indices in this study were in agreement with the findings previously reported in literature (Chapter 5). The ankle pressure indices at rest decreased with increasing severity of clinical disease (section 10.1.3). The ankle pressure index turned out to be a good criterion for the 
distinction between angiographically normal and abnormal limbs (section 10.3.1.). In the diagnosis of the segmental distribution of obstructions and the identification of aortoiliac obstruction it was of no value. This study confirmed the presently well recognized drawback of thigh pressure measurement if this is used to diagmose significant aortoiliac obstruction. Occlusions at the origin of the arteries branching off from the common femoral artery are an important cause of low thigh pressure readings (sections 5.5.3,5.5.4 and 10.1.3). This fact considerably reduces the specificity of thigh pressure measurement in the diagnosis of aortoiliac disease (section 8.5). In the present study this was evidenced by the high proportion of aortoiliac segments classified falsely positive for which this phenomenon probably could be held responsible (section 10.3.3.2). Nevertheless, the thigh pressure index proved to be one of the most important noninvasive parameters in the identification of significant aortoiliac obstruction (sections 10.3.3.1 and 10.3.5).

Ankle pressure determination in conjunction with stress testing on a treadmill was only of limited value. The walking test itself produced no useful objective parameters as also could have been anticipated from the literature review (sections 5.2 and 10.1.4). The response of the ankle pressure to the treadmill testing has some place in the differentiation between angiographically normal and abnormal legs. In this study a nearly normal response (type o or 1 ) was a quite certain indication of an arterial system without evident obstructions (section 10.1.5). The fact that the ankle pressure response type figured in the series of parameters which were useful for the distinction between "normal" and "abnormal" legs (Table 10.3) confirmed this. Unrecordable ankle pressure for some period after exercise might be taken as a relatively certain indication of the presence of multilevel arterial disease. Interpreted in this way this response might turn the scale if one would be in doubt about the significance of an angiographic lesion in the presence of a second lesion of proven significance. For the detection of aortoiliac obstruction the determination of the ankle pressure response to exercise was irrelevant.

The idea to use the damping factor of the aortoiliac segment (Chapter 7) as a parameter of arterial obstruction in that region had to be abandoned due to the high proportion of aortic Doppler signals which either could not be obtained at all or had to be rejected retrospectively because of poor quality (section 10.1.7.1). It was suggested that the fallure to obtain an aortic Doppler registration of acceptable quality under otherwise favorable recording circumstances fastens the suspicion on aortic pathology (section 10.1.7.1) athough too much remains uncertain about the cause of the phenomenon to use it as a diagnostic sign. It is imaginable that similar bad experiences with the recording of the aortic Doppler signals by other investigators are the explanation for the lack of scientific reports on Doppler waveform damping in the aortoilac segment (section 8.3).

A non-recordable Doppler signal at the common femoral or ankle level in the absence of local conditions which are known to interfere with a good Doppler examination, is a certain indication of severe upstream or local occlusion of the artery (section 10.3.4).

Measurement of the aorto-to-femoral pulse wave transit time - a laborious examination to perform and one which afterwards still involves much off-line processing and calculation - did not pay. In about one fourth of all legs the necessary simultaneous registration of an aortic and femoral signal of good quality could not be obtained due to the poor accessibility of the aorta to 
Doppler examination. The resulting values of the legs in which measurement was possible showed no correlation with the degree of aortoliac obstruction. This applied both to the observed and the normalized transit time values. The differences in transit time which were found between normal reference legs and apparently unobstructed legs of patients may be explained by a different vessel compliance. The transit time, which is a parameter of vessel wall stiffness rather than of degree of stenosis (section 7.1), was not useful for the assessment of aortoiliac obstruction.

The results of this study showed that the qualitative classification of the Doppler signals of the common femoral and the ankle arteries might be used as a means of approximately estimating the location of important obstructions. In the same way in which pulse palpation is used for that purpose, the degradation or worsening of the Doppler signal along a vascular segment can be taken as an indication of obstruction in that segment. A high degree of accuracy may not be expected of this method. The correlation between the femoral Doppler signal score and the degree of aortoiliac obstruction was poor. Only a femoral signal score of 3 in the classification according to Thulesius (section 6.2.2), which is characterized by the presence of a reverse flow component, can be taken as an indication that probably no significant aortoiliac obstruction will be found on the angiogram (section 10.1.8.2). In the final assessment of the relative importance of the various parameters for the identification of significant aortoiliac obstruction this qualitative criterion ranked only low.

A characteristic feature of all quantitative parameters of the Doppler signals used in the present study was their overlap of values for the various angiographic classes of limbs. Consequently, the selection of the most discriminative parameter was only possible by means of elaborate statistical analysis procedures. A notably exceptional position among the quantitative signal parameters was taken by the pulsatility index (PI). Scientific literature on Doppler waveform analysis of the last decade was replete with studies on the use of this theoretically elegant parameter. This was also the reason to discuss it quite extensively in this thesis (section 6.3.3). Recently the value of the PI in the assessment of aortolliac obstruction has repeatedly been relativized, especially in cases with multilevel obstructive disease. In the present study an important technical drawback of the PI come forward. Conditions which produce ambiguities in the maximum frequency envelope of the reverse flow component of the Doppler signal - for instance turbulence in the common femoral artery due to an iliac stenosis - introduced errors of great magnitude into the calculation of the PI (section 10,1.7.3). Very recently this source of error was also reported by HUMPHRIES and co-authors $(1980)$. In the present study quantitative analysis of the femoral Doppler signals was combined with an element of reactive hyperemia. A test was selected in which the signals at rest were compared to those one and three minutes after release of thigh occlusion. The design of this test was bases on the fact that obstructive arterial disease of a limb goes with a prolonged post-occlusion hyperemic reaction in that limb (section 9.2). It was assumed that the degree in which the values of "hyperemic" signal parameters would differ from the resting values, could contribute to a better differentiation between various categories of angiographic abnormalities. This did not appear to be true. The differences between signal parameter values at rest and three minutes after thigh occlusion were negligible and the parameters of the signals recorded one minute after thigh occlusion did not discriminate better than the parameters obtained at rest (section 10.2). The very 
hyperemia test used in the present study herewith turned out to have no diagnostic value. A pparently the moments of signal recording were not selected appropriately. The most marked effects of reactive hyperemia manifest themselves in the first minute of it. In the light of literature data (sections 2.2 .6 and 9.2$)$ and the resuits of this study, it seems recommendable to perform any measurement within the first minute to gain the maximum from such hyperemia tests (FRONEK et al 1973a, FRONEK et al 1977).

The two most discriminating qualitative Doppler signal parameters for the three diagnostic problems described in this study were the maximum reverse frequency of the femoral signal (MAX REV (F)) and the upslope or rise time of the femoral signal (UST (F)). Also in studies of the group of Fronek and Bernstein, San Diego, (section 6.3.1) the peak reverse flow velocity in the femoral and ankle artery was recognized as one of the best discriminating Doppler signal parameters together with the deceleration of the systolic downslope of the wave form (DILLEY and FRONEK 1978). When HUMPHRIES and co-workers (1980) replicated the studies of Goslings' laboratory on the use of the pulsatility index, the damping factor and the transit time for the differentiation between various degrees of obstruction in the femopopliteal pathway (GOSLING and KING 1974a), they additionally included eleven other Doppler waveform parameters in their analysis. That study could only partly confirm Goslings' findings on the value of damping factor and transit time for the quantification of femoropopliteal obstruction while at the same time showing that an equally sensitive diagnosis could be obtained from the simpler measurement of the rise time of the waveform (comparable to the UST of the present study). In a study by WATERS and co-workers (1977) good diagnostic results were reported of a so-called Proximal Damping Quotient (also discussed in section 7.2) which is a quantative expression of the upslope of the Doppler waveform. These studies all can be taken for a confirmation of the value of the two prominently figuring parameters of the present study, the MAX REV and the UST. A notable pro of these two parameters is the fact that both can readily be measured by hand without the use of calculators, planimetric devices or the like. The upslope time possibly has the additional advantage that its value measured from a sonagram will not differ substantially from its value determined from a zero-crossed signal record. If this could experimentally be confirmed it would open perspectives for the application of the parameter in simply equipped vascular laboratories.

The answer to the question which tests and examinations would practically be the most advisable ones for a noninvasive assessment of the aortoiliac pathway would best be tailored to the circumstances under which the diagnosis must be made. A clinician who only has the opportunity to perform routine clinical examination can have some confidence in femoral pulse palpation in the light of this study. If he also has a simple Doppler device (say a pocket apparatus) at his disposal he would be advised to measure a thigh pressure index and get used to listening to the character of the Doppler sounds of the common femoral artery. A clinician with a well equipped vascular laboratory at his disposal should ask that laboratory to determine the thigh pressure indices and provide (preferably sonagraphic) registrations of the femoral Doppler signals with measurements of the upslope times of the systolic waveforms. The thus gathered information certainly can not be considered a substitute for angiography. It rather will hellp to establis the true character of equivocal angiographic lesions in the aortoiliac region. If no angiography has been performed the ultrasound Doppler studies 
may be of help in the decision about the angiographic techniques to be chosen. Finally the tests may help to evaluate objectively the immediate effects of therapeutic interventions in the aortoiliac pathway and provide the parameters for follow-up studies.

In conclusion, the results of the present study allow the following answers to the questions put in the introduction to this thesis (section 1.2):

- The most valuable Doppler signal parameters for the diagnosis of the degree of aortoiliac obstruction (more or less than 50 percent diameter stenosis) are the maximum reverse frequency (MAX REV) and the upslope time (UST) of the systolic component of the signal recorded at the common femoral artery.

- This study does not answer the question of the value of Doppler signal analysis under conditions of hyperemia. It permits only the conclusion that the very hyperemia test employed in this study gives no diagnostic improvement above measurement at rest. Literature studies indicate that other hyperemia tests in combination with Doppler examinations are useful.

- Determination of the time delay between aortic and common femoral Doppler signals (transit time measurement) is of no use in the diagnosis of aortoilliac abstruction.

- In the comparison of the diagnostic value of Doppler waveform analysis to that of normal physical examination and of segmental systolic pressure measurement in the leg, all three are about equivalent if considered solely. If the relative contribution of each of the examinations to the diagnosis is considered in a combined use, the signal analysis occupies the third place.

- The most practical combination of Doppler lests which allows the best agreement with angiography in the diagnosis of aortoiliac obstruction is the determination of the thigh pressure index (TPI) and the upslope time of the femoral Doppler signal (UST(H) (F)). Both parameters are combined in the classification rulte $\mathrm{Q}=\mathrm{TPI}-0.003 \times \operatorname{UST}(\mathrm{H})(\mathrm{F})$.

The criterion for significant aortoiliac obstruction selected in this study was $Q \leqslant 0.57$. 


\section{SUMMARY}

A clinical study on the use of a variety of noninvasive ultrasonic Doppler tests in the diagnosis of chronic aortoiliac obstructive disease is described. It was performed in patients who also underwent aortography, the standard to which the test results were compared.

\section{CHAPTER I}

The aim of the study - described in this chapter - was to determine the practical value of Doppler tests for the diagnosis of aortoiliac obstruction, with emphasis on the value of waveform analysis of Doppler registrations. The study was designed in such a way that answers could be expected to questions regarding the most valuable signal parameters, the optimal anatomic site of signal recording, the use of Doppler registrations during hyperemia and the value of aorta-to-femoral pulse wave transit time measurements. Further problems to be solved by the study were: which is the relative value of Doppler signal analysis compared to both physical examination and segmental pressure measurements in the limbs and which is the optimal combination of tests?

\section{CHAPTER 2}

General aspects of chronic aortoiliac obstructive disease are outlined in this chapter. It deals with etiology and pathology, the different morphologic classifications in use and some figures on incidence and frequency distribution of the syndrome. Symptoms and signs are briefly discussed. Special attention is paid to the shortcomings of angiography for the diagnosis of aortoiliac obstructions, which are its underestimation of the severity of the lesions and the failure to provide functional information. The positive value of direct arterial pressure determination for the functional assessment of angiographically equivocal lesions is discussed. The chapter further contains general reviews on the analysis of plethysmographic pulse waveforms, blood low measurements and determinations of pulse wave transit times for the diagnosis of aortoiliac obstruction.

\section{CHAPTER 3}

Hemodynamic principles of arterial circulation are discussed. The existing close relationship between pulsatile arterial pressures and flow velocities is described. The origin of the flow velocity pulse wave and its changes along the peripheral arteries of the lower limb as well as the mechanisms which influence these changes are described. The meaning of flow velocity profiles is briefly mentioned. The reverse flow phase in the arterial circulation is extensively discussed because of its importance for the diagnosis of arterial pathology. The second part of the chapter deals with the pressure reducing influence of stenosis, which not only depends on the degree of the stenosis but also on the velocity of the flow through it. In this light the concept of "critical stenosis" is relativized. 
The influences of the degree, the length and the multiple character of stenoses on pressures and the related flow velocity waveforms are discussed. Also the influence of turbulence on the fall in pressure is discussed. The chapter ends with a short discussion about the hemodynamic effects of arterial aneurysms.

\section{CHAPTER \&}

This chapter deals with the application of the Doppler effect in flow velocity detection. Specific pros and cons of continuous wave and pulsed Doppler systems and the methods to detect flow direction are discussed from a mainly technical point of view. The factors which determine the range or spectrum of frequencies obtained in Doppler examinations of blood vessels are discussed. They are the instantaneous velocity profile of the blood stream in the vessel, the characteristics of the instrument employed and the way in which the ultrasound beam intersects the blood vessel. The three most important modes of Doppler signal processing - acoustic amplification, zero crossing detection (ZCD) and audio frequency spectrum analysis - are described and their adwantages and drawbacks are discussed.

\section{CHAPTER 5}

This chapter reviews the literature on segmental systolic pressure measurements in the lower limb. It deals with methods of measurement at various levels of the leg. Results of thigh and ankle pressure readings both at rest and under hyperemic conditions in normal persons and in patients with different stages of oblite rative arterial disease and a va riety of angiographic obstructive lesions are summarized. The measured pressure values depend on the severity of the underlying disease, the widths of the sphygmomanometer cuffs used and the functional state of the circulation. It is concluded from the literature data that the ankle pressure values correlate with the severity of arterial obstructions in general but do not allow an anatomic diagnosis of the level of obstruction. The lafter can reasonably well be obtained by segmental pressure readings at various levels of the leg. Another conclusion is that lowered thigh pressures can be used as an indication of a ortoiliac obstruction but that the diagnostic specificity of this measurement is reduced by the fact that also obstructions in the arteries of the upper leg may cause a lower reading of the thigh pressure.

\section{CHAPTER 6}

Qualitative and quantitative ways of analyzing the Doppler signal and the clinical value of various classifications of the signal and of parameters derived from its waveform registration, are discussed in this chapter. A qualitative assessment of the signal can be performed either acoustically or by visual classification of the waveform pattern. The application of Thulesius" pattern classification is discussed. It is concluded that the described qualitative signal analysis methods are useful additions to routine physical examination of vascular patients, mainly to improve the presumptive diagnosis of the segmental distribution of obstructions in the limb. Quantitative signal parameters are waveform amplitudes (maximum and mean, forward and reverse), time intervals of the waveform (upstroke and downstroke time of the systolic wave) or derivatives of these basic measurements (amplitude ratios, acceleration, 
deceleration). The conclusions drawn from the scarcely reported studies on the clinical application of these signal parameters are that amplitude parameters have only limited value in the evaluation of vascular disease and that the time domain parameters seem to contain information about the condition of the inflow and the outflow vessels of the artery from which the Doppler signal was derived. However, exact data on this last aspect are still lacking. An extensive discussion is devoted to the pulsatility index (PI) of Doppler signals which received much attention in the literature during the last decade. It is concluded that the PI depends on the severity of upstream arterial stenosis but is also influenced by the state of the distal vascular bed. The PI of the Doppler signal of the common femoral artery has a definite relation to the severity of aortoiliac obstruction. Its value in the assessment of aortoiliac obstruction in multilevel disease has recently repeatedly been relativized.

\section{CHAPTER 7}

This chapter describes the use of the segmental Doppler parameters "pulse wave transit time" and "damping factor". Normal values for both parameters are given for the different vascular segments of the lower limb. Until now most studies in which the segmental parameters were used either solely or in combination, only dealt with the femoropopliteal pathway. Applications of the two parameters are described. No clinical applications in the aortoiliac pathway are reported.

\section{CHAPTER 8}

For the various Doppler examinations described in the preceding chapters, in this chapter an attempt is made to compare their value as a means of diagnosing aortoiliac stenosis of more than 50 percent diameter reduction. To this purpose the diagnostic accuracy, specificity and sensitivity which could be obtained with each method, as they could be calculated retrospectively from the published data, were compared to each other. Only few publications do contain the information to do so. For thigh pressure measurements comparable figures became available which indicate that the specificity of the measurement can be improved by combining upper thigh measurements with more distal readings or by the use of narrow sphygmomanometer cuffs. For qualitative and quantitative Doppler signal parameters exact figures are lacking. The importance of the combination of thigh pressure readings with femoral Doppler signal analysis for the diagnosis of atortoiliac obstruction is emphasized.

\section{CHAPTER 9}

The examinations and tests, angiographic technique, specifications of instruments and the principles of the statistical analyses of obtained data employed in the present study, are described and explained in this chapter. The studied population was a group of 96 patients who underwent aortography, 78 men (age $60.9 \pm 9.9$ years) and 18 women (age $57.5 \pm 15.4$ years) and a group of 28 normal reference subjects who were not angiographed. The basic material of the study comprises angiographic data, results of physical examinations and of an exercise test, systolic pressure readings at the limb and the outcome of the analysis of Doppler signal recordings. 
Angiograms are characterized by two figures or scores, one for the proximal section including the common femoral artery and the upstream arteries ("inflow") and one for the arteries of the section below the common femoral artery ("outflow"). Both scores are a function of the degree of arterial stenoses in the angiographic sections and can range from zero, for a normal angiogram, 10100 for a section with occlusions. Based on whether the two angiographic scores are $\leqslant 50$ or $>50$ each limb is assigned to one of four possible a priori angiographic classes. Legs with inflow scores $\leqslant 50$ are considered to be free of significant aortoiliac obstruction whereas limbs with inflow scores $>50$ are classified in the group of legs with aortoliac obstruction. Physical examination includes grading of the strength of femoral, popliteal and ankle pulsations at rest in a 4-points scoring system. Exercise consists of a standardized treadmill walking test. Systolic pressures are determined (Doppler method) under resting conditions at the thigh and the ankle and during a 15 minutes period after the treadmill test at the ankle. The values are expressed as the ratio of the almost simultaneously recorded brachial artery pressure. The ankle pressure responses to exercise are classified in a 6-classes system. With continuous wave devices Doppler signals are recorded from the abdominal aorta, the common femoral arteries and the ankle arteries at rest and also from the common femoral arteries one and three minutes after thigh occlusion, used to induce hyperemia. For the determination of the pulse wave transit time the abdominal and femoral signals are recorded simultaneously as well. The off-line analysis of the Doppler signals is performed on sonagrams of the signals which are obtained through frequency spectrum analysis of the audio signals. The maximum frequency envelope of the sonagram is either classified qualitatively in a 4-points scoring system (Thulesius) or its digitized waveform is analyzed by means of a computer which calculates 9 numerate parameters. These signal parameters are: the maximum forward frequency, the mean forward frequency, the maximum reverse frequency, the pulsatility index, the ratio of maximum reverse/maximum forward frequency, the systolic upslope time (hand measurement), the systolic upslope time (derivative measurement), the systolic downslope time and the socalled null fraction.

The chapter ends with a description of the statistical procedures used for the selection of the most discriminative noninvasive test parameters and for the formulation of classification rules. A discussion on the Receiver Operator Characteristic ( $R O C$ ) analysis used for the selection of optimal classification thresholds in a two-classes problem closes the chapter.

\section{CHAPTER 10}

Three major parts can be distinguished in this chapter which contains the results of the study.

In the first part the mean values and standard deviations for the different scores, test results, signal and segmental parameters, obtained in this study, are summarized for the normal legs and for the patients" legs classified in the four angiographic a priori classes. Concise comments on these results are included in this chapter. A main characteristic of all parameters is a considerable overlap of the values for the different angiographic classes and normals. For the femoral pulse score, the thigh pressure index, the qualitative score of the femoral Doppler signal and the observed as well as the normalized aorto-femoral transit times, also the correlation between the angiographic inflow scores and the 
values of the parameters is presented. This relationship is good for the pulse strength, reasonable for the thigh pressure index, poor for the qualitative signal score and non-existing for the transit times. In this part atso the quality of the sonagraphic Doppler registrations and possible artifacts are discussed. In 38.7 percent of all cases (and in 44.8 percent of the patients) aortic Doppler signal analysis was impossible for various reasons which are discussed. Due to this neither the calculation of a damping factor was possible.

In the second part of the chapter a preselection of test parameters before the final analyses is discussed. Transit time measurements, post-occlusion femoral Doppler signal parameters and the pulsatility index are excluded from further analyses since they have no or very little pertinence to the classification of legs.

Four sets of remaining test parameters are described of which the diagnostic value is further analyzed in the third part of the chapter. These parameter sets are rated to their value for the discrimination between angiographically "normal" and "abnormal" legs, for the segmental diagnosis of arterial obstructions and for the diagnosis of aortoiliac obstruction. The discrimination "normal/abnormal" is most objectively performed by means of ankle pressure determinations. The classification of legs in four different angiographic classes, distinguishing between obstructions mainly located in the inflow section or in the outflow section or in none of these or in both, is only very imperfectly possible and demands very complicated and unpractical classification procedures. For the detection of significant aortolliac obstruction the following parameters are found to be important: the femoral pulse strength, the thigh pressure index, the maximum reverse frequency and the upslope time of the femoral Doppler signal at rest. On grounds which are discussed in the chapter, a classification formula, incorporating the thigh pressure index and the upstope time is preferred. With this method a diagnostic accuracy of 84 percent, a specificity of 84 percent and a sensitivity of 83 percent could be obtained in the detection of significant aortoiliac obstruction.

\section{CHAPTER II}

This last chapter contains a summarizing discussion of the final results and some recommendations for future studies and the application of noninvasive tests in daily clinical practice. The conclusions of this study regarding the diagnosis of aortoiliac obstruction are:

- the most useful signal parameters are the maximum reverse frequency and the duration of the systolic upslope of the femoral Doppler registration.

- no judgment is possible on the value of signal registration under conditions of hyperemia since the very hyperemia test employed in this study was not appropriate.

- aorto-femoral transit time measurement is not useful.

- clinical examination (= pulse palpation), segmental systolic pressure measurement and Doppler signal analysis are all eclually valuable.

- a practical combination of Doppler parameters is that of the thigh pressure index with the upslope time of the femoral signal combined in aclassification rule. 
Er wordt een studie beschreven naar het nut van verscheidene niet invasieve ultrasone Doppler onderzoekingen ten behoeve van de diagnostiek van chronische vaatvernauwingen in het aortoiliacale traject. De studie werd verricht bij patienten die tevens aortografie ondergingen. De resultaten van de verschillende testen werden vergeleken met de uitkomsten van de angiografie.

\section{HOOFDSTUK 』}

Het in dit hoofdstuk beschreven doel van de studie is het vaststellen van de praktische waarde van Doppler onderzoekingen voor het diagnostiseren van aortoiliacale obstructies. De nadruk ligt hierbij op de betekenis van de analyse van geregistreerde Doppler kurven. De opzet van de studie is een antwoord te vinden op vragen betreffende de voor dit doel meest geschikte anatomische plaats voor de Doppler registraties, de meest bruikbare parameters van het signaal, het nut van signaal registratie tijdens een hyperaemische fase van de circulatie en de waarde van het bepalen van de looptijd van de polsgolf tussen aorta en a.femoralis communis. Tevens is het de bedoeling de waarde van signaalanalyse te vergelijken zowel met die van het normale lichamelijke onderzoek als met die van segmentele bloeddrukmetingen aan de benen en de vraag naar de optimale combinatie van testen te beantwoorden.

\section{HOOFDSTUK 2}

In dit hoofdstuk wordt een algemeen overzicht gegeven van het chronisch obstruerende aortoiliacale vaatlijden. Achtereenvolgens worden vermeld de definitie, de oorzaken, patholoog-anatomische aspecten, enkele gebruikelijke indelingen van het anatomische patroon van de afwijkingen en epidemiologische gegevens van het ziektebeeld. Anamnestische en fysisch diagnostische bevindingen bij dit ziektebeeld worden kort behandeld. Speciale aandacht wordt besteed aan de tekortkomingen van de angiografie als diagnosticum van stenoserende aortoiliacale afwijkingen. Bij angiografie wordt vaak de ernst van de vernauwingen onderschat en er wordt geen funktionele informatie verkregen. De waarde van bloedige arteriele druk metingen voor het vaststellen van het eventuele funktionele belang van twijfelachtige angiografische afwijkingen in het aortoiliacale traject wordt besproken. Verder bevat dit hoofdstuk literatuuroverzichten over analyse van plethysmografisch verkregen kurven. meting van de bloedstroomsterkte ("flow") en bepalingen van de looptijd van de polsgolf in het kader van de diagnostiek van aortoiliacale vernauwingen.

\section{HOOFDSTUK 3}

Hierin worden de haemodynamische principes besproken van de arteriele circulatie. Aandacht wordt besteed aan de nauwe relatie tussen de, door de hartslag bepaalde, arteriele drukschommelingen en de wisselende stroomsnelheden. Het ontstaan van de stroomsnelheidskurve en de vormveranderingen die 
deze kurve ondergaat op har weg langs het arteriele systeem, alsmede de mechanismen die verantwoordelijk zijn voor deze veranderingen, worden vermeld. De betekenis van stroomsnetheidsprofielen wordt kort besproken. Veel aandacht wordt besteed aan de terugstroom- of afstroomfase ("reverse (low") in de arteriele circulatie vanwege het belang dat het vaststellen van stroomomkering heeft woor de diagnostiek. Het tweede deel van het hoofdstuk behandelt de drukverlagende invloed van de stenoses. Deze wordt niet alleen door de stenosegraad bepaald, maar ook door de bloedstroomsnelheid door de stenoses heen. In dit kader wordt het begrip "kritische stenose" gerelativeerd. De invloed van de mate van de vernauwing, van de lengte van de stenose alsmede van het effect van meerdere stenoses achter elkaar op het drukverval over de vernauwing(en) en de daardoor bepaalde stroomsnelheidskurven wordt besproken. Tevens wordt melding gemaakt van de invloed die turbulentie op de druk heeft. Het hoofdstuk eindigt met een korte discussie over het haemodynamische effect van aneurysmata.

\section{HOOFDSTUK 4}

In dit hoofdstuk worden de toepassingen van het Doppler effect bij de bepaling. van bloedstroomsnelheden, de specifieke voor- en nadelen van continu uitzendende ("continuous wave") en intermitterend uitzendende ("pulsed") Doppler systemen alsmede de voorzieningen om de richting van de bloedstroom vast te kunnen stellen vanuit een voornamelijk technisch standpunt besproken. De verschillende factoren die het, bij Doppler onderzoekingen verkregen, frequentie spectrum bepalen passeren de revue. Deze zijn: het telkens wisselende snelheidsprofiel van de bloedstroom ter plaatse van de meting, bepaalde karakteristieken van de gebruikte apparatuur en de wijze waarop de ultrageluidsbundel het bloedvat doorkruist. De drie belangrijkste manieren om het Doppler signaal te verwerken - akoestische versterking, verwerking door middel van een nuldoorgangen teller ("zero-crossing detection") en spectraal analyse - worden besproken en hun voor- en nadelen worden vermeld.

\section{HOOFDSTUK 5}

Dit hoofdstuk geeft een literatuur overzicht over segmentele bloeddrukmetingen aan de benen. De methodiek van deze metingen op verschillende niveaux van het been wordt besproken. Er wordt een overzicht gegeven van de meetresultaten zowel onder rustomstandigheden als tijdens hyperaemie bij normalen en bij patienten met verschillende stadia van vaatlijden en voor verscheidene angiografische afwijkingen. Het meetresultaat hangt af van de ernst van de vataandoening, de breedte van de gebruikte bloeddrukmanchetten en de toestand warin de circulatie zich bevindt, namelijk in rust of in een lase van versnelde bloedstroom. Uit de literatuur wordt de conclusie getrokken dat de enkeldrukmetingen correleren met de algemene ernst van de vaatvernauwingen, maar geen anatomische informatie geven over de plaats van die vernauwingen. Deze laatste informatie kan wel worden verkregen uit gecombineerde metingen op meerdere niveaux van het been. Een conclusie betreffende de dijdrukmetingen is dat deze, indien verlaagd, een aanwijcing kunnen geven over het bestaan van aortoiliacale vaatvernawwingen. De diagnostische specificiteit van deze bepalingen wordt echter verminderd door het feit dat ook obstructies in de bovenbeensarteriën de oorzaak van verlaagde dijdrukmeting 


\section{HOOFDSTUK 6}

Kwalitatieve en $k$ wantitative methoden van analyse van het Doppler signaal en de klinische waarde van verschillende klassificaties van het gehele signaal of van afgeleide signaal parameters worden in dit hoofdstuk besproken. Een $k$ walitatieve beoordeling van het Doppler signaal kan zowel akoestisch plaatsvinden als visueel aan de hand van een grafische registratie van het signaal. Besproken wordt de patroon klassificatie volgens Thulesius. Aangaande de beschreven kwalitatieve signaal analyse wordt geconcludeerd dat deze een waardevolle aanvulling is op het gebruikelijke lichamelijke onderzoek, vooral voor het vaststellen van het niveau waarop vernawwingen in de beenvaten voorkomen. Kwantitatieve parameters van een Doppler kurve kunnen zijn: amplitude- of hoogtematen (maxima of gemiddelden van het toestroom- of afstroomsignaal), tijdsintervallen (stijgtijd en daaltijd van de systolische component van de kurve) of afgeleiden van deze basale metingen (verhoudingen tussen amplitudo's, versnelling, vertraging). De conclusies die uit de schaarse publicaties over het gebruik van dit soort signaal parameters kunnen worden getrokken, zijn dat amplitudeparameters slechts een beperkte waarde hebben bij de beoordeling van vaatafwijkingen, maar dat de tijdsintervallen informatie lijken te bevatten over de toestand van het vaatstelsel proximaal en distaal van de plaats waar het Doppler signaal werd geregistreerd. Getalsmatige gegevens hierover ontbreken echter nog. Een uiltgebreide bespreking wordt gewijd aan de pulsatiliteitsindex (PI) ("pulsatility index") van Doppler signalen die de laatste jaren veel aandacht kreeg in de literatuur. De conclusie is dat de PI bepaald wordt door de ernst van stenosen stroomopwaarts, maar tevens wordt beinvloed door de toestand van het distale vaatbed. De PI van het Doppler signaal van de a.femoralis communis staat duidelijk in relatie tot de ernst van aortoiliacale obstructies. De laatste tijd is de waarde van de PI voor de beoordeling van aortoiliacale vernauwingen, bij het tevens voorkomen van afwijkingen op andere niveaux, herhaaldelijk ter discussie gesteld.

\section{HOOFDSTUK 7}

Dit hoofdstuk beschrijft het gebruik van de segmentele Doppler parameters. looptijd van de polsgolf ("transit time") en demping van de polsgolf ("damping factor"). Normale waarden voor beide worden vermeld voor de verschillende segmenten van de onderste extremiteit. De meeste studies warin deze beide parameters - hetzij als enige, hetzij in combinatie met elkaar - tot nutoe werden gebruikt, hadden allen betrekking op het femoropopliteale traject. Toepassingen van de beide parameters worden beschreven. Klinische toepassingen ervan in het aortoiliacale traject zijn door anderen niet beschreven.

\section{HOOFDSTUK 8}

In dit hoofdstuk wordt getracht de waarde van de verschillende, in de voorafgaande hooldstukken beschreven, Doppler onderzoekingen ten aanzien van de diagnostiek van aortoiliacale vernauwingen wan meer dan 50 procent diameter vermindering, met elkaar te vergelijken. Hiertoe werden, woor zover mogelijk, de diagnostische nauwkeurigheid, specificiteit en gevoeligheid ("ac- 
curacy, specificity, sensitivity") voor de verschillende onderzoekingen, berekend uil de in de publicaties voorkomende gegevens, met elkaar vergeleken. Slechts enkele publicaties bevatten de benodigde informatie. Voor de dijdrukmetingen werden zodoende vergelijkbare uitkomsten verkregen die laten zien dat de specificiteit wan deze bepaling kan worden verbeterd door metingen hoog aan de dij te combineren met meer distale metingen of door het gebruik van smalle bloeddrukmanchetten. Ten aanzien van $k$ wallitieve en kwantitatieve signaal parameters en de segmentele Doppler parameters ontbreken objectieve gegevens. Het belang van de combinatie van dijdrukmeting en van analyses van het Doppler signaal van de arteria femoralis wordt onderstreept.

\section{HOOFDSTUK 9}

De onderzoekingen en testen, de angiografie techniek, de specificaties van de apparatuur en de statistische methoden gebruikt bij de analyse van de verzamelde gegevens van het eigen onderzoek worden in dit hoofdstuk beschreven en toegelicht. De onderzochte populatie betrof 96 patienten die angiografie ondergingen, 78 mannen (leeftijd $60.9 \pm 9.9$ jaren) en 18 vrouwen (leeftijd $57.5 \pm 15.4$ jaren), en 28 normale proefpersonen die geen angiografie ondergingen. Het basis materiaal voor de studie omvat anglografiegegevens, de uitkomsten van lichamelijk onderzoek en van een inspanningstest, systolische drukwaarden gemeten aan de benen en de resultaten van de analyses van de geregistreerde Doppler signalen.

Ieder angiogram wordt gekenmerkt door twee getallen of scores, één voor het proximale gedeelte dat de a.femoralis communis omvat en de stroomopwaarts ervan gelegen arteriën ("inflow") en één voor de arteriën van het gedeelte stroomafwaarts van de a.femoralis communis ("outflow"). Beide scores worden bepaald cloor de mate van stenosering in het betreffende gedeelte van het angiogram en kunnen varieren tussen nul, voor een normaal angiogram, en 100 , voor een angiograffe gedeelte met totale afsluitingen. Afhankelijk van het feit of de angiografische scores $\leqslant 50$ of $>50 \mathrm{zijn}$, wordt ieder been toegewezen aan één van vier mogelijke apriori angiografische klassen. Benen met een "inflow" score $\leqslant 50$ worden in deze studie beschouwd vrij te zijn van belangrijke aortoiliacale obstructies, terwijl extremiteiten met "inflow" scores $>50$ aan de groep var benen met aortoiliacale obstructies worden toegewezen. Het lichamelijk onderzoek omvat het waarderen van de kracht van cle lies-, knie-en enkelpulsaties in een score systeem met vier mogelijkheden. De inspanning bestaat uit een gestandaardiseerde looptest op een tredmolen. Systolische bloeddrukken worden bepaald (Doppler methode) aan de dij en de enkel onder rustomstandigheden, alsook gedurende een periode wan 15 minuten na de looptest aan de enkel. Deze bloeddrukken worden weer gegeven als indices ten opzichte van de nagenoeg gelijktijdig aan de arm bepaalde bloeddruk. Het effect van de inspanning op de enkeldrukken wordt geklassificeerd in 6 verschillende typen. Met "continuous wave" apparatur worden in rust Doppler signalen geregistreerd van de aorta abdominalis, de a.femoralis communis en de arterien aan de enkel alsook aan de femoraalarteriën, één en drie minuten na een afsluiting van de circulatie in het been ter verkrijging van hyperaemie. Voor het bepalen van de looptijd van de polsgolf worden het aorta- en het femoralissignaal ook gelijktijdig vastgelegd. De analyse van de Doppler signalen wordt separaat uitgevoerd aan de hand van de sonagrammen die via spectrum analyse worden verkregen uit de audiosignalen. De vorm van de omhullende van de 
maximale frequenties op het sonagram wordt ofwel $\mathrm{kwaltatief}$ beoordeeld en geklassificeerd volgens een 4-punts score systeem (Thulesius) of in digitale worm toegevoerd aan een computer die er 9 numerieke parameters aan berekent. Deze signaalparameters zijn: de maximale frequentie van het toestroomsignaal, de maximale frequentie van het afstroomsignaal, de gemiddelde frequentie van het toestroomsignaal, de verhouding tussen de maximale afstroom/maximale toestroomfrequentie, de pulsatiliteitsindex, de systolische stijgtijd (handmeting), de systolische stijgtijd (meting met behulp van de eerste afgeleide), de systolische daaltijd en een zogenaamde nulfractie. Tenslotte volgt een beschrijving van de statistische methodieken die werden gebruikt voor het kiezen van de parameters met het beste onderscheidende vermogen en voor het formuleren van $k$ lassificatieregels. Het hoofdstuk wordt afgesloten met een bespreking van de "Receiver Operator Characteristic" (ROC) analyse die wordt gebruikt voor" het bepalen van optimaal scheidende criteria voor twee-klassen problemen.

\section{HOOFDSTUK 10}

Dit hoofdstuk, dat de resultaten van de studie bevat, valt uiteen in drie grote onderdelen.

Het eerste deel geeft een opsomming van de gemiddelde waarden met hun standaard deviaties van de verschillende scores, de testresultaten en de signaalen segmentele parameters die in de studie werden verzameld; alle gegroepeerd naar angiografïsche klasse van de benen. Deze resultaten worden kort becommentarieerd. Een belangrijk kenmerk is de overlapping van de warden van alle parameters in de verschillende angiografische klassen alsmede tussen deze klassen en de normalen. Daarnaast wordt voor de scores van de liespulsaties, de dijdrukindices, de kwalitatieve scores van het Dopplersignaal van de a.femoralis communis en voor de "gemeten" en de "genormalizeende" looptijden van de polsgolf de correlatie gegeven tussen de angiografische "inflow" scores en de parameterwaarden. De correlatie blijkt goed te zijn voor de pulsatiescore, redelijk voor de dijdrukindex, slecht voor de kwalitatieve signaal score en afwezig woor de beide looptijden van de polsgolf. Ook worden in dit gedeelte de kwaliteit van de sonagrammen en de voorkomende artefacten besproken. Analyse van het Dopplersignaal van de aorta was on mogelijk in 38.7 procent van alle gevallen (en in 44.8 procent der patienten). De verschillende oorzaken van dit verschijnsel worden besproken. Ten gevolge van de onmogelijk heid het aortasignaal te registreren wordt ook de bepaling van de demping van de polsgolf over het aortoiliacale traject onmogelijk.

In het tweede gedeelte van het hoofdstuk wordt een selectie van de parameters vóór de eindanalyses besproken. Looptijdmetingen van de polsgolf, parameters van het Dopplersignaal na occlusie van het been en de pulsatiliteitsindices worden van verdere analyse uitgesloten vanwege hun geringe betekenis voor de klassificatie der benen.

Vier series van overblijvende parameters worden beschreven die in het derde deel van het hoofdstuk worden onderzocht op de waarde die zij hebben voor het onderscheid tussen angiografifsch "normale" en "abnormale" benen, voor de segmentele diagnostiek van de vaatobstructies en voor de diagnose "artoiliacale obstructie". Het onderscheid "normaal/abnormaal" kan het meest objectief worden verkregen met behulp van enkeldrukmetingen. Het indelen van benen in vier verschillende angiografische klassen - onderscheiden all naar gelang de obstructies voornamelijk in het "inflow" gedeelte, het "outflow" 
gedeelte, in geen van beide of in beide gedeelten zijn gelocaliseerd - is slechts zeer gebrekkig mogelijk en vereist gecompliceerde en onpraktische klassificatieprocedures. Voor het herkennen van belangrijke aortoiliacale vernauwingen blijken de volgende parameters van belang: de kracht van de liespulsatie, de dijdrukindex, de maxmale frequentie van de afstroomcomponent en de stijgtijd van het Dopplersignaal wan de a.femoralis communis. Op grond van in het hoofdstuk gegeven overwegingen, wordt de voorkeur gegeven aan een klassificatieformule waarin de dijdrukindex en de stijgtijd voorkomen. Met deze methode kon een diagnostische nau wkeurigheid van 84 procent, een specificiteit van 84 procent en een gevoeligheid van 83 procent worden bereikt bij de diagnostiek van aortoiliacale obstructies.

\section{HOOFDSTUK $\|$}

Dit laatste hoofdstuk bevat een samenvattende discussie van de eindresultaten en enige a anbevelingen voor toekomstige studies alsmede aanbevelingen woor de toepassing van niet invasieve Doppler testen in de dagelijkse klinische praktijk.

De conclusies van de hier beschreven studie die betrekking hebben op de diagnostiek van aortoiliacale vernauwingen zijn:

- de meest waardevolle Doppler signaalpa rameters zijn de maximale frequentie van het terugstroomsignaal ("reverse") en de systolische stijgtijd gemeten aan het signaal van de a.femoralis communis.

- een oordeel over de waarde van signaal beoordeling onder hyperaemie omstandigheden is niet mogelijk, omdat de voor dit onderzoek gekozen hyperaemie test niet geschikt was.

- bepaling van de aorto-femorale looptijd van de polsgolf is van geen waarde.

- klinisch onderzoek (= polspalpatie), indirecte systolische dijdrukmeting en analyse van het femorale Dopplersignaal hebben ongeveer gelijke waarde.

- een praktische combinatie van Doppler parameters is die van de dijdrukindex met de stijgtijd wan het femorale signaal, gecombineerd in een klassificatieregel. 


\section{APPENDIX}

TABLE A-1

Numbers of patients" angiograms with their different combinations of inflow and outfow seores.

\begin{tabular}{crrrrrrrrrr}
\hline \multicolumn{10}{c}{ OUTFLOW SCORE } \\
& 0 & $1-25$ & $26-50$ & $51-75$ & $76-99$ & 100 & unknown & Total \\
\hline INFLOW SCORE & 0 & 22 & 5 & 4 & 1 & 1 & 0 & 6 & 39 \\
& $1-25$ & 13 & 8 & 4 & 2 & 3 & 0 & 0 & 30 \\
$26-50$ & 11 & 11 & 18 & 4 & 4 & 4 & 0 & 52 \\
& $51-75$ & 11 & 0 & 2 & 0 & 3 & 1 & 0 & 17 \\
$76-99$ & 5 & 2 & 2 & 0 & 1 & 1 & 0 & 11 \\
100 & 10 & 3 & 3 & 1 & 1 & 8 & 2 & 28 \\
& unknown & 6 & 1 & 1 & 0 & 0 & 1 & 6 & 1.5 \\
& Total & 78 & 30 & 34 & 8 & 13 & 15 & 14 & 192 \\
\hline
\end{tabular}

TABLE A-2.

Numbers of angiograms in the various angiographic classes with their mean inflow and outhow scores $( \pm \mathrm{SD})$.

\begin{tabular}{ccccc}
\hline ANGIOCLASS & $\begin{array}{c}\mathrm{N} \\
\text { legs }\end{array}$ & INFLOW SCORE & $\begin{array}{c}\text { OUTMLOW SCORE } \\
\text { mean } \pm \text { SD }\end{array}$ & SD \\
\hline 1 & 96 & 50.0 & $20.6 \pm 17.4$ & $17.1 \pm 20.2$ \\
2 & 19 & 9.9 & $27.7 \pm 1.9$ & $79.2 \pm 14.1$ \\
3 & 38 & 19.8 & $84.2 \pm 15.4$ & $10.3 \pm 18.5$ \\
4 & 16 & 8.3 & $87.2 \pm 12.2$ & $91.9 \pm 12.2$ \\
5 & 23 & 12.0 & & \\
\hline
\end{tabular}

TABLE A-3.

Angiographic classifications (angioclass) of all legs of the different patient categories (symproms).

\begin{tabular}{|c|c|c|c|c|c|c|c|c|}
\hline \multirow[t]{2}{*}{ SYMPTOMS } & & \multicolumn{2}{|c|}{ TOTAL (logs) } & \multicolumn{2}{|c|}{ ANGIOCLASS } & \multirow[b]{2}{*}{3} & \multirow[b]{2}{*}{ a } & \multirow[b]{2}{*}{5} \\
\hline & & $\mathbb{N}$ & $y_{2}$ & 1 & 2 & & & \\
\hline \multirow[t]{2}{*}{ Asymptomatic } & $n$ & 26 & & 18 & 0 & 0 & 0 & 6 \\
\hline & \% & & 100.0 & 69.2 & 0.0 & 7.7 & 0.0 & 2.7 .1 \\
\hline \multirow[t]{2}{*}{ Clatudication } & $n$ & 124 & & 59 & 14 & 34 & 5 & 12 \\
\hline & \% & & 100.0 & 47.6 & 11.3 & 27.4 & 40 & 9.7 \\
\hline \multirow[t]{2}{*}{ Rest pain } & $\mathrm{n}$ & 30 & & 13 & 5 & 0 & 9 & 3 \\
\hline & $\%$ & & 100.0 & 43.3 & 16.7 & 0.0 & 300 & 10,0 \\
\hline \multirow[t]{2}{*}{ Necrosis } & $n$ & 12 & & 6 & 0 & 2 & 2 & 2 \\
\hline & wh & & 100.0 & 50.0 & 0.0 & 16.7 & 16.7 & 16.7 \\
\hline \multirow[t]{2}{*}{ TOTAL } & $n$ & 192 & & 96 & 19 & 38 & 16 & 23 \\
\hline & $\%$ & & 100.0 & 50.0 & 9.9 & 19.8 & $B .3$ & 12.0 \\
\hline
\end{tabular}


TABLE A-4.

Numbers of legs with different pulsation scores at the femoral, popliteal and ankle level by angiographic classification of the legs (angioclass).

\begin{tabular}{clrrrr}
\hline ANGIOCLASS & PuLSATION SCORE & 3 & 2 & 1 & 0 \\
\hline 0 & femoral & 56 & 0 & 0 & 0 \\
& popliteal & 52 & 2 & 0 & 2 \\
& ankle & 54 & 2 & 0 & 0 \\
1 & femoral & 75 & 19 & 2 & 0 \\
& popliteal & 56 & 16 & 5 & 19 \\
& ankle & 68 & 4 & 5 & 19 \\
2 & femoral & 13 & 4 & 2 & 0 \\
& popliteal & 2 & 3 & 0 & 14 \\
& ankle & 3 & 1 & 2 & 13 \\
3 & femoral & 4 & 11 & 10 & 13 \\
& popliteal & 3 & 4 & 5 & 26 \\
& ankle & 5 & 5 & 3 & 25 \\
& femorall & 3 & 1 & 3 & 9 \\
& popliteal & 0 & 0 & 0 & 16 \\
& ankle & 0 & 0 & 0 & 16 \\
\hline
\end{tabular}

TABLE A-5.

Numbers and mean anglographic inflow scores ( \pm SD) of patients'legs with different combinations of femoral pulsation scores and presence or absence of pelvic bruits.

\begin{tabular}{|c|c|c|c|}
\hline FEMORAL PULSATION & PELVIC BRUTT & $\stackrel{N}{N}$ & $\begin{array}{c}\text { INFLOW } \$ C O R E \\
\text { mean } \pm \mathrm{SD}\end{array}$ \\
\hline 3 & - & 35 & $8.0 \pm 13.5$ \\
\hline 3 & t & 6.5 & $29.4 \pm 31.4$ \\
\hline 3 & $-o r+$ & 100 & $21.9 \pm 21.5$ \\
\hline 2 & - & 7 & $19.1 \pm 19.9$ \\
\hline 2 & + & 29 & $48.7 \pm 23.3$ \\
\hline 2 & - or + & 36 & $43.0 \pm 25.4$ \\
\hline 1 & - & 4 & $84.2 \pm 31.5$ \\
\hline 1 & t & 13 & $68.7 \pm 25.2$ \\
\hline 1 & $-o r t$ & 17 & $72.3 \pm 26.6$ \\
\hline 0 & - & 9 & $96.5 \pm 7.6$ \\
\hline 0 & + & 15 & $98.9 \pm 4.1$ \\
\hline 0 & - or + & 24 & $98.0 \pm 5.6$ \\
\hline
\end{tabular}


TABLE A-6

Mean thigh pressure indices and ankle pressure indices ( 1 SD) in normals and in the ditherent categories of patients.

The symptomatic legs of the symptomatic patients are mentioned separately.

\begin{tabular}{|c|c|c|c|c|}
\hline SYMPTOMS & & $\begin{array}{c}N \\
(\operatorname{legs})\end{array}$ & $\begin{array}{c}\text { THIOH PRESSURE INDEX } \\
\text { mear } \pm \text { SD }\end{array}$ & $\begin{array}{c}\text { ANKLE PRESSURE TNDEX } \\
\text { mean } \pm \text { SD }\end{array}$ \\
\hline NORMALS & & 56 & $1.05 \div 0.08$ & $1.08 \pm 0.08$ \\
\hline \multicolumn{5}{|l|}{ PATIENTS } \\
\hline A-symptomatic & & 26 & $1.07 \pm 0.14$ & $1.05 \pm 0.21$ \\
\hline \multirow{2}{*}{ Claudication } & all legs & 124 & $0.85 \pm 0.21$ & $0.76 \pm 0.25$ \\
\hline & sympt. legs & 77 & $0.80 \pm 0.21$ & $0.68+0.23$ \\
\hline \multirow[t]{2}{*}{ Rest pain } & all legs & 30 & $0.81 \pm 0.28$ & $0.64 \pm 0.35$ \\
\hline & sympl. legs & 15 & $0.67 \pm 0.28$ & $0.47 \pm 0.39$ \\
\hline \multirow[t]{2}{*}{ Necrosis } & all legs & 12 & $0.89 \pm 0.25$ & $0.79 \pm 0.31$ \\
\hline & sympt. legs & 6 & $0.84 \pm 0.27$ & $0.72 \pm 0.35$ \\
\hline
\end{tabular}

TABLE A-7.

Mean thigh pressure indices and ankle pressure indices (f SD) in legs of difterent angiographic classification (angioclass).

\begin{tabular}{cccc}
\hline ANGIOCLASS & $\begin{array}{c}\mathrm{N} \\
\text { (legs) }\end{array}$ & $\begin{array}{c}\text { THIOHRESSURE INDEX } \\
\text { mean SD }\end{array}$ & $\begin{array}{c}\text { ANKLE PRESSURE INDEX } \\
\text { megn }\end{array}$ \\
\hline 0 & 56 & $1.05 \pm 0.08$ & $1.08 \pm 0.08$ \\
1 & 96 & $1.02 \pm 0.13$ & $0.95 \pm 0.21$ \\
2 & 19 & $0.85 \pm 0.20$ & $0.55 \pm 0.28$ \\
3 & 38 & $0.73 \pm 0.17$ & $0.69 \pm 0.20$ \\
4 & 16 & $0.54 \pm 0.17$ & $0.37 \pm 0.18$
\end{tabular}

TABLE A.8.

Patients" performance during the treadmill walking test.

\begin{tabular}{|c|c|c|c|c|}
\hline \multirow{2}{*}{$\begin{array}{l}\text { PATIENTS } \\
\text { SYMPTOMS }\end{array}$} & \multicolumn{3}{|c|}{ REASON TO STOP THE WALKINC TEST } & \multirow[b]{2}{*}{ TOTAL. } \\
\hline & TIME CRITERIUM & CLAUDICATION & OTHLR COMPLANNS & \\
\hline A-symplomatic & $\|$ & 1 & $\mathbb{1}$ & 1.3 \\
\hline Claudication & 9 & 50 & 2 & $61^{*}$ \\
\hline Rest pain & 1 & 12 & 1 & $1: 4 *$ \\
\hline Necrosis & 3 & 3 & 0 & 6 \\
\hline TOTAL & 24 & 66 & 4 & 94 \\
\hline
\end{tabular}

* Two patients were excluded from the rest because of contra-indications. 
TABLE A.9.

Marn claudication time and maximum walking time in seconds $( \pm S D$ ) for the different categories of patients measured during the treadmill walking test.

\begin{tabular}{|c|c|c|c|c|}
\hline $\begin{array}{l}\text { PATIENTS } \\
\text { SYMPTOMS }\end{array}$ & $N$ & $\begin{array}{c}\text { CLAUDICATION TIME } \\
\text { mearh } \pm \mathrm{SD}\end{array}$ & MAX & $\begin{array}{l}\text { WALKINGITIME } \\
\text { mot } 1 \text { SD }\end{array}$ \\
\hline A-symplomatic & 1 & 180 & & 240 \\
\hline Clatodication & $54 \%$ & $84 \pm 52$ & & $177 \pm 83$ \\
\hline Rest pain & 12 & $67 \pm 40$ & & $126 \pm 73$ \\
\hline Necrosis & 3 & $87 \pm 49$ & & $150 \pm 95$ \\
\hline
\end{tabular}

* Includes 4 patients who developed clatication but stopped the walking test only berause of the criterion.

TABLE A-1O.

Mean scores of the ankle pressure response ( $\pm S D$ ) and the numbers of legs with the various scores. in normals and in the different patien categories.

The sympomatic legs of the symptomatic patients are mentioned separately.

\begin{tabular}{|c|c|c|c|c|c|c|c|c|c|c|}
\hline \multirow[t]{2}{*}{ SYMPTOMS } & & \multirow{2}{*}{$\underset{(\operatorname{leg} s)}{N}$} & \multicolumn{4}{|c|}{ ANKLE PRESSURE } & \multicolumn{3}{|c|}{ RESPONSE } & \multirow{2}{*}{$\begin{array}{c}\text { AVERACE OF THE } \\
\text { RESPONSE SCORE } \\
\text { MEA } \pm=\text { SO" }\end{array}$} \\
\hline & & & 0 & 1 & 2 & 3 & 4 & 5 & 9 & \\
\hline WORMALS & & $5 x$ & 52 & 3 & 1 & 0 & 0 & 0 & 0 & $0.1 \pm 0.3$ \\
\hline \multicolumn{11}{|l|}{ PATIENTS } \\
\hline A-sympromatic & & 26 & 16 & 7 & 1 & 1 & 1 & 0 & 0 & $0.6 \pm 1.0$ \\
\hline \multirow[t]{2}{*}{ Clatudication } & all legs & 124 & 22 & 13 & 29 & 36 & 3 & 15 & 6 & $2.2 \pm 1.5$ \\
\hline & sympt. legs & 77 & 6 & 5 & 16 & 30 & 3 & 13 & 4 & $2.8 \pm 1.4$ \\
\hline \multirow[t]{2}{*}{ Rest paing } & all legs & 30 & 4 & 1 & 7 & 6 & 1 & 9 & 2 & $2.9 \pm 1.8$ \\
\hline & sympt. legs & 15 & 2 & 0 & 1 & 2 & 1 & 8 & 1 & $3.7 \pm 1.8$ \\
\hline \multirow[t]{2}{*}{ Necrosis } & all legs & 1.2 & 4 & 0 & 1 & 4 & 0 & 3 & 0 & $2.4 \pm 2.0$ \\
\hline & sympt. legs & 6 & 2 & 0 & 0 & 2 & 0 & 2 & 0 & $2.7 \pm 2.2$ \\
\hline
\end{tabular}

Response $=9$ excluded from the calculation

TABLA A I .

Mean seores of the ank le pressure response (t SD) and the number of legs with their scores for the diferent angiographe cassifications (angioclass).

\begin{tabular}{|c|c|c|c|c|c|c|c|c|c|}
\hline \multirow[t]{2}{*}{ ANGIOCIASS } & \multirow{2}{*}{$\begin{array}{c}N \\
\text { (legs) }\end{array}$} & \multicolumn{7}{|c|}{ ANK1.E PRESSURE RESPONSE } & \multirow{2}{*}{$\begin{array}{l}\text { AVERACHE OE THE } \\
\text { RESPONSE SCORE } \\
\text { man + "SD" }\end{array}$} \\
\hline & & 0 & : & 2 & 3 & 4 & $s$ & 9 & \\
\hline 0 & 56 & 52 & 3 & 1 & 0 & 0 & 0 & 0 & $0.1+0.3$ \\
\hline 1 & 96 & 42 & 13 & 20 & 13 & 1 & 2 & 5 & $1.2 \pm 1.3$ \\
\hline 2 & 19 & 1 & i & 4 & 6 & 0 & 7 & 0 & $3.3 \pm 1.6$ \\
\hline 3 & 38 & 1 & 3 & 13 & 17 & 0 & 3 & 1 & $2.6 \pm 1.0$ \\
\hline 4 & 16 & 0 & 0 & 0 & 3 & 2 & 11 & 0 & $4.5 \pm 0.8$ \\
\hline
\end{tabular}

\footnotetext{
* Response $=9$ excluded from the calculation
} 
TABLE A 12.

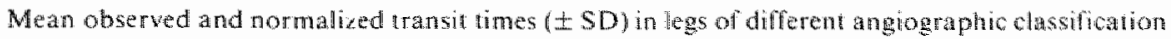
(anglioclass).

\begin{tabular}{|c|c|c|c|}
\hline ANGIOCLASS & $\mathrm{N}$ & $\begin{array}{c}\text { TRANSUT TIME } \\
\text { OBSERYED (ms) } \\
\text { mean }=\mathrm{SD}\end{array}$ & $\begin{array}{c}\text { TRANSTT TME } \\
\text { NORMALIZED } \\
\text { mean }+ \text { SD }\end{array}$ \\
\hline 0 & 47 & $27 \pm 10$ & $150 \pm$ \\
\hline 1 & 73 & $16=14$ & $126 \pm 98$ \\
\hline 2 & 16 & $15 \pm 7$ & $110+58$ \\
\hline 3 & 32 & $31 \pm 21$ & $218+180$ \\
\hline 4 & 5 & $36+39$ & $407 \pm 448$ \\
\hline
\end{tabular}

TABLE A-13.

Numbers of legs with different qualitative Doppler signal scores (Thulesius) at the fenoral and the ankle level by angographic classification of the legs (angioclass).

\begin{tabular}{|c|c|c|c|c|c|c|}
\hline \multirow[t]{2}{*}{ ANGIOCLASS } & & \multicolumn{4}{|c|}{ QUALITATIVE DOPPLER SCORE } & \multirow[b]{2}{*}{ TOTAL } \\
\hline & & 3 & 2 & 1 & 0 & \\
\hline \multirow[t]{2}{*}{0} & femoral & 56 & 0 & 0 & 0 & 56 \\
\hline & ankle & 53 & 2 & 1 & 0 & 56 \\
\hline \multirow[t]{2}{*}{1} & femoral & 92 & 3 & 1 & 0 & 96 \\
\hline & ankle & 51 & 26 & 16 & 3 & 96 \\
\hline \multirow[t]{2}{*}{2} & femoral & 14 & 3 & 1 & 1 & 19 \\
\hline & ankle & 2 & 3 & 8 & 6 & 19 \\
\hline \multirow[t]{2}{*}{3} & femoral & 14 & 13 & 9 & 2 & 38 \\
\hline & ankte & 8 & 14 & I: & 5 & 38 \\
\hline \multirow[t]{2}{*}{4} & femoral & 2 & 5 & 8 & 1 & 16 \\
\hline & anthle & 0 & 1 & 5 & 10 & 16 \\
\hline
\end{tabular}

TABLE A-14.

Mean inflow scores $( \pm S D$ ) of the patients" legs with different qualitalive femoral Doppler signal scores (Thulesius).

\begin{tabular}{ccc} 
QUALITATIEE FEORAL & N & INFLW SCORE \\
DOPPLER SCORE & Meas & SO \\
\hline 3 & 127 & $26.1 \pm 23.7$ \\
2 & 25 & $73.6 \pm 29.8$ \\
1 & 20 & $85.3 \pm 26.7$ \\
0 & 5 & $87.4 \pm 28.2$
\end{tabular}


TABLE $A-15$

Mean values if SDI of the Doppler signal parameter MAXFF.

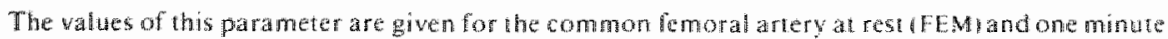
(FEM-l) and three minutes after release of thigh occlusion (FEM-3) and for the signal at the ANKLE level and further specified for the different angiogaphic classes of legs (angioctass).

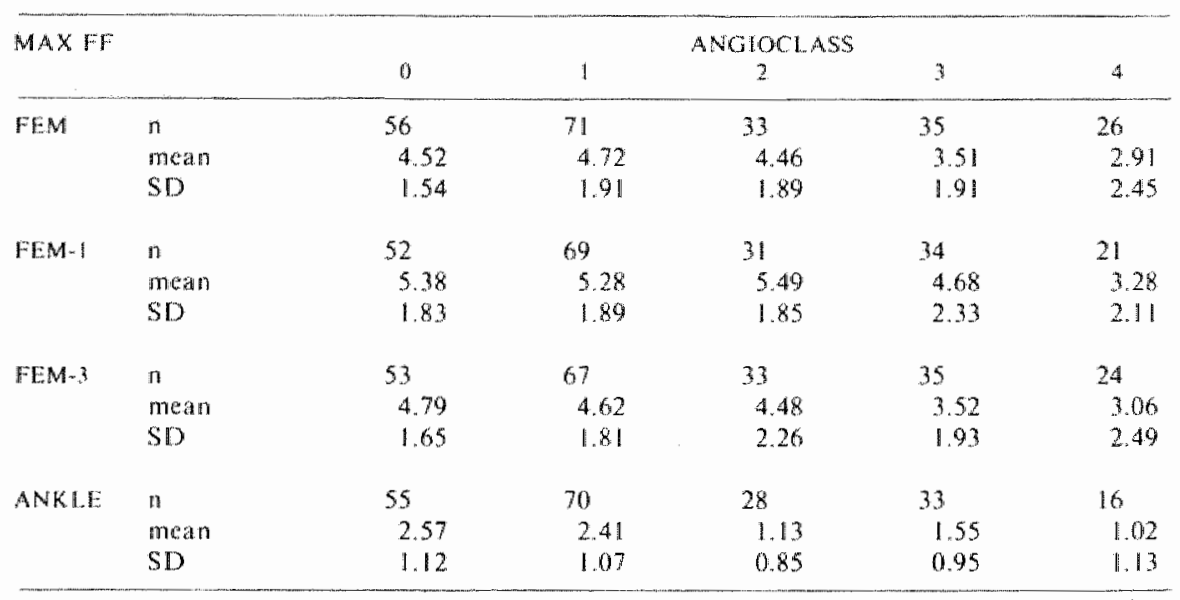

TABLE A-16.

Mean values ( \pm SD) of the Doppler signal parameter MEAN FF.

Details of table design similar wo those of Table A-15.

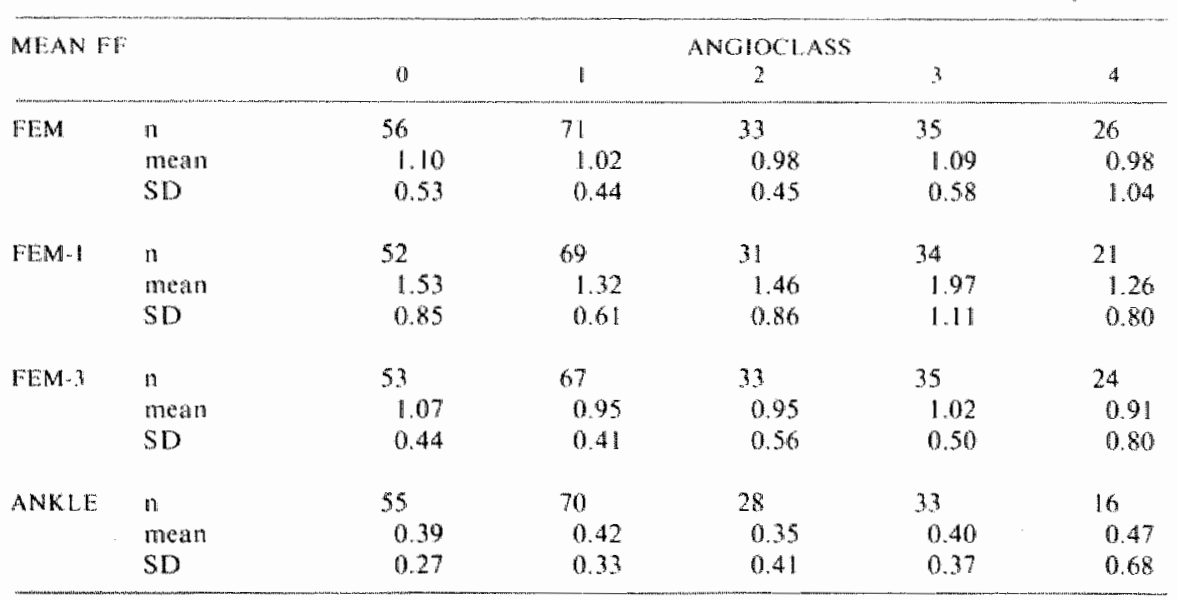


TABLE A. 17

Mean values (t SD) of the Doppler signal parameter MAX REV.

Details of table design similar to those of Table A-15.

\begin{tabular}{|c|c|c|c|c|c|c|}
\hline \multicolumn{2}{|c|}{ MAXREV } & \multicolumn{5}{|c|}{ ANGIOCLASS } \\
\hline \multirow[t]{3}{*}{ FEM } & กั้ & 56 & $7 \mathbb{1}$ & 33 & 35 & 26 \\
\hline & mean & -1.81 & -1.46 & -0.80 & -0.35 & -0.10 \\
\hline & $\mathrm{SD}$ & 0.71 & 0.72 & 0.50 & 0.63 & 0.84 \\
\hline \multirow[t]{3}{*}{ FEM-I } & $\mathrm{n}$ & 52 & 69 & 31 & 34 & 21 \\
\hline & mean & -1.39 & -1.21 & -0.62 & 0.46 & 0.21 \\
\hline & SD & 0.97 & 0.83 & 1.12 & 1.22 & 0.94 \\
\hline \multirow[t]{3}{*}{ FEM-3 } & $\mathrm{ta}$ & 53 & 67 & 33 & 35 & 24 \\
\hline & mean & -1.92 & -1.48 & -1.02 & -0.55 & -0.25 \\
\hline & $\mathrm{SD}$ & 0.65 & 0.74 & 0.48 & 0.71 & 0.71 \\
\hline \multirow[t]{3}{*}{ ANKLE } & $\mathrm{n}$ & 55 & 70 & 28 & 33 & 16 \\
\hline & mean & -0.73 & -0.46 & 0.05 & -0.09 & 0.23 \\
\hline & SD & 0.39 & 0.51 & 0.34 & 0.37 & 0.44 \\
\hline
\end{tabular}

TABLE A- $\| B$

Mean values ( $\pm \mathrm{SD}$ ) of the Doppler signal parameter $\mathrm{R} / \mathrm{F}$.

Details of table design similar to those of Table A-15.

\begin{tabular}{|c|c|c|c|c|c|c|}
\hline \multirow[t]{2}{*}{$R / F$} & & \multicolumn{5}{|c|}{ AMIOCL MSS } \\
\hline & & 0 & 1 & 2 & 3 & 4 \\
\hline \multirow[t]{3}{*}{$\mathrm{FEM}$} & n & 56 & 71 & 33 & 35 & 26 \\
\hline & mean & -0.43 & -0.35 & -0.21 & -0.07 & -0.01 \\
\hline & $\mathrm{SD}$ & 0.1 .7 & 0.22 & 0.15 & 0.16 & 0.23 \\
\hline \multirow[t]{3}{*}{ FEM-1 } & n: & 52 & 69 & 31 & 34 & 21 \\
\hline & ntiean & -0.31 & -0.25 & -0.12 & 0.11 & 0.13 \\
\hline & $S D$ & 0.21 & 0.20 & 0.20 & 0.25 & 0.24 \\
\hline \multirow[t]{3}{*}{$F \mathrm{E}-3$} & $\mathrm{n}$ & 53 & 67 & 33 & 35 & 24 \\
\hline & mean & -0.42 & -0.35 & -0.27 & -0.113 & -0.01 \\
\hline & $S D$ & 0.11 & 0.21 & 0.18 & 0.49 & 0.23 \\
\hline \multirow[t]{3}{*}{ ANKLE } & $n$ & 55 & 70 & 28 & 33 & 16 \\
\hline & mean & -0.32 & -0.20 & 0.04 & -0.08 & 0.15 \\
\hline & $\mathrm{SD}$ & 0.17 & 0.21 & 0.22 & 0.21 & 0.23 \\
\hline
\end{tabular}


TABLE A-19.

Mean values ( $\mathrm{SD}$ ) of the Doppler signal parameter $\mathrm{Pl}$

Defalls of table design similar to those of Table A-15.

\begin{tabular}{|c|c|c|c|c|c|c|}
\hline \multirow[t]{2}{*}{$P \|$} & & \multicolumn{5}{|c|}{ ANGIOCLASS } \\
\hline & & 0 & 1 & 2 & 3 & 4 \\
\hline \multirow[t]{3}{*}{ FEM } & $\mathrm{n}$ & 50 & 67 & 34 & 37 & 27 \\
\hline & mean & 12.61 & 11.79 & 8.53 & 401 & 4.69 \\
\hline & $\mathrm{SD}$ & 7.52 & 8.70 & 6.49 & 221 & 3.94 \\
\hline \multirow[t]{3}{*}{ FEM-1 } & $\mathrm{n}$ & $5 \|$ & 65 & 32 & 36 & 23 \\
\hline & mean & 9.02 & 7.97 & 5.91 & 2.93 & 2.80 \\
\hline & $\mathrm{SD}$ & 6.67 & 5.11 & 3,30 & 2.61 & 2.37 \\
\hline \multirow[t]{3}{*}{ FEM-3 } & $\mathrm{n}$ & 52 & 61 & 33 & 36 & 27 \\
\hline & mean & 14.94 & 12.12 & 9.57 & 5.31 & 4.83 \\
\hline & $S \mathrm{D}$ & 7.86 & 8.50 & 4.87 & 3.72 & 5.19 \\
\hline \multirow[t]{3}{*}{ ANKLE } & $n$ & 48 & 66 & 34 & 37 & 27 \\
\hline & mean & 16.37 & 11.30 & 4.27 & 6.00 & 2.31 \\
\hline & $S D$ & 7.78 & 7.01 & 4.14 & 4.95 & 327 \\
\hline
\end{tabular}

TABLE A-20.

Mean values ( 1 SD) of the Doppler signal parameter UST (H).

Details of table design similat to those of Table A-15.

\begin{tabular}{|c|c|c|c|c|c|c|}
\hline \multirow[t]{2}{*}{ UST (H) } & & \multicolumn{5}{|c|}{ ANGIOCLASS } \\
\hline & & 0 & 1 & 2 & 3 & 4 \\
\hline \multirow[t]{3}{*}{ FEM } & n & 56 & 71 & 33 & 35 & 26 \\
\hline & mean & 122 & 113 & 106 & 162 & 155 \\
\hline & $\mathrm{SD}$ & 21 & 22 & 36 & 43 & 61 \\
\hline \multirow[t]{3}{*}{ FEM-1 } & $\mathrm{n}$ & 52 & 69 & 31 & 34 & 21 \\
\hline & mean & 1.34 & 130 & 116 & 207 & 207 \\
\hline & $\mathrm{SD}$ & 21 & 38 & 30 & 96 & 109 \\
\hline \multirow[t]{3}{*}{ FEM-3 } & $\mathrm{n}$ & 53 & 67 & 33 & 35 & 24 \\
\hline & mean & 122 & 114 & 110 & 156 & 159 \\
\hline & $\mathrm{SD}$ & 19 & 28 & 28 & 39 & 58 \\
\hline \multirow[t]{3}{*}{ ANKLIE } & n & 55 & 70 & 28 & 33 & 16 \\
\hline & mean & 86 & 88 & 130 & 124 & 216 \\
\hline & $\mathrm{SD}$ & 17 & 29 & 72 & 44 & 145 \\
\hline
\end{tabular}


TABLE A-2I.

Mean walues $( \pm S D)$ of the doppler signal parameter UsT (DER

Detalls of table design simillar 10 those of Table A-15.

\begin{tabular}{|c|c|c|c|c|c|c|}
\hline \multirow{2}{*}{\multicolumn{2}{|c|}{ UST (DER) }} & \multicolumn{5}{|c|}{ ANGIOCLASS } \\
\hline & & 0 & 1 & 2 & 3 & 4 \\
\hline \multirow[t]{3}{*}{ FEM } & $\mathrm{n}$ & 56 & 71 & 33 & 35 & 26 \\
\hline & mean & 126 & 116 & 108 & 160 & 155 \\
\hline & $\mathrm{SD}$ & 20 & 22 & 27 & 41 & 59 \\
\hline \multirow[t]{3}{*}{ FEM-1 } & $n$ & 52 & 69 & 3 & 34 & 21 \\
\hline & mean & 139 & 125 & 118 & 201 & 197 \\
\hline & $\mathrm{SD}$ & 21 & 26 & 26 & 85 & 98 \\
\hline \multirow[t]{3}{*}{ FEM-3 } & n & 53 & 67 & 33 & 35 & 24 \\
\hline & mean & 125 & $\| \pi$ & 114 & 155 & 160 \\
\hline & $\mathrm{SD}$ & 18 & 22 & 26 & 36 & 58 \\
\hline \multirow[t]{3}{*}{$\triangle N K L E$} & $\mathrm{n}$ & 55 & 70 & 28 & 33 & 16 \\
\hline & mean & 86 & 90 & 151 & 124 & 224 \\
\hline & $\mathrm{SD}$ & 14 & 25 & 89 & 40 & 155 \\
\hline
\end{tabular}

TABLE A-22.

Mean values (I SD) of the Doppler signal parameter DST

Details of table design similar to those of Table A-15.

\begin{tabular}{|c|c|c|c|c|c|c|}
\hline \multirow[t]{2}{*}{ DST } & & \multicolumn{5}{|c|}{ ANGIOCLASS } \\
\hline & & 0 & 1 & 2 & 3 & 4 \\
\hline \multirow[t]{3}{*}{ FEM } & $n$ & 56 & $7 !$ & 33 & 35 & 26 \\
\hline & mean & 147 & 150 & 168 & 197 & $23 y$ \\
\hline & $\mathrm{SD}$ & 22 & 32 & 40 & 58 & 118 \\
\hline \multirow[t]{3}{*}{ FEN-1 } & $\mathrm{n}$ & 52 & 69 & 31 & 34 & 21 \\
\hline & mean & 157 & 158 & 166 & 274 & 302 \\
\hline & $\mathrm{SD}$ & 30 & 34 & 40 & 117 & 144 \\
\hline \multirow[t]{3}{*}{$F E M-3$} & $n$ & 53 & 67 & 33 & 35 & 24 \\
\hline & mean & 146 & 142 & 165 & 201 & 239 \\
\hline & $\mathrm{SD}$ & 19 & 31 & 39 & 60 & 90 \\
\hline \multirow[t]{3}{*}{ ANKLE } & $\mathrm{n}$ & 55 & 70 & 28 & 33 & 16 \\
\hline & mean & 94 & 114 & 205 & 166 & 280 \\
\hline & $\mathrm{SD}$ & 20 & 40 & 112 & 60 & 202 \\
\hline
\end{tabular}


TA

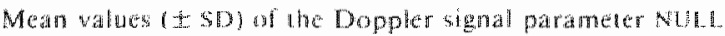

Details of table design simar to those of Table A 15

\begin{tabular}{|c|c|c|c|c|c|c|}
\hline \multirow[t]{2}{*}{ NULL. } & & \multicolumn{5}{|c|}{ ANGIOCHASS } \\
\hline & & 0 & $t$ & 2 & 3 & 4 \\
\hline \multirow[t]{3}{*}{ FEM } & $n$ & 56 & $7 \|$ & 33 & 35 & 26 \\
\hline & mean & 26 & 41 & 44 & 23 & 27 \\
\hline & $\mathrm{SD}$ & 18 & 20 & 22 & 23 & 26 \\
\hline \multirow[t]{3}{*}{$\mathrm{FEM}-1$} & ni & 52 & 69 & 31 & 34 & 21 \\
\hline & mean & 16 & 28 & 29 & 12 & 8 \\
\hline & 50 & 15 & 24 & 25 & 19 & 17 \\
\hline \multirow[t]{3}{*}{ FEM-3 } & $n$ & 53 & 67 & 33 & 35 & 24 \\
\hline & mean & 29 & 46 & 52 & 26 & 26 \\
\hline & $\mathrm{SD}$ & 16 & 18 & 16 & 23 & 24 \\
\hline \multirow[t]{3}{*}{ ANKLE } & $n$ & 55 & 70 & 28 & 33 & 16 \\
\hline & mean & 58 & 60 & 47 & 49 & 41 \\
\hline & $\mathrm{SD}$ & 23 & 23 & 27 & 27 & 32 \\
\hline
\end{tabular}

TABLEA 24

Numeratte parameters of the Doppler signal of the common femoral artery at rest (FEMt which have a signilicant difference $(p<0.001)$ between their mean walues for the legs in crosswise indicated angiographic classes dangioclass).

\begin{tabular}{|c|c|c|c|c|}
\hline ANGIOCLASS & 1 & 2 & 3 & 4 \\
\hline 0 & NLLL & $\begin{array}{l}M A X R E V \\
R / F \\
\text { NUIA. }\end{array}$ & $\begin{array}{l}\text { MAX REK } \\
\text { PI } \\
R / F \\
\text { UST (II) } \\
U S T(D R R) \\
\text { DST }\end{array}$ & $\begin{array}{l}\text { MAXFE } \\
\text { MAXREV } \\
\text { PI } \\
\text { RE } \\
\text { UST [H] } \\
\text { DST }\end{array}$ \\
\hline $\mathbb{H}$ & & $M A X R E V$ & $\begin{array}{l}\text { NAX REK } \\
\text { PI } \\
\text { R/F } \\
\text { UST (H) } \\
\text { UST (DER } \\
\text { DST } \\
\text { NULL }\end{array}$ & $\begin{array}{l}\text { MAXFE } \\
\text { MAXREY } \\
\text { PI } \\
\text { R/S } \\
\text { UST (H) } \\
\text { UST (DER) } \\
\text { DST }\end{array}$ \\
\hline 2 & & & $\begin{array}{l}\mathrm{Pl} \\
\mathrm{R} / \mathrm{F} \\
\text { UST }(\mathrm{H}) \\
\text { UST (DER) } \\
\text { NUII! }\end{array}$ & $\begin{array}{l}\text { MAX REV } \\
\text { R/F } \\
\text { UST (H) } \\
\text { UST (DER })\end{array}$ \\
\hline
\end{tabular}


TABLE A-25.

Numerate parameters of the Doppler signal of the common fomoral artery one mimute after retense

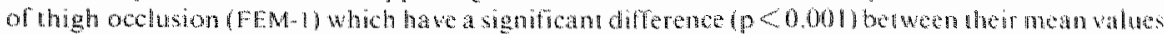
for the legs in crosswise indicated angiographic classes (angioclassi.

\begin{tabular}{|c|c|c|c|c|}
\hline ANCHOCLASS & 1 & 2 & 3 & 4 \\
\hline 0 & - & $\begin{array}{l}\mathrm{R} / \mathrm{F} \\
\mathrm{UST}(\mathrm{DHR})\end{array}$ & $\begin{array}{l}\text { MAX REV } \\
\text { PI } \\
\text { RF } \\
\text { UST (H) } \\
\text { UST (DFR) } \\
\text { DST }\end{array}$ & $\begin{array}{l}\text { MAX WE } \\
\text { MAX REV } \\
\text { PI } \\
\text { RPt } \\
\text { UST (HI) } \\
\text { UST (DER) } \\
\text { OST }\end{array}$ \\
\hline 1 & & - & $\begin{array}{l}\text { MAX RUY } \\
\text { PI } \\
\text { R/F } \\
\text { UST (H) } \\
\text { UST (DER) } \\
\text { DST }\end{array}$ & $\begin{array}{l}\text { MAXFF } \\
\text { MAXREV } \\
\text { PI } \\
\text { R/H } \\
\text { USE (HU) } \\
\text { UST (DER) } \\
\text { DST }\end{array}$ \\
\hline 2 & & & $\begin{array}{l}\text { MAX Rl: } \\
\text { PI } \\
\text { RAF } \\
\text { DST (H) } \\
\text { DST (DFR) } \\
\text { DST }\end{array}$ & $\begin{array}{l}\text { MAXFI } \\
\text { PI } \\
\text { RAF } \\
\text { UST (HI } \\
\text { UST (DRR) } \\
\text { DST }\end{array}$ \\
\hline
\end{tabular}

3 
TABLE A-26.

Numerate parameters of the Doppler signal of the cormmon femoral artery thee minutes after releatse of thigh ocelwion (FEM-3) which have a significant difference $(p<0.001)$ berween their mean values for the legs in crosswise indicated angiographic classes (angioclass).

\begin{tabular}{|c|c|c|c|c|}
\hline ANGIOCLASS & 1 & 2 & 3 & 4 \\
\hline 6). & $\begin{array}{l}\text { MAX REW } \\
\text { R/F } \\
\text { NULL }\end{array}$ & $\begin{array}{l}\text { MAX REV } \\
\text { PI } \\
\text { R/F } \\
\text { NULL. }\end{array}$ & $\begin{array}{l}\text { MAX REV } \\
\mathrm{Pl} \\
\mathrm{R} / \mathrm{F} \\
\text { UST (H) } \\
\text { UST (OER) } \\
\text { DST }\end{array}$ & $\begin{array}{l}\text { MAXFF } \\
\text { MAX REV } \\
\text { PI } \\
\text { R/F } \\
\text { UST (H) } \\
\text { UST (DER) } \\
\text { DST }\end{array}$ \\
\hline $\mathbb{1}$ & & - & $\begin{array}{l}\text { MAX REV } \\
\text { FI } \\
\text { R/F } \\
\text { UST (HI) } \\
\text { UST (DER) } \\
\text { DST } \\
\text { NULL }\end{array}$ & $\begin{array}{l}\text { MAX REV } \\
\text { PI } \\
\text { R/F } \\
\text { UST (H) } \\
\text { UST (DER) } \\
\text { DST } \\
\text { NULL. }\end{array}$ \\
\hline 2 & & & $\begin{array}{l}\text { MAX FE } \\
\text { PI } \\
\text { UST (HT) } \\
\text { UST (OER) } \\
\text { NULL }\end{array}$ & $\begin{array}{l}\text { MAX FF } \\
\text { R/F } \\
\text { UST (H) } \\
\text { USY (DER) } \\
\text { DST } \\
\text { NULL. }\end{array}$ \\
\hline 3 & & & & - \\
\hline
\end{tabular}

TABLE A-27.

Numerate parameters of the Doppler signal at the ankle level which have a significant difference $(p<0.001)$ between their mean values for the legs in crosswist indicated angiographic classes (angioclass).

\begin{tabular}{|c|c|c|c|c|}
\hline ANGIOCLASS & 1 & 2 & 3 & 4 \\
\hline 0 & $\begin{array}{l}\mathrm{PA} \\
\mathrm{R} / \mathrm{F} \\
\mathrm{DS} \mathrm{l}^{\circ}(\mathrm{DER})\end{array}$ & $\begin{array}{l}\text { MAX FE } \\
\text { MAX REV } \\
\text { PI } \\
\text { R/F } \\
\text { DST }\end{array}$ & $\begin{array}{l}\text { MAX FF } \\
\text { MAX REV } \\
\text { PI } \\
\text { R/F } \\
\text { UST OH) } \\
\text { UST (DER) } \\
\text { DST }\end{array}$ & $\begin{array}{l}\text { MAX FF } \\
\text { MAX REV } \\
\text { PI } \\
\text { R/F } \\
\text { UST (H) } \\
\text { UST (DER) } \\
\text { DST }\end{array}$ \\
\hline 1 & & $\begin{array}{l}\text { MAXFF } \\
\text { MAX REY } \\
\mathrm{PI} \\
\mathrm{M} / \mathrm{F} \\
\text { UST ODER } \\
\mathrm{DST}\end{array}$ & $\begin{array}{l}\text { MAX FF } \\
\text { MAX REV } \\
\text { PI } \\
\text { UST (H) } \\
\text { UST (DER) } \\
\text { DST }\end{array}$ & $\begin{array}{l}\text { MAX FF } \\
\text { MAX REV } \\
\text { PI } \\
\text { R/F } \\
\text { UST (H) } \\
\text { UST (DER) }\end{array}$ \\
\hline 2 & & & - & - \\
\hline 3 & & & & - \\
\hline
\end{tabular}




\section{REFERENCES}

ALTSTAEDT F, STORZ LW, RÜCKERT U. Erste Erfahrungen mit einer semiquantitativen Analyse won Doppler-Fluss-Kurwen in der Gefasschirurgie. In: UltraschallDoppler-Diagnostik in der Angiologie. (Eds. KRIESSMANN A. BOLLINGER A). Thieme, Stuttgart 1979; pp. 67-79.

ANDEL GJ van. Transluminal iliac angioplasty: long term results. Radiology.1980; 135: 607-611.

ANDEL GJ van, KREPEL VM. De behandeling van stenosen in de arteriae iliacae met dilatatiecatheters (Dotter-methode). Ned. Tijdschr Geneeskd. 1979; 123:873878 .

ANGELIDES NS, NICOLAIDES AN, EASTCOTT HHG. The value of Dopplet velocity tracings in femoropopliteal disease. In: Ultraschall-Doppler-Diagnostik in der Angiologie. (Eds. KRIESSMANN A, BOLLINGER A). Thieme, Stuttgart 1979; pp. $17-20$.

ATTINGER EO, ANNÉ A, McDONALD DA. Use of Fourier series for the analysis of biological systems. Biophys J 1966; $6: 291 \times 304$.

BAIRD RN, LUSBY RJ, BIRD DR, GIDDINGS AEB, SKIDMORE R, WOODCOCKJP, HORTON RE, PEACOCK JH. Pulsed Doppler angiography in lower limb arterial ischemia. Surgery $1979 ; 86 ; 818-825$.

BAIRD RN, BIRD DR, CLIFFORD PC, LUSBY RJ, SKIDMORE R, WOODCOCK JP. Upstream stenosis. Its diagnosis by Doppler signats from the femoral artery. Arch Surg $1980 ; 115: 1316-1322$.

BAKER JD. Poststress Doppler ankle pressures. A comparison of treadmill exercise with two other methods of induced hyperemia. Arch Surg 1978;113:1171-1173.

BARDACH G. Eine neue Methode zur Direktmessung des aortoremoralen Druckgradienten im Rahmen der translumbalen Aortographie. Fortschr Röntgenstr 1978; $128: 317-318$.

BARENDSEN GJ. Blood flow in human extremities at rest, after arterial occlusion and after exercise. Thesis, Groningen 1973.

BARNHORST DA, BARNER HB. Prevalence of congenitally absent pedal pulses. N Engl J Med 1968; $278: 264-265$.

BERNINK PILM. Parameters van de circulatie in de onderste extremiteiten met betrek-

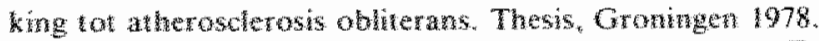

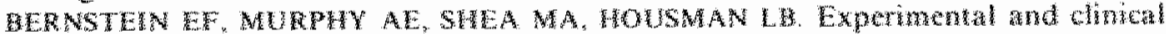

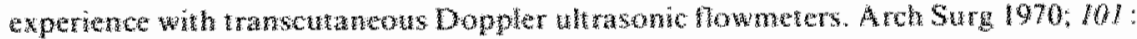
$21-25$.

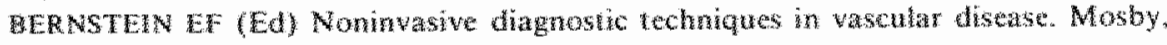

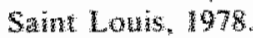

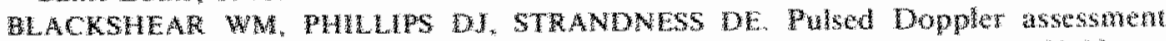
of nomal human femoral antery veociny patuens. I Surg Res $1979.27: 73-8$ a.

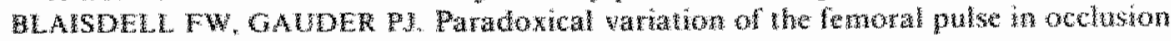
of the ilac artery. Surgery $196: 50: 529-532$.

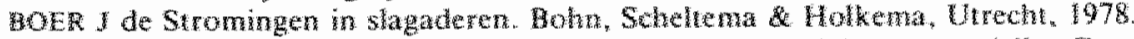

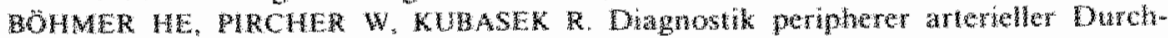

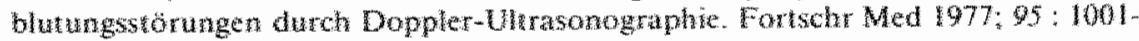
1006 .

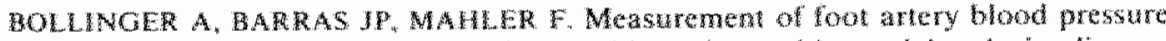

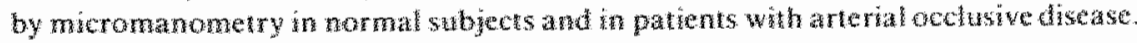
Crenkation 1976 : $5: 506-512$. 
DOLLIFGER A.MAHLER F. ZEHENDER O. KOMbinerte Druck-und DUTMUSsmesurgen in der Beurtelung arterielles Durchblutungsionungen. Disch Med Wochenschr 1970;95: 1039-1043.

BOWE GE. HAYES AC. SLAYMAKER EE. PARNES RW. Value of segmental limb blood pressures in predicting result of aortofemoral bypass. Am J Surg $1976 ; 132$ : $733-738$

BONE GE. AMMONS D. Characteriation of experimental arterial stenosis by numerate analysis of the Doppler velocity waveform. Surg Forum 1978, 24: $208-209$.

BRENER BJ, RAINES JK, DARLING RC. AUSTEN WG. Measurement of syslolic femora! arterial pressure during reactive hyperemia. An estimate of aortoiliac discase. Circulation 1974; supp/ II: $259-267$.

BROWN IM, WHORSKI ZT, WOODCOCK JP, MORRIS SJ. Transferwuction modelling of anteries. Med Biol Eng Compur 1978; 16:161-164.

BRUIJNINCKX CMA. Prem en postoperatieve evaluatie van Claudicatio intermitens met behulp van een Doppler-flowmeter. Thesis, Nijmegen 1976.

BRUINS SLOT H. Doppler studies in the femorompoliteal pathway. Thesis, Maastricht 1981 .

BUCHEM FSP wan. De pathogenese van atherosclerotische complicaties bij de mens. Ned Tijolschr Geneeskd 1967; 11/: 1793-1801.

BUTH $\mathbf{j}$. Doppler-bloeddrukmeting aan de enkel bij perifeer arterbel vaatliden: een nieume aanwinst in de watchirugie. Ned Tijdschr Geneeskd 1975; 19: 388-397.

BuTH J. Het vasculair laboratorium. Thesis, Amsterdam 1978.

CARTER SA. Indireet systolic presslares and pulse waves in anterial occlusive disease of the lower extremties. Circulation 1968; $37: 624-637$.

CARTLR $S$ A. Clinical measurement of systolic pressures in limbs witharterial occlusive disease. JAMA 1969:207: 1869-1874

CARTER SA. Response of ankle systolic pressure to legexercise in mild or questionable arterial disease. $N$ Engl $\mathbb{I}$ Med $1972 ; 287: 578-582$.

CASTANEDA-ZUNIGA W, KNIGHT L, FORMANEK A. MOORE R, D'SOUZA V. AMPLATZ K. Hemodynamic assessment of obstructive Aortoiliac disease. Am J Roentgenol 1976; $127: 559-561$

CAVE FD. WALKER A, NAYLOR GP, CHARLESWORTH D. The hydraulic impedance of the lower limb: its relevance to the success of bypass operations for occlustion of the superficial femoral artery. Br J Surg 1976;63:408-4l2.

CHAMBERLAIN J, HOUSLEY E, MACPHERSON AIS. The relationship between ultrasound assessment and angiography in occlusive arterial disease of the lower linb. Br J Surg 1975; $62: 64 \sim 67$.

CHARLESWORTH D. HARRIS PL, GAVE FD. TAYLOR L. Undetected artomina insulficiency: a reason for early lailure of saphenous vein bypass grafts for obstruction of the stuperficial Cemoral artery. Br J Surg 1975;62:567-570.

COGHLAN BA. TAYI.OR MG. On methods hon preprocessing direction Dopplar signals to allow display of directional blood-velocity waveforms by spoctrum analysers. Med Biol Eng Comput 1978:16:549-553.

COOLEY WW, LOHNES PR. Multivaratedata analysis. Wiley. New York, 1971.

CRUMMY AB, RANKIN RS, TURNIPSEED WD, BERKOFF HA Biplane arieriography in ischemia of the lower extremity. Radiology 1978;126:111-115.

CUTAIAR CL, MARSTON A. NEWCOMBE JF. Value of cuffocclusion pressures in assessment of peripheral vasculat disease. Br Med J 1973:2:392-395.

DARLING RC, RAINES IK. BRENER BJ, AUSTEN WG. Quantitative segmental pulse volume recorder: A clinical tool. Surgery 1972;72:873-887.

DARLING RC. BREWSTER DC. HALLETI JW, DARLING RC. AOTO-iliac Heconstruc fion. Surg Clin North Am 1979; $59: 565-579$

DEAN RH. YAO JST. Hemodynamic measurements in peripheral vascular disease. Cur Probl Surg 1976; 13. nor 8 . 
DEBAKEY ME, CRAWFORD ES, COOLEY DA, MORRIS GC. Surgical considerations of occlusive disease of the abdominal Aortand lliac and Femoral arteries. Analysis of 903 cases. Ann Surg 1958: 148: 306-324.

DELIUS W. Arterieller Druck. In: Messmethoden bei arteriellen Durchblutungsstörunger. (Eds. BOLLINGER A. BRUNNER U.) Huber. Bern 1971 ; pp. $38-41$

DILLEY RB, FRONEK A. Quantitative velocity measurements in arterial disease of the lower extremity. In: Noninwasive diagnostic techniques in vascular distase. (Ed. BERNSTEIN EF.) Mosby, Saint Louis 1978; pp, 294-303.

DUDA RO, HART PE. Pattern classification and scene analysis. Willey, New York 1973 ,

EHRINGER H. Die reaktive Hyperämie nach arterieller Sperre. In: Messmethoden bel arteriellen Durchblutungssörungen. (Eds. BOLLINGER A. BRUNNER U.) Huber. Bern 1971; pp. 20-33.

EVANS DH, QUIN RO, BELI. PRF. The significance of blood pressure measurements in patients with peripheral vascular disease. Br J Surg $1980 ; 67: 238-241$.

FARBAIRN JF II Clinical manilestations of peripheral vascular disease. In: AllenBarker-Hines" Peripheral vascular disease, (Eds. JUERGENS JL. SPITTELL IA. FAIRBAIRN II JF.) Saunders, Philadelpha 1980: (5th ed) Chapler 1, p. 3-49.

FARIS IB. JAMIESON CW. The diagnosis of aorto-iliac stenosis: A comparison of thigh pressure measurement and femoral artery flow velocity profile. J Cardiowas Surg $1975 ; 16: 597-602$.

FARRAR DJ. MALINDZAK GS, JOHNSON G. Large vessel impedanee in peripheral atherosclerosis. Circulation 1977; 56 (supp 2): 171-178.

FELIX WR, SIGEL B. POPKY GL. Doppler ultrasound in the diagnosis of peripheral vascular disease. Semin Roentgenol 1975; 10:315-32l.

FITZGERALD DE, GOSLING RG. WOODCOCK JP. Grading dynamic capability of arterial collateral circulation. Lancet 1971: $I: 66-67$.

FITZGERALD DE, CARR J. Doppler ultrasound diagnosis and classification as an alternative 10 arterioguaphy. Angiology $1975 ; 26: 283-288$.

FITZGERALD DE,CARR J. Peripheral arterial disease: Assessment by arteriography and alternative noninvasive measurements. Am J Roentgenol 1977; $128: 385-388$.

FLANIGAN DP, TULLIS JP, STREETER VL, WHITEHOUSE WM, FREY WJ, STANLEY JC. Multiple subcritical arterial stenoses: effect on poststenotic pressure and flow. Ann Surg 1977; 186:663-668.

FLAX SW: WEBSTER JG, UPDIKE SJ. Notse and functional limitions of the Doppler blood flowmeter. In: Cardiovascular applications of ultrasound. (Ed. RENEMAN RS.) North-Holland Publishing, Amsterdam 1974; Chapter 2, pp. 18-31.

FRANSEN G, VERBIEST E, VERMEULEN F. Stenosegenisen in de perifere arterien. Tijoschr Geneesk (Belg) 1969;25:61-72.

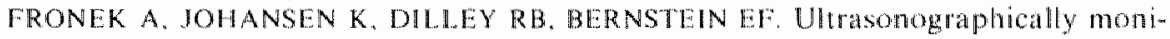
tored postocclusive reactive hyperemia in the diagnosis of peripheral anterial ocelusive disease. Circulation 1973a; $48: 149-152$.

FRONEK A, IOHANSEN KH, DHLLEY RB, BERNSTEINEF. Noninwasive physiologic lests in the diagnosis and characterization of peripheral anterial occlusive disease. Am J Surg $19736 ; 126: 205-214$.

FRONEK A. COEL M. BERNSTEIN EF. Quant tative ultrasonographic studies of lower extremity now velocities in health and disease. Circulation 1976:53:957-960.

FRONEK A COEL M. BER NSTEIN EF. The pulse-reappearalice time: an inder of over all blood flow impairment in the ischemic extremity. Surgery 1977; 81:376-381.

FRONEK A, COEL M. BERNSTEIN FF. The importance of combined multisegmental pressure and Doppler now velocity studies in the diagnosis of periphwat arterial occlusiwe disease. Surgery 1978;84:840-847

FULTONRE, STANSON AW. FORBES GS. MILLER WE. HATTERYRR WILLIAMSONB. Vascular imaging. In: Allen-Barker-Hines Peripheral vascular diseases. (Eds. JUERGENS ML. SPITTELL JA. FAIRBAIRN II JF, Saunders, Philadelphia 1980; (Sth ed) Chapter 5. pp. 139-215. 
FUSTER V, KOTTKE BA, JUERGENS JL. Atherosclerosis. In: Allen-Barker-Hines" Peripheral wascular diseases. (Eds. JURGENS JL. SPITTELL JA, FAIRBAIRN II JF.) Saunders, Philadelphia 1980; (5th ed) Chapter 6, pp. 219-235.

GARRETT WV, SLAYMAKER EE, HEINTZ SE. BARNES RW. Intraoperative prediction of symptomatic result of aorto-femoral bypass from changes in ankle pressure index. Surgery $1977 ; 82: 504-509$

GAY P. ROBERTS VC. Characterisation of post exercise pressure response curves in the pedal arteries of patients with peripheral vascular disease. J Biomed Eng 1979; $I$ : $12-16$.

GEDDES LA, TIVEY R. The importance of cuff width in measurement of blood pressure indirectly. Cardiowasc Res Cent Bull 1976; 14:69-79.

GELSEMA ES. Ispahan Users Manual (4th edition). Dept of Medical Informatics, Free University, Amsterdam. 1981.

GOSLING RG, DUNBAR G, KING DH, NEWMAN DL, SIDE CD, WOODCOCK JP. FITZGERALD DE, KEATES JS, MACMILLAN D. The quantitative analysis of occlusiwe peripheral arterial disease by a nonintrusive ultrasonic technique. Angiology $1971 ; 22$ : $52-55$

GOSLING R, KING D. WOODCOCK J. Blood-velocity waveforms in the evaluation of atheromateus changes. In: Blood now measurement (Ed. ROBERTS C.) Sector, London 1972; pp. 33-36.

GOSLING RG, KING DH. Arterial assessment by Doppler-shift ultrasound. Proc Roy Soc Med. 1974a; $67: 447-449$

GOSLING RG, KING DH. Continuous wave ultrasound as an alternative and complement to X-ray in vascular examinations. In: Cardiovascular applications of ultrasound. (Ed. RENEMAN RS) North-Holland Publishing, Amsterdam 1974b; pp. 266282.

GOSLING RG. Extraction of physiological information from spectrum-analysed Doppler-shifted continuous-wave ultrasound signals obtained non-invasively from the arterial system. In: I.E.E. Medical Electronics Monographs (Eds. HILL and WATSON) Peter Peregrinus, Stevenage 1976; monograph 21.

GOSLING RG, KING DH. Processing arterial Doppler signals for clinical data. In: Handbook of Clinical Ultrasound. (Ed. VLIEGER M. de et al) Willey, New York 1978; pp. $613-646$

GRÜNTZIG A, BRUNNER U, MEIER W, BOLLINGER A. Prä- und postoperative Messung der systolischen Knöchelarteriendrucke mit Doppler Ultraschall. In: Messmethoden bei arteriellen Durchblutungsstörungen. (Eds. BOLLINGER A, BRUNNER U.) Huber, Bern 1971; 177-187.

GRÜNTZTG A. Die Ultraschall-Doppler-Methode in der angiologischen Diagnostik Med Klin 1975; $70: 2019-2026$.

HAIMOVICI H. ESCHER DJW. Aortoiliac stenosis. Diagnostic significance of vascular hemodynamics. Arch Surg 1955; 72: 107-117.

HAIMOVIC H. Atherogenisis. Recent biological concepts and clinical implications. Am J Surg 1977; 134: 174-178.

HARPER DR, KELMAN GR, MAVOR GE, WALKER MG, WATSON AWS. Time interval between e.c.g. $R$ wave and peak flow velocity in leg arteries of normal humans. $J$ Physiol 1974; $239: 21-22 \mathrm{P}$

HARRIS PL, TAYLOR LA, CAVE FD. CHARLESWORTH D. The relationship between Doppler ultrasound assessment and angiography in occlusive arterial disease of the lower limbs. Surg Gynecol Obstet 1974;138:911-914.

HEINTZ SE. BONE GE, SLAYMAKER EE, HAYES AC, BARNES RW. Value of arterial pressure measurements in the proximal and distal part of the thigh in arterial occlusive disease. Surg Gynecol Obstet 1978; 146:337-343.

HILLESTAD LK. The peripheral blood flow in intermittent claudication. V. Plethysmographic studies. The significance of the calf blood flow at rest and in response to timed arrest of the circulation. Acta Med Scand 1963a; 174:23-41. 
HILLESTAD LK. The peripheral blood flow in intermittent clatudication. VI. Plethysmographic studies. The blood flow response to exercise with arrested and with free circulation. Acta Med Scand 1963b; 174:671-685.

HILLESTAD LK. The peripheral blood flow in intermittent claudication. VIr. The difference between the hyperemias following free and ischemic exercise and the effect of the included period of ischemia upon the latter. A comparison of the tests for evaluation of the blood flow and of various methods for gauging the hyperemia. A note on the use of plethysmography in clinical studies. Acta Med Scand 1963c;174: $687-700$.

HILLESTAD LK. The measurement of arterial blood flow by venous occlusion plethysmography. In: Vascular surgery (Ed. RUTHERFORD RB.) Saunders, Philadelphia. 1977; Chapter 8, pp. 79-85.

HOBBS IT, YAO ST, LEWIS JD. NEEDHAM TN. A limitation of the Doppler ultrasound method of measuring ankle systolic pressure. Vasa 1974:3:160-162.

HOEKS APG, RENEMAN RS, PERONNEAU PA. A multigate pulsed Doppler system with serial data-processing. IEEE Trans Son Ultrason 1981; $S U-28: 242-247$.

HOEKS APG. On the development of a multimgat pulsed Doppler system with serial data-processing. Thesis, Maastricht, 1982.

HUMMEL BW, HUMMEL BA, MOWBRY A, MAIXNER W. BARNES RW Reactive hyperemia vs treadmill exercise testing in arterial diseace. Arch Surg 1978; 113:95-98.

HUMPHRIES KN, HAMES TK, SMITH SWJ, CANNON VA, CHANT ADB. Quantiative assessment of the common femoral to popliteal arterial segment using continuous wave Doppler ultrasound. Untrasound Med Biol 1980; 6 : 99-105.

HURLOW RA, CHANDLER ST, HARDMAN J, STRACHAN CJL. The noninvasive assessment of aortoiliac disease: A comparison of dynamic isotope angiology with thigh/ brachial pressure index. Surgery 1978; 84: 278-282.

HYLKEMA BS. Tussen polspalpatie en aortografie. Thesis, Groningen 1975.

HYLKEMA BS. Diagnostiek van arteriële circulatiestoornissen in de benen door bloeddruknetingen met behulp van ultrageluid. Ned Tijdschr Geneeskd 1976; 120:733-742.

IMPARATO AM, SANOUDOS G, EPSTEIN HY, ABRAMS RM. Results in 96 aortoiliac reconstructive procedures: Preoperative angiographic and functional classifications used as prognostic guides. Surgery 1970;68:610-616.

INOKUCHI K, KUSABA A, KIYOSE T. Flow wave form analysis in vascular surgery. Am I Surg 1979; 138: 219-223.

JAUSSERAN JM, REGGI M, CASTELL ANI JC, COURBIER R. Tactique opératoire dans les artériopathies itio-fémorales. (Intérêt des épreuves d'efforts). In: Les explorations fonctionelles vasculaires (Ed, COURBIER R.) Oberval, Marseille 1976; 133-141.

JOHNSON WC. Doppler ankle pressure and reactive hyperemia in the diagnosis of arterial insufficiency.J Surg Res 1975: 18:177-180

JOHNSTON KW. TARASCHUK 1 . Validation of the role of pulisatility index in quantitation of the severity of peripheral arterial occlusive disease. Am J Surg 1976: 131: 295-297.

JOHNSTON KW. MARUZZO B, TARASCHUK I. Fourier and peak-to-peak pulsatility indices in arterial occlusive disease. In: Non-Invasive Clinical Measurement (Eds. TAYLOR D, WHAMOND J.) Pitman Medical, Tumbridge Weils, 1977a; pp. $98-104$.

JOHNSTON KW. MARUZZO BC, COBBOLD RSC. Errors and artifacts of Doppler flowmeters and their solution. Arch Surg 1977b: 12:1335-1342.

JUERGENS JL, BERNATZ PE. 9Alherosclerosis of the extremuies. In: Allen-Barker Hines' Peripheral wascular diseases. (Eds. JUERGENS JL. SPITTELL IA, FAIRBAIRN II JF.) Saunders, Philadelphia, 1980; (5th ed), Chapter 8, pp. 253-293.

KANNEL WB, SKINNER JJ, SCHWARTZ MJ, SHURTLEFF D. Intermittent claudication. Incidence in the Framingham study. Circulation $1970 ; 41: 875-883$.

KEELECA, NEIL. E. (revised) Samson Wright's Applied Physiology. Oxford University Press, London, 1963; (10th ed).

KEITZER WF. FRY WJ, KRAFT RO, DEWEESE MS. Hemodynamic mechanism for pulse changes seen in occlusive vascular disease. Surgery 1965; $57: 163-174$. 


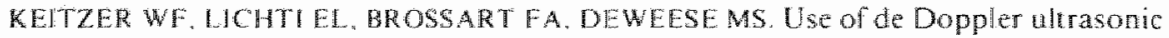
flowmete dung arterial vascular surgery. Arch Surg 1972: $105: 308-312$

KINDT GW. YOUMANS J The efrect of stricturelengh on crifical arteriat sterosis. Surg Gynecol Obstet 1969;128:729-734

KING D. COGHLAN W. GOSLING R. PICKUP A. NEWMAN D. WOODCOCK J. TranScutaneous measurement of pulse wave velocity and mean blood pressure in man. In: Blood flow measurement (Ed ROBERTS C.) Sector. London 1972: pp. 40 m3

KING LT. STRANDNESS DE. BELL JW. The hemodynamio response of the lower axtremities 10 exercise. J Surg Res 1965;5:167-171.

KIRKENDALL, WM. BURTON AC. EPSTEN FH, FREIS ED. Recomnendations for human blood pressure determination by sphygmomanometers. Circulation 1967; 36 : $980-988$.

KITSIAAR PJEHM, VEENENDAAL C van Waarde van het wenon-13-0nderzoek bij claudicatio intermittens. Ned Trijdschr Geneeskd 1977: 12: $: 208$.

KITSLAAR PIEHM. Bloeddrukmeting aan de enkel bij obstruerend arterieel vallijden. Ned Tijdschr Geneeskd 1981;125:1781-1785.

KOHLER R. Die Bedautung verschedener Projektionen fü die Beurteilung von arteriosklerotischen Veranderungen der Bauchaorta und der lliakalanterien. Fonsch Rontgenstr 1973: 118:658-662.

KROESE A.I. The contribution of muscle and skin circulation to reactive hyperaemia in the human lower limb. A study with strain gauge plethysmography. Vasa 1977:6: 9- 14.

LARSEN OA. Xenon-133 methods for determining peripheral blood llow and blood pressure in patients with occlusive arterial disease. Angiology 1972; 23: 153-162.

LASSEN NA. LINDBJERG J. MUNCK O. Measurement of blood-flow through sketal muscle by intramuscular injection of Xenon-133. Lancet 1964: $l: 686-689$

LASSEN NA, HOLSTEIN P. Use of radioisotopes in assessment of distal blood flow and distal blood pressure in anterial insurficiency. Surg Clin North Am 1974; 54: 39-55.

LEVESON SH, GUILLOU PJ, TERRY HJ GLANVILLE JN. KESTER RC. PUISE pressure wave analysis in the diagnosis of aorto-iliac disease. Ann Surg 1978; 187:161-165.

LEWIS JD, PAPATHANAIOU C, YAOST, EASTCOTT HHG. SimultaneOHS flow and pressure measurements in intermittent claudication. $\mathrm{Br}$ J Surg 1972n; 59:418-422.

LEWIS J, YAOST, EASTCOTTH. Blood low and pressure in periphenal vascular disease. In: Blood flow measurement (Ed, ROBERTS (") Sector, London 1972b; 69-73.

LEWIS JD, YAOST. Waveform and pressure measurement with a directional Doppler in the diagnosis and follow-up of peripheral arterial disease. In: Cardiowasuar applications of ultmalsund. (Ed. RENEMAN RS.) Nonh-Holland Publishing, Amsterdam 1974: pp. 294-304.

1.UDBROOK . Collateral antery tesistance in human lower limb. J Sutg Res 1966:6: $423-434$.

MACKERETH M. LENNIHANR. Ultrasonind as an aid in the dragnosis and mamagement ol intermittent clatication. Angiology 1970;2l:704-713.

MADDISON FE. Instruments and techniques for arteriography. In: Vasculat surgery (Ed. RUTHERFORD RB.) Saunders, Philadelphia 1977; Chapter 28, pp. 263-270.

MAHLER F. KOEN L, JOHANSEN KH, BERNSTEN EF, FRONEK A. POStOCOLSIOn and postexercise flow velocity and ankle pressures in normals and marathon rumners. Angiology 1976;27:721-729.

MANSJOER M, SCHMIDTKE I, ZEITLER E. Angiographische Sicherheit bed der Beurteilung von Beckenarterienstenosen. In: Aspekte der Fxtremitatenangiographie. Fehldiagnosen und Fuhlinterpretationen. Ed, ZEITLER E.) Huber, Bern 1976; pp. $157-162$

MATSUO H. NIMURA Y, KITABATAKE A. HAYASHIT. Andlysis of flow patterns in blood vessels with the directional ultasonic Doppler technique through a transculancous approach. Ipn Circ J 1973:37:735-746.

MAURIZI JL. Place de la débineróne ulrasonore dans le protocole des explorations artericlles. Thesis, Lyon 1974. 
MAY AG. DEWEESE JA. ROB CG. Hemodynamic elfects of anterial stenosis Surgery $1963 ; 53: 513-524$.

MCDONALD DA. Blood flow in arteries. Arnold. London. (Second edition) 1974.

MCLEOD FD. Multichannel pulse Doppler techniques. In: Cardiovascular applications of ultrasound. (Ed. RENEMAN RS.) North-Holland Publishing. Amsterdam 1974, Chapter 7, pp. 85-107.

MCNEIL. BJ. KEELER E, ADELSTEIN SI. Primer on certain elements of medical decision making. N Engl J Med 1975; 293:211-215.

MICHE DD, CAIN CP. Eflect of hematrocrit upon the shift in Doppler frequency. Proc Soc Biol Med 1971: 138: 768-772.

MOORE WS, HALL. AD. Unrecognized aortoiliac stenosis. A physiologicapproach to the diagnosis. Arch Surg 1971: $103: 633-638$.

MOZERSKY DI. SUMNER DS, STRANDNESS DE. LOng-term results of reconstructixe Aortoiliac surgery. An J Surg 1972: 123:503-509.

MYHRE HO. Reactive hyperaemia of the human lower limb. Measurement of postischaemic blood flow velocity in controls and in patients with lower limb atherosclerosis. Vasa 1975; : 145-149.

NAYMAN J. The use of the ult rasonic flow meter in peripheral vaseular disease. Aust NZ J Surg 1974: 44:157-167.

NEEDHAM T, YAO ST. Assessment of arterial disease of the leg by ull rasound and plethysmograplyy. In: Blood flow measurement. (Ed. ROBERTS Ci Scctor, London 1972: pp. 65-68.

NICOLAIDES AN, GORDON-SMTH IC. DAYANDAS J, EASTCOTT HHC. The value of Doppler blood velocity tracings in the detection of aortoiliac disease in patients with intermittent claudication. Surgery 1976;80:774-778.

NIMURA Y. MATSUOH. HAYASHIT, KITABATAKE A. MOCHIZUKIS.SAKAKIBARAH. KATO K. ABE H. Studies on arterial flow patterns-instantaneous velocity spectrums and their phasic changes-with directional ultrasonic Doppler technique. Br Heart J 1974: $36: 899-907$.

NIPPA JH, HOKANSON DE, LEE DR, SUMNER DS, STRANDNESS DE. Phase rotation for separating forward and reverse blood velocity signals. IEEE Trans Son Ultrason 1975 ; $S U-22: 340-346$.

O'DONNELL THF, PAUKER SG, CALLOW AD. KELLY JJ, MCBRIDE KJ.KORWINS. ThO relative value of carotid noninvasive testing as determined by receiver operator characteristic curves. Surgery 1980;87:9-17.

OROURKE ME. TAYLOR MG. Vascular impedance of the femoral bed. Circ Res 1966; $18: 126-139$.

PASCARELLI EF. BERTRAND ChA. Comparison of blood pressures in the arms and legs. New Engl J Med 1964: 270: 698-698.

PERSSON AV, GRIFFEY S. KORPRESKIM. Use of the non-invasive vascular laboratory as an adjuvant to clinical vascular surgery. In: Hemodynamique des membres - 1. (Ed. PUEL P. et al) G.E.P.E.S.C. Toulouse 1979; pp. 535-541.

PERONNEAU PA. BOURNAT JP. BUGNON A. BARBET A. XHAARD M. Theoretical and practical aspects of pulsed Doppler howmetry: real-time applicat ion to the measure of instantaneous velocity profiles in vit ro and in viw. In: Cardiovascular applications of ultrasound. (Ed. RENEMAN RS.) North Holland Publishing. Amsterdam 1974; Chap ter 6. pp. 66-84.

POLLAK EW. CHAVIS P. WOLFMAN EF. The effect of postural changes upon the ankle arterial perfusion pressure. Vasc Surg 1976;10:219-222.

RAAD VOOR GEZONDHEIDSRESEARCH TNO. Hart-en vaatuickten. Een bijdrage tot het onderzoeksbeleid. Staatsuitgeverij. "s-Gravenhage 1980.

RAINES JK. DARLING RC. BUTH J,BREWSTER DC. AUSTEN WG Vascular laboratory criteria for the management of peripheral vascular disease of the lower extremities. Surgery 1976:79:21-29.

RAINES I. Vascular laboratory evaluations using the pulse volume recorder. In Vascular surgery. (Ed. RUTHERFORD RB.) Saunders. Philadelphia 1977, Chapter 6, pp. 57-71. 
RAM MD. BHIMANIBK. Studies on velocily blood flow with the ultrasonic flow meter. Surg Gynecol Obstet 1971:133:815-820.

RENEMAN RS, CLARKEHF, SIMMONS N, SPENCER MP. In wivo comparison of electromagnetic and Doppler flowmeters: with spectal attention to the processing of the analogue Doppler flow signal. Cardiovasc Res 1973; 7:557-566.

RENEMAN RS. SPENCER MP. Difficulties in processing of an analogue Doppler flow signal; with special reference to zero-crossing meters and quantification. In: Cardiovascular applications of ultrasound. (Ed. RENEMAN RS.) North-Holland Publishing, Amsterdam 1974, Chapter 3, pp. 32-42.

RENEMAN RS. HOEKS A. Continuous wave and pulsed Doppler flowmeters - a general introduction. In: Echocardiology (Ed. BOM N.) Nijhofr, the Hague 1977; pp. 189-205.

RENEMAN RS, SPENCER MP. Local Doppler audio spectra in normal and stenosed carotid arteries in man. Ultrasound Med Biol 1979;5:1-11

RENEMAN RS. HOEKS A. SPENCER MP. Doppler ultrasound in the evaluation of the peripheral arterial circulation. Angiology $1979 ; 30: 526-538$.

RENEMAN RS. What measurements are necessary for adequate evaluation of the peripheral arterial circulation. Cardiovase Dis $1981 ; 8: 435-454$

RITTENHOUSE EA, MAIXNER W, BURR JW, BARNES RW. Directional arterial flow velocity: A sensilive index of changes in peripheral vascular resistance. Surgery 1976 $79: 350-355$.

RITTENHOUSE EA, STRANDNESS DE. Oscillatory flow patterns in patients with aortic valve disease. Am J Cardiol 1971; $28: 568-574$.

ROBERTS WR, SIGEL B. GIBSON RJ, WILLIAMS J, POPKY GL. EDELSTEIN AL, JUSTIN JR. Pulsed Doppler ultrasound detection of flow disturbances in arteriosclerosis. JCU 1976; 4:275-282.

ROBERTS C. Ultrasound in the assessment of vascular function. Med Prog Technol 1976 ; $4: 3-10$.

ROBICSEK F. The safety of lumbar aortography. Surgery $1978 ; 83: 489-490$.

RUISSEN CJ. Frequentie-multiplexing bij medisch onderzoek. Polytechn tijdschr 1980: $35: 372-373$.

RUTHERFORD RB. (Ed.) Vascular surgery. Saunders, Philadelphia 1977.

SAINZ A, ROBERTS VC, PINARDIG. Phase-locked loop techniques applied to ultrasonic Doppler signal processing. Ultrasonics, 1976; mav: 128-132.

SAINZ A, ROBERTS VC, PINARDI G. LINDENAUER SM. Blood flow velocity and acceleration measurement by doppler shift ultrasound. In: Non-invasive Clinical Measurement. (Eds. TAYLOR D, WHAMOND J.) Pitman Medical, Turnbridge Wells, 1977. Chapter 1, pp. 6-18.

SCHULTZ RD. HOKANSON DE. STRANDNESS DE. Pressureflow and stress-strain measu rements of normal and diseased aortoil hac segments. Surg Gynecol Obstet 1967; $124: 1267-1276$

SCHUTZ RM. Verlaufskontrolle der arteriellen Verschlusskrankheiten in der Praxis und prognostische Aspekte. MMW 1978; 120:37-42.

SCOT $\mathrm{N}$. Bio-impedance measurement. In: Non-invasive Clinical Measurement. (Eds. TAYLOR D. WHAMOND J.) Pitman Medical, Turmbridge Wells, 1977, Chapter 4, pp. 44-59.

SETHI GK, SCOTT SM. TAKAROT. Multi-plane angiography for more precise evaluation of aortollac disease. Surgery $1975 ; 78: 154-159$.

SIGGAARD-ANDERSEN J. ULRICH J, ENGELL HC, BONDE PETERSEN F. BlOOd pressure measurements of the lower limbs. Angiology 1972;23:350-357.

SKIDMORE R, BIRD DR, WOODCOCK JP, DAVIES PW, BAIRD RN.Transfer function analysis of common femoral artery Doppler waveforms. Br J Surg 1979:66:883.

SMITH RC. FERRINGTON $C$, RUCKLEY $C V$. Calf muscle technetium clearance and Doppler ankle pressure in patients with intermittent claudication. Vasa 1977;6:236243.

SPENCER MP, REID JM. Quantitation of carotid stenosis with continuous-wave (C-W) Doppler ultrasound. Stroke 1979;10:326-330. 
SPITTELL JA, WALLACE RB. Aneurysms, In: Allen-Barker-Hines ${ }^{2}$ Periphal wascular diseases. (Eds. JUERGENS JL, SPITTELL JA, FAIRBAIRN II JF, Saunders, Philladelphia, 1980; (5th ed), Chapter 14, pp. 415-437.

STAHLER Ch, STRANDNESS DE. Ankle blood pressure response to graded treadmill exercise. Angiology 1967; 18:237-241.

STRANDNESS DE, BELL JW. An evaluation of the hemodynamic response of the claudicating extremity to exercise. Surg Gynecol Obstet 1964;119:1237-1242.

STRANDNESS DE, MCCUTCHEON EP, RUSHMER RF. A pplication of a transcutaneous doppler flowmeter in evaluation of occlusive arterial disease. Surg Gynecol Obstet $1966 ; 122: 1039-1045$.

STRANDNESS DE, SCHULTZ RD, SUMNER DS, RUSHMER RF. Ultrasonic flow detection. A useful technic in the evaluation of peripheral vascular disease. Am J Surg 1967; 113 311-320.

STRANDNESS DE. Peripheral Arterial Disease. A pshysiological approach. Churchill, London 1969.

STRANDNESS DE, KENNEDY JW, JUDGETP, MCLEOD FD. Transcutaneous directional flow detection: A preliminary report. Am Heart J 1969; 78:65-74.

STRANDNESS DE. Evaluation of the patient for vascular surgery. Surg Clin North Am $1974 ; 54: 13-22$.

STRANDNESS DE, SUMNER DS. Hemodynamics for surgeons. Grune \& Stratton, New York 1975.

STRANDNESS DE. Exercise ankle pressure measurements in arterial disease. In: Noninvasive diagnostic techniques in vascular disease. (Ed. BERNSTEIN EF.) Mosby, Saint Louis, 1978; Chapter 28, pp. 315-321.

STRANDNESS DE. The use and abuse of the vascular laboratory. Surg Clin North Am $1979 ; 59: 707-717$.

SUMNER DS, STRANDNESS DE. The relationship between calf blood flow and ankle blood pressure in patients with intermittent claudication. Surgery $1969 ; 65: 763-771$.

SUMNER DS. Digital plethysmography. In: Vascular surgery (Ed. RUTHERFORD RB.) Saunders, Philadelphia, 1977; Chapter 7, pp. 73-78.

SUMNER DS. The hemodynamics and pathophysiology of arterial disease. In: Vascular surgery (Ed. RUTHERFORD RB.) Saunders, Philadelphia, 1977; Chapter 4, pp. 25-46.

SUMNER DS, STRANDNESS DE. Aortoiliac reconstruction in patients with combined iliac and superficial femoral arterial occlusion. Surgery 1978; $84: 348-355$.

TAKS ACIM. De femoro-popliteale bypass en samengestelde ingrepen. Thesis, Nijmegen 1978.

THULESIUS O. Beurteilung des Schweregrades arterieller Durchblutungsstörungen mit dem Doppler-Ultraschall-Gerät. In: Messmethoden bei arteriellen Durchblutungsstörungen. (Eds. BOLLINGER A. BRUNNER U.) Huber, Bern 1971: pp. 44-57.

THULESIUS O, GJÖRES JE. Use of Doppler shift detection for determining peripheral arterial blood pressure. Angiology 1971; $22: 594-603$.

ToNNESEN KH. Muscle blood flow during exercise in intermittent claudication. Validation of the 133 Xenon clearance technique: Clinical use by comparison to plethysmography and walking distance. Circulation $1968 ; 37: 402-410$.

UDOFF EJ, BARTH KH, HARRINGTON DP, KAUFMAN SL, WHITE RI. Hemodynamic significance of iliac artery stenosis: Pressure measurements during angiography. Radiology 1979;132:289-293.

VAN DE WATER JM, DMOCHOWSKI JR, DOVE GB, COUCH NP. Evaluation of an impedance flowmeter in arterial surgery. Surgery 1971; 70:954-961.

VAN DE WATER JM, MOUNT BE, ROETTINGER WF, TRUDELL LA. Noninvasive assessment of the peripheral vascular system. Arch Surg 1977;112:679-683.

VECCHIO TJ. Predictive value of a single diagnostic test in unsellected populations.

N Engl J Med 1966;274:1171-1173.

VOLLMAR J. Rekonstruktive Chirurgie der Arterien. Thieme, Stuttgart 1975.

VROONHOVEN TIMV van Sexual dysfunction after aorto-iliac surgery. Vasa $\| 977 ; 6$ : 226-229. 
WROONHOVEN TJMV van, MULLER H. Arteriosclerotische vernauwingen wan de distale aorta bij vrouwer vón de menopauze. Ned Tijdschr Geneeskd 1978; 122:321-325. WARD AS. MORRIS-JONES $\mathrm{W}$. The role of the profunda femoris in aorto-iliac surgery. BI J Surg 1978; 65: 308-312.

WARD AS. MARTIN TP. Some aspects of ultrasound in the diagnosis and assessment of aortoiliac disease. Am J Surg 1980; 140:260-265.

WATERS KJ. CHAMBERLAIN J, MCNEILL IF. The significance of Aortoiliac atherosclerosis as assessed by Doppler ultrasound. Am J Surg 1977, 134: 388-391.

WELLSS PNT. The possibility of harmful biological effects in ultrasonic diagnosis. In: Cardiovascular applications of ultrasound. (Ed. RENEMAN RS.) North-Holland Publishing. Amsterdam 1974a; Chapter 1, pp. 1-17.

WELLS PNT. Ultrasonic Doppler probes. In: Cardiovascular applications of ultrasound. (Ed. RENEMAN RS.) North-Holland Publishing, Amsterdam 1974b; Chapter 9, pp. 125-131.

WESOLOWSKI SA, MARTINEZ A, DOMINGO RT, FRIES CC, SCHAEFER HC, SAWYER PN. GILLIE E, MCMAHON ID. Indications for aortofemoral arterial reconstruction: A study of borderline risk patients. Surgery 1966;60:288-298.

WESTERHOF N, SIPKEMA P, BOS GC van den, ELZINGA G. Forward and backward waves in the arterial system. Cardiovasc Res 1972;6:648-656.

WOODCOCK JP, GOSLING RG, FITZGERALD DE. A new non-inwasive technique for assessment of superficial femoral artery obstruction. Br J Surg 1972a; 59: 226-23l.

WOODCOCK J, GOSLING R, KING D, NEWMAN D. Physical aspects of blood-velocity measurement by Dopplershifted ultrasound. In: Blood flow measurement. (Ed. ROBERTS C.) Sector, London $1972 \mathrm{~b}$; pp. 19-23.

WOODCOCK JP. Analysis of doppler-shift signals in ultrasonic flowmeters. In: Noninvasive Clinical Measurement. (Eds TAYLOR D. WHAMOND J.) Pitman Medical, Turnbridge Wells 1977; Chapter 6, pp. 82-97.

WYLIE EI, GOLDMAN L. The role of aortography in the determination of operability in arteriosclerosis of the lower extremities. Ann Surg 1958;148:325-342.

YAO ST, HOBBS JT, IRVINE WT. Pulse examination by an ultrasonic method. Br Med J 1968; $4: 555-557$.

YAO ST. Haemodynamic studies in peripheral arterial disease. Br J Surg 1970; 57: 761766.

YAO ST, NEEDHAM TN, ASHTON JP. Transcutaneous measurement of blood now by ultrasound. Bio-Medical Engineering 1970; 230-233.

YAO JST. New techniques in objective arterial evaluation. Arch Surg 1973; 106:600-604.

YAO JST, BERGAN JJ. Application of ultrasound to arierial and venous diagnosis. Surg Clin North Am 1974; $54: 23-38$.

YOUNG DF, CHOLVIN NR, KIRKEEIDE RL, ROTH AC. Hemodynamics of arterial stenoses at elevated flow rates. Circ Res 1977; 41:99-107. 
Peter J.E.H.M. Kitslaar was born on the 6th of June 1943 in Roermond. After completing grammar school in that same town he studied at the Medical Faculty of the University of Nijmegen, the Netherlands, and graduated in 1969 .

After a one-year training in surgery (St. Hippolytus Hospital, Delft) and courses in tropical medicine, he worked for three years as a Medical Officer in the rural Sumve District Hospital in Tanzania, East Africa. In February 1974 he started his formal surgical training in the St. Antonius Hospital, Utrecht (Heads: first Dr. A.L.E.M.S. Schaepkens van Riempst, later on Dr. H.J. Gelissen). In February 1980 he was registered as a surgeon and continued working as such in the St. Antonius Hospital until in January 1982 he was appointed surgeon in the Deaconesses' Hospital in Groningen, the Netherlands. 